\title{
Dynamics of soft interfaces in droplet-based microfluidics
}

\section{Dissertation}

zur Erlangung des mathematisch-naturwissenschaftlichen

Doktorgrades

"Doctor rerum naturalium"

der Georg-August-Universität Göttingen im Promotionsprogramm ProPhys

der Georg-August University School of Science (GAUSS)

vorgelegt von

Quentin Brosseau

aus Saint-Nazaire, Frankreich

Göttingen, 2014 


\section{Betreuungsausschuss:}

Prof. Dr. Jörg Enderlein, Drittes Physikalisches Institut, Universität Göttingen

Prof. Dr. Jean-Christophe Baret, Soft Micro Systems, Centre de Recherche Paul Pascal, Université de Bordeaux, Pessac (Fr.)

Tröpfchen, Membranen und Grenzflächen, Max-Planck-Institut für Dynamik und Selbstorganisation, Göttingen

Prof. Dr. Stephan Herminghaus, Dynamik komplexer Fluide, Max-Planck-Institut für Dynamik und Selbstorganisation, Göttingen

\section{Mitglieder der Prüfungskommission}

Referent : Prof. Dr. Jörg Enderlein, Drittes Physikalisches Institut, Georg-August-Universität Göttingen

Koreferent : Prof. Dr. Jean-Christophe Baret, Soft Micro Systems, Centre de Recherche Paul Pascal, Université de Bordeaux, Pessac (Fr.)

Tröpfchen, Membranen und Grenzflächen, Max-Planck-Institut für Dynamik und Selbstorganisation, Göttingen

\section{Weitere Mitglieder der Prüfungskommission}

Prof. Dr. Stephan Herminghaus, Dynamik komplexer Fluide, Max-Planck-Institut für Dynamik und Selbstorganisation, Göttingen

Prof. Dr. Sarah Köster, Institut für Röntgenphysik / Courant Research Centre, Georg-August-Universität Göttingen

Prof. Dr. Marcus Müller, Institut für Theoretische Physik, Georg-August-Universität Göttingen

Prof. Dr. Philipp Vana, Institut für Physikalische Chemie, Georg-August-Universität Göttingen

Tag der mündlichen Prüfung: 14. April 2014 um 14h00. 


\section{Abstract}

This doctoral thesis presents the study of different dynamic processes occurring at the droplet interface, and the methods implemented for their study. The objective of this work is to unravel what are the important properties that have an impact on the mechanical behavior of the interfacial layers. We use the hydrodynamically forced droplet deformation in a microfluidic channel to probe the mechanical properties of the interface. This method is applied to three different cases.

First we follow the evolution of an interface deformation with time to study the dynamics of surfactant adsorption to the interface. In this study we formulate the calibration of the droplet deformation as a function of the droplet size and surface tension. We apply this technique to the case of a perfluorosurfactant of interest in industrial and research applications. We show the possibility to measure the dynamic surface tension for time scales down to 10 milliseconds and extract kinetic properties of the molecule. We compare the dynamics obtained by the classical pendant drop method and in microfluidics. We show that the regime of adsorption is diffusion-limited in pendant drop and follow the scaling of a transfer-limited regime in microfluidics. The difference is explained by the flow profile that induce convective transport in microfluidics.

Then we study the droplet deformation under different confinement conditions in microfluidics. The droplet deformation is compared to a two dimensional numerical simulation and to a three dimensional model of deformation of a spheroidal droplet. In both cases a qualitative agreement is found with the model but noticeable deviations occur experimentally. The deviation from two dimensional model is visible through the decrease of the influence of the viscous stress with the channel height, due to contributions of out-of-plane deformations that are not included in the model. The deviation from the three dimensional model comes from the confinement of the droplet which modify the droplet shape from a spheroid. This study shows the difficulty of describing interfacial viscous stresses in confined geometries which are to large for being considered as two dimensional, but where interactions with the channel walls are non negligible. We discuss also the inertial relaxation of the droplet at Reynolds number $R e \sim 10$ featuring oscillations of the droplet interface. We show that these oscillations can be described using a hydrodynamic analogy of a damped Hookian spring taking into 
account the surface tension as a spring constant, and a damping related to the fluids viscosity. The analysis of the frequency is valid for pure interfaces as well as for surfactant covered interfaces, leading to an additional way to access the surface tension via the frequency of the interface deformation. The viscous relaxation here as well is found to be influenced by the channel walls.

Finally we apply the method of the microfluidic tensiometry to study the kinetics of a polymerization reaction occurring at the droplet interface. The effect of the reagent concentration on the reaction time is studied as well as the effect of the presence of surfactant molecules at the interface. The preliminary results of this study show that the deformation of a complex interface can no more be described by the surface tension only. The description of the mechanical properties of the interface necessitate to take into account the emergence of the interfacial viscoelasticity upon polymerization. This conclusion introduces the possibilities that microfluidics may offer to address the characterization of the mechanical properties of complex interfaces such as colloid laden interfaces, or membranes. 


\section{Kurzzusammenfassung}

Diese Doktorarbeit untersucht die verschiedenen dynamischen Prozesse, welche sich an der Tropfenoberfläche abspielen, und der Methoden, die für deren Untersuchung verwendet wurden. Das Ziel dieser Arbeit ist es, die entscheidenden Eigenschaften, die einen Einfluss auf das mechanische Verhalten der Grenzfläche haben, zu identifizieren. Wir verwenden die hydrodynamisch erzwungene Deformation eines Tropfens in einem Mikrokanal, um die mechanischen Eigenschaften der Oberfläche zu untersuchen. Diese Methode wird auf drei verschiedene Fälle angewendet.

Als erstes verfolgen wir die zeitliche Entwicklung einer Grenzflächenverformung, um die Dynamik der Tensidadsorption an einer Oberfläche zu untersuchen. Dabei kalibrieren wir die Tropfenverformung als Funktion von Tropfengröße und Oberflächenspannung. Diese Technik wird auf den Fall eines perfluorierten Tensids, welches von industriellem und wissenschaftlichem Interesse ist, angewendet. Wir zeigen die Möglichkeit von Messungen der dynamischen Oberflächenspannung auf Zeitskalen von zehn Millisekunden und gewinnen daraus kinetische Eigenschaften der Moleküle. Wir vergleichen die Dynamik, welche mit der klassischen Pendant-Drop-Methode gemessen werden kann mit denen der Mikrofluidik. Es zeigt sich, dass die Adsorption für den Pendant Drop von der Diffusion begrenzt wird, während in der Mikrofluidik die Anbindung an die Oberfläche der langsamere Prozess ist. Der Unterschied entsteht durch das Flussprofil in der Mikrofluidik, welches konvektiven Transport induziert.

Danach untersuchen wir die Verformung unter verschiedenen räumlichen Beschränkungen im mikrofluidischen Kanal. Die Tropfenverformung wird mit einer zweidimensionalen numerischen Simulationen und mit einem dreidimensionalen Modell eines Rotationsellipsoids verglichen. In beiden Fällen wird eine qualitative Übereinstimmung festgestellt, jedoch existieren auch spürbare Abweichungen vom Experiment. Die Abweichungen vom zweidimensionalen Modell ist erklärbar mit dem sinkenden Einfluss der viskosen Spannungen mit der Kanalhöhe, hervorgerufen durch Beiträge von Deformationen außerhalb der Beobachtungsebene, welche von dem Modell nicht wiedergegeben werden. Die Abweichungen vom dreidimensionalen Modell kommen von den räumlichen Beschränkungen, welche die Tropfenform von einem Rotationsellipsoid abweichend verformt. Die Untersuchung zeigt die Schwie- 
rigkeiten bei der Beschreibung von viskosen Kräfte für Abmessungen, die zu groß sind um als zweidimensional betrachtet zu werden, aber wo die Wechselwirkungen mit den Kanalwänden nicht vernachlässigbar sind. Wir diskutieren ebenfalls den Fall der trägen Relaxation des Tropfens bei Reynoldszahlen von $R e \sim 10$, für welchen Oszillationen der Tropfenoberfläche beobachtet werden. Wir zeigen, dass die Oszillationen als hydrodynamische Analogie zu einer hookeschen Feder beschrieben werden können, wobei die Oberflächenspannung als Federkonstante fungiert und die Dämpfung durch die Viskosität der Flüssigkeit bestimmt wird. Die Methode liefert korrekte Ergebnisse sowohl für reine Grenzflächen als auch für Grenzflächen mit Tensiden, was zu einer zusätzliche Möglichkeit führt, die Oberflächenspannung aus der Frequenz der Verformungen zu bestimmen. Die viskose Relaxation wurde auch hierbei von den Kanalwänden beeinflusst.

Als letztes wenden wir die Methode der mikrofluidischen Tensiometrie auf die Kinetik einer Polymerisationsreaktion auf der Tropfenoberfläche an. Der Einfluss der Reagenzkonzentration auf die Reaktionszeit wird untersucht, ebenso wie der Effekt der Gegenwart von Tensidmolekülen. Erste Ergebnisse dieser Untersuchung zeigen, dass die Deformation einer komplexen Grenzfläche nicht mehr allein durch die Oberflächenspannung beschrieben werden kann. Vielmehr muss die Beschreibung der mechanischen Eigenschaften der Grenzfläche notwendigerweise die Entstehung der Viskoelastizität an der Oberfläche mit in Betracht ziehen. Diese Erkenntnis eröffnet neue Möglichkeiten, mit Hilfe von Mikrofluidik die mechanischen Eigenschaften von komplexen Grenzflächen, wie zum Beispiel kolloidbesetzte Grenzflächen oder Membranen, zu charakterisieren. 


\section{Contents}

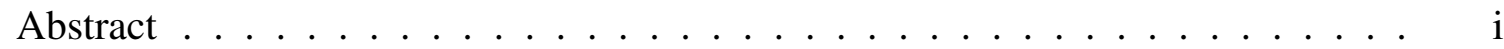

Contents . . . . . . . . . . . . . . . . . . . . viii

List of Figures . . . . . . . . . . . . . . . . . . xi

List of Tables . . . . . . . . . . . . . . . . . . . . xiii

1 Introduction 1

1.1 Soft interfaces . . . . . . . . . . . . . . . . . . . . 1

1.2 Droplet-based microfluidics . . . . . . . . . . . . . . . . 4

1.3 Thesis outline $\ldots \ldots \ldots \ldots \ldots \ldots \ldots$

\begin{tabular}{|lll}
2 & Surface tension and capillary forces & 7
\end{tabular}

$2.1 \quad$ Origins of the surface tension . . . . . . . . . . . . . . . 7

2.2 Capillary forces $\ldots \ldots \ldots \ldots \ldots$. . . . . . . . . . . . . . . 9

$2.2 .1 \quad$ Laplace pressure . . . . . . . . . . . . . . . . . . 9 9

2.2 .2 Contact line forces . . . . . . . . . . . . . . . . . . 11

2.3 Methods of measurement . . . . . . . . . . . . . . . . . . . . . . 12

$2.3 .1 \quad$ Pendant drop tensiometry . . . . . . . . . . . . . 13

2.3 .2 Whilhelmy plate tensiometry . . . . . . . . . . . . . 15

\begin{tabular}{|lll}
3 & Adsorption of surfactant to the interface & 17
\end{tabular}

3.1 Surfactant molecules . . . . . . . . . . . . . . . . . . . . . 17

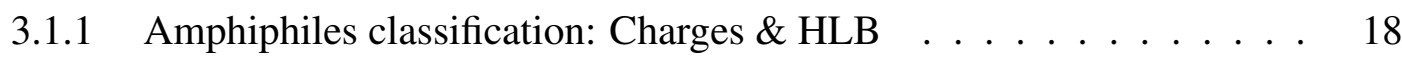

3.1 .2 CMC \& Micelization processes . . . . . . . . . . . . . . . . 19

3.2 Kinetics of surfactant adsorption . . . . . . . . . . . . . . . . . 21

3.3 Equilibrium equation of state $\ldots \ldots \ldots \ldots . \ldots \ldots 22$

3.4 Adsorption isotherms $\ldots \ldots \ldots \ldots$. . . . . . . . . . . . . . . . 24

3.4 .1 Langmuir Isotherm . . . . . . . . . . . . . . . . . . . 24 
$3.4 .2 \quad$ Henry Isotherm $\ldots \ldots \ldots \ldots$

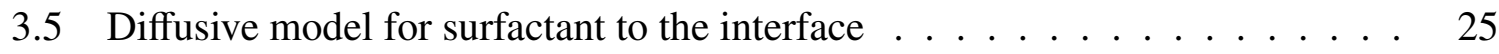

3.5.1 Solution of the diffusion equation in the asymptotic cases. . . . . . . 27

3.6 Further transport models $\ldots \ldots \ldots \ldots \ldots \ldots \ldots$

$3.6 .1 \quad$ Convective transport $\ldots \ldots \ldots \ldots \ldots \ldots \ldots$

3.6 .2 Micellar adsorption $\ldots \ldots \ldots \ldots \ldots \ldots \ldots$

$\begin{array}{|lll|}4 & \text { On-chip emulsification and hydrodynamics in microfluidic environment } & 31\end{array}$

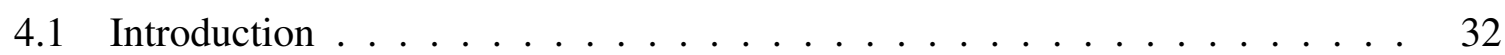

4.2 Principles of on-chip emulsification $\ldots \ldots \ldots \ldots \ldots \ldots \ldots$

$4.2 .1 \quad$ Emulsion aging and methods of stabilization $\ldots \ldots \ldots \ldots$

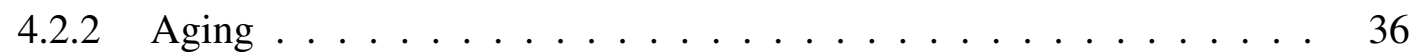

4.2 .3 Stabilization $\ldots \ldots \ldots \ldots \ldots \ldots \ldots \ldots \ldots \ldots$

4.3 Hydrodynamics in confined flow . . . . . . . . . . . . . . 41

4.3 .1 The Navier-Stokes equations . . . . . . . . . . . . . . . . . . 41

4.3 .2 Reynolds number . . . . . . . . . . . . . . . . . . . 42

4.3 .3 Interfacial limit conditions $\ldots \ldots \ldots \ldots \ldots$. . . . . . . . 44

\begin{tabular}{|lll}
5 & Experimental methods & 47
\end{tabular}

$5.1 \quad$ Surface tension measurements: Pendant drop method $\ldots \ldots \ldots \ldots$. . . . 47

5.2 Dynamic light scattering $\ldots \ldots \ldots \ldots \ldots \ldots \ldots \ldots$

5.3 Microfabrication . . . . . . . . . . . . . . . . . . 49

$5.3 .1 \quad$ Clean Room techniques $\ldots \ldots \ldots$

5.3 .2 Fabrication of the NOA chips $\ldots \ldots \ldots \ldots \ldots$. . . . . . . 51

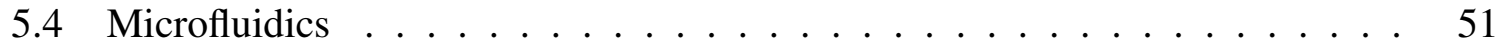

$5.4 .1 \quad$ Fluid control . . . . . . . . . . . . . . . . . . . . . . . 51

5.4 .2 High-Speed Microscopy $\ldots \ldots \ldots \ldots \ldots$. . . . . . . . . 52

5.5 Image processing and data extraction $\ldots \ldots \ldots \ldots \ldots \ldots \ldots$

5.5 .1 Comments on the method $\ldots \ldots \ldots \ldots$. . . . . . . . . . 54

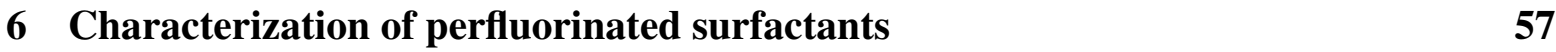

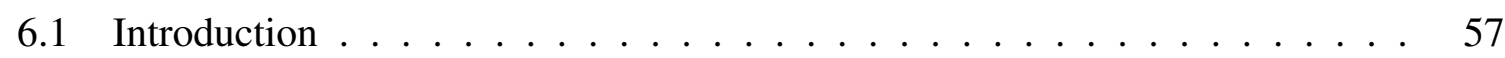

6.2 Molecular structures $\ldots \ldots \ldots \ldots$

6.3 Method . . . . . . . . . . . . . . . . . . . 60

6.4 Diffusion-limited adsorption and scalings $\ldots \ldots \ldots \ldots \ldots \ldots$

$6.4 .1 \quad$ Block copolymer PEG-PFPE $\ldots \ldots \ldots \ldots$. . . . . . . . . . . 62 
6.5 Charged surfactant Krytox-H and Krytox-NH $\mathrm{NH}_{4} \ldots \ldots \ldots$

6.5 .1 General form of the dynamics . . . . . . . . . . . . 67

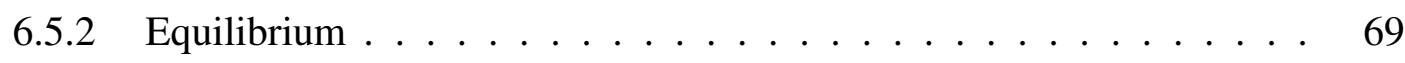

6.5 .3 Kinetics of adsorption. . . . . . . . . . . . . . . . 74

6.6 Dynamic Light Scattering: Micellarization processes . . . . . . . . . . . 81

6.6 .1 Macromolecule PEG-PFPE . . . . . . . . . . . . 81

6.6 .2 PFPE chains . . . . . . . . . . . . . . . . . 81

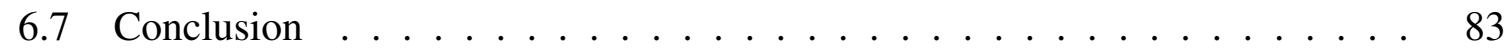

7 Microfluidic Dynamic Interfacial Tensiometry: $\mu \mathrm{DIT} \quad \mathbf{8 5}$

7.1 Introduction . . . . . . . . . . . . . . . . . . . . 85

7.2 Microfluidic geometry $\ldots \ldots \ldots \ldots \ldots$

7.3 Droplet deformation at a microfluidic expansion . . . . . . . . . . . . . 91

7.4 Dynamics of surfactant adsorption: Microfluidics . . . . . . . . . . . . . . . 95

7.4.1 Droplet deformation in presence of surfactants . . . . . . . . . . 95

7.4 .2 Dynamic microfluidic tensiometry . . . . . . . . . . . . . . . . . . . . . 97

7.5 Discussion . . . . . . . . . . . . . . . . . . . . 102

7.6 Conclusions . . . . . . . . . . . . . . . . . . . . 103

8 Deformation of droplet interface in microfluidics environments $\quad 105$

8.1 Introduction . . . . . . . . . . . . . . . . . . . . . 105

8.2 Droplet deformation in a planar sudden expansion . . . . . . . . . . . . . 106

$8.2 .1 \quad$ Experimental method . . . . . . . . . . . . . . . 107

8.2 .2 Numerical two-dimensional model . . . . . . . . . . . . . . . . 108

8.2 .3 Results . . . . . . . . . . . . . . . . . . . . . . . . 109

8.2 .4 Surfactant covered droplets . . . . . . . . . . . . . . . . . . 114

8.2 .5 Discussion . . . . . . . . . . . . . . . 115

8.3 Deformation of spheroidal droplets in elongational flow profile . . . . . . . . 118

8.4 Droplet relaxation in inertial regime . . . . . . . . . . . . . . . . . 123

8.4 .1 Model . . . . . . . . . . . . . . . . 123

8.5 Experimental results $\ldots \ldots \ldots \ldots \ldots \ldots \ldots$

$8.5 .1 \quad$ Dissipative component of the oscillations . . . . . . . . . . . . 125

8.5 .2 Droplet oscillations . . . . . . . . . . . . . . . . . . 127

8.5 .3 Oscillations in presence of surfactant . . . . . . . . . . . . . . 129

8.6 Conclusion . . . . . . . . . . . . . . . . . . . . 132 
9 Monitoring polymerization at the droplet interface using microfluidics 135

9.1 Introduction . . . . . . . . . . . . . . . . . . 135

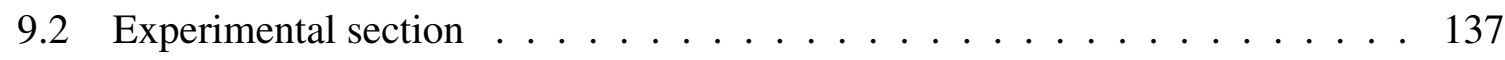

$9.2 .1 \quad$ Geometry . . . . . . . . . . . . . . . . . . . 137

9.2 .2 Polymerization reaction and chemicals $\ldots \ldots \ldots . \ldots 138$

9.3 Dynamics of interfacial polymerization monitored on-chip . . . . . . . . . . 139

9.3 .1 Profile of deformation of the interface . . . . . . . . . . . . . . . 139

9.3.2 Dynamics of the droplet maximum deformation . . . . . . . . . . . . 141

9.4 Conclusion . . . . . . . . . . . . . . . . . . . 146

10 Summary and Outlook 149

10.1 Summary . . . . . . . . . . . . . . . . . . . . . . . . 149

10.2 Outlook . . . . . . . . . . . . . . . . . . 152

A Measurement of PDMS channel deformation under flow conditions. 155

A.1 Droplet area change . . . . . . . . . . . . . . . . . . . 155

A.2 Confocal Fluorescence microscopy . . . . . . . . . . . . . . . . . . . 157

\begin{tabular}{ll}
\hline Appendices & 154
\end{tabular}

\begin{tabular}{ll}
\hline Appendices & 154
\end{tabular}

\begin{tabular}{ll}
\hline References & 161
\end{tabular} 


\section{List of Figures}

1.1 Examples of simple and complex interfaces . . . . . . . . . . . . 3

1.2 Micrometric emulsion . . . . . . . . . . . . . . . . . . . . . . 4

$2.1 \quad$ Why spherical drops in space $\quad \ldots \ldots \ldots$. . . . . . . . . . . 9

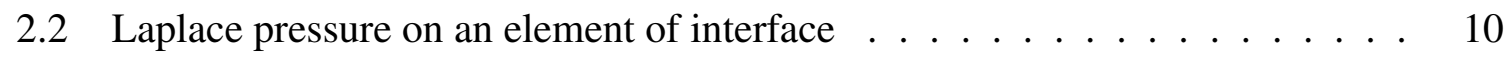

2.3 The Young-Laplace equation and the wetting of surfaces . . . . . . . . . . 11

2.4 Review of classical surface tension measurement methods and their characteristic time scales of application . . . . . . . . . . . . . . . . 13

2.5 The pendant drop tensiometry $\ldots \ldots \ldots \ldots \ldots$. . . . . . . . . . . . . . . . . . . . . . . . .

2.6 The Wilhelmy plate tensiometry . . . . . . . . . . . . . . . 16

$3.1 \quad$ Examples of surfactant molecules and micelles $\ldots \ldots \ldots$. . . . . . . 18

3.2 Phase diagram of the micelles structure . . . . . . . . . . . . 20

3.3 Adsorption of surfactant to the interface . . . . . . . . . . . . . 22

3.4 Model for the kinetics of surfactant adsorption . . . . . . . . . . . . . 26

3.5 Model of adsorption of micelles to the interface . . . . . . . . . . . . . 29

4.1 Emulsification methods in microfluidics . . . . . . . . . . . . . . 34

4.2 Controlled emulsification with external fields . . . . . . . . . . . . 36

4.3 Techniques of on-chip multiple emulsification . . . . . . . . . . . . . . 37

4.4 Emulsion aging and stabilization $\ldots \ldots \ldots \ldots$

4.5 Hagen-Poiseuille flow in channel with rectangular cross-section . . . . . . . 43

4.6 Flow and resulting viscous stresses at the interface $\ldots \ldots \ldots$. . . . . . . 45

$5.1 \quad$ Setup used for pendant drop method measurements . . . . . . . . . . . . 48

5.2 Setup used for Dynamic Light Scattering measurements . . . . . . . . . . . 48

5.3 Protocol of NOA chip fabrication . . . . . . . . . . . . . . . . 50 
5.4 Setup used for on-chip droplet deformation measurements . . . . . . . . 52

5.5 Image processing diagram $\ldots \ldots \ldots \ldots \ldots$

$6.1 \quad$ Changes in the partition properties of a dye in presence of surfactant . . . . 58

6.2 Chemical structure of the molecules used in our study . . . . . . . . . . . 60

6.3 Kinetics of adsorption of the PEG-PFPE molecules in pendant drop method . 65

6.4 Early kinetic of surface tension for PEG-PFPE molecules . . . . . . . . . 66

6.5 Dynamics of surface tension for Krytox-H molecules . . . . . . . . . . . . 68

6.6 Dynamics surface tensions for Krytox- $\mathrm{NH}_{4}$ molecules . . . . . . . . . . . . . 69

6.7 Equilibriums of the adsorption of PFPE molecules at oil / water interfaces . . 70

6.8 Study of the interfacial coverage of the air / water interface as a function of electrolytes concentration in the water phase . . . . . . . . . 73

6.9 Characteristic times of adsorption of Krytox-H molecules to the interface . . 75

6.10 Example of dimers stabilized by H-bonds . . . . . . . . . . . . . 76

6.11 Characteristic times of adsorption of Krytox- $\mathrm{NH}_{4}$ molecules to the interface . 79

6.12 DLS measurement applied to PEG-PFPE surfactant . . . . . . . . . . . . 82

7.1 Microfluidics geometry of the droplet deformation study $\ldots . . . . . . .88$

$7.2 \quad$ Droplet deformation profile in a microfluidic sudden planar expansion . . . . 90

7.3 Droplet deformation in function of the disperse phase viscosity . . . . . . . . 92

7.4 Dependence of the droplet maximum deformation on the Capillary number and on the droplet size . . . . . . . . . . . . . . . . . 93

7.5 Calibration of the droplet maximum deformation on the surface tension and the droplet size . . . . . . . . . . . . . . . . . . . . . 94

$7.6 \quad$ Dynamics measurement of the droplet speed and size parameters . . . . . . 95

7.7 Dynamics of surface tension measured on-chip . . . . . . . . . . . . 100

7.8 Example of a on-chip measurement of small surface tensions . . . . . . . . 101

8.1 Droplet maximum deformation $\delta_{\max }$ in function of the droplet confinement . . 110

8.2 Comparison of the droplet deformation profile obtained numerically and experimentally . . . . . . . . . . . . . . . . . . . . 111

8.3 Numerical simulations of the droplet maximum deformation in planar sudden expansion . . . . . . . . . . . . . . . . . . 112

$8.4 \quad$ Dependence of the Capillary number exponent on the channel height . . . . . 114

8.5 Deformation of a surfactant-laden interface at a planar sudden expansion . . . 116

$8.6 \quad$ Simulated droplet contour at the droplet maximum deformation . . . . . . . . 117 
8.7 Elongational flow profiles for a droplet entering the microfluidic constriction. 121

8.8 Taylor plots for three different channel heights . . . . . . . . . . . . . . . . 122

8.9 Analogy between the inertial relaxation of the droplet interface and a hookian

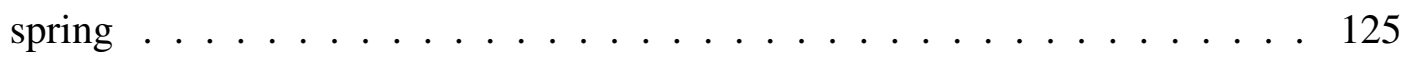

8.10 Comparison between the viscous relaxation time, and the droplet relaxation time 126

8.11 Dependence of the extracted frequency on the droplet size $R$, surface tension $\gamma$ and speed $U \ldots \ldots \ldots \ldots \ldots$. . . . . . . . . . . . . . . . . . . . . . . . 127

8.12 Droplet oscillation $\omega$ compared to the free relaxation frequency $\omega_{0} \ldots \ldots$

8.13 Oscillations of a surfactant-laden interface . . . . . . . . . . . . . . . . 131

$9.1 \quad$ Example of a complex interfaces designed for the purpose of target delivery - 136

9.2 Microfluidic geometry for the on-chip monitoring of polymerization reaction 138

9.3 Polymerization reaction between TDI and HMDA . . . . . . . . . . . . . . 139

9.4 Profiles of the droplet deformation upon polymerization . . . . . . . . . . . 140

9.5 Dependence of the reaction kinetics on the HMDA concentration . . . . . . . 142

9.6 Dependence of the reaction kinetics on the TDI concentration. . . . . . . . . 143

9.7 Dependence of the reaction kinetics in presence of surfactant . . . . . . . . . 145

10.1 One of the main achievement of this Ph.D. thesis f . . . . . . . . . . 150

A.1 Estimation of the PDMS channel deformation through the droplet change of size 156

A.2 Adsorption and Emission spectra for the Resorufin dye . . . . . . . . . . 158

A.3 Setup for confocal fluorescence microscopy measurement of the channel crosssection . . . . . . . . . . . . . . . . . . . . . 158

A.4 Result of Confocal Fluorescence Microscopy applied the the measurement of a PDMS microfluidic channel . . . . . . . . . . . . . . . . . . . . . 160 



\section{List of Tables}

2.1 Air / liquid surface tensions of a selection of liquids $\ldots \ldots \ldots$

6.1 Table of the kinetics parameters of the PEG-PFPE surfactant obtained in Pendant drop method . . . . . . . . . . . . . . . . 63

6.2 Equilibrium parameters of both molecules Krytox-H and Krytox- $\mathrm{NH}_{4}$ at the air / water interface . . . . . . . . . . . . . . . 71

6.3 Kinetics properties of molecules Krytox-H and Krytox- $\mathrm{NH}_{4}$ measured with pendant drop method . . . . . . . . . . . . . . 78

$7.1 \quad$ Kinetic parameters of the PEG-PFPE surfactant obtained on chip . . . . . . . 97

8.1 Surface tensions and densities of various water/ethanol mixtures . . . . . . . 108

$8.2 \quad$ Surface tension values extracted from the Taylor plots . . . . . . . . . . . . . 122 

à Dominique Marcandella, esprit curieux et ouvert $(14 / 12 / 1924-28 / 12 / 2012)$ 


\section{1 \\ Introduction}

\subsection{Soft interfaces}

Interfaces are ubiquitous in our everyday life and are an essential feature of complex fluids, living systems and technological systems. From a basic perspective, an interface is the boundary between two media having different physical or chemical properties. The mathematical definition of the interface faces the classical problem of discontinuity of physical quantities. This discontinuity can be formalized differently depending on the lenghtscale considered. Considering the interface as a two dimensional system to which excess quantities are attached is the basis of Gibbs approach[1]. Here the interface is seen as a two dimensional area, with an infinitesimal thickness, that precisely separates two phases in space. The thermodynamic or mechanical properties of the interface is then described by a set of parameters that are attached to the interface accounting for the discontinuities across the interface. This type of description is convenient for instance to describe the optical effect of light refraction that is depicted on figure 1.1. where the discontinuous change in index of refraction between the water phase and the air, bends the optical path of the light. As long as the lengthscale of the physical phenomenon (e.g. wavelength as the diffraction limit for an electromagnetic wave) is decoupled from the lengthscale of the fluctuations of the interface (typically the width of the transition zone), the two dimensional description will hold. 
The phenomenon of critical opalescence close to critical point is the most striking example of the breakdown of this approach in simple fluids: close to the critical points, the width of the interface becomes macroscopic affecting refraction at the interface. Considering lengthscales is therefore crucial. Under standard experimental conditions, the typical lengthscale of fluctuations of the interface is of order of the molecular scale. When considered at this scale, the interfaces are better represented by a transition region where properties of molecular concentrations and of the chemical potential vary continuously. This representation known as the Guggenheim representation[1] is more suitable to consider an interface as a complex environment, with a third dimension and also to account for the presence of inhomogeneities. In this framework, a large panel of object that range from the pure interface, to complex systems such as biological membranes (which are typically few molecular length thick) can be described.

Of course such a description can only be made at the expense of a detailed understanding of interactions, energies and specific physical-chemical features which are experimentally hardly accessible under dynamic conditions. Studies of surfactant exactly pinpoint this challenge. Surfactants are amphiphilic molecules therefore having affinities for interfaces. They are used in foams, (dispersion of a gas in a liquid), or as emulsions (dispersion of a liquid in an other liquid), as dispersant for solid particles and have a wide range of functions. They can be found in common food, cosmetics, drug delivery systems. In general, they affect the properties of the interface as a single monolayer organized on the two dimensional plane of the interface (the Gibbs approach), for example the mechanical properties of the interface. It is obvious that molecular properties have to be considered to understand the physical properties of such soft interfaces.

The general framework of this thesis is to unravel what are the important molecular properties that have an impact on the mechanical behavior of the interfacial layers. We focus on the study of a specific set of surfactant molecules that we characterize with standard methods. We show that microfluidic systems are versatile and efficient tools to analyze the dynamics of these soft interface, providing measurements of kinetics of adsorption desorption of surfactant, i.e. giving details on their behavior at the molecular level. Finally, the tools presented in the first part have a potential direct application for the monitoring of interfacial polymerization kinetics, another demonstration of the link that can be made between the macroscopic control of interfaces in microfluidics and specific details of chemical processes at the molecular level. 

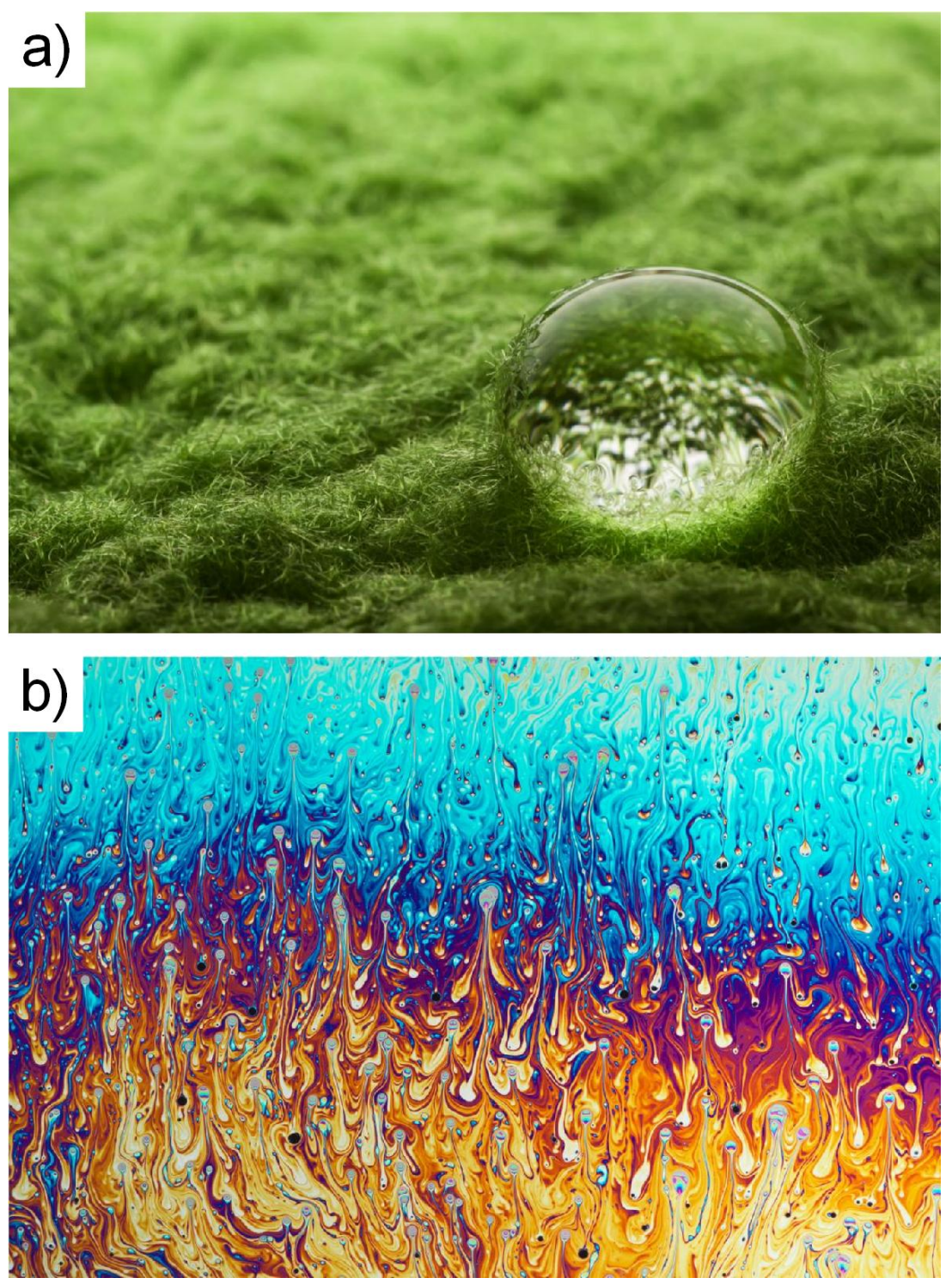

Figure 1.1 (a) The optical refraction of light passing through an air / water interface induced by the sudden change of the optical index, makes this droplet appears as a micro lens. (b) Closeup micrograph of a vertical soap film. The iridescences are linked to the interferences of the incoming light with the light reflected on the walls of the film. Therefore the color gradient are linked to the relative thickness of the film. This film is made stable by the presence of surfactant, and inhomogeneities in its distribution are visible from the swirl patterns. (C)Shawn Knol. 


\subsection{Droplet-based microfluidics}

Soft interfaces are easily produced using compartmentalization techniques and two phases flows in microfluidics [2]. The use of droplet as microreactors have been pushed by biochemical applications over the last 10 years and by on-chip emulsification techniques[3]. New concepts have emerged in this branch like the down-scaling of biological assays down to the size of micrometric droplets and droplet libraries where panels of thousand of droplets is used to perform drug screening. Microfluidic techniques constitute an ensemble that have a large

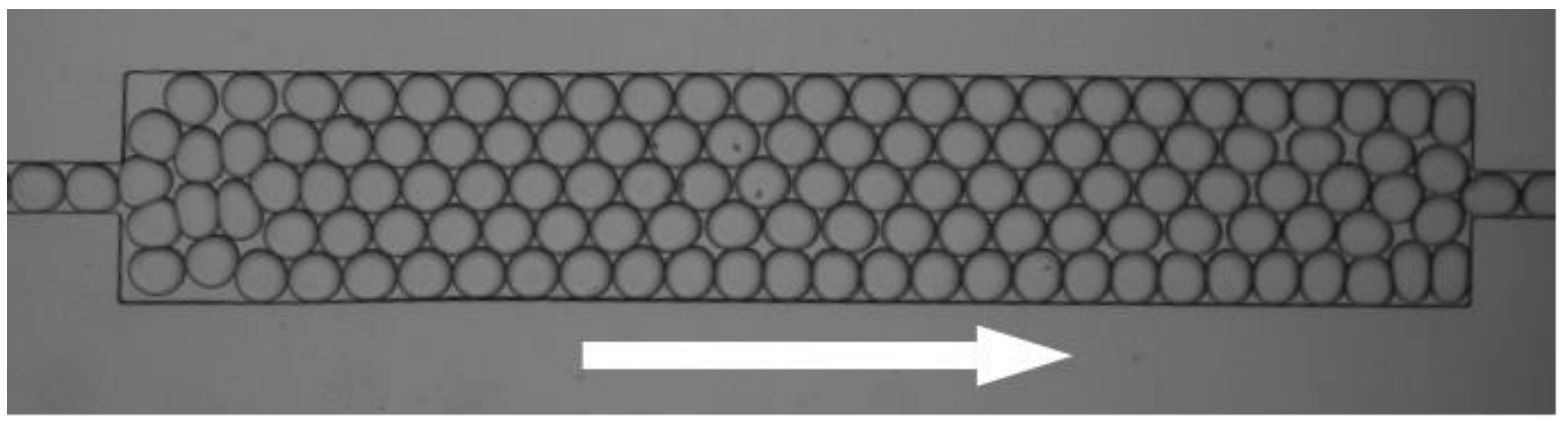

Figure 1.2 Micrometric emulsion flowing through a microfluidics channel. The flow direction is given by the arrow and the typical droplet size is $100 \mu \mathrm{m}$. The strong presence of interfaces is visible here and the stability of such emulsion is ensured by the presence of an adsorbed surfactant layer at the droplets interfaces.

potential for the study of interfaces at small scales, with enhanced control possibilities [4]. The area to volume ratio scales as $L^{-1}$, where $L$ is a typical length. Therefore the interfacial effects dominate at small scale, where it becomes possible to study the interfaces without the contribution of the bulk, see figure 1.2 . Finally, the control and the characterization of the interfaces for the application in microfluidics is motivated by the need for the good understanding of processes such as the emulsification and foaming. The study of interfaces stabilization is then at the core of this problematic. In this work the droplet deformation in microfluidics channels will be used to probe various processes that occurs at the interface, from the hydrodynamic deformation of a pure interface to the monitoring of chemical reaction. Along this work, we will stress the importance of the interface characterization to better understand the mechanisms of stabilization of the interfaces in confined environment. 


\subsection{Thesis outline}

This thesis will is divided in two parts. In a first part we will review the general physical concepts which are required for the full understanding of the interface behavior in flow, such as the capillary forces and the kinetics properties of transport of the surfactant molecules to the interface. We will also detail the on-chip emulsification techniques used in our work, and the general background used for the characterization of hydrodynamics deformation in confined environments.

The second part will present the results obtained during this Ph.D. thesis. We will start with a chapter that reviews the experimental methods implemented in this work. A second chapter will be dedicated to the determination of the emulsification system, through the complete characterization of three surfactants in pendant drop method. The emulsification systems studied are perfluorosurfactants of practical interests for industrial and research applications. The third chapter describes the implementation of a microfluidics method used to measure the dynamic surface tension through the controlled deformation of the interface in a flow profile. In a fourth chapter, the deformation of the droplet in microfluidics will be detailed in order to establish a model for the droplet deformation in microfluidics. Here we will demonstrate that unlike in the unbounded flow, the droplet deformation in microchannels must take into account the confinement of the droplet, revealed by different scaling laws. We will also present in this chapter the deformation of the droplet in an inertial that is featured by interface oscillations. This regime provides additional insights into the surface tension measurement. The last chapter of this part will present an attempt to use the microfluidic technique to follow the dynamics of a polymerization reaction occurring at the interface. We will see that the treatment of the resulting viscoelastic interfaces requires the implementation of new tools and experimental methods. This work will be concluded by the possibilities that microfluidics can provide for such studies. 



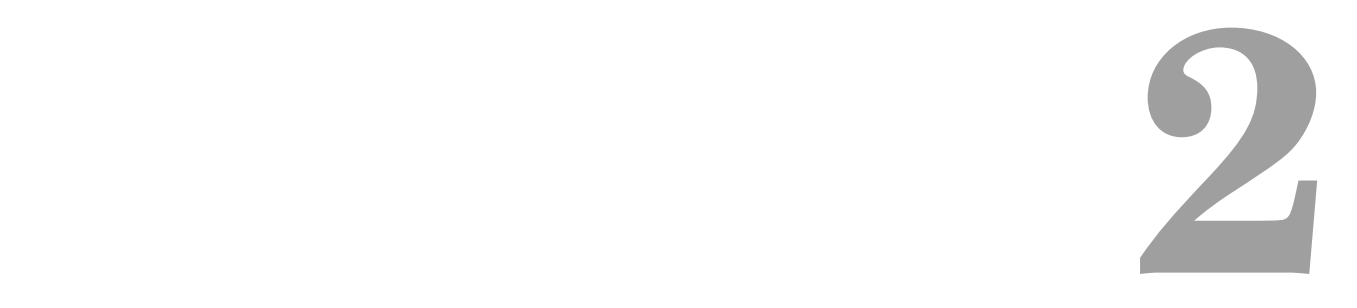

\section{Surface tension and capillary forces}

\subsection{Origins of the surface tension}

The mechanical properties of simple interfaces are mainly characterized by one quantity called the surface tension. In this section we present the origins of this quantity on the molecular scale, and the resulting forces that arise at macroscopic scale.

One can everyday make the observation that a volume of liquid or a solid, without additional energy will remain as cohesive ensemble. Therefore the molecules composing the material are subject to interactions that are sufficient to maintain a homogeneous compact structure. However molecular layers near the interface are a specific case and the presence of an other phase modifies the possibilities of interactions of the molecules.

Let us take the precise example of a water drop. In the bulk, the molecular interactions are dominated energetically by the hydrogen bonds [5], and one water molecules interacts, according to standard models, with four of its nearest neighbors. Close to the interface, the number of neighbor molecules is reduced and not all the possible bonds can be formed. As a result, the surface region is characterized by an excess of energy. This energy is represented in the expression of the internal energy of a system $U$ by the terms $\gamma A$ as following:

$$
U^{\mathrm{s}}=T S^{\mathrm{s}}+\gamma A^{\mathrm{s}}+\sum \mu_{\mathrm{i}} N_{\mathrm{i}}^{\mathrm{s}}-P V^{\mathrm{s}}
$$




\begin{tabular}{cc}
\hline Fluid & air/fluid surface tension $\gamma\left[\mathrm{mN} \cdot \mathrm{m}^{-1}\right]$ \\
\hline \hline Silicon oils & 20 \\
\hline Organic liquids & 50 \\
\hline Water & 72 \\
\hline Liquid metals & 700 \\
\hline Molten glass & 1000
\end{tabular}

Table 2.1 Surface tensions for the interface air / fluid for a selection of fluids. [8].

with, $P, V, T, S, A, \mu_{\mathrm{i}}, N_{\mathrm{i}}$ are respectively: the pressure, the volume, the temperature, the entropy, the surface area, the chemical potential and the number of particles of type $i$ of the system. The superscript $s$ indicates that the quantities are taken at the interface. In this representation the interface is the transition region between two phases and is considered to have a certain thickness, which explain the volume energy term $P V[6,7]$. The term $\gamma$ refers to the surface tension expressed in units of a force per unit of length $\left[\mathrm{N} \cdot \mathrm{m}^{-1}\right]$. This description applies to solid surfaces in contact with a gas or a liquid and also for liquid / liquid or liquid / gas interfaces.

The energies of the chemical bonds that compose the bulk phases are then linked to the values of the surface tension. For example, the hydrogen bonds that are present in water are ten times stronger than regular Van der Waals forces occurring in other common solvents[5]. They provide a larger surface energy for an equal area $A$. As a consequence the surface tension of an air / water interface is roughly 50\% higher than for other solvents. See table 2.1. For liquid metals like mercury, the covalent bonds ensure even higher surface tension. The direct consequence of the surface energy term in the equation 2.1 at macroscopic scales, can be shown by expressing the work $d W=d U+d Q$ needed to extend a surface $A$ by a quantity $d A$, where $Q$ is represents the heat exchanges. For conditions of constant temperature, pressure and amount of molecules at the interface we have:

$$
\left.d W\right|_{\mathrm{T}, \mathrm{P}, \mathrm{N}}=\gamma d A
$$

We see from equation 2.2 that the formation of an interface "costs" energy. A good way to exemplify this is to look at the shape of a drop at rest on a surface. In this case, the droplet takes the shape of a portion of a sphere[9]. This is true only for drops under a size defined as the capillary length, where the surface tension forces are not dominated by the gravitational 
forces that act on the volume. Here the principle of minimization of the interfacial energy, imposes that the amount of interface $A$ is minimized. In three dimensions, without boundaries and for a constant volume this surface is a sphere. It is possible to obtain spherical drops with large dimensions in state of weightlessness only, for example in the ISS where the gravity is canceled by the inertial momentum of the object. See figure 2.1

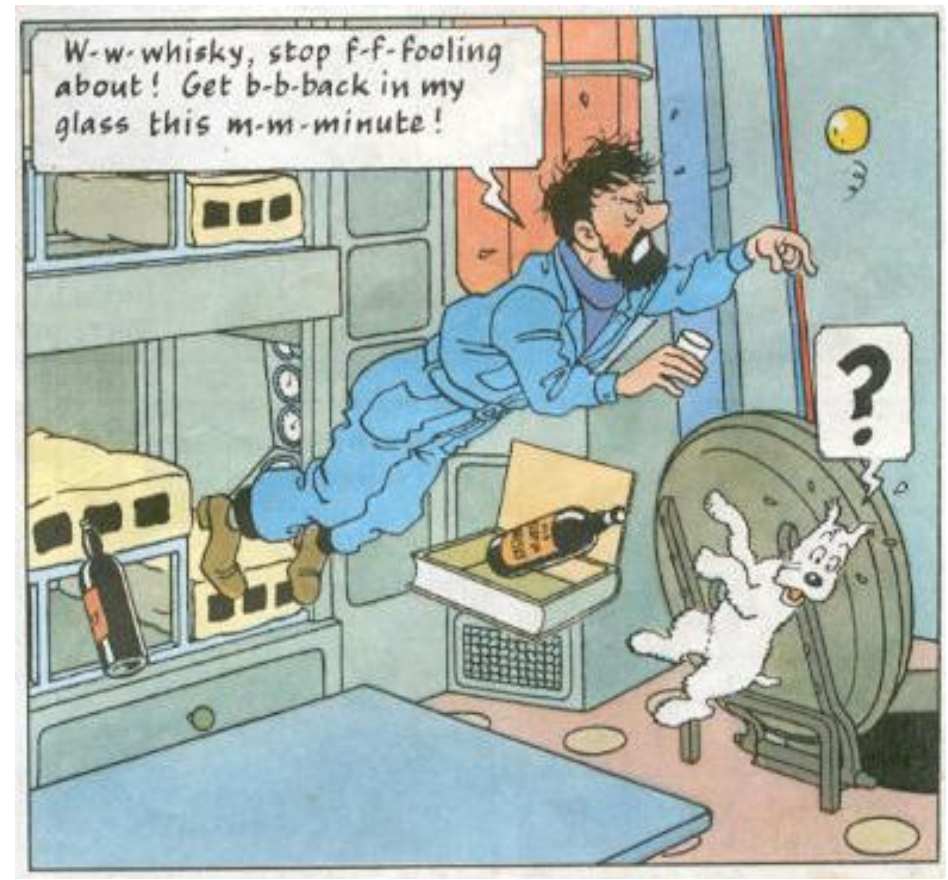

Figure 2.1 Shall Captain Haddock really be surprised by the shape that takes his Whisky drop in state of weightlessness ? Reprint from Tintin album "On a marché sur la Lune", Hergé, C)Moulinsart/Casterman, 1954

At macroscopic scale capillary forces that arise from surface energy and are presented in the following section.

\subsection{Capillary forces}

\subsubsection{Laplace pressure}

We have seen that the interfacial tension act on the interface to reduce the size of the interface. When integrated to the whole surface of an object these forces create an overall pressure that is equilibrated by the volume forces. As a consequence a pressure jump occurs through the 
interface. This pressure difference $\Delta P$ is proportional to the surface tension, and locally to the curvature of the interface. It is described described by the Gauss-Laplace equation:

$$
\Delta P=\gamma\left(\frac{1}{R_{1}}+\frac{1}{R_{2}}\right)
$$

Where $\Delta P=P_{1}-P_{2}$ is the difference between the external and the internal pressure. $\mathbf{n}$ is

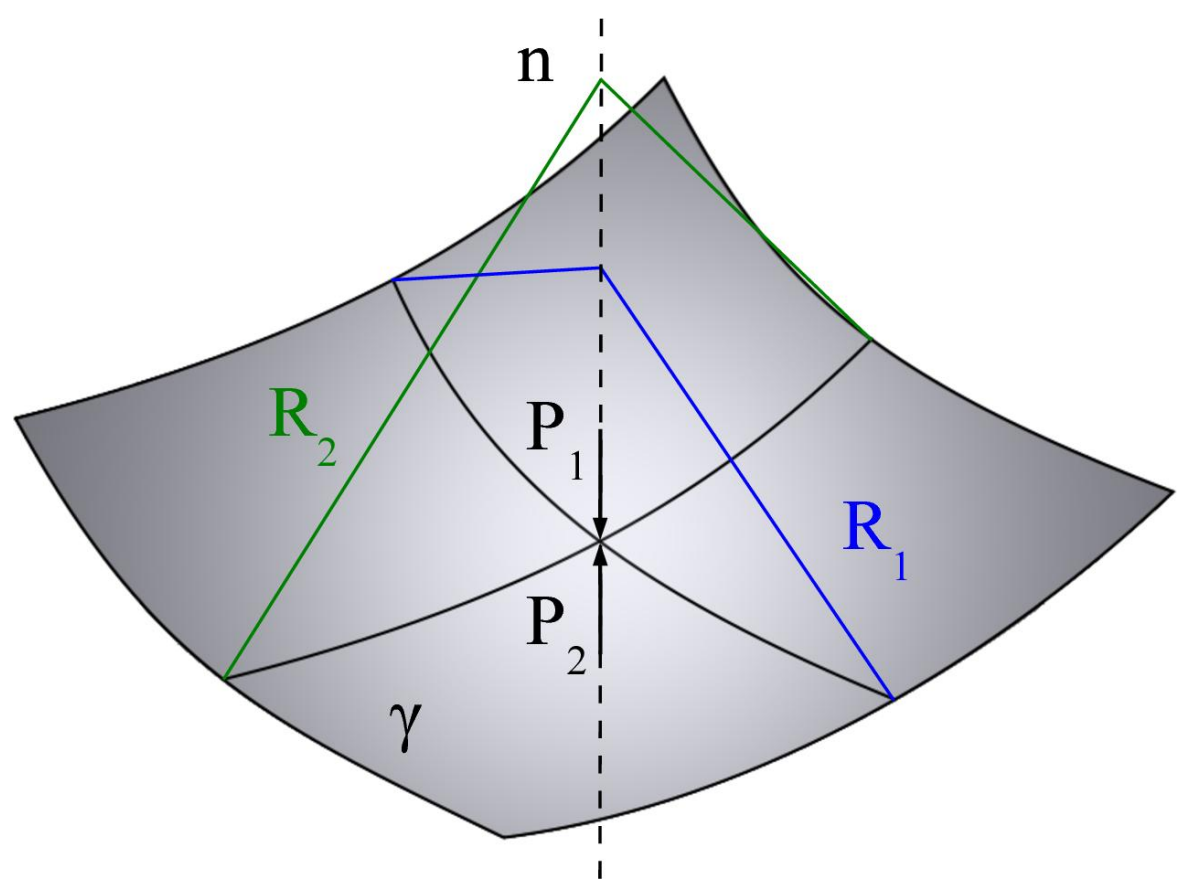

Figure 2.2 Illustration of the Laplace pressure on an element of surface defined by a surface tension $\gamma$. The two principal radii of curvature $R_{1}$ and $R_{2}$ determines the pressure jump $\Delta P=P_{1}-P_{2}$ through the interface.

the normal vector of the interface. $R_{1}$ and $R_{2}$ are the main radii of curvature at the point of the interface considered. See figure 2.2 .

For a free interface a homogeneous curvatures will ensure a homogeneous Laplace pressure. In presence of additional local forces on the interface, the curvature will be changed. If the magnitude of the force is know, the surface tension can be retrieved from the equation 2.3 . In the other way round if the surface tension is known, the measure of the deformation can be used to determine the force acting on the interface. 


\subsubsection{Contact line forces}

We consider now the case of a drop on a solid substrate which is immersed in a third fluid (gas or liquid). In this case a contact line appears at the point where the three phases meet. See figure 2.3 (a). At equilibrium the surface tension between the solid and the third fluid $\gamma_{\mathrm{s}}$, the surface tension between the liquid and the third face $\gamma_{1}$ and the surface tension between the liquid and the solid $\gamma_{1 / \mathrm{s}}$ are at equilibrium. They define the contact angle $\theta$ that the liquid will form with the substrate through the Young equation[10]:

$$
\gamma_{\mathrm{s}}-\gamma_{\mathrm{l} / \mathrm{s}}=\gamma_{\mathrm{l}} \cos \theta
$$
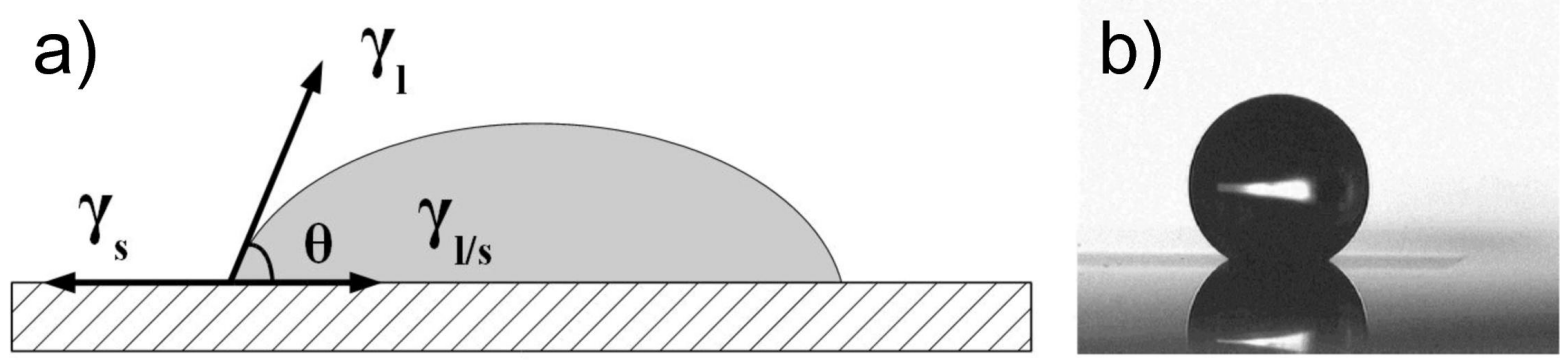

Figure 2.3 (a) Sessile drop on a plane surface. According to the Young equation (equation [10]) the equilibration of the three surface tensions $\gamma_{1 / s}, \gamma_{\mathrm{s}}$ and $\gamma_{1}$ at the contact line determines the contact angle $\theta$. (b) Water droplet deposited on a microstructured surface (visible under the droplet). The contact angle in that case approaches $\theta=180^{\circ}$, reprint from [11].

The values of $\theta$, is strongly linked to the balance between the surface energies of the solid and the two other phase. When the solid has more affinities for the liquid, the surface tension of the liquid is small in comparison to the surface tension of the third phase $\left(\gamma_{1 / \mathrm{s}}<<\gamma_{\mathrm{s}}\right)$ the droplet will spread on the surface an the contact angle will tend to $\theta \rightarrow 0^{\circ}$. It is usually the case for water droplets on glass surfaces. On the contrary if the surface has more affinity with the third phase $\left(\gamma_{1 / \mathrm{s}}>>\gamma_{\mathrm{s}}\right)$, the droplet will remain tangential to the interface and the contact angle will approach $\theta \rightarrow 180^{\circ}$. This is visible for water deposited on surfaces coated with a organic film, or for liquid deposited on microstructured interfaces [11]. See figure 2.3 (b). This property is also referred to wetting.

The force that originate from the formation of this contact line is proportional to the surface tension and can be described locally:

$$
d F=\gamma_{1 / \mathrm{s}} \cos \theta d l
$$


As an example the climbing of a meniscus on a vertical plane, will be driven by this force, the equilibrium being reached when the gravitational forces equilibrate the contact line force. The Jurin law solves this in the case of a capillary rise inside a tube[9].

\subsection{Methods of measurement}

The measurement of the surface tension of one interface is necessary for the characterization of the mechanical properties of the interface, and predicts its dynamic behavior. First, the value of the surface tension is a relevant quantity to predict the foaming / emulsification properties of a system of two non-miscible phases. Second, in presence of surfactant molecules at the interface, that modidy the surface tension, this measurement can be use as an estimation of the purity of a sample, as the presence of surface active impurities or dissolved electrolytes, in one of the two phases will modify the surface tension compared to the pure phases[12]. In addition, the dynamic measurement of adsorption itself provides information on kinetics properties of surface active molecules, such as the diffusion coefficient or the thermodynamic equilibrium constant. This point will be developed in the next chapter together with the description of the surfactant diffusion mechanisms to the interface.

A large panel of techniques have been developed to measure the surface tension. Most of them are implemented on millimetric or centimetric scale, and have been reviewed in[13], and are presented in figure 2.4(a) to (h).

Two essential physical principles are used for all these measurements techniques. First the shape techniques (or Laplace pressure techniques) which relies on the interface modification by an external force, and the measurement of the pressure jump across the interface. These methods are based on the Gauss-Laplace equation 2.3. where supplementary terms can be added to take into account the influence of external forces on the interface. This concept is used, for example, in the pendant drop technique described below. Second, comes the forces methods, where the measurement is based on the contact line forces described by the YoungLaplace equation (equation 2.4).

If correctly implemented all methods provide access to the dynamic surface tension. However the latency time between the time of the interface creation, and the beginning of the measurement varies. The surface tension measurements are extremely sensitive, therefore great care should brought to the preparation of the interface. The shape detection methods, can be implemented together with the synchronization between the droplet production and 
the measurement start, therefore the latency time of $\sim 10^{-2} \mathrm{~s}$ can easily be achieved and fast dynamics $\sim 1 \mathrm{~s}$ can be resolved. The figure 2.4 give an overview of the range of time scale covered by these methods.
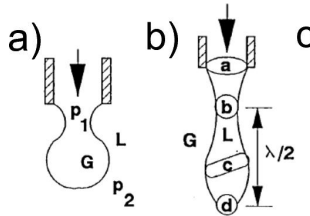

c)

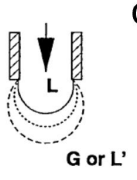

e)

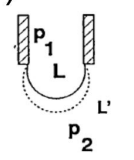

f)

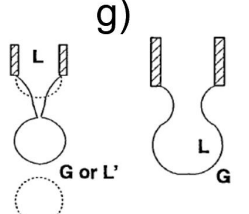

d)

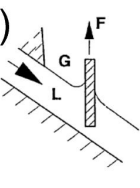

h)

G 原 $\boldsymbol{H}^{\mathrm{h}}$

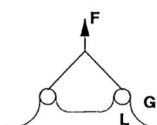

a) $\perp \quad$ maximum bubble pressure

b) Losc. jet

c) $\quad$ growing drop

d) Lincl. plate!

e) drop pressure g)

h)

drop volume

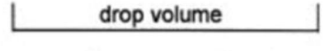

pendant drop

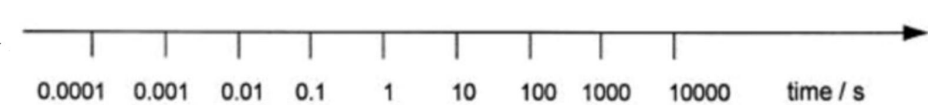

Figure 2.4 Schematic representation of various surface tension methods, and the corresponding time scales of application. Here the gas phases and liquid phase are represented by letters $\mathbf{G}$ and $\mathbf{L}, \mathbf{L}$ ' is used for an additional liquid phase. The methods depicted are: (a) maximum bubble pressure, (b) oscillating jet, (c) growing drop, (d) inclined plate, (e) drop pressure, (f) drop volume, (g) pendant drop method and (h) Whilhelmy plate together with Du Nouÿ ring. The two last techniques will be detailed at the end of this chapter. Reprints from[13, 14]

In the following part we will focus on two techniques that were used in this work for the measurement of the surface tension.

\subsubsection{Pendant drop tensiometry}

The pendant drop method relies on the measurement of the shape of a droplet determined by the gravity forces and the surface tension forces. Here the two forces are competing against each other. The surface tension will tend to preserve the spherical shape of the drop while gravity will induce a directional deformation of the interface. In practice a droplet is produced at the tip of a needle and the contour of the interface is extracted with image treatment methods [15]. The geometry of the problem is axisymetric. The contour shape of curve interface obeys the Gauss-Laplace equation 2.3. Therefore the extraction of the contour of the drop, in a plane cutting the drop through its axis of symmetry is enough to measure the surface tension. See figure 2.5. In the basic method the contour is parametrized as a function of the horizontal radius of curvature $R_{1}$.

$$
\frac{1}{R_{1}}=\frac{d \phi}{d S}
$$


and the azimuthal radius $R_{2}$

$$
\frac{1}{R_{2}}=\frac{\sin \phi}{x}
$$

where $S$ is the surface arc length, and $\phi$ the angle of the tangent to the interface. $x$ is determined by a referential centered on the droplet apex. See figure 2.5 .

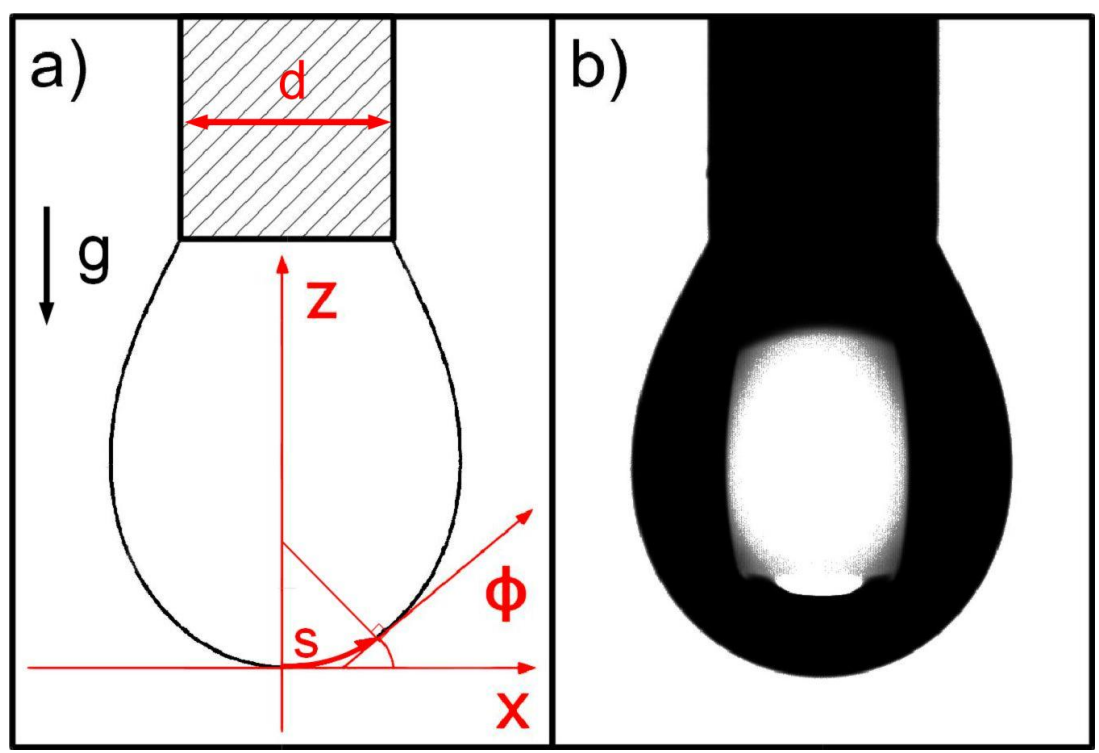

Figure 2.5 (a) Pendant drop profile with the parameters of interest for the contour extraction. (b) Corresponding experimental profile (drop of water in air). In both cases the scale of the picture is given by the needle outer diameter: here $d=1 \mathrm{~mm}$.

The pressure jump in a gravity field is given by:

$$
\Delta P=\frac{2 \gamma}{b}+\Delta \rho g z
$$

where $b$ is the radius of curvature at the tip of the droplet, $\Delta P$ is the pressure of the inner phase column on the point $X=Z=0$ of our figure. Bringing together the equations 2.6, 2.7, 2.8, and dividing the resulting equation by $\gamma / b$ one obtains:

$$
\frac{d \phi}{d S^{\prime}}=2+z^{\prime} B o-\frac{\sin \phi}{x^{\prime}}
$$

with the non-dimensional parameters

$$
S^{\prime}=\frac{S}{b}, \quad z^{\prime}=\frac{z}{b}, \quad x^{\prime}=\frac{x}{b}
$$


and the shape factor of the droplet that correspond to the Bond number $B o$ :

$$
B o=\frac{\Delta \rho g b^{2}}{\gamma}
$$

where $\Delta \rho$ is the difference in density between the two fluids that account for the buoyancy of he drop. $L$ is a characteristic length, in general the droplet radius and $\gamma$ is the surface tension.

The solution of 2.9 allow then the measurement of the surface tension $\gamma$ that is contained in the term $B o$. The solution is not analytical and the determination of $\gamma$ is done via the fitting of the drop contour and using the boundary condition $x^{\prime}(0)=0, z^{\prime}(0)=0$, and $\phi(0)=0$, for $S^{\prime}=0[15]$.

Usually the optical contour of the droplet can be extracted fairly easily with an image treatment method. However limitations for this method arise in the case of optical index matching fluids, where the interface can not be detected optically. The second limitation is the density matching phases, where the interface will not be deformed due to neutral buoyancy (the term $B o$ tends to 0 in the equation 2.9). In that case is it recommended to use force based measurements such as the Wilhelmy plate method.

\subsubsection{Whilhelmy plate tensiometry}

The Wilhelmy plate tensiometry is a force measurement method and requires the formation of a meniscus. The system is composed of a solid plate linked by a thread to a module measuring vertically applied forces. See figure2.6. The plate is most of the time made of roughened platinum or an absorbing material (paper) in order to ensure its perfect wetting. When the plate is brought in contact to the interface of interest, one phase will completely wet the plate surface and form a meniscus with an angle close to $\theta=0^{\circ}$. A contact line is formed with a length is given by the perimeter of the plate. The resulting force of the liquid on the plate is then derived from the equation 2.5 .

$$
F=2 \gamma(L+l) \cos \theta
$$

where $L$ is the length of the plate, and $l$ the width of the plate. In the case of perfect wetting, $\theta=0, \gamma$ is directly proportional to the measured force. If the wetting is incomplete, the value of $\theta$ should be measured to quantitatively link the force measured and the surface tension[16]. From the implementations times given in figure 2.4, we see that this technique is not able 

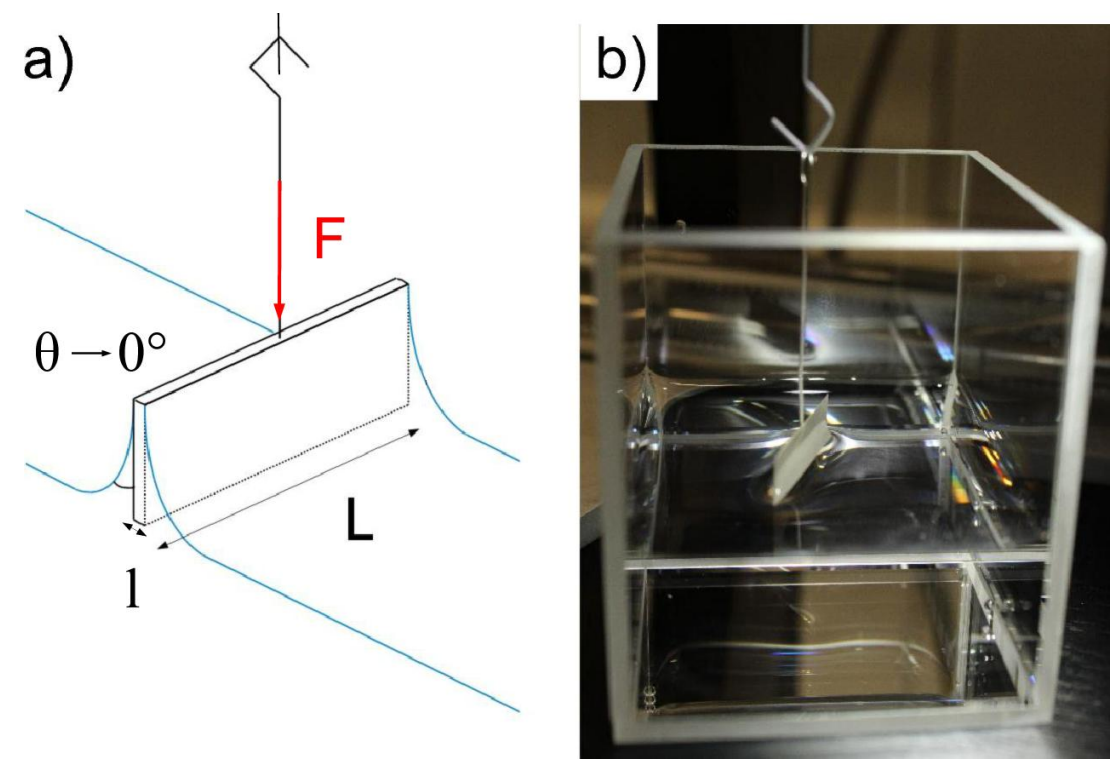

Figure 2.6 (a) Schematic display of the Wilhelmy plate experiment. The respective plate width and length are represented by $l$ and $L$. The contact angle of the water is given by $\theta$ and approaches $0^{\circ}$ ). (b) Experimental setup for an air / water interface. The platinum plate used for the measurement is visible with the meniscus.

to give efficient measurements of the dynamic surface tension below $\sim 100 \mathrm{~s}$. This part of the dynamics can be useful for the determination of the diffusion constant of a surfactant molecule in solution. The arguments will be presented in the following chapter dedicated to the description of tensioactive molecules and their mechanisms of adsorption to the interface. 


\section{Adsorption of surfactant to the interface}

\subsection{Surfactant molecules}

The capillary effects at an interfaces have a typical energy close to $\sim k T$, and are consequently extremely sensitive to small variations of temperature or composition of the interface. This is shown by the expression of the internal energy of the interface $U^{\mathrm{s}}$ defined in the previous chapter (equation 2.1). I this chapter we introduce the concept of surfactant molecules, that can adsorb spontaneously to an interface and consequently change its surface tension. In this chapter we also provide the background to understand the mechanisms of adsorption of these molecules to the interface. The models established in this chapter will be later used to characterize the kinetic properties of surfactant molecules.

In the nature a class of molecule possesses surface active properties, that is to say that the spontaneously adsorb and self-organize at an interface. These molecules also called amphiphile, are usually composed of two chemical parts with a strong affinity for two nonmiscible solvents. Most of the time the surfactant molecules are composed of a polar "head", which has a strong affinity for polar solvents like water and a "tail" consisting of a carbon backbone, that is mostly soluble into alcanes, figure 3.1 (a). Due to their chemical compositions the surfactant molecules will set preferentially at the interface. Some molecules are by nature excellent surfactants, this is namely the case for fatty acids or phospholipids. The 

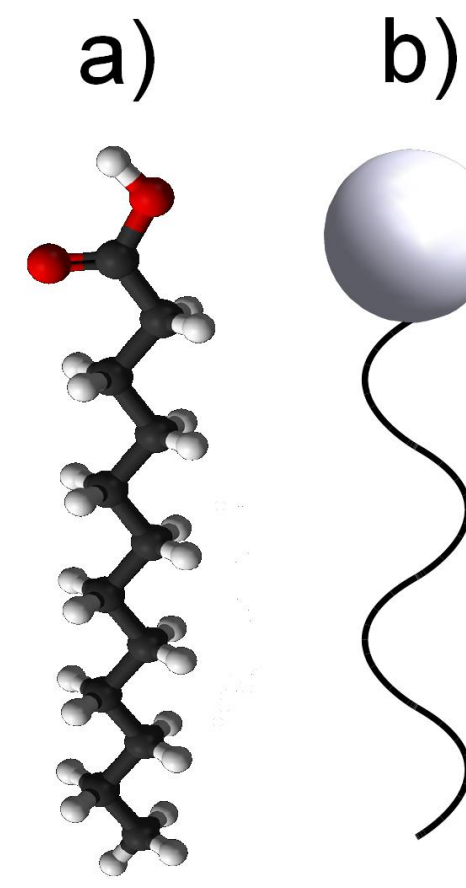

c)
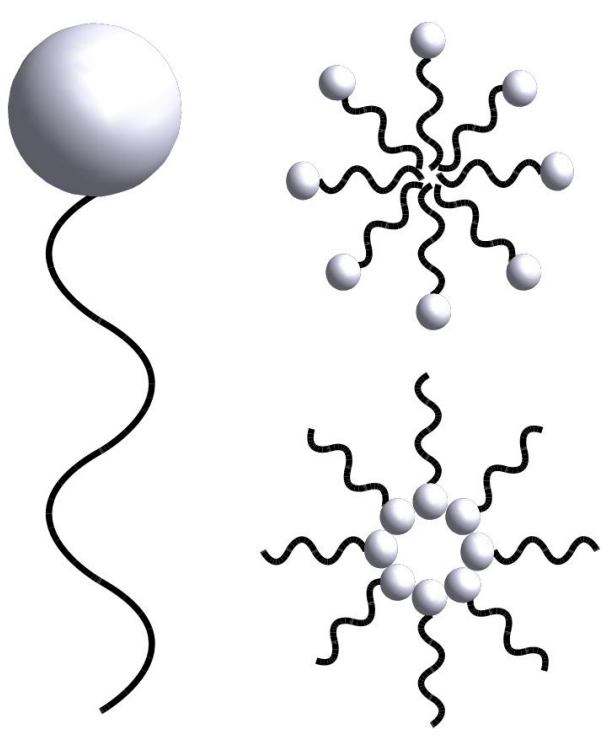

Figure 3.1 (a) Molecule of Lauryl acid, used as a base for the synthesis of many surfactant molecules of daily use Courtesy Wikimedia commons. (b) Schematic representation of a surfactant, with the polar head as a sphere and its alkyl chain. (c) Schematic representation of a micelle in the case the molecule is solved into water (top), and an inverted micelle, when the surfactant in solved in oil.

amphiphilic molecules are ubiquitous, for the large panel of applications that they provide. The surfactant molecules are used for foaming and emulsification, in cosmetic or food industry, and as detergents. A representative surfactant molecule is the sodium Laureth sulfate molecule that is, given its inexpensive way of production, common in most of the daily life products. Here the polar head is a charged sulfate group neutralized by a sodium cation. The lypophilic tail consists in a number of ethoxyl groups attached to a dodecyl chain. See figure 3.1 17, 18]. Furthermore the phospholipid molecules are essential to life, given the properties of self assembly in lipid-double layer that form basic living unit such as cells or vesicles.

\subsubsection{Amphiphiles classification: Charges \& HLB}

Depending on their physico-chemical properties, surface active agents can be easily classified. An obvious way is the distinction made by the charge they carry [18]. In addition to the steric repulsion, the additional electrostatic forces change dramatically the behavior of the surfactant 
molecules at the interface due to interaction between neighboring molecules. The surfactant molecule is cationic, when bearing one or several positive charges, anionic in the presence of negative charges and neutral when no charges are present. One can cite the category of the Zwitterionic surfactant that possesses two acid-basic group that change the overall molecular charge as a function of the $\mathrm{pH}$ of the solvent.

The concept of Hydrophilic-Lipophilic Balance (HLB) is introduced on the base of the partitioning of the surfactant molecule in aqueous or organic solvent. It is calculated via the following equation:

$$
H L B=7+\alpha \log (k)
$$

Where $k=C_{\mathrm{o}} / C_{\mathrm{w}}$ is the ratio between the concentration at equilibrium of the surfactant in the oil phase relative to the water phase, $\alpha$ is a scaling constant. Highly lipophilic molecules will have large HLB and hydrophilic molecule will have a small one. Typically the HLB depends on the molecule itself, and increases with the length of the alkyl chain. Note that this concept is valid only for molecule being soluble in oil or water. For oil /oil surfactant one has to go back to the more general notion of partition coefficient.

\subsubsection{CMC \& Micelization processes}

In solution, the surfactant molecules are constantly dispersed by the thermal agitation. However above a certain concentration, called the critical micellar concentration (CMC), the formation of aggregates in thermodynamic equilibrium with the population of monomers becomes favorable. These aggregates are called micelles. By convention we call inverse micelle the aggregate where the hydrophobic tail is pointing outwards, see figure 3.1.(c).

The micelles can be described by their aggregation number $N_{\text {agg }}$ which refers to the number of surfactant molecules per micelles. One main assumption in the process of micellization is that above the CMC, the monomer concentration is a constant. Therefore each additional molecule added in the bulk takes part in the formation of further micelles. In a first place the number of micelles increases with the surfactant concentration. At higher concentration the structure of the micelle changes from spherical objects to, for example, worm-like micelles. These changes in the structure have an impact on the rheology of the phase in which they are solved[19]. Different structures where resolved, at various water / surfactant volume ratio, by electron microscopy by Vinson and et al.[20]. A phases diagram of this changes is presented in figure 3.2 


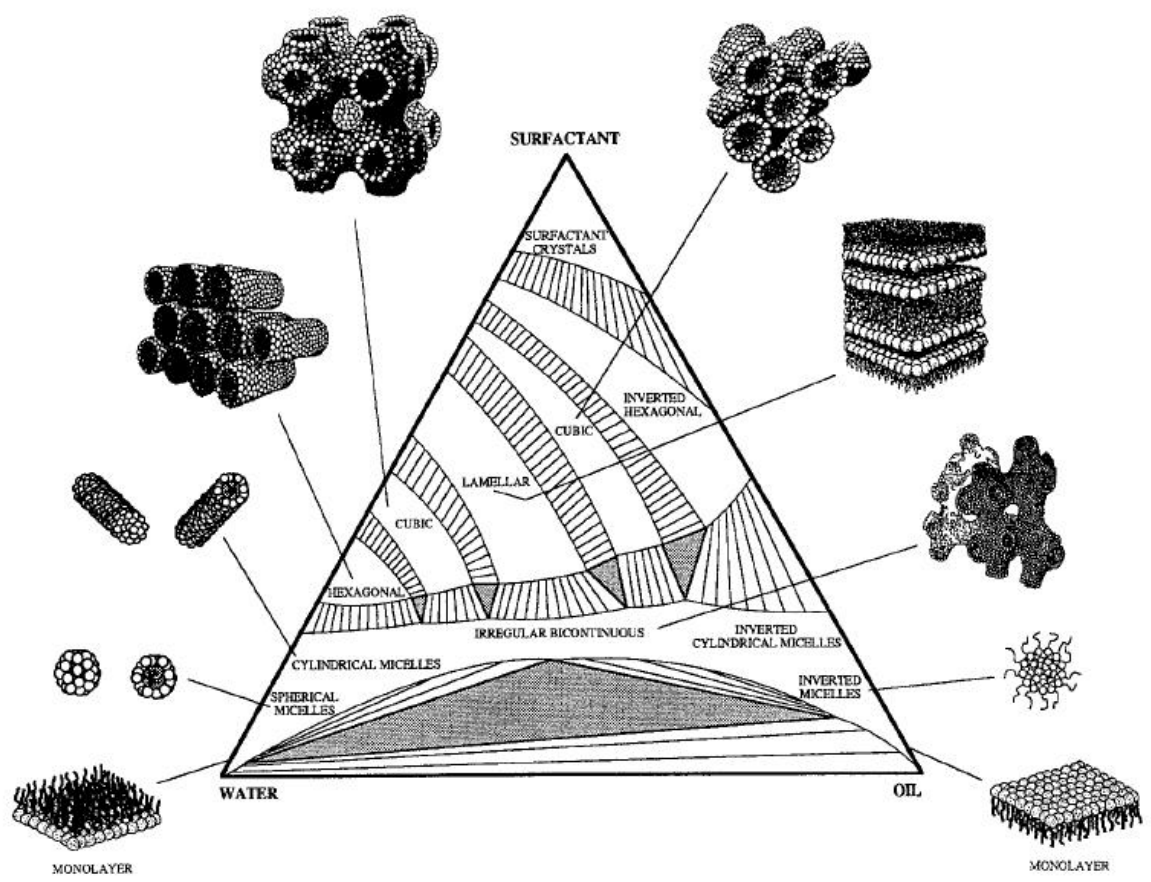

Figure 3.2 Phase diagram the micellar structure depending on the relative solvents-surfactant proportions. Reprinted from[20]

The equilibrium of a surfactant solution is determined by its chemical potential $\mu$ (itself linked to the chemical structure of the molecule), and by the external temperature $T$. The micelle of ionic molecules can exist above the Krafft temperature only, which is the temperature where the solubility of the molecule in a solvent becomes larger than the CMC.

The existence of the micelles depends on the chemical potential of the aggregates. In the Israelachvili description[21], the chemical potential of a surfactant molecule in solution is constant whenever existing as a monomer, or being part of a larger aggregate.

$$
\mu=\mu_{1}+k T \ln x_{1}=\ldots=\mu_{\mathrm{N}_{\mathrm{agg}}}+\frac{k T}{N_{\mathrm{agg}}} \ln \frac{x_{\mathrm{N}_{\mathrm{agg}}}}{N_{\mathrm{agg}}}
$$

which leads to:

$$
x_{\mathrm{N}_{\mathrm{agg}}}=N_{\mathrm{agg}}\left[x_{1} \exp \left(\frac{\mu_{1}-\mu_{\mathrm{N}_{\mathrm{agg}}}}{k T}\right)\right]^{\mathrm{N}_{\mathrm{agg}}}
$$

where $x_{\mathrm{i}}$ is the molar fraction of the solute in the aggregate, $k$ is the Boltzmann constant, and $\mu_{\mathrm{i}}$ the chemical potential. This equation gives a criterion for the micelle to monomer equilibrium, 
the stability of the aggregates being determined by $\mu_{1}>\mu_{N_{\text {agg }}}$. Ultimately the stability of the micelles depend on the way $\mu_{\mathrm{N}_{\mathrm{agg}}}$ evolves with $N_{\mathrm{agg}}$.

\subsection{Kinetics of surfactant adsorption}

In this section we review the principal models used in the description of surfactant adsorption at interfaces. We will first focus on the descriptions of an interface at equilibrium and introduce the concept of adsorption isotherms. Then we will present the theoretical description of the surfactant adsorption kinetics in the purely diffusional case, as a basic model. We will finish this review by presenting two further regimes beyond the diffusion model, also widely reported in the literature. Namely, a regime where convective transport is dominant, and the case where micellar aggregates are adsorbing at the interface.

We have seen that the adsorption of surface active molecules at the interface is decisive to ensure processes of foaming, emulsification, and detergency. Beyond the understanding of the physical mechanisms of the adsorption process, predicting the dynamics of adsorption of one surfactant, based on a panel of physical parameters intrinsic to the molecules, allows us to determine the efficiency of the aforementioned processes.

In the general case, the adsorption is seen as a dynamic exchange of surfactant molecules $A$ between the interface $I$ and the subsurface, where only adsorption is of importance for the early stage of the dynamics (few molecules at the interface), and where the desorption of surfactant gains in importance as the interface is covered. The dynamic equilibrium between the interface and the subsurface can be described by the equation:

$$
A+I \rightleftharpoons A I
$$

where $A$ represents a molecule in the bulk phase and $A I$ the molecule anchored at the interface. This model has been first introduced by Langmuir [22] and developed in a later paper on the adsorption of gases on planar solid surfaces [23]. The dynamics of adsorption depends on the concentration of surfactants in the bulk phase and in the subsurface. The mechanism of adsorption can be divided in two steps: (1) the transport of molecules (monomers or micelles) from the bulk to the subsurface, (2) the adsorption of the molecule from the subsurface to the interface [24]. Each step has an individual characteristic time $\left(\tau_{\text {transport }}\right.$ and $\left.\tau_{\text {adsorption }}\right)$. See figure 3.3 . The value of these time scales is affected by the local physical processes, for instance 


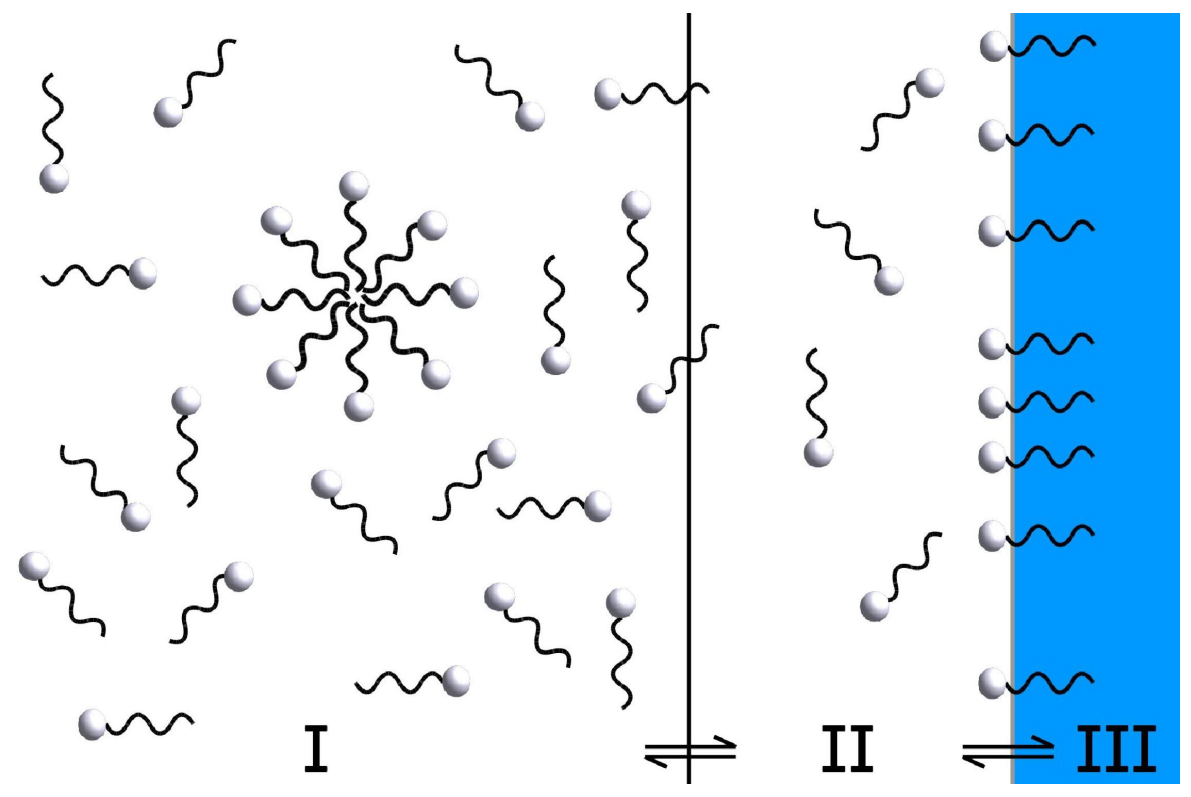

Figure 3.3 Schematic view of an interface with surfactant molecule adsorbed. The three region are separated. (I) Solvent bulk phase, (II) subsurface, (III) interface (magnified). The equilibration between the phases are represents by bi-directional arrows.

$\tau_{\text {transport }}$ depends on the diffusion coefficient of the surfactant molecule in the solvent, but will be strongly modified by the presence of convection in the continuous phase[25]. $\tau_{\text {adsortpion }}$ can be modified by the formation of an potential barrier at the interface in the case of adsorption of charged molecules[26]. Further refinements can be added, for example in the case of the dissociation of micelles into monomers [24, 27], or when the desorption of the surfactant from the interface is prohibited.

The comparison of these characteristic times defines two adsorption regimes, where the longer time is considered as being the limiting step. A first one is the diffusive-limited regime for $\tau_{\text {transport }}>\tau_{\text {adsorption }}$, and a second one is the transfer-limited regime for $\tau_{\text {transport }}<\tau_{\text {adsorption }}$.

\subsection{Equilibrium equation of state}

In order to characterize the thermodynamical properties of the reaction described by equation 3.4, it is convenient to focus on the equilibrium state of an interface, in presence of a concentration $C$ of surfactant in the bulk. It is then necessary to establish a quantitative link between the amount of surfactant present at the interface, and the surface tension.

One relation between the surface tension and the number of molecules present at the inter- 
face has been derived by Gibbs [14], and is based on the continuity of the chemical potential at the interface.

The starting point is the expression of the interface internal energy $U$ that has been defined previously (equation 2.1). The following derivation follows the one of Levin et al. [7].

$$
U^{\mathrm{s}}=T S^{\mathrm{s}}-P V^{\mathrm{s}}+\gamma A^{\mathrm{s}}+\sum \mu_{\mathrm{i}}^{\mathrm{s}} N_{\mathrm{i}}^{\mathrm{s}}
$$

The derivation of the Gibbs free energy $G^{\mathrm{s}}$ is defined through the Legendre transform

$$
G^{\mathrm{s}}=U^{\mathrm{s}}+P V^{\mathrm{s}}-\gamma A^{\mathrm{s}}-T S^{\mathrm{s}}
$$

which after substitution in equation 3.5 gives the Gibbs free energy $G^{\text {s: }}$

$$
G^{\mathrm{s}}=\sum \mu_{\mathrm{i}}^{\mathrm{s}} N_{\mathrm{i}}^{\mathrm{s}}
$$

The differentiation of equation 3.5 leads to the Gibbs-Duhem equation, that is written for $i$ species present at the interface. Here the surfactant and the solvent, where the volume term $V^{\mathrm{s}} d P$ is neglected[16].

$$
S^{\mathrm{s}} d T+A^{\mathrm{s}} d \gamma+\sum d \mu_{\mathrm{i}}^{\mathrm{s}} N_{\mathrm{i}}^{\mathrm{s}}=0
$$

leading to:

$$
d \gamma=-\left(S^{\mathrm{s}} / A\right) d T-\sum \Gamma_{\mathrm{i}} d \mu_{\mathrm{i}}
$$

with $\Gamma_{\mathrm{i}}=N_{\mathrm{i}}^{\mathrm{s}} / A$ is the surface excess of the component $i$. Now writing the variation of the chemical potential as $d \mu_{\mathrm{i}}=R T d \ln C_{\mathrm{i}}$ at constant temperature, where $C_{\mathrm{i}}$ is the concentration of the component $i$ and $R$ is the gas constant, we derive[16]:

$$
-\frac{d \gamma}{R T}=\sum\left(\Gamma_{\mathrm{i}} d \ln C_{\mathrm{i}}\right)
$$

The Gibbs convention imposes that $\Gamma_{\text {solvent }}=0$, so equation 3.10 leads to:

$$
\Gamma=-\frac{1}{n R T}\left(\frac{d \gamma}{d \ln C}\right)_{\mathrm{T}}
$$

where $C$ is the concentration of the surfactant. We introduce the constant $n$ linked to the dissociation of charged electrolytes at the interface, where $n$ is 1 for neutral molecules or ionic surfactants in the presence of excess electrolyte in the bulk phase, and $n$ is 2 for ionic 
surfactants in absence of electrolytes[14].

The Gibbs equation obtained gives then the basic relation between the surface mass excess of a molecule at the interface $\Gamma$ and the concentration of this surfactant in the bulk $C$. This equation is then used to describe the equilibrium adsorption isotherms.

\subsection{Adsorption isotherms}

We are interested now to express the surface tension of $\gamma_{\mathrm{eq}}$ as a function of the bulk surfactant concentration $C$. This equation of state, called adsorption isotherm, provides useful information on the molecules such as the CMC and the adsorption constant.

\subsubsection{Langmuir Isotherm}

In the Langmuir model we assumes an interface to be divided in a grid with a finite number $\Gamma_{\infty}$ of adsorption sites and allowing the adsorption of one molecule per site. The adsorption is equally probable and equivalent on all these sites, no matter of the state of occupancy of the neighboring sites. The equilibrium number of sites occupied $\Gamma$ can be calculated by the equation:

$$
\frac{\Gamma}{\Gamma_{\infty}}=\frac{\kappa C}{1+\kappa C}
$$

The parameter $\kappa$ is a reaction constant. The quantity can be expressed as the ratio between the molecules rate of adsorption $k_{\text {ads }}$, and rate of desorption $k_{\text {des }}$.

$$
\kappa=\frac{k_{\mathrm{ads}}}{k_{\mathrm{des}}}
$$

No interaction between neighboring molecules is explicitly mentioned but are de facto embedded in the constant $\kappa$ and $\Gamma_{\infty}$, that would change, for instance in the case of screening of neighboring charges of surfactant molecules. The surface tension is assumed to be proportional to the quantity of surfactant adsorbed at the interface. Therefore, the equilibrium expression of the interface given by equation 3.12 can be modified to recover the equilibrium surface tension $\gamma_{\mathrm{eq}}$. This is done with the help of the Gibbs equation 3.11, giving the relation between the surface coverage and the surface tension. The resulting equation is the von Szyszkowsky equation [28].

$$
\Pi=n R T \Gamma_{\infty} \ln (1+\kappa C)
$$


The quantity $\Pi=\gamma_{0}-\gamma_{\mathrm{eq}}$, is called the surface pressure, as the results of the change of Laplace pressure (equation 2.3 between the fresh, and the equilibrated interface.

\subsubsection{Henry Isotherm}

The Henry isotherm is one limiting case of the Langmuir model, that holds for the description of a dilute interface. Therefore this model does not describes the concentrations above the $\mathrm{CMC}$, where the interface is close to saturation. Here the quantity of surfactant at the interface is directly proportional to the concentration. It takes then the form:

$$
\Gamma=\kappa C
$$

The surface pressure can be recovered using equation 3.11 .

$$
\Pi=n R T \Gamma
$$

\subsection{Diffusive model for surfactant to the interface}

The dynamics of surfactant adsorption can be derived from the diffusion theory. The theory derived by Ward and Tordai in 1946 [29] has proven to be a robust model, for the of adsorption of neutral surfactants to the interface. The model is based on a Fickian diffusion expressed in its one-dimensional form by:

$$
\frac{\partial n}{\partial t}=D \frac{\partial^{2} n}{\partial x^{2}}
$$

Where $D$ is the diffusion coefficient of the molecule, and $x$ a length.

The model predicts the concentration of surfactant molecules at the interface. It takes into account the existence of a subsurface described above and the interactions between the interface and the subsurface, as well as the bulk and the subsurface. Figure 3.4 (a) shows successive steps during the process of adsorption. In this picture the dashed line draws the imaginary frontier between the bulk (left side), and the subsurface (right side). The hatched region symbolizes the interface. Each colored curve represents a significant step in the adsorption. They follow each other chronologically: (1) No adsorption has occurred, the bulk and subsurface are at equilibrium. (2): The early stage of the adsorption, the subsurface is depleted from its surfactant upon rapid adsorption to the interface. the steps (3) and (4) describe the intermediate times where the subsurface is being replenished by diffusion of the surfactant from 
the bulk phase. The adsorption to the interface is still occurring at this stage. (5) The final step where the dynamic equilibrium is established between the interface and the subsurface. In figure 3.4 (b) we represent an idealized dynamic surface tension as it can be measured in dynamics interfacial tensiometry. The described steps (1-5) are placed approximatively along this curve in order to make a connection with the surface coverage.

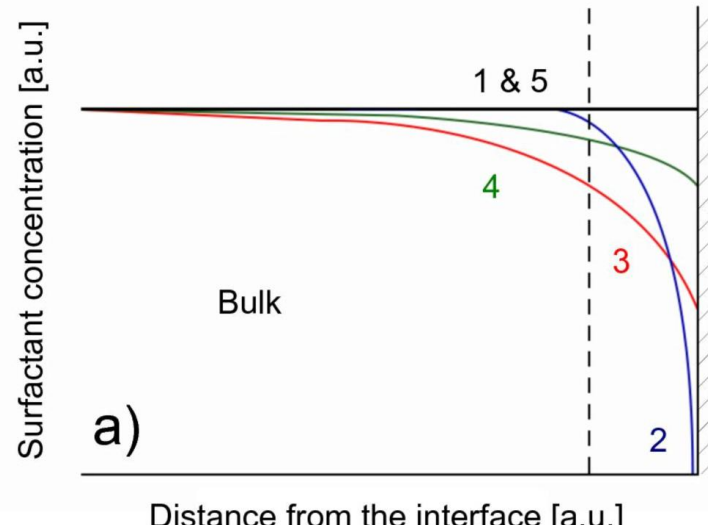

Distance from the interface [a.u.]

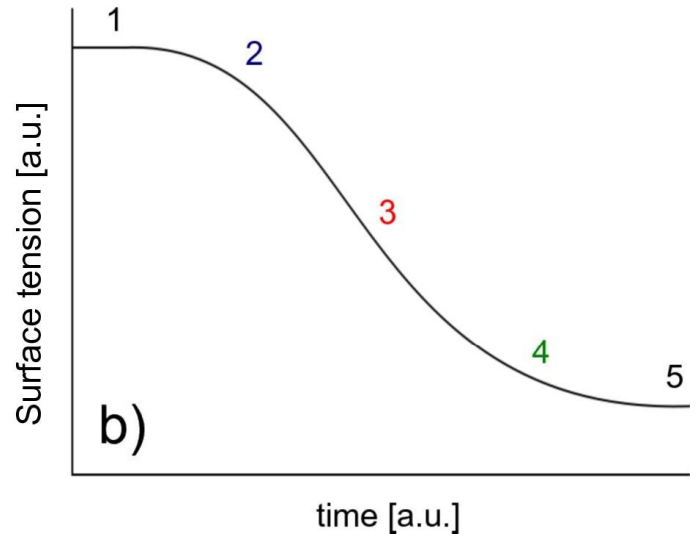

Figure 3.4 (a) Successive steps used to built the Ward and Tordai model. The interface is symbolized by the hatched region. The subsurface extends from the interface to the dashed vertical line. Beyond is the bulk region. Adapted from [29]. (b) Schematic of a dynamic surface tension curve as it can be measured by dynamic tensiometry. The numbers corresponds to the steps described in (a).

The process above is completely described by the following equation:

$$
\Gamma(t)=2 C \sqrt{\frac{D t}{\pi}}-2 \sqrt{\frac{D}{\pi}} \int_{0}^{\sqrt{t}} C_{\mathrm{s}} d(\sqrt{t-\tau})
$$

Where $C_{\mathrm{s}}$ is the concentration of surfactant in the subsurface region defined above, being a function of time, $\tau$ is a variable used in the convolution integration. The integration member on the right, accounts for the back diffusion of the molecules into the subsurface. This equation can not be solved analytically unless having a additional function that describes the concentration $C_{\mathrm{s}}$ with time. Unfortunately no general analytical description of $C_{\mathrm{s}}$ is available, which makes it difficult to solve the Ward and Tordai equation in the general case. 


\subsubsection{Solution of the diffusion equation in the asymptotic cases}

One strategy to solve the equation 3.18 is to consider the asymptotic cases $t \rightarrow 0$ and $t \rightarrow \infty$, respectively for a fresh interface, and an interface reaching its equilibrium, see figure 3.4 (b). The scaling law for such behavior has been derived by Fainerman et al.[30].

For the early kinetics, the integral over $C_{\mathrm{s}}$ in equation 3.18 disappears, as the back diffusion of the monomers from the interface can be neglected. We can then write:

$$
\Gamma(t)=2 C \sqrt{\frac{D t}{\pi}}
$$

At this stage of adsorption, one can assume the surface to satisfy the conditions of a diluted interface. It is possible to combine the equation 3.20 with the Henry isotherm. This leads to the expression of the dynamic surface tension at early times:

$$
\gamma_{0}-\gamma(t)=2 n R T C \sqrt{\frac{D t}{\pi}}
$$

The scalings of the late kinetics are derived as follow. Here the interface is considered to be at equilibrium with the subsurface. Additionally at infinite time the subsurface concentration tends to an equilibrium with the bulk $C \rightarrow C_{\mathrm{s}}$ for $t \rightarrow \infty$ and is a constant that can be taken out of the integral. The equation can be rewritten:

$$
\Delta C_{\mathrm{t} \rightarrow \infty}=C-C_{\mathrm{s}}=\Gamma \sqrt{\frac{\pi}{4 D t}}
$$

The interface at equilibrium can not be considered as diluted anymore, and the Gibbs equation is used to describe the variation of the surface tension with the coverage. The dynamic surface tension at infinity can then be described as:

$$
\Pi=\frac{n R T \Gamma_{\infty}^{2}}{C} \sqrt{\frac{\pi}{4 D t}}
$$

The two equations 3.20 and 3.22 , although being confined to specific time regions, can be easily compared to experimentally measured kinetics. The main limitation of these equations comes from the fact that they are applicable for diffusive cases of surfactant towards a planar interface. Thus refinements have to be added when considering for instance a curved interface[31], or the adsorption of charged molecules[26]. 


\subsection{Further transport models}

In this last part, we present further models which describe other modes of surfactant transport to the interface.

\subsubsection{Convective transport}

When the transport of the surfactant to the subsurface is dominated by convective effects, the time scale $\tau_{\text {transport }}$ becomes negligible in comparison to the adsorption time. Here, the subsurface is constantly replenished with surfactant and equilibrium with the bulk $\left(C_{\mathrm{s}}=C\right)[25]$. Furthermore, the boundary conditions of the continuity of the chemical potential expressed in the Gibbs equation is replaced by a kinetic equation describing the equilibrium interface[32].

$$
\frac{d \Gamma}{d t}=-k\left(\Gamma-\Gamma_{\mathrm{e}}\right)
$$

which after integration and with the assumption that the surface tension is proportional to the occupancy, yields.

$$
\gamma_{0}-\gamma(t)=e^{-\mathrm{t} / \tau}
$$

Here the equilibration depends on one parameter $\tau$ which is a characteristic time that takes stochastic processes at the molecular level into account for the adsorption. Therefore is embedded in this term the orientation of the molecules in the direction of the interface, the time it takes for the molecule of surfactant to find an empty site, as well as the desorption and adsorption constant. In the case of the adsorption of a charges surfactant the formation of the electrostatic barrier formed at the interface upon adsorption can lead to a transfer-limited regime [26].

\subsubsection{Micellar adsorption}

Above the CMC, the solute in the bulk is distributed into monomers and micelles. In diffusive processes, the size of the micelles render their diffusion coefficient much smaller than the monomer. Therefore, in presence of a small micelle population, the direct micelle adsorption at the interface is statistically unlikely, and practically not measurable. However, for concentrations far above $\mathrm{CMC}(\mathrm{C}>5 \mathrm{CMC})$ the large number of micelles present makes their influence no longer negligible for the adsorption processes. The adsorption has been 


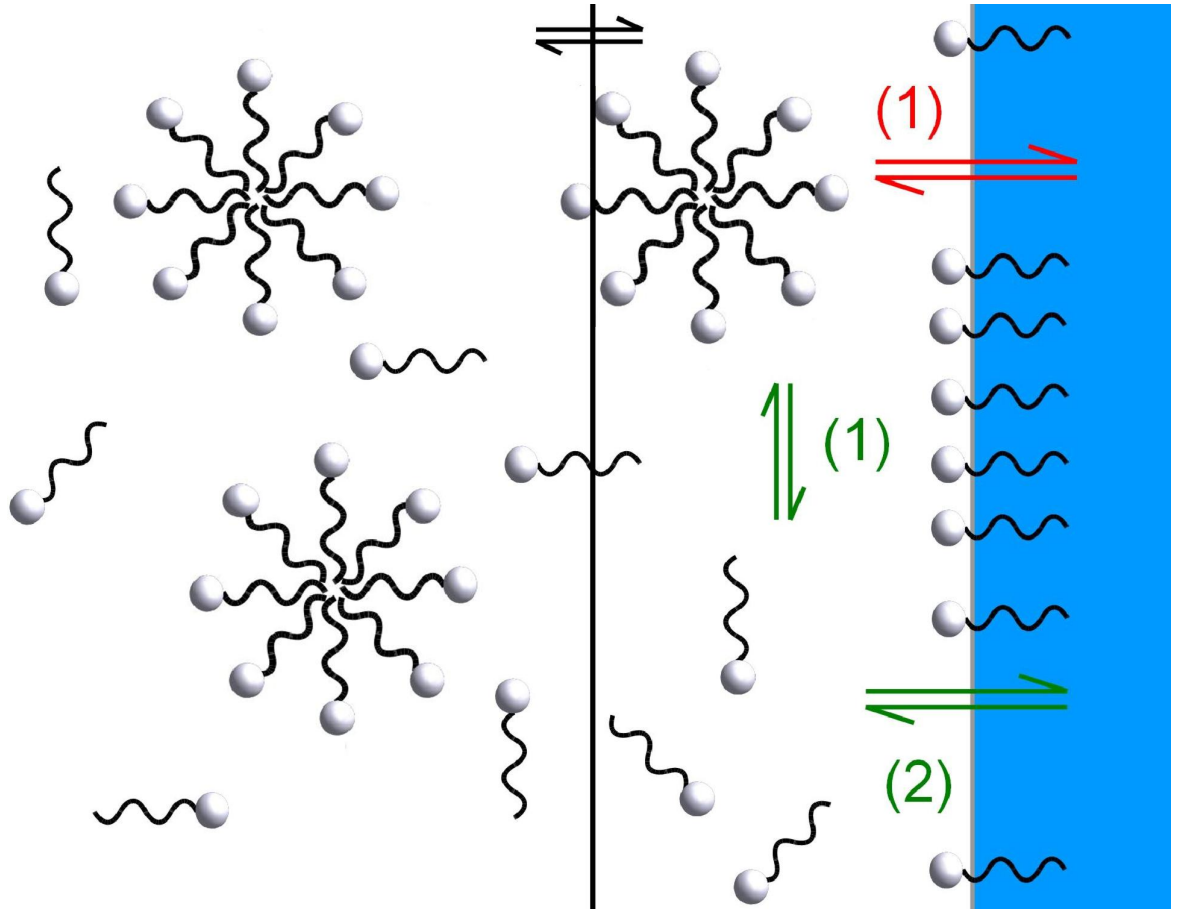

Figure 3.5 Schematic view detailing both processes for micelles adsorption. In green the two step process with the desegregation of the micelles in monomer before (2) their adsorption on the interface. The red arrows is the direct adsorption / desorption of the micelles to / from the interface. Note that the diffusion of the surfactant from the bulk to the subsurface is still present and has an importance in the process.

theorized [27] and two pathway for the adsorption considered.

First a two step process (green numbers in the figure 3.5). Here the breaking up of the micelles into monomers occurs in a surfactant-depleted subsurface (1). The monomers then adsorb to the interface (2). If the time of desegregation is long in comparison to the adsorption process, typically for the early kinetics of adsorption. Then the adsorption can be described by the asymptotic scaling that have been derived above but taking into account the concentration of the monomers in the bulk only. In the contrary, the kinetics of desegregation has to be taken into account in the diffusion equation, via the introduction of an effective diffusion coefficient, which takes into account the micelles as a source / sink of monomer.

The second path is a one-step process, (red numbers in the figure 3.5) where the direct adsorption of micelles at the interface is allowed. The possibility of this regime was predicted theoretically by Song et al.[24] in a model where the concentration of micelles is large enough so that the flux of aggregates can penetrate the subsurface without breaking up, and thus directly contribute to the adsorption. This regime was resolved optically using fluorescent track- 
ing of the micelles in the bulk. A micelles-free zone in the subsurface was resolved around the $\mathrm{CMC}$, that was filled with aggregates for concentration $\mathrm{C} \sim 5 \mathrm{CMC}[33]$. The significant role of the direct micelle adsorption to the interface was also demonstrated experimentally by Colegate et al. with a system of $\mathrm{C}_{14} \mathrm{E}_{8}$ at an air / water interface[34]. This work demonstrated in addition the impossibility of charged micelles to adsorb directly at the interface.

We have seen in this chapter how the surface active molecule adsorb at the interface and the scaling linked to diffusive models. These models will be used later in this work for the characterization of different surfactant molecules in pendant drop method as well as in microfluidics. In the following chapter we will describe the implementation of the surfactant molecules in microfluidics, for the stabilization of emulsions. 


\section{On-chip emulsification and hydrodynamics in microfluidic environment}

In our study we will use extensively the microfluidic environment and more particularly the techniques of emulsion production and interface stabilization, for the development of a new tool dedicated to interfaces characterization. We will also try to better understand the influence of the confinement on the overall properties of the droplet deformation. The objective of this chapter is then to give a background knowledge in the field of the on-chip emulsification, and in flows characterization in microfluidics for a better understanding of the following studies.

In the first part we will give a short general background on the development and the history of on-chip emulsification and their application in research today. Then we will present the techniques of on-chip emulsification and illustrate them with geometries that are commonly used in microfluidics. Then we will address the problem of the emulsion aging, and the techniques which are implemented to stabilize the interfaces and prevent the aging of the emulsion. The last part will be dedicated to the characterization of flows in microfluidics environment. We will derive from the Navier-Stokes equations the different scalings and the non-dimensional quantities that are relevant for the study of confined flow. 


\subsection{Introduction}

The use of microfluidics in soft matter studies is now ubiquitous. Since the beginning of the year 2000 the techniques of microfabrication of microfluidics chips derived from the solid state microfabriction techniques have been in constant improvement. First the development of the soft-lithography techniques, and the better resolutions grants a larger diversity in the geometry that can be produced. Second the development of the protocols for the Polydimethysiloxane (PDMS) chip production, provides an inexpensive, chemically inert, confined, environment of study.

Intrinsically the dimensions in microfluidics are pretty well suited for the study of objects like active or passive colloidal particles, with sizes ranging from nanometric beads to living cells. In addition the confinement in the microchannels provides a controlled environment for the study of the solution and the monitoring of the chemical reactions.

Microfluidic has proven to be a suitable tool in an extensive amount of domains. One can cite the following of chemical reaction with the use of coflow of two reagent phases and mixing [35, 36]. Microfluidics can also be applied in the study of particle auto-organization in microflow, as well as the particle sorting, with the use of external fields[37]. More recently one can note the emergence of topological microfluidic, dealing with the flow of liquid crystals and the organization of defect lines in confined environment[38].

The study of the two phase flow in microfluidics have lead to the production of the first droplet-based systems. From that point, the development of droplet-based applications have grown quickly taking the advantage of the droplet as microreactor. Here the droplet is used as a controlled environment enabling the implementation of quantitative analysis for chemistry and biology [36] like drug screening, or the monitoring of confined chemical reactions. The droplet environment is also used for microbiological application by encapsulating living organisms [39]. An important side effect of the compartmentalization is the large presence of the interface and the capillary effects which derive from it. The microfluidics environment is then suitable for the study of the mechanical properties of the interfaces in a two phases flow regime. These studies have required the development of reliable on-chip techniques of emulsification and foaming[4], and the control of the created interfaces. In the following section we will present some common on-chip emulsification techniques. 


\subsection{Principles of on-chip emulsification}

The general principle of on-chip emulsification lays in the dispersion of one fluid (dispersed phase) into a carrier fluid (continuous phase) to which it is non miscible, and in the stabilization of this dispersion by controlling the interfacial energy.

Specific geometries have been developed with that aim. In all the cases the flow of the continuous and dispersed fluids is modulated to provoke instabilities at the interface, and eventually the break up of the disperse phases in stable droplets. On-chip emulsification relies in addition on the control of the wetting properties of the channel. Chemical coatings are used to control the hydrophilicity / hydrophobicity of the walls.

The first presented geometry is the T-junction geometry. In this cases the channel that convey the dispersed phase meets a main channel conveying the continuous phase at a right angle. See figure 4.1 (a). The description of the mechanism as proposed by Garstecki et al. [40] is the following. As the dispersed phase obstructs the flow in the continuous phase in the main channel, the pressure rises upstream. The dispersed phases grows in the main channel and forms a neck. At a critical pressure the surface tension forces at the neck are overcomed, and interface breaks up. The droplet is released and flows downstream away from the junction. The cycle repeat as the dispersed phase starts to re-invade the main channel. This description allows the determination of the frequency of the droplet production and the volume of the droplet with the following scaling law.

$$
L / w=1+\alpha \frac{Q_{\mathrm{cont}}}{Q_{\mathrm{disp}}}
$$

where the $L$ is the length of the produced droplet, $w$ is the size of the channel. Respectively $Q_{\text {cont }}$ and $Q_{\text {disp }}$ are the flow rate of the continuous phase and the dispersed phase, and $\alpha$ is a constant of order one. The produced droplets are larger than the channel size. Such droplet are confined in the channel and are referred to as plug and exhibit specific flow properties[41].

The second method to produce droplets is the flow focusing junction, which has been for the first time described by Anna et al.[43]. In this geometry, two straight channels join at a right angle. See figure 4.1(b). The stream of dispersed phase emerge from one channel and the two perpendicular channels provide the continuous phase, both with the same flow rate to ensure symmetry of the droplet production. Both phases are then forced to flow into the remaining channel where an elongational velocity profile induces the focusing of the stream. 

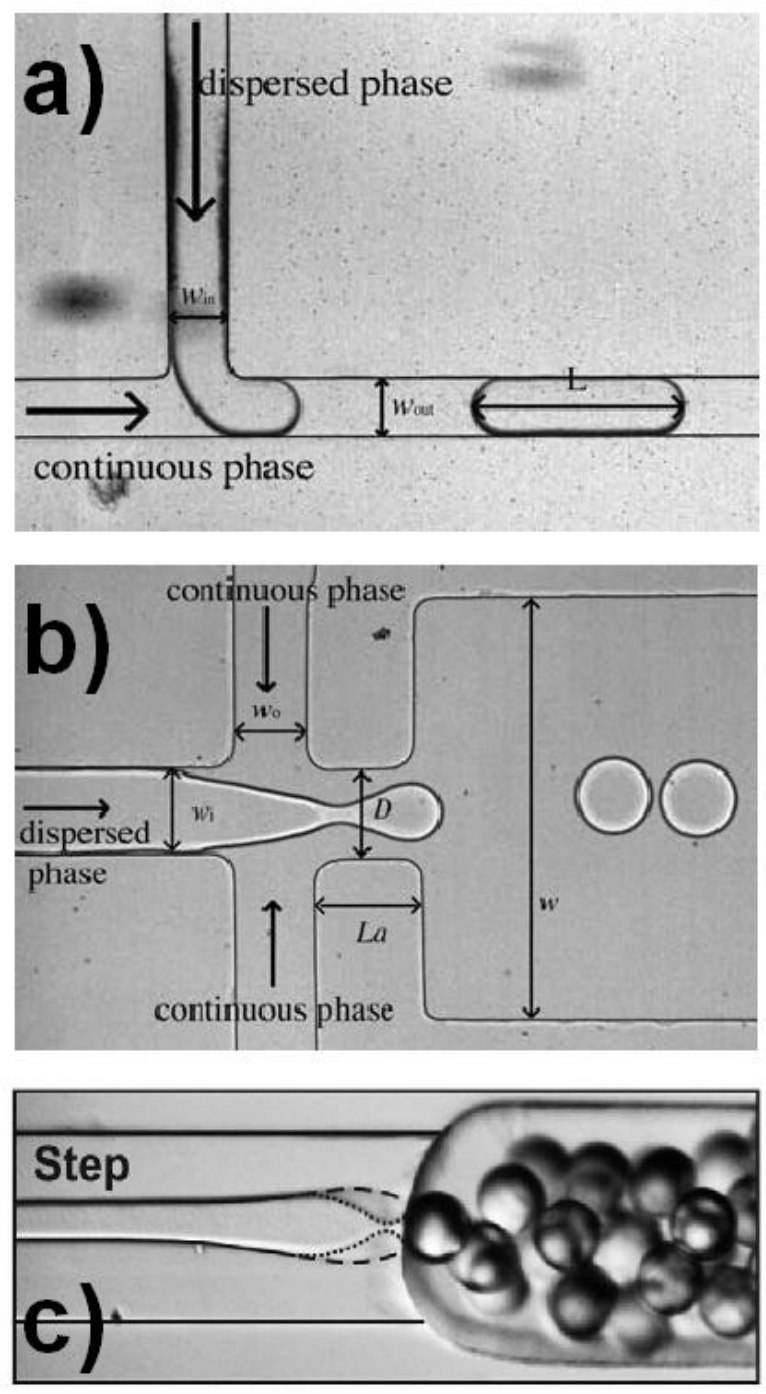

Figure 4.1 Three different type of emulsification implemented on-chip, T-junction (a), and flow focusing junction (b). Reprints from Baroud et al.[41]. (c) Droplet production by step emulsification. Reprint from Priest et al.[42].

The viscous stresses at the interface will lead to the break up of the continuous stream into monodisperse droplets. The presence of a constriction (nozzle) in the exit channel, amplifies the focusing of the phases and allow the reduction of the mean droplet size. See figure 4.1 (b).

Depending on the Capillary number the droplet production exhibits two regimes: the dripping regime where the interface of the elongated part does not move after the separation of the droplet, which can be qualified as "controlled" in the sense that the droplet production is 
regular and the droplet size is highly monodisperse. In this regime the frequency of the droplet production and their volume is easily predictable, see figure 4.1 (b). The frequency of droplet production $f$ depends on the flow rate of the dispersed phases $Q_{\text {dispersed }}$, and on the volume of the droplet $V_{\text {droplet }}$ according to the following law:

$$
f=\frac{Q_{\text {dispersed }}}{V_{\text {droplet }}}
$$

A second regime occurs at higher interfacial stresses where a transition to jetting regime is observed, where the continuous phase elongates to a thin thread. In this regime the thread break-up occurs by the growing of instabilities that makes the size of the droplet unpredictable, and polydisperse. In general the droplet size are of the order of the thread dimension, and can go down to microns.

The last geometry of interest is the step emulsification. This geometry is a variant of the flow focusing junction. See figure 4.1 (c). In this case the jet of the dispersed phase is maintained stable until reaching a step, i.e. a sudden change in the channel height. The sudden change of the flow properties provoke a destabilization of the jet and the break up of the stream in droplets with micrometric sizes. This mode of production is however limited to region where $Q_{\text {dispersed }} / Q_{\text {continuous }} \sim 1$. For a lower ratio the break up will occur at the flow focusing junction as described above. For higher ratio the jet will be stable and no break up will occurs at the step.

To pursue this review, we want to mention two improvements that are brought to the flow focusing junction geometry. First, the emerging field of active control where the emulsion production is modulated by an external field. As an example the droplet production at the flow focusing junction could be modulated by the presence of an electric field, and the development of an additional electrophoretic stress at the interface (Maxwell stress). The application of this technique can induce transition from dripping to jetting regime[44] upon modulation of the electric tension allowing a fast modulation of the frequency production, with transition times of the order of $\sim \operatorname{ms}[45]$. See figure 4.2 .

The second improvement is the flow with an alternating injections of water and oil phases. This technique allows the production of monodisperse multiple emulsions, where the phases are encapsulated one into the other, in the same concept as Matrioshka dolls, see figure 4.3 This technique has been developed in the Weitz Lab [46-48], and requires anisotropic wetting properties of the channel as presented in the figure 4.3 (a). 

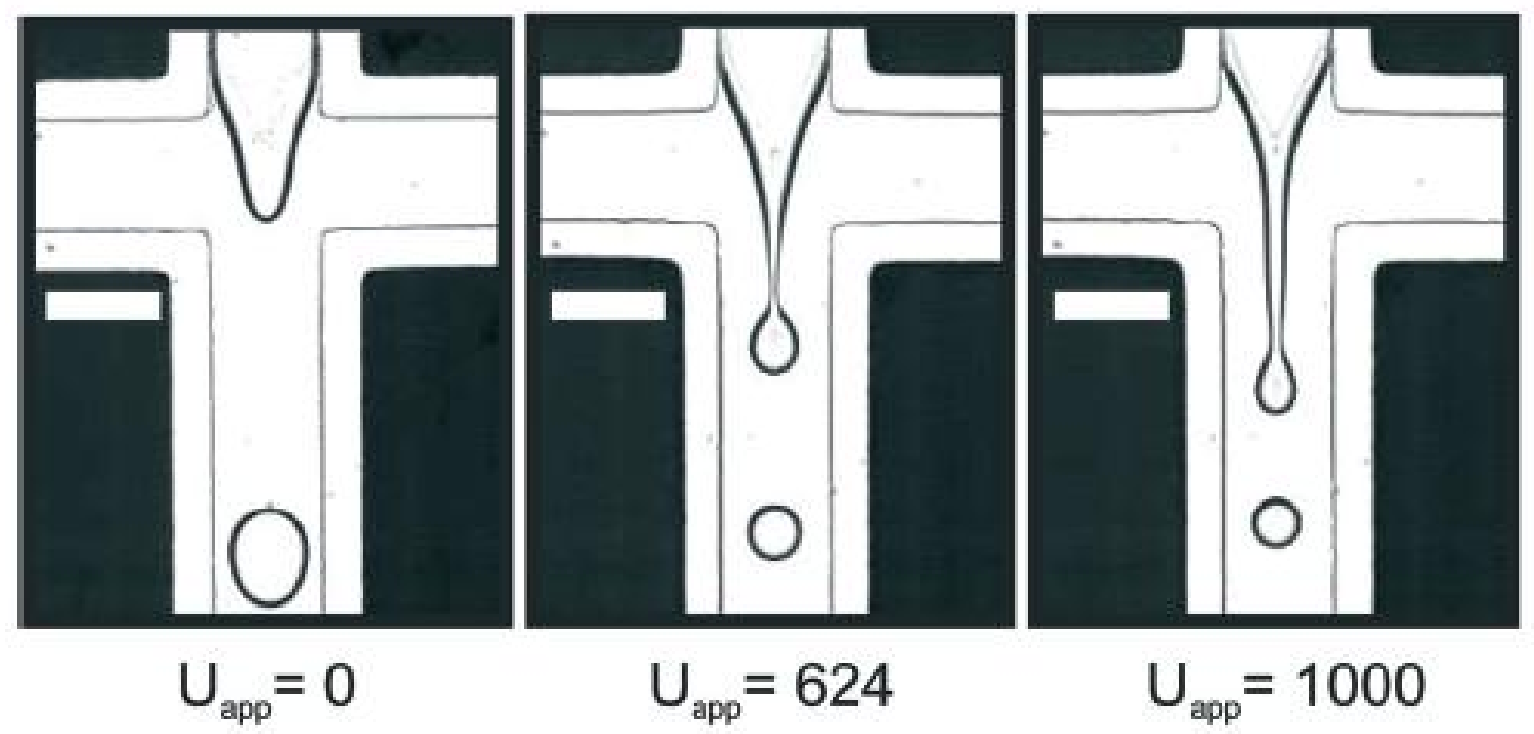

Figure 4.2 Transition from dropping to jetting regime obtained by the modulation of the electric field at droplet flow focusing production $U_{\text {app. }}$. Fluids flow rates is fixed, the electrodes are the black areas on channel sides. Reprint from[44]

\subsubsection{Emulsion aging and methods of stabilization}

In this section we will describe the mechanisms which are provoking the aging of an emulsion via different mechanisms linked to the interfacial merging, and the transport of matter in between droplets. We will describe the solution that can be implemented to prevent the aging mainly by the stabilization of the interface via the formation of adsorbed monolayers, and by the control of the polydispersity.

\subsubsection{Aging}

The micrometric emulsions, given their dimensions present a large surface to volume ratio. The resulting amount of free energy available, renders this macroscopic system metastable and fragile. Therefore a non stabilized emulsion will return progressively to the state of lower energy, that is to say, two homogeneous bulk phases separated by a planar interface. However the mechanisms of emulsion aging are known and can be controlled to ensure a long term stability of the emulsion.

The main aging path is by coalescence which occurs when the thin film between droplets 

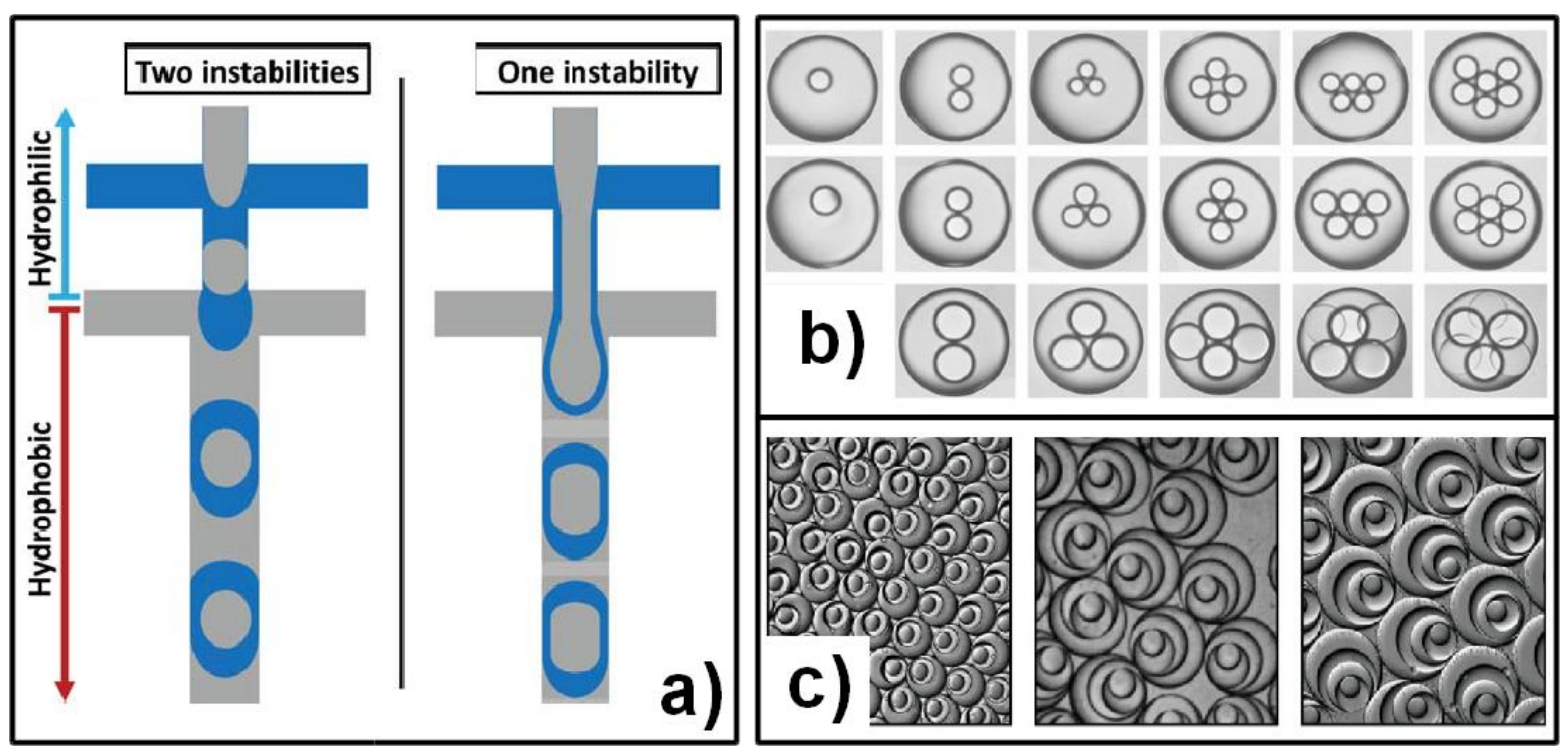

Figure 4.3 (a) Sketch of the typical production of multiple emulsion[49] (b) Double emulsion where the number of encapsulated droplets and their size is varied[47]. (c) Higher order multiple emulsion, obtained by successive dispersion of water phase in fluorocarbon oil. Here triple to quintuple emulsion are displayed. [48]

breaks and the two droplets merge into one entity as depicted in figure 4.4. The precise mechanisms at the interface can be diverse, depending on the presence of surfactant molecules at the interface, or on the presence of an external field.

In general, the emulsion undergoing a dynamic activity, like flowing in microchannels, will be more subjected to coalescence if not stabilized. In this case the growth of instabilities in the thin film separating the droplets will be at the origin of the coalescence[50]. It has been demonstrated in the case of a quick drainage of the interstitial liquid between droplets leads to a local decrease of the pressure. Both droplet interfaces are then pulled closer and merge together [51]. The progression of this disturbance in a train of droplets has been observed. Two droplet merging downstream produce a disturbance and induce a cascade of coalescence propagating upstream[52, 53].

The second main aging mechanism originates from the polydispersity in the droplet sizes that composes an emulsion and is know as the Ostwald ripening. In this case, two droplets in contact with one another will exchange liquid, unless separated by a solid film. The difference of size between two neighboring droplets will induce a different Laplace pressure, that depends of the droplet radius. Therefore a mass transport from the smaller droplet to the larger one 


\section{Chapter 4. On-chip emulsification and hydrodynamics in microfluidic environment}

will occur. Eventually the average size of the droplet composing the emulsion will increase. The production of a monodisperse emulsion, as in microfluidic device, reduce dramatically the aging of the emulsion by Ostwald ripening. In the same way the presence of chemical gradients in the droplet composition can also result is the building of an osmotic pressure at the interface that drives the liquid from the less concentrated droplet to the more concentrated droplet[54].

\subsubsection{Stabilization}

In microfluidics after the droplet production, one needs to ensure the stability of the interface. The production of monodisperse emulsions with the flow focusing geometry is sufficient to cancel the effects coming from the Ostwald ripening. However the circulation of the droplet in microfluidic channel induces viscous stresses at the interface, and the interactions between droplet are ubiquitous. As a consequence, the coalescence is the main effect that needs to be efficiently prevented. An efficient way of stabilization implies the prevention of the contact between the interfaces and/or the maintenance of the liquid film of continuous phase. This is achieved by building a potential barrier at the interface.

We have seen in the previous chapter that the amphiphilic properties of the surfactant molecules adsorb spontaneously to the interface and can form a monolayer at the interface. Here stabilization of interface with the adsorption of surfactant occurs by various processes. The first one is the reduction of the interfacial free energy. We have seen that the presence of surfactant at the interface lowers considerably the surface tension, and therefore the energy gained linked to the interface reduction during coalescence. A second stabilization process arises with the steric repulsion in the case of a water in oil emulsion. This effect is particularly noticeable in the presence of molecules with a long hydrophobic tail. The polymeric entanglement, and loops that spike from the interface to the continuous phase, form a barrier that hinder the contact of two droplets interfaces. Finally, like in the case of micellar aggregates, the chemical potential is high in the entanglement, creating a difference in osmotic pressure, that draws the continuous phases between the droplets. See figure 4.4(c).

Colloidal particle with radii below the micrometer can also spontaneously adsorb at the interface and stabilize an emulsion. The term "Pickering emulsion" has been specifically forged for such materials [56]. The adsorption of solid particles present advantages over the surfactant molecules for the long term stabilization of the emulsions. The particles energy of adsorption 

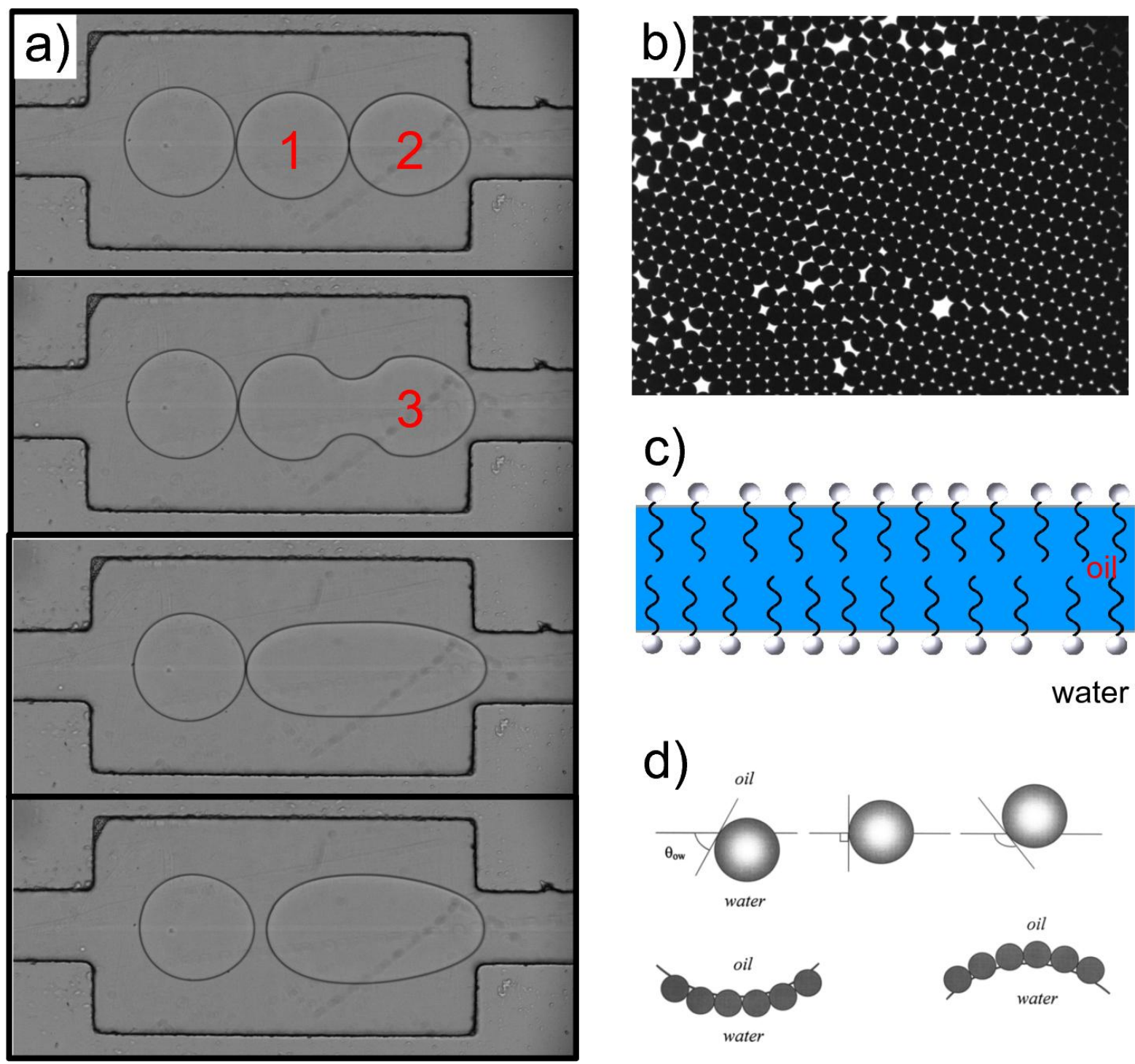

\section{c)}

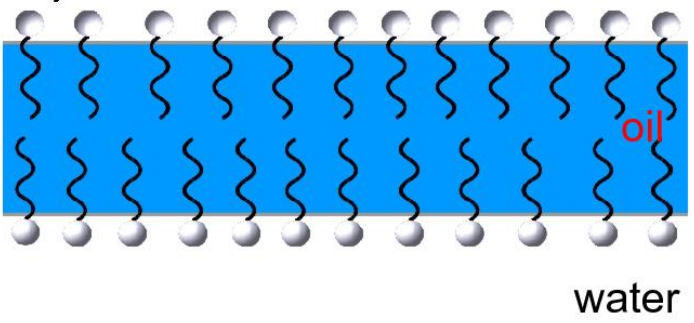

d)
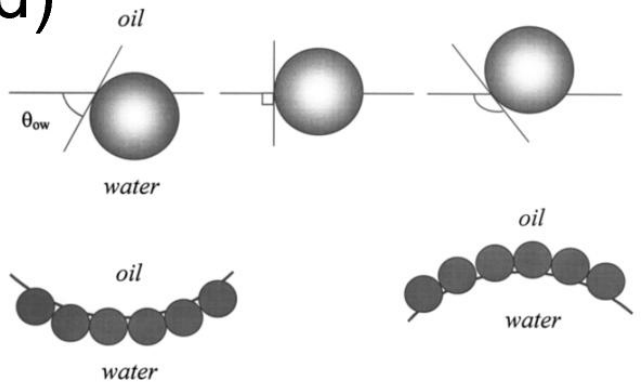

Figure 4.4 (a) The four figure represent an event of coalescence for non stabilized droplet in a microfluidics flow. The interfaces of the droplet marked 1 and 2 enter in contact, and then merge in one single droplet 3 . Eventually the interface under surface tension action returns progressively to an equilibrium shape. The time difference in between two consecutive pictures is $\sim 1 \mathrm{~ms}$ (b) Monodisperse emulsion of a ferrofluid in a water phase, which interface is stabilized by the presence of a surfactant, no coalescence is observed. (c) Idealized picture of an oil/water surfactant stabilized interface. (d) Position of a colloid at a oil/ water interface depending on its contact angle $\theta$ and the resulting bending on the interface. Reprint from [55]. 


\section{Chapter 4. On-chip emulsification and hydrodynamics in microfluidic environment}

is far above $k T$, they are chemically stable, and can prevent efficiently Ostwald ripening.

The stabilization of the emulsion is due to the prevention of the coalescence by the formation of a dense layer at the interface[55]. To give an idea of how solid particles can prevent coalescence, one can consider the reduction of the interfacial area between two partially covered droplets, during the process of coalescence that leads to the compression of the interface and the crystallization of the colloids. At equilibrium, the potential barrier induced by the close packing of the colloids is sufficient to overcome the surface tension forces. The droplets exhibit then, a more or less pronounced prolate shapes, and lay in the state of "arrested coalescence" [57].

The key parameter to characterize the adsorption of solid particles at the interface is the value of the contact angle $\theta$, as it will determine the position of the particle on the interface, and its adsorption energy. See figure 4.4(d). For values of contact angle $\theta<90^{\circ}$, typically inorganic colloids, the water phase is wetting most of the surface of the particle and the curvature imposed to the interface by the packing of the colloids at the interface will favor the encapsulation of the oil phase. In the opposite case, $\theta>90^{\circ}$, usually for organic material, water in oil emulsion are most likely to be formed, as the oil will preferentially wet the colloids[58]. See figure 4.4 (d). Note that the contact angle in this case can be seen as the equivalent for the HLB in the case of surfactants. In both cases the partition of the particle in the different phases is taken into account, and this parameter can serve as a rule of thumb to predict the mode of emulsification.

While adsorbing at the interface the colloids induce an augmentation of the interfacial free energy $G_{\text {colloid }}$ by extending the area of the interface and is described with.

$$
\Delta G_{\text {colloid }}=\pi r^{2} \gamma(1-\cos \theta)
$$

where $r$ is the particle radius, $\gamma$ is the surface tension. This difference in free energy is the cost that the particle has to pay to be adsorbed at the interface. It is dependent on the particle radius $r$, and on its contact angle $\theta$. Therefore particles that are equally wet by both phases, $\theta_{\mathrm{o} / \mathrm{w}}=90$ degree, are more likely to adsorb to the interface and to stabilize the emulsion. Too high or small contact angles will need higher energy to adsorb, and will mainly remain dispersed in the bulk, lowering the stabilization of the interface. 


\subsection{Hydrodynamics in confined flow}

Confined environments such as microfluidic channels are characterized by a strong presence of the boundaries, that have a large influence on the flow profile. In this section we want to describe the flow properties at small dimension in order to understand the flow in microchannels.

In order to describe these flows we will apply the Navier-Stokes equations to a, newtonian fluid. From there we will derive the description of a Poiseuille flow that can be described for laminar regimes, ubiquitous at small dimensions. The adimensionalisation of the NavierStokes equation will allow us to derive the Reynolds number that will be use in the study of inertial effect in the microchannels.

In the second part we will focus on the specificities of a two phases flow, and derive the equation that are relevant to describe the stresses that are taking place at the interface between two non-miscible fluids. We will also give the definition of the Capillary number that is used for the description of interfacial effects.

\subsubsection{The Navier-Stokes equations}

Navier-Stokes equations describes the flow of the fluids as a continuous medium. It establishes the relation between the different properties of the fluid: $\rho$ its density, $\eta$ its dynamic viscosity, $P$ its pressure, $\mathbf{U}$ its the velocity, $[\sigma]$ the tensor of the viscous stress and $\mathbf{f}$ the additional forces that act on the fluid coming from external fields. The Navier-Stokes equations are derived from the the three conservation laws (1) the momentum conservation, (2) the mass conservation, and (3) the energy conservation. In our study the first two one will be necessary to describe our flow conditions [8]. First, the mass conservation gives:

$$
\frac{\partial \rho}{\partial t}+\nabla \cdot(\rho \mathbf{U})=0
$$

and then the momentum conservation results in:

$$
\rho\left[\frac{\partial \mathbf{U}}{\partial t}+(\mathbf{U} \cdot \vec{\nabla}) \mathbf{U}\right]=\rho \mathbf{f}+\vec{\nabla}[\sigma]-\vec{\nabla} P
$$

The term $(\vec{\nabla})$ stands for the gradient operator, and the term $(\nabla \cdot)$ for the divergence operator. 


\subsubsection{Reynolds number}

The adimentionalization of the Navier-Stokes equations, leads to the appearance of nondimensional ratio of the problem parameters[59]. One of this ratio is the Reynolds Number. It is defined as following:

$$
R e=\frac{\rho U L}{\eta}
$$

where $U$ is the velocity of the object of interest, $L$ a typical length that gives the scale of the experiment, $\eta$ is the viscosity of the fluid considered[60]. This ratio is used to compare the relative importance of the inertial effects that scale with $\rho U L$ and the viscous dissipations. The Reynolds number allow the easy distinction of different flow regimes, at different length scales. For the large Reynolds numbers $R e>1$, the viscous dissipations are small in front of the inertial forces. Consequently, the flow will develop turbulent instabilities when encountering obstacles, as presented in the figure. The low Reynolds numbers $R e<1$ characterize the laminar flow regime. In that case the viscous dissipations are important enough to prevent the development of instabilities in the bulk. The resulting flow is characterized by parallel streams lines. In addition the flow at low Reynolds number is reversible in time. The position of a particle of fluid can be tracked back in time is the profile of the flow is known.

\section{Hagen-Poiseuille flow}

The Navier-Stoke equations are non trivial to solve in the general case, but a certain number of simplifications can be made for specific flow conditions. Here we want to give the demonstration of the flow behavior in a straight microfluidic channel with a square section. We consider the flow of an incompressible liquid at low Reynolds number. It is considered as stationary, and unidirectional in the $\mathrm{x}$-direction.

The condition of stationarity and unidirectionality applied to equation 4.4 imposes that the flow is fully developed in x-direction.

$$
\frac{\partial U_{\mathrm{x}}}{\partial x}=0
$$

The conditions given above are also applied in the equation 4.5, and implies the following simplifications. The fact that the flow is stationary cancels all the time dependent terms $\rho \partial \mathbf{U} / \partial t=0$. The unidirectionality of the flow imposes that $U_{\mathrm{y}}=0$ and $U_{\mathrm{z}}=0$. This combined to the equation 4.7 imposes that $(\mathbf{U} \cdot \vec{\nabla}) \mathbf{U}=0$. No external forces are imposed on 
the fluid thus $\rho \mathbf{f}=\mathbf{0}$. The viscous stress can in this conditions be rewritten as $\eta \nabla^{2} \mathbf{U}[8]$, with $\nabla^{2}$ the Laplacian operator. Finally the fluid is considered as incompressible and therefore $\rho$ is a constant and $\nabla \rho=0$.

The resulting equation of the Poiseuille flow in the case of a pressure gradient along the $\mathrm{x}$-axis in the channel writes:

$$
\begin{array}{cc}
\vec{\nabla} P & =\eta \nabla^{2} \mathbf{U} \\
\Delta P & =\eta\left[\frac{\partial^{2} U_{\mathrm{x}}}{\partial y^{2}}+\frac{\partial^{2} U_{\mathrm{x}}}{\partial z^{2}}\right] .
\end{array}
$$

A representation of this flow profile in a channel with a rectangular cross-section is given in figure 4.5.

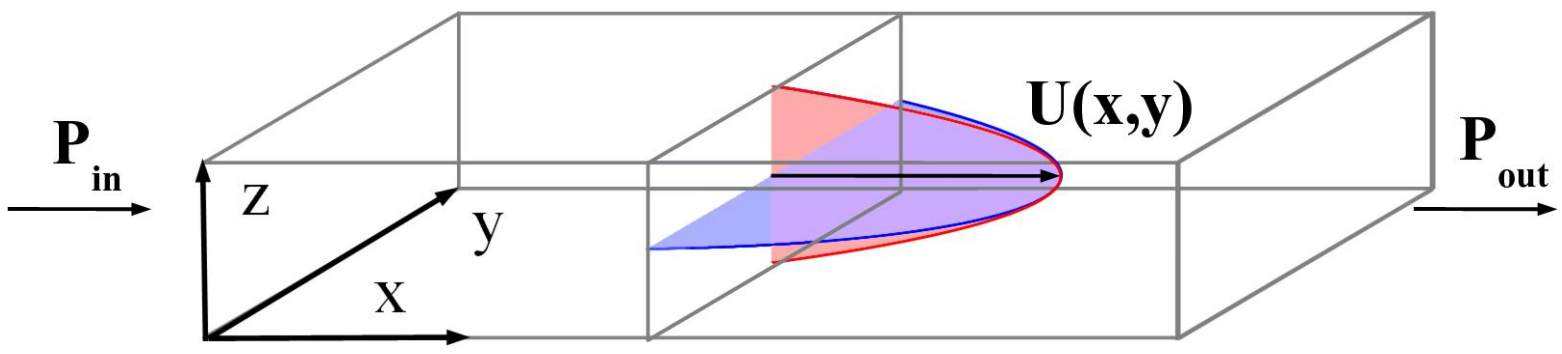

Figure 4.5 (a) Profile of the Hagen-Poiseuille flow in a channel with a rectangular section. The parabolic flow profile in the $y$-direction is represented in blue and the profile in x-direction is represented in red. See equation 4.9. The flow depends on the gradient of pressure $\Delta P=P_{\text {out }}-P_{\text {in }}$

This equation can be solved considering the limit condition at the edges of the channel. The geometry of the channel has a width $W$ and a height $H$, respectively in $y$ and $z$ directions, and for a pressure difference in the $\mathrm{x}$-direction $\Delta P$. In the general case we consider non-slip conditions at the wall that are written:

$$
U_{\mathrm{x}}=0 \quad \text { for } \quad y=z=0 \quad y=W \quad \text { and } \quad z=H
$$

The resolution of the Stokes equation (equation 4.9p results in the expression:

$$
Q=C \frac{H^{3} W \Delta P}{L \eta}
$$

where $Q$ is the flow rate of the liquid, $\Delta P$ the pressure drop along the channel $C \sim(W / H)$ 


\section{Chapter 4. On-chip emulsification and hydrodynamics in microfluidic environment}

is a friction coefficient that takes into account the aspect ratio of the channel. $L$ is the length of the considered channel. $C$ takes values between $0.04-0.08$ for channels aspect ratio $(W / H)$ between 1-10[18].

\subsubsection{Interfacial limit conditions}

For a static system the continuity equation at the interface is decomposed in a normal and tangential component. The general stress balance at the interface is given by the continuity of the normal and tangential component:

$$
\mathbf{n} \cdot[\sigma]_{\mathrm{c}}-\mathbf{n} \cdot[\sigma]_{\mathrm{i}}=\gamma \mathbf{n}(\nabla \cdot \mathbf{n})-\nabla \gamma
$$

where $[\sigma]_{(\mathrm{x})}$ is the tangential stress tensor, and $\mathbf{n}$ is the unit vector normal to the interface pointing from the inside (i) to the outside (c) and $\gamma$ the surface tension. See figure 4.6

The continuity equation (equation 4.12 projected on the vector normal to the interface gives:

$$
\mathbf{n} \cdot[\sigma]_{\mathrm{c}} \cdot \mathbf{n}-\mathbf{n} \cdot[\sigma]_{\mathrm{i}} \cdot \mathbf{n}=\gamma(\nabla \cdot \mathbf{n})
$$

where the quantity $\nabla \cdot \mathbf{n}$ represents the divergence of the local curvature. Therefore changes in the normal interfacial stress are modifying the local curvature, and these changes are proportional to the surface tension. This equation represents the deformability of the interface upon external force fields In static case where only the pressure force is present, the Laplace equation is recovered. The projection of the the continuity equation on a vector normal to the interface $\mathbf{t}$, is written as:

$$
\left([\sigma]_{\mathrm{c}} \cdot \mathbf{n}\right) \cdot \mathbf{t}-\left([\sigma]_{\mathrm{i}} \cdot \mathbf{n}\right) \cdot \mathbf{t}=\nabla \gamma \cdot \mathbf{t}
$$

The right hand side of this equation is particularly interesting as it leads to the conclusion that a static system can only be achieved if no surface tension gradients are present at the interface. Lets consider an element of the interface as represented in figure 4.6, small enough to be considered flat, with a gradient of surface tension is the $\mathrm{x}$-direction. The gradient can be expressed linearly by $\Delta \gamma=\gamma_{1}-\gamma_{2} \neq 0$. The local stress gradient is given by the shear on the interface, proportional to the velocity gradient $\partial U_{\mathrm{x}} / \partial y$ in the neighborhood to the interface and the viscosity $\eta$. The tangential stress balance condition becomes[8]:

$$
\eta_{\mathrm{i}} \frac{\partial U_{\mathrm{x}}^{\mathrm{i}}}{\partial y}-\eta_{\mathrm{c}} \frac{\partial U_{\mathrm{x}}^{\mathrm{c}}}{\partial y}=\Delta \gamma
$$




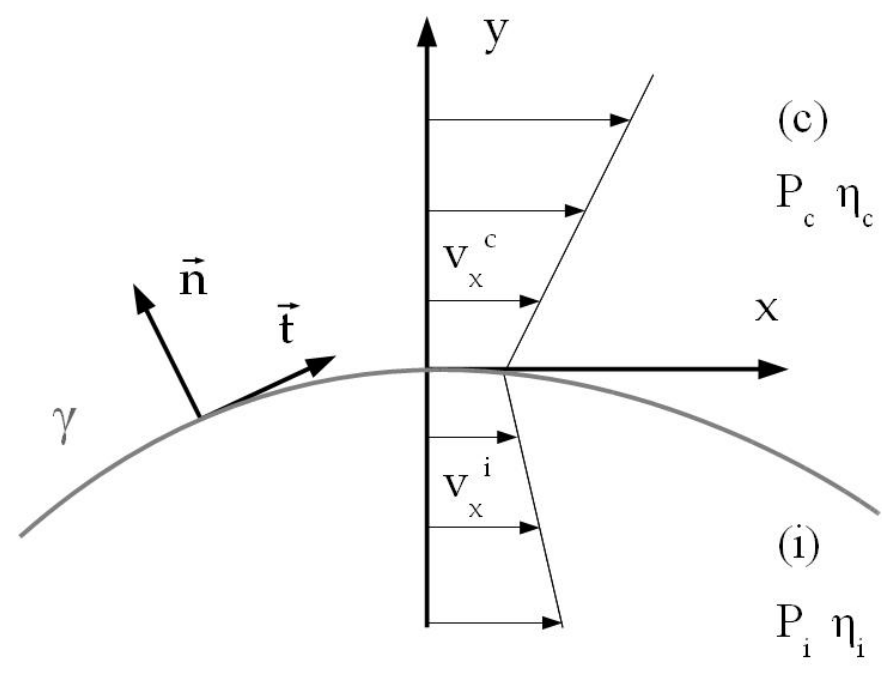

Figure 4.6 Schematic view of the continuity equation at one interface. The domain (i) represent the inside of the droplet, while domain (c) represents the continuous phase. Each phase is characterized by its viscosity $\eta$ and the pressure $P$. The interface is materialized by a gray line is defined by the surface tension $\gamma$.

A correlate to this conclusion is that an interface at rest with a surface tension inhomogeneity will produce a flow in the phases near the interface. These types of flow are called Marangoni flows. The direction of the flow depends on the sign of $\Delta \gamma$, going from the region of low surface tension to the region of high surface tension. The velocity of the flow is proportional to the magnitude of the gradient.

A small energy is required to displace surfactant molecules at the interface and consequently produce surface tension gradients. As a consequence Marangoni effects are closely linked to the presence of an adsorbed surfactant layer at the interface. In this case the Marangoni flows act as a recovering effect that tends to re-homogenize the distribution of the surfactant. Marangoni forces are one contribution to the development of an interfacial viscoelasticity for the deformation of a surfactant-laden interface[61].

\section{The Capillary number}

We have seen from the equation 4.15 that the viscous stresses induced by the hydrodynamic flows at an interface scale as $\eta U$. On the other side the interfacial forces coming from the Young-Laplace equation are scaling as $\gamma$. The ratio of these forces defines the Capillary num- 
ber

$$
C a=\frac{\eta U}{\gamma}
$$

This non-dimensional ratio is used to compare the relative forces coming from the fluid that promote the interface deformation and the interfacial tension that opposes to the deformation. Therefore for a large Capillary number $C a>>1$, the force acting on the interface will largely overcome the surface tension and provoke a large deformation of the interface up to the break up of the interface. For $C a<<1$ the viscous stresses are too small in front of the surface tension, and a small deformation of the interface will be visible. The extreme case of a non deformable interface (solid) can be described with the asymptotic condition of $\mathrm{Ca} \rightarrow 0$.

This chapter has presented the background necessary to understand the mechanisms of on-chip emulsification that will be used in our study. In addition, the basic knowledge to understand a describe the interface deformation in confined environment were also given. This chapter also pointed out the necessity to circumvent the coalescence in channel and the method to stabilize on-chip emulsions. It has been shown that the presence of a surfactant adsorbed layer is usually sufficient to solve this problem. In the coming part we will present the different studies implemented for the characterization of the adsorption kinetics of perfluorosurfactants. 


\section{5 Experimental methods}

The present chapter is a review of the experimental methods used in this work. In a first part we will briefly recall the principles of already well-established methods like the pendant drop tensiometry, and dynamic light scattering (DLS). In a second part we will present the methods which have been developed in our lab for the purpose of this work.

\subsection{Surface tension measurements: Pendant drop method}

The pendant drop measurements are made using a commercial apparatus (PAT-1M SINTERFACE). The droplet is produced at the tip of a needle of known outer diameter $d_{\text {out }}=0.5-1 \mathrm{~mm}$. The droplet area, volume and contour is monitored in real time ( 25 frames per second) by a camera (figure 5.1 (a) A) situated on the same horizontal plane of the drop in its measurement chamber (figure 5.1 (a) B). The volume of the droplet is kept constant by a retro-control acting on the actuated glass syringe (figure 5.1 (a) C), that dispenses / refills the amount of liquid needed. The surface tension is extracted from each frame through the analysis of the droplet contour described in the chapter 2. The equilibration time extends from $10^{2}$ to $10^{5}$ seconds, depending on the nature of the surfactant, and the concentration used. We perform the measurement of surface tension between pure oil and water. 

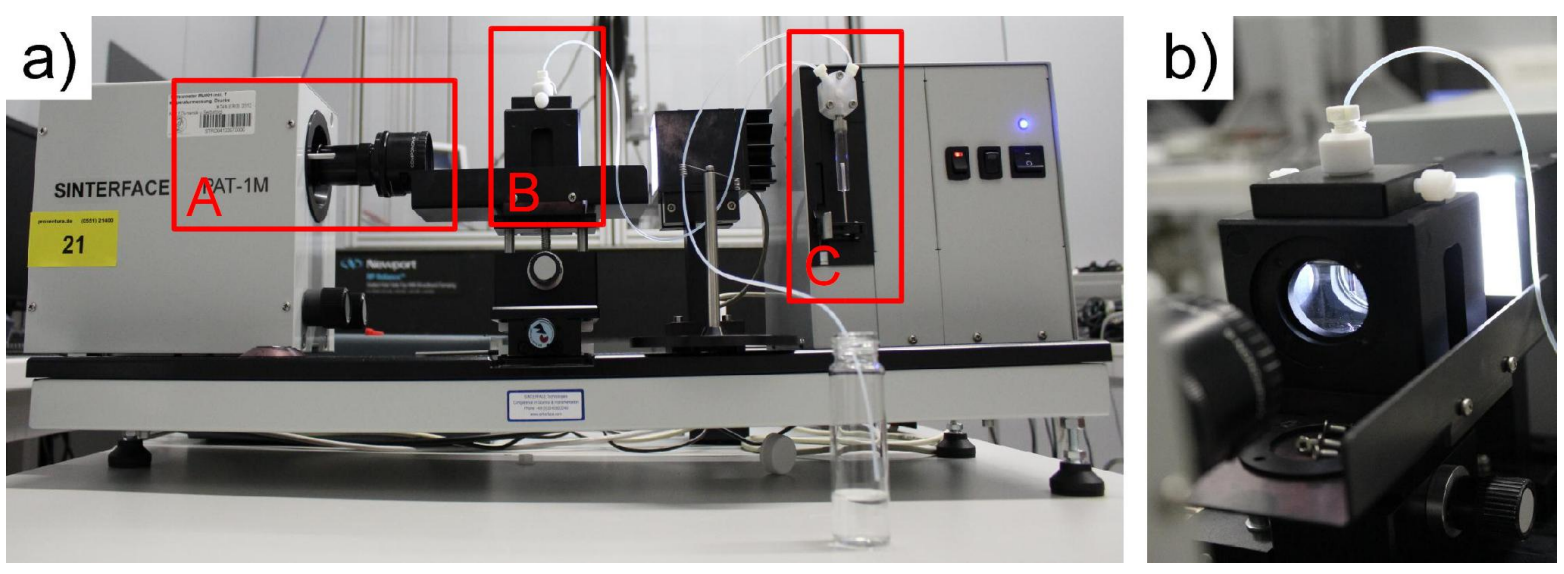

Figure 5.1 (a) General aspect of the PAT-1M SINTERFACE apparatus. (b) Close up on the measurement chamber where the droplet is visible. An example of the numerical treatment associated to the detection of the interface is presented in chapter 2

\subsection{Dynamic light scattering}

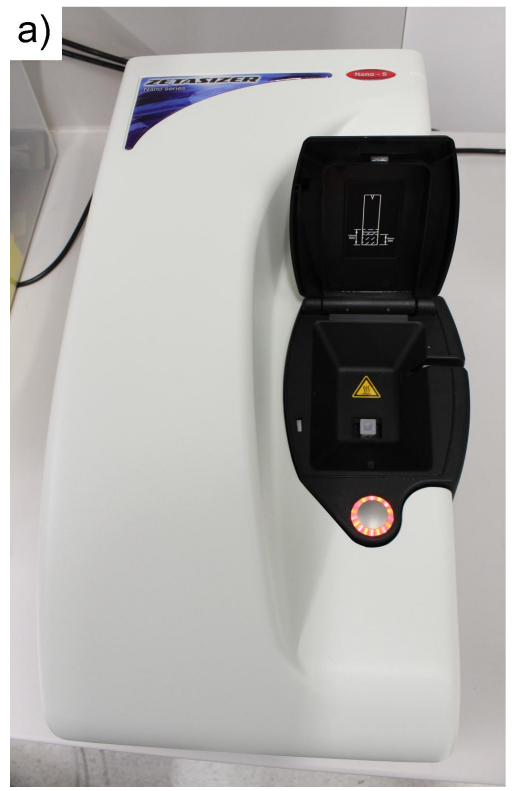

b)

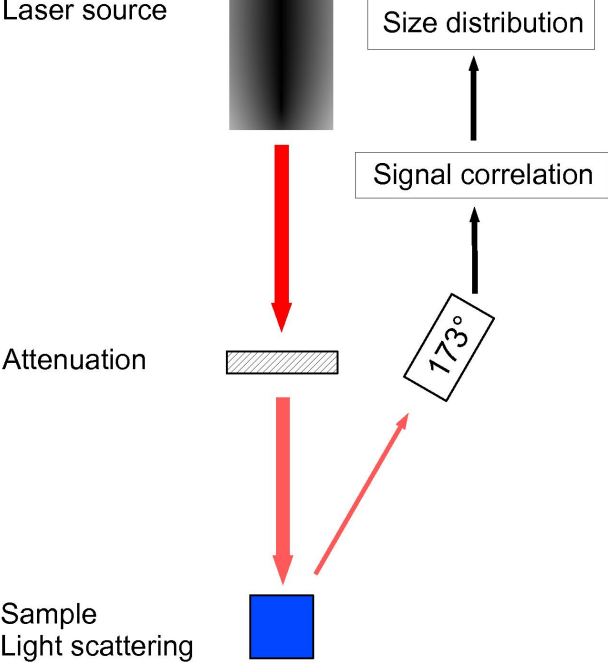

Figure 5.2 Dynamic light scattering technique. (a) Picture of the apparatus, (b) principle of the DLS measurements.

The dynamics light scattering technique is used for the quantitative measurements of the number of particles present in solution as well as the size distribution. The method is based 
on the Rayleigh scattering of a monochromatic light beam with wavelength $\lambda_{\mathrm{s}}$ on dispersed particles in the colloidal range. In our case the technique will be applied to measure micelles size and population, and the determination of the $\mathrm{CMC}[$ [62]. We use the apparatus Zetasizer Nano S (Malvern) depicted on figure 6.12 (a).

The figure 6.12 (b) presents a schematic of the apparatus. A monochromatic beam is produced by a Laser source and directed to the sample. The beam intensity can be modulated by a attenuator, and the measurements site moved by a movable lens. We use transparent disposable PMMA cuvettes (Fisher) as container for our sample. Here we collect the backscattered light at an angle of 173 degree, this angle allows the measurement on the edge of the cell, preventing the disturbance of the signal by multiple scattering events, and dust detection. The scattering intensity signal is then post-processed in order to obtain the time-dependent autocorrelation function. The signal is then processed with the Stokes-Einstein relation in order to extract the size distribution of the sample. The calibration of the apparatus has shown a good sensitivity for dilute sample in the range of particles with a dimension of $5-500 \mathrm{~nm}$.

\subsection{Microfabrication}

The microfluidic chips are designed following the classical soft-lithography methods. We use microfluidic chips made of a liquid adhesive, Norland Optical Adhesive (NOA), crosslinking under U.V. The reason for this choice is based on the good mechanical properties of the material, especially its Young modulus, and its chemical resistance. The pressure drop that builds along a microfluidic channel with a rectangular cross-section depends strongly on the channel height (equation 4.11). Therefore the use of microchannels with heights of the order of $100 \mu \mathrm{m}$, and delay line of the order of few centimeters, can result in a pressure drop that are of the order of a few bar. The PDMS Young modulus, $600 \mathrm{kPa}$ is in the same order as the pressure drop. As a result a clear deformation of the walls occurs during the experiment. This kind of deformations have already been noticed, and even advantageously used for a high parallelization of droplet production by Dangla et al. [63-65]. In appendix A we show two experiments methods that where implemented to quantifying the PDMS channel deformations under flow conditions.

\subsubsection{Clean Room techniques}

The roman numerals used in the description below are as in the figure 5.3 . 


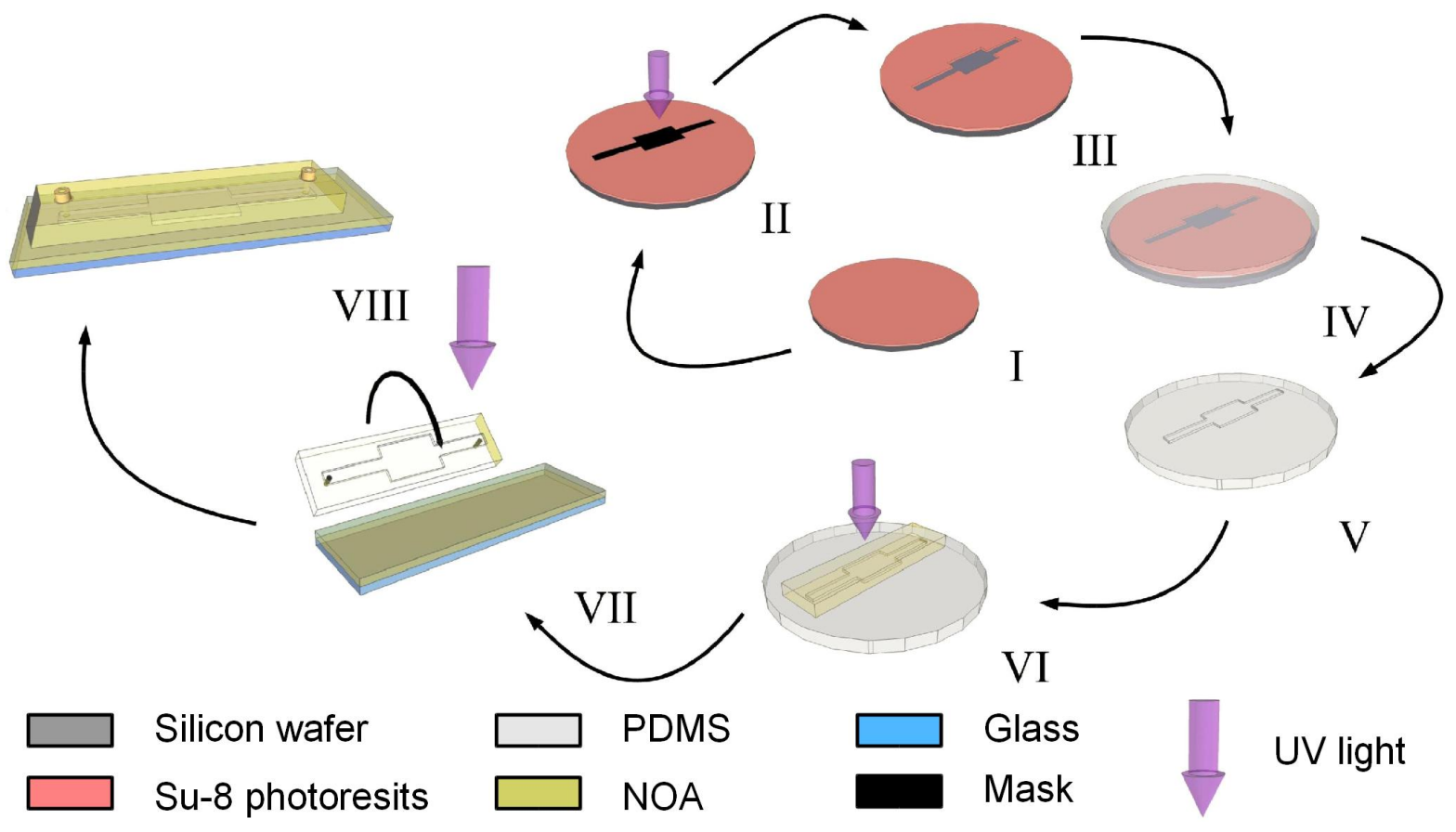

Figure 5.3 Detailed schematic for the different steps of microfabrication of the NOA chips used in our experiments.

The aim of the clean room work is to obtain a mold made of epoxy resin SU-8 of controlled thickness, that will be used to create replica by casting of liquid NOA. We start with a flat Silicon wafer as a base for our mold.

(I) A layer of SU-8 resin is spincoated in order to achieve a homogeneous repartition of the photoresist resin on the surface. The thickness of the layer is determined by the choice of the photoresist in a range of a given viscosities and the rotation speeds according to preestablished tables.

(II) The photoresist goes through a step of soft-baking a first time at $95{ }^{\circ} \mathrm{C}$ during $20 \mathrm{~min}$ in order to start the evaporation of the solvent and obtain a solid surface.

(III) A mask is brought in soft contact with the SU-8 surface. We use a negative photoresist, where the polymerization is enabled by the exposure to U.V.

(VI) The resin goes through a post-exposure bake step, with a $10 \mathrm{~min}$ ramp from $65^{\circ} \mathrm{C}$ to $95^{\circ} \mathrm{C}$ and then down to $65^{\circ} \mathrm{C}$ for additional $10 \mathrm{~min}$. This step allows the final evaporation of the solvent as well as the polymerisation of the exposed photoresist. A ramp is recommended to avoid the apparition of cracks during the polymeration resulting from temperature gradients within the layer. 
(V) The non cross-linked parts of the SU-8 layer are dissolved with a developer. To obtain a NOA chip, a negative SU-8 structure is needed, therefore the geometry of the channel is protected from the UV beam. As a result we obtain the so-called negative of the geometry. The non cross linked photoresist is washed away, and the thickness of the remaining SU-8 layer is measured via interferometry.

\subsubsection{Fabrication of the NOA chips}

The following part is not performed in the clean room but recommended to be done in a dust controlled area, to avoid the dust particles embedding in the channel.

(VI) From the negative SU-8 mold, we cast a positive mold made of Polydimethylsiloxane (PDMS) by pouring in a liquid solution of PDMS and cross-linker (concentration 10\%). One hour in oven at $75^{\circ} \mathrm{C}$, is sufficient to obtain a solid PDMS-positive.

(VII) The NOA structure is obtained by casting of liquid NOA on the PDMS-positive. One essential property of this reaction is the prevention of the cross-linking reaction by the presence of oxygen[64]. Therefore upon exposure, a layer of mere cross-linked material will exist at all surfaces in direct contact with air and on surfaces in contact with PDMS porous to oxygen.

(VIII) We prepare the channel structure by casting of NOA on PDMS mold and by exposure to U.V. during 20 seconds. A NOA substrate, which consists in a thin layer of NOA on a glass plate is prepared separately in the same way. Both parts are finally brought in contact and exposed to UV for $20 \mathrm{~min}$, to ensure the adhesion of both parts.

To finalize the microfluidic chip, connectors are glued at inlets and outlets of the chips, and the channel are hydrophibized with by the coating of the walls with an Aquapel solution.

\subsection{Microfluidics}

Now we present the experimental setup used in the study of the droplet deformation and in the measurement of the surface tension.

\subsubsection{Fluid control}

In order to produce monodisperse emulsions one needs a reliable control of fluid motion. A constant flow rate is maintained in our channel from glass syringes (Hamilton, $5 \mathrm{~mL}$ ) using 


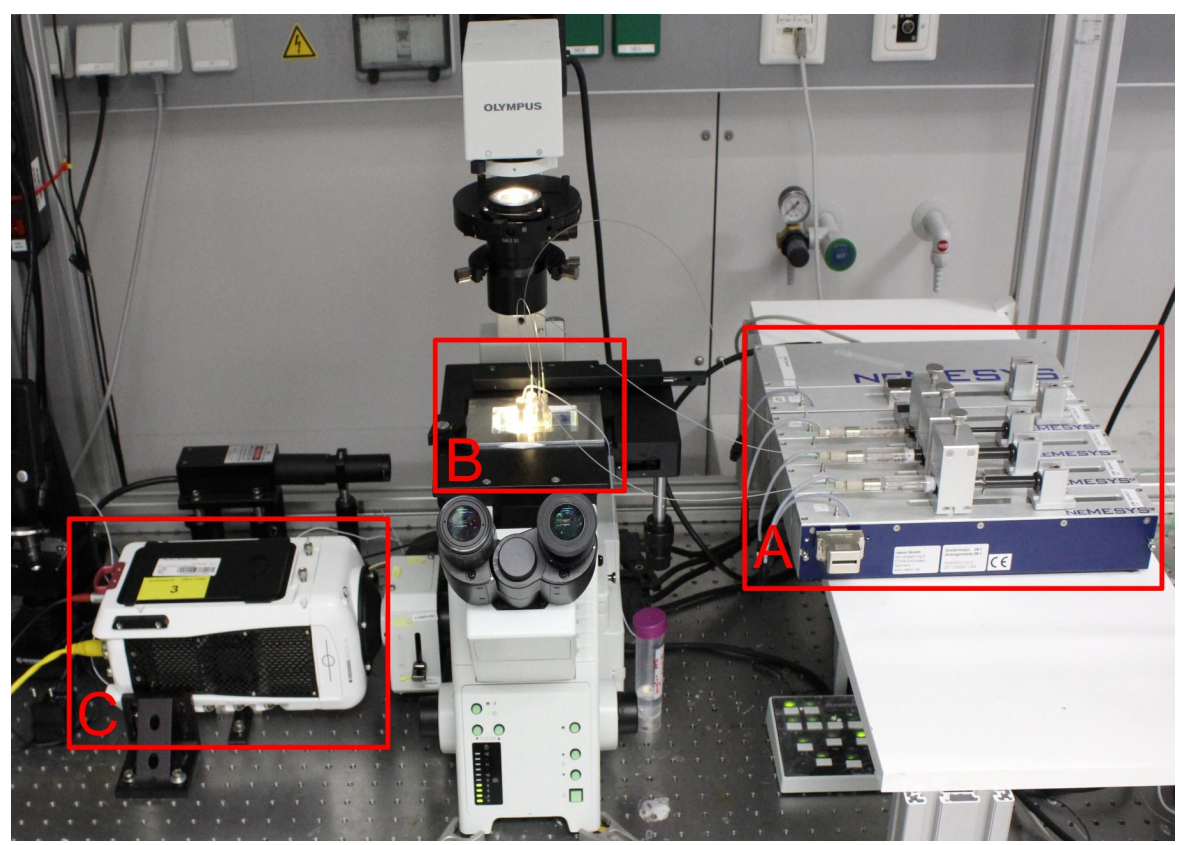

Figure 5.4 Microfluidic experimental setup for the measurement of the droplet deformation. The liquid phases are driven from the syringes by the syringes pumps A to the microfluidic chip B. The detection of the droplet shape during their travel through the microfluidic channel is made with a high-speed camera $\mathrm{C}$ thought a microscopic device.

syringe pumps (Nemesys, Cetoni figure 5.4 A). The precision of the flow rate, is ensured by a calibration beforehand of the syringe pistons pushers translation speed, based on the syringe diameter. The syringes and microfluidic chip are connected via a PEEK tubing of outer diameter $0.75 \mathrm{~mm}$.

\subsubsection{High-Speed Microscopy}

The chip is mounted on an inverted microscope (Olympus IX71 figure 5.4 B) and imaged using a X20 microscope objective. The pictures of the droplets are recorded with a high speed camera (Phantom, V311 figure 5.4 C) at a frame rate of 4000 to $10000 \mathrm{fps}$, and exposure time of $1 \mu \mathrm{s}$. The movies of droplet flow are recorded in each of the deformation chamber or in a subset of those. A 20X magnification is used for the recording. This magnification allow a sufficient resolution of the droplet interface and a field of view large enough to monitor the droplet deformation in the channel. Picture are presented in figure 5.5 (a) and (e). 


\subsection{Image processing and data extraction}

The videos are processed with a home-developed routine, with the goal of extracting the geometrical conformation of the droplet from each frame. An example of this processing is shown in figure 5.5

The scale of the image is given by the user. Four reference points set in the corners of the expansion, are used to define a conversion rate between the number of pixels in SI units. It defines as well the orientation of the $x$ and $y$ axis respectively parallel and perpendicular to the channel walls. From the diagonals linking the opposite corners the coordinates of the center of the expansion $x_{0}$ and $y_{0}$ is extracted and used to define the relative position of the droplet, see figure 5.5 (i).

In figure 5.5 we show the different steps of the image processing for a droplet which contour is well defined (figure 5.5 (a)), in opposition to a droplet contour that merged with the channel wall (figure 5.5 (e)). The image treatment is implemented as follow. For each frames of the video an average image of the background is produced (figure 5.5 (i)), then subtracted from the original image. The contour of the droplet is detected from the remaining foreground figure 5.5 (b) and (f). When the droplet contour is close to the channel wall, a part of the contour is lost during the subtracting of the average picture. See the transition from figure 5.5 (e) to (f). The necessity to obtain a close contour forces us to recover this part. It is possible to merge domains close to each other with the "blob" function. This is visible between figures 5.5 (f) to $(\mathrm{g})$. When the separated domains are too far apart from each other, the contour is detected by the implementation of the function "Convex Hull" [66] that draws the minimal convex line including all the points detected as being part of the droplet contour. We can see it as an elastic band that closely mold the contour of the droplet. The missing parts are with this method assumed to be straight lines. We manage to recover the missing parts efficiently when the droplet circulates through the channel(figure 5.5 (f)) to (g), where the contour indistinguishable from the (straight) channel wall and disappears in the removing of the background. The main limitation however, the poor detection of concave surfaces, that will also be approximated by a straight lines. We choose to keep this approximation given the rare events of concave interfaces in our experiments.

The droplet is now described by a domain comprised in the Hull geometry. The droplet area $A$ is calculated by converting the number of pixels in this domain to SI units. The center of mass coordinates $\{x, y\}$ are calculated by averaging of the coordinates of the $n$ pixels of the 
domain $x_{\mathrm{i}}$ and $y_{\mathrm{i}}$ :

$$
x=\sum_{\mathrm{i}}^{\mathrm{n}} \frac{x_{\mathrm{i}}}{n} \quad \text { and } \quad \mathrm{y}=\sum_{\mathrm{i}}^{\mathrm{n}} \frac{\mathrm{y}_{\mathrm{i}}}{\mathrm{n}}
$$

Given the moderate deformation observed on the chip, a droplet contour is assumed to be an ellipse. We define the axis of the ellipsoid $l$ and $L$, by the maximum length limited by the contour in $x$ and $y$ direction, passing thought the coordinates $\{x, y\}$ of the droplet center of mass. The perimeter $p$ is calculated from the number of pixels forming the contour and converted to SI units.

The instantaneous velocity $\left\{U_{\mathrm{x}}, U_{\mathrm{y}}\right\}$ is extracted by division of the difference in position of the center between two frame $i$ and $i+1$ by the time between two frames $t_{\mathrm{f}}$ :

$$
U_{\mathrm{x}}=\frac{x(i+1)-x(i)}{t_{\mathrm{f}}} \quad \text { and } \quad \mathrm{U}_{\mathrm{y}}=\frac{\mathrm{y}(\mathrm{i}+1)-\mathrm{y}(\mathrm{i})}{\mathrm{t}_{\mathrm{f}}}
$$

We have access now to the deformation, area, and speed profiles of the droplet thought the expansion geometry. The profile is in fact constituted by the superposition of all the profiles of all the $N$ droplets flowing through the channel during one recording. $N$ varies depending on the droplet production conditions, and on the time of recording. Each experimental conditions presented afterwards will detail the typical number of droplets detected.

\subsubsection{Comments on the method}

The main limitation that originates from the "counting of pixel method", is the discretization of the values. It can lead to important deviations in the calculation of the perimeter, especially for objects that are of the order of $\sim 10$ pixels in diameter. In our case, due to the important magnification, the projected area of a droplet is of the order of $A \sim \times 10^{4}$ pixels, so the lengths are of the order of $\sim 300 \times 10^{1}$ pixels which is enough for a good resolution. More important the relative deformation of these axis during a deformation can be extremely small, especially for high surface tensions. We see that here a deformation of $1 \%$ in the length would correspond to a change of 3 pixels. These are detectable but highly discretized.

An efficient way to improve this method would be the implementation of an analytical fitting of the interface, based on spherical harmonics. An interface function would offer a continuous description, easy to compare to numerical models of deformations. 

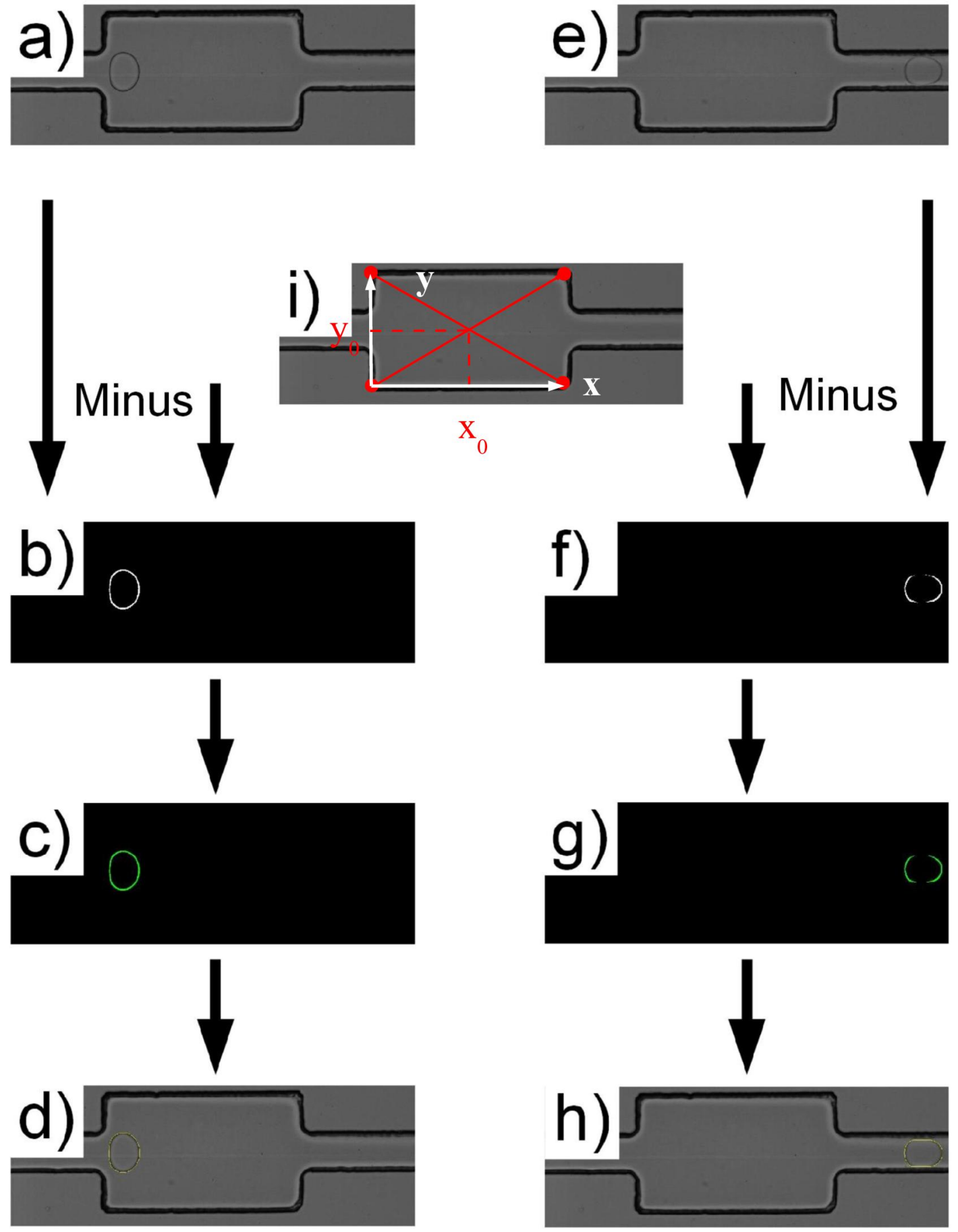

Figure 5.5 Different steps of the droplet contour extraction for a droplet at its maximum deformation (a) to (d) and for the droplet confined in the constriction (e) to (h). We have in order: the original picture (a) and (b); the foreground picture (b) and (f); the aggregated contour (c) and (g); and the Hull contour (d) and (h). The picture (i) is the averaged picture. 



\section{Characterization of perfluorinated surfactants}

Parts of this work have been published in the article "Microfluidic Dynamic Interfacial Tensiometry: $\mu$ DIT”, Soft Matter, 2014

\subsection{Introduction}

The applications that use droplets as a microreactor have increased dramatically with the recent development of the droplet-based microfluidics. In these techniques, the stabilization of the droplet interfaces relies mainly on the use of surface active molecules. Among these applications, the droplet has recently become a means for the study of biological material, and more particularly the encapsulation of living material, cells or bacteria, in micrometric droplets [39, 67].

Usually, such systems rely on the delivery of the nutrients necessary for the development of the organisms, and on the efficient exchange of the oxygen and carbon dioxide between the droplet and the surrounding medium. This last feature was demonstrated to be possible by the use of some fluorinated oils with excellent properties of oxygen and carbon dioxide solubilization. An obvious condition that also determines the success of such applications is 

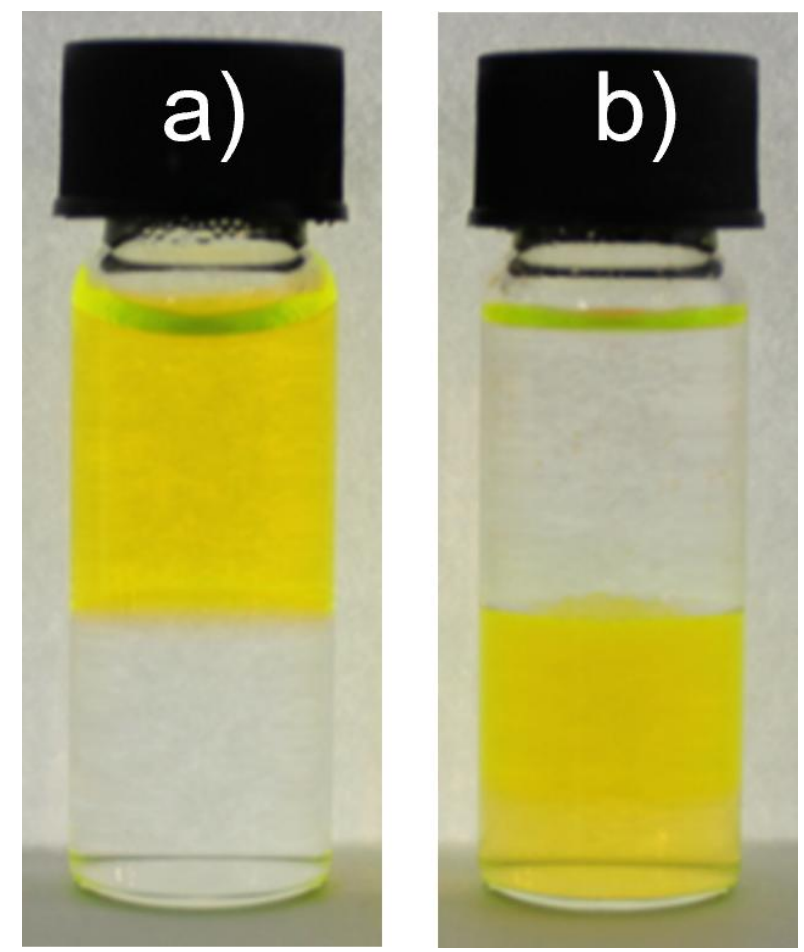

Figure 6.1 Changes of partition of a fluorescent dye (Here Rhodamine $100 \mu \mathrm{mol} \cdot \mathrm{L}^{-1}$ ) in presence of Krytox-H surfactant. (a) The fluorescent dye is in solution in the water phase (top phase), and do not go in the interface (bottom phase). (b) In presence of surfactant, the dye is becoming more soluble in the oil phase. This is an example of how the presence of a perfluorosurfactant molecule can change the transport properties at the interface. Courtesy Philipp Gruner.

the biocompatibility of the droplet environment. The biological materials are particularly sensitive to slight variations of the $\mathrm{pH}$, and charges in their environment. [68] This is particularly true in the condition of droplet volumes $\sim \mathrm{pL}$ used in microfluidics, where the presence of even small amount of contaminants is sufficient to change the properties of the medium. See figure 6.1 .

The choice of the surfactant that stabilizes water in perfluorinated oil emulsion is then limited by the constraints detailed above. Among the surfactants that are soluble in the perfluorinated oil, the perfluoropolyehter (PFPE) based molecules present a chemical reactivity that make them fairly customizable by well established chemical syntheses [69, 70]. As an example, a macromolecules with chemical structure based on the of PFPE chain have been proved to conveniently stabilize emulsion, without significantly changing the properties of the transport through the droplet interface[71]. 
To date, among the works focused on the description of the surface active properties of these new fluorinated molecules, only few dynamic tensiometry characterization have been performed[72]. These studies are mainly limited to the general comparison of the dynamic surface activity and the link to the physical-chemical properties of the molecules. We propose that a precise study of the surface active properties, as well as the kinetic properties of simple PFPE chain would help us to predict the general interfacial behavior of their derived macromolecule.

In this chapter we present the first in-depth characterization of three perfuorosurfactants of the same family, of importance for industrial and biological applications. We present the study of two simple anionic PFPE chains that only differ by the neutralizing counter ion. In addition we will compare their adsorption kinetic properties to the properties of a triblockcopolymer macromolecule chemically synthesized from these molecule. This analysis is also used to choose the best reference system, and to constitute a reference set of the molecules kinetic properties that can be compared to the on-chip dynamic tensiometry measurement that is developed in the following chapter. We use the classical pendant drop method to access the equilibrium surface tensions and extract the properties of the dynamic equilibrium of the interface. In addition, we will discuss the dynamics surface tension profile. We will see that diffusive processes are mostly dominating the early kinetics, but that the late kinetics can be subject to transfer limited regimes under some experimental conditions.

The discussion will be divided as follow. In the first part we will describe the chemical structure of the three molecules, and the possible effects upon adsorption at the interface. In the second part we describe the method used for the tensiometry measurements. Later the analysis of the three surfactants will be divided in two parts: one dedicated to PEG-PFPE molecule which is a neutral surfactant. The latest will be focused on both charged molecules Krytox-H and Krytox- $\mathrm{NH}_{4}$. The last part will be dedicated to characterization of the micellar aggregates, that are found above the CMC, by using dynamics light scattering. We want to acknowledge Dr. Estelle Mayot from ISIS, and Dr. Brian Hutchinson from the Raindance Technologies company for the kind gift of the surfactant molecules used throughout this study.

\subsection{Molecular structures}

The chemical structure of our three surfactants are shown on figure 6.2. Krytox-H and Krytox$\mathrm{NH}_{4}$ are two perfluoropolyether (PFPE) chain with approximatively 13 units of repetition, 

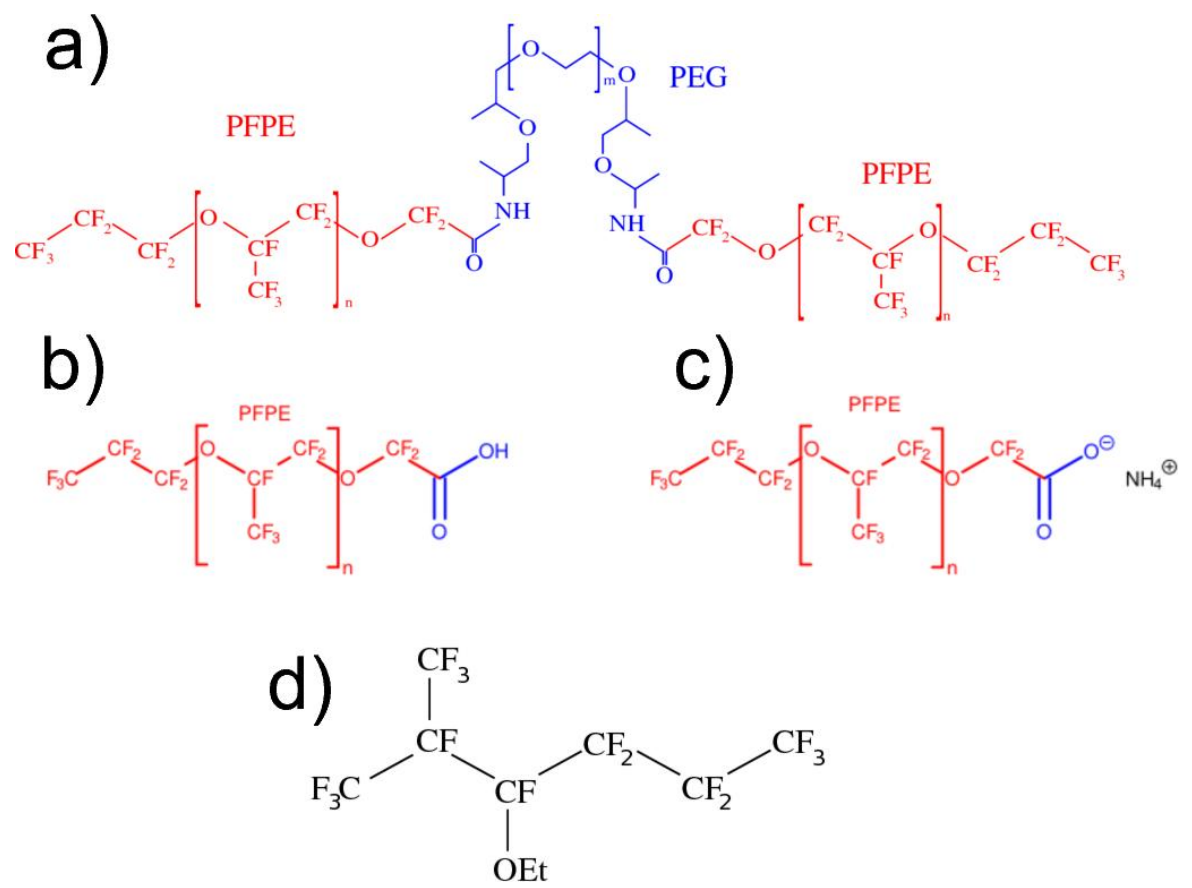

Figure 6.2 Chemical structure of the perfluorinated surfactants studied in this chapter. (a) PEG-PFPE with $n=12, m=34$ [73]. (b) Krytox-H with $n=12$ with its $\mathrm{H}^{+}$counterion. (c) Krytox$\mathrm{NH}_{4}$ with $n=12$ with its $\mathrm{NH}_{4}^{+}$counterion. (d) HFE-7500 fluorinated in which surfactant are dissolved to prepare the solutions.

terminated by a carboxylate $\mathrm{COO}^{-}$function. The molecules are neutralized by a counterion: $\mathrm{H}^{+}$in the case of Krytox-H and $\mathrm{NH}_{4}^{+}$in the case of Krytox- $\mathrm{NH}_{4}$. The total molar mass for these two molecules is roughly the same and is assumed to be of the order of the PFPE chain $M_{\mathrm{PPPE}}=2500 \mathrm{~g} \cdot \mathrm{mol}^{-1}$.

The molecule PEG-PFPE is synthesized from Krytox- $\mathrm{NH}_{4}$, two molecules are attached by a Polyethylene glycol structure (PEG) 34 units long with a molecular mass of $M_{\mathrm{PEG}-\mathrm{PFPE}}=$ $12500 \mathrm{~g} \cdot \mathrm{mol}^{-1}$.

\subsection{Method}

We used the pendant drop method to study the dynamics surface tension of the three perfluorosurfactant. The theoretical background for this method is described in chapter 2, All the chemicals are used as received without further purification. Surfactants are dissolved into a fluorinated oil, HFE-7500 the structure is shown in figure 6.2. The density ratio between HFE phase and the water phase is $\Delta \rho=\rho_{\mathrm{HFE}}-\rho_{\mathrm{H}_{2} \mathrm{O}}=0.62 \mathrm{~g} \cdot \mathrm{L}^{-1}$, and impels the production of 
a pendant drop of the HFE-oil into water. The overall description of the apparatus is show in chapter 5. A quartz cuvette is filled with aqueous phase. Before each measurement the cuvette is rinsed with ethanol, millipore water, and finally with the aqueous phase to be tested. The glass syringe and the tubing used to produce the droplet are rinsed with pure HFE and with the solution of surfactant in oil being tested. All experiments are performed under constant temperature $T=21-22{ }^{\circ} \mathrm{C}$, in order to extract the corresponding isotherms.

\subsection{Diffusion-limited adsorption and scalings}

To further analyze the measurements of the dynamic surface tension, we refer to the diffusionlimited adsorption model equations originally derived by Ward and Tordai [14, 29], and described in chapter 3 . The early time kinetics of the surface tension, in diffusive processes are given by:

$$
\gamma=\gamma_{0}-2 n R T C \sqrt{\frac{D t}{\pi}}
$$

with the prefactor $\zeta$ :

$$
\zeta=2 n R T C \sqrt{\frac{D}{\pi}}
$$

where $\Gamma_{\mathrm{eq}}$ is the equilibrium surface coverage and $D$ is the diffusion coefficient of the surfactant molecule.

We recall as well that the kinetics close to equilibrium or late kinetics is given by [14]:

$$
\gamma-\gamma_{e q}=\frac{n R T \Gamma_{e q}^{2}}{C} \sqrt{\frac{\pi}{4 D t}}=\lambda t^{-1 / 2}
$$

For all concentrations tested in this chapter, we have $0.8<\Gamma_{\text {eq }} / \Gamma_{\infty}<1$. Thus, the typical scale for the surface tension is given by $n R T \Gamma_{\infty}$ which is used to re-write the prefactor $\lambda$ as a characteristic time of adsorption for the late kinetics:

$$
\frac{\gamma-\gamma_{e q}}{n R T \Gamma_{\infty}}=\sqrt{\tau / t}
$$

with the characteristic time for the adsorption being:

$$
\tau=\frac{\pi \Gamma_{\infty}^{2}}{4 D C^{2}}
$$


In addition we use the Langmuir-von Szyszkowsky equation to describe the equilibrium of the adsorption process:

$$
\Pi=n R T \Gamma_{\infty} \ln (1+\kappa C)
$$

This set of equation will be used in the following analyses to describe the kinetics.

\subsubsection{Block copolymer PEG-PFPE}

In this section we make the characterization of the dynamic of adsorption of one neutral macromolecule PEG-PFPE surfactant. The interfacial tension $\gamma$ is measured as a function of time figure 6.3 , for various surfactant concentrations $C$ in the range $C=1.3 \times 10^{-3} \mathrm{~mol} \cdot \mathrm{m}^{-3}$ to $C=1.04 \mathrm{~mol} \cdot \mathrm{m}^{-3}$. The surface tension decays from $\gamma_{0}=49.5 \pm 0.5 \mathrm{mN} \cdot \mathrm{m}^{-1}$ to the equilibrium value $\gamma_{\mathrm{eq}}(C) . \gamma_{\mathrm{eq}}$ decreases with increasing surfactant concentration and the equilibrium time is decreased for higher surfactant concentrations. For lower concentrations, the equilibrium values are sometimes not reached after $10^{5} \mathrm{~s}$.

\section{Kinetics of adsorption}

We are first interested in the late kinetic properties of the diffusion of the PEG-PFPE macromolecule to the interface. The dynamic surface tension measurements are performed for bulk surfactant concentration ranging from $C_{\mathrm{PEG}-\mathrm{PFPE}}=1.3 \times 10^{-3} \mathrm{~mol} \cdot \mathrm{m}^{-3}$ to $C_{\mathrm{PEG}-\mathrm{PFPE}}=$ $1.04 \mathrm{~mol} \cdot \mathrm{m}^{-3}$. The corresponding dynamics are presented in figure 6.3 (a)

We used a fit according to the equation 6.3 to extract $\gamma_{\mathrm{eq}}$ and the prefactor $\lambda$ for the late surface tension kinetics. We found that these kinetics are in agreement with a $t^{-1 / 2}$, as shown in figure 6.3 (c).

The late kinetics characteristic times are presented in figure 6.3 (d). We find two asymptotic regimes where the relationship $\tau \sim C^{-2}$, consistent with a diffusion limited regime, is recovered. At high concentrations (above the $\mathrm{CMC}$ ), we obtain a diffusion coefficient $D_{\text {micelle }}=6 \times 10^{-12} \mathrm{~m}^{2} \cdot \mathrm{s}^{-1}$, compatible with the diffusion coefficients of micelles of sizes $100 \mathrm{~nm}$ that have been measured using Dynamic Light Scattering. At low concentrations $C<$ $0.15 \mathrm{~mol} \cdot \mathrm{m}^{-3}$ (below the CMC), the diffusion coefficient is $D_{\text {monomer }}=1.5 \times 10^{-9} \mathrm{~m}^{2} \cdot \mathrm{s}^{-1}$. This value exceeds by about one order of magnitude a typical value that can be expected for such a nanometer sized molecule. Between the two asymptotic behaviors we observe a crossover region where the kinetics is not significantly affected by the surfactant concentration. These results indicate that micelles contribute to the decrease of surface tension, provided 


\begin{tabular}{ccc}
\hline Parameter & Value & Units \\
\hline \hline$M_{w}$ & 12500 & $\mathrm{~g} \cdot \mathrm{mol}^{-1}$ \\
$\Gamma_{\infty}$ & $3.4 \times 10^{-6}$ & $\mathrm{~mol} \cdot \mathrm{m}^{-2}$ \\
$\kappa$ & $3 \times 10^{3}$ & $\mathrm{~m}^{3} \cdot \mathrm{mol}^{-1}$ \\
$\mathrm{CMC}$ & $1.5 \times 10^{-3}$ & $\mathrm{~mol} \cdot \mathrm{m}^{-3}$ \\
$D_{C \ll C M C}^{*}$ & $1.5 \times 10^{-9}$ & $\mathrm{~m}^{2} \cdot \mathrm{s}^{-1}$ \\
$D_{C \gg C M C}$ & $6 \times 10^{-12}$ & $\mathrm{~m}^{2} \cdot \mathrm{s}^{-1}$ \\
\hline
\end{tabular}

Table 6.1 PEG-PFPE surfactant parameters obtained from pendant drop tensiometry. ${ }^{*}$ This value is an apparent diffusion coefficient, possibly accounting for convective currents present in the initial stage of the droplet creation and that shift the results.

that they have sufficient time to diffuse to the interface from the bulk and the dissociation of micelles is not the limiting step as observed for other non-ionic micelles [34, 74].

In the second step we are interested in the early kinetics of the dynamics surface tension. We fit here the experimental dynamics for concentration $C_{\mathrm{PEG}-\mathrm{PFPE}}=1.3 \times 10^{-3} \mathrm{~mol} \cdot \mathrm{m}^{-3}$ to $C_{\mathrm{PEG}-\mathrm{PFPE}}=2.6 \times 10^{-2} \mathrm{~mol} \cdot \mathrm{m}^{-3}$ by a $\zeta t^{1 / 2}$ power law, and plot $\zeta$ as a function of the concentration, thereby extracting the diffusion coefficient. See figure 6.2 (a) and (b). We find a linear relation between $\zeta$ and $C$, also consistent with the a diffusion-limited regime as presented in the previous section. However the value of the diffusion coefficient $D_{\text {monomer }}=$ $1 \times 10^{-8} \mathrm{~m}^{2} \cdot \mathrm{s}^{-1}$ is non physical. The error is probably the result of the convective currents induced at the initial time of the drop formation. A similar observation has been made for other surfactants in pendant drop experiments [75]. The apparent "superdiffusivity" of our molecule pointed out here was also visible in the late kinetics.

\section{Equilibrium}

Finally, from the equilibrium surface tension values $\gamma_{\mathrm{eq}}$, extracted with equation 6.3 , we obtain the Langmuir isotherm for this surfactant. We determine the $C_{\mathrm{CMC}}=1.5 \times 10^{-3} \mathrm{~mol} \cdot \mathrm{m}^{-3} \pm$ $0.5 \times 10^{-3} \mathrm{~mol} \cdot \mathrm{m}^{-3}$ for this surfactant from on-set of the plateau region in the Langmuir isotherm. We then apply the Langmuir-von Szyszkowsky equation (equation 6.6) to the region below the $\mathrm{CMC}$ in order to obtain the parameters of the equilibrium. We find a good fit of our experimental data for the values of the interfacial maximum coverage $\Gamma_{\infty}=$ $3.4 \times 10^{-6} \mathrm{~mol} \cdot \mathrm{m}^{-2}$ and for the interfacial affinity $\kappa=3 \times 10^{3} \mathrm{~m}^{3} \cdot \mathrm{mol}^{-1}$. See figure 6.3 (b). The parameters which describe the surfactant interfacial properties are summarized in table 
6.1 .

In summary, the pendant drop experiments show that the interface equilibrium is described in a good approximation by a standard Langmuir isotherm while the dynamics of adsorption display scalings that are compatible with the diffusion limited kinetics. However, the large values of the diffusion parameters obtained with this model, particularly in the early kinetics, suggest the presence of additional contributions to the diffusion at the initial stage of the adsorption. When the interface is created, the presence of convective currents linked to the flow displacements near the interface speed up momentarily the adsorption of surfactant. In addition our description do not describe the effect of the interface curvature which has been shown to modify the processes of adsorption. As a result the scalings are in good agreement with the scaling a of a diffusive process. We continue this study by applying this model for the two simple PFPE chains Krytox-H and Krytox- $\mathrm{NH}_{4}$. 

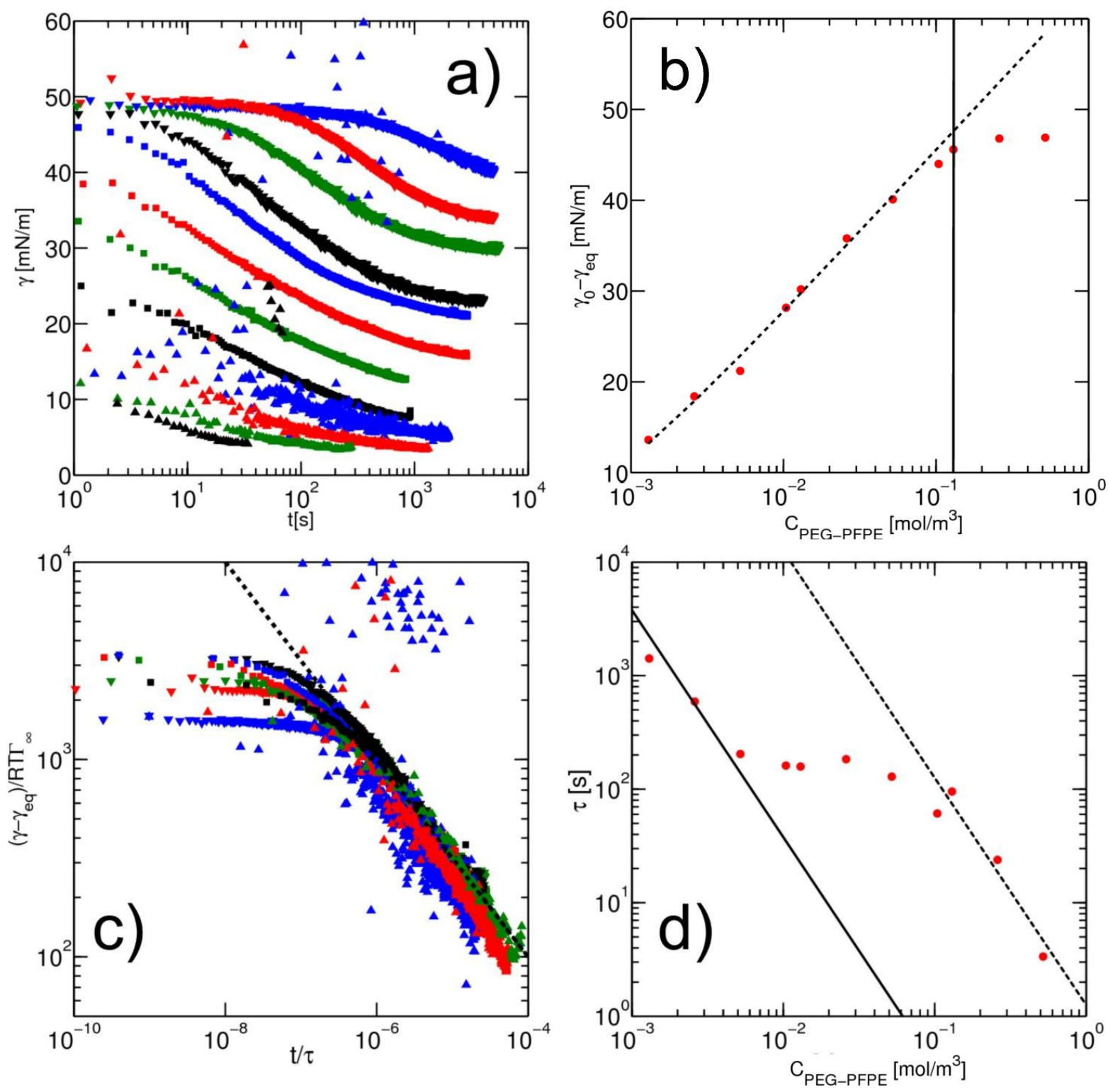

Figure 6.3 (a) Dynamics tensiometry in pendant drop method for the following concentrations: $C_{\text {PEG-PFPE }}=1.3 \times 10^{-3} \mathrm{~mol} \cdot \mathrm{m}^{-3}(\boldsymbol{\nabla}) ; C_{\text {PEG-PFPE }}=2.6 \times 10^{-3} \mathrm{~mol} \cdot \mathrm{m}^{-3}, \quad(\boldsymbol{\nabla}) ;$ $C_{\mathrm{PEG}-\mathrm{PFPE}}=5.2 \times 10^{-3} \mathrm{~mol} \cdot \mathrm{m}^{-3},(\boldsymbol{\nabla}) ; C_{\mathrm{PEG}-\mathrm{PFPE}}=10.4 \times 10^{-3} \mathrm{~mol} \cdot \mathrm{m}^{-3},(\mathbf{v}) ;$ $C_{\text {PEG-PFPE }}=1.3 \times 10^{-2} \mathrm{~mol} \cdot \mathrm{m}^{-3}, \quad(\mathbf{\square}) ; C_{\text {PEG-PFPE }}=2.6 \times 10^{-2} \mathrm{~mol} \cdot \mathrm{m}^{-3}, \quad(\square) ;$ $C_{\text {PEG-PFPE }}=5.2 \times 10^{-2} \mathrm{~mol} \cdot \mathrm{m}^{-3},(\square) ; C_{\mathrm{PEG}-\mathrm{PPPE}}=0.104 \mathrm{~mol} \cdot \mathrm{m}^{-3},(\square) ; C_{\mathrm{PEG}-\mathrm{PFPE}}=$ $0.13 \mathrm{~mol} \cdot \mathrm{m}^{-3},(\Delta) ; C=0.26 \mathrm{~mol} \cdot \mathrm{m}^{-3},(\Delta) ; C_{\mathrm{PEG}-\mathrm{PFPE}}=0.52 \mathrm{~mol} \cdot \mathrm{m}^{-3},(\Delta)$; $C_{\text {PEG-PFPE }}=1.04 \mathrm{~mol} \cdot \mathrm{m}^{-3},(\boldsymbol{\Lambda})$. (b) Equilibrium surface tension extracted from the long term kinetics equation 6.3. The doted line is a fitting of the linear part of the isotherm, with the Langmuir equation 6.6 with coefficients $\Gamma_{\mathrm{eq}}=3.4 \mathrm{~mol} \cdot \mathrm{m}^{-2}$ and $\kappa=3.1 \times 10^{3} \mathrm{~mol}^{3} \cdot \mathrm{m}^{-2}$. The black line represent the CMC falling around $0.15 \mathrm{~mol} \cdot \mathrm{m}^{-3}$. (c) Dynamic surface tension against the rescaled time $t / \tau$. The long term dynamics are collapsing on a $t^{-0.5}$ power law (dashed line), equation 6.3. (d) Evolution of the characteristic time $\tau$ with the surfactant concentration. Line represent theoretical values of $\tau$, equation 6.4, for different diffusion coefficients. The monomer diffusion is represented by the solid line with $D_{\text {monomer }}=1.5 \times 10^{-9} \mathrm{~m}^{2} \cdot \mathrm{s}^{-1}$. The doted line correspond to a process of micellar diffusion with $D_{\text {micelles }}=6 \times 10^{-12} \mathrm{~m}^{2} \cdot \mathrm{s}^{-1}$. 

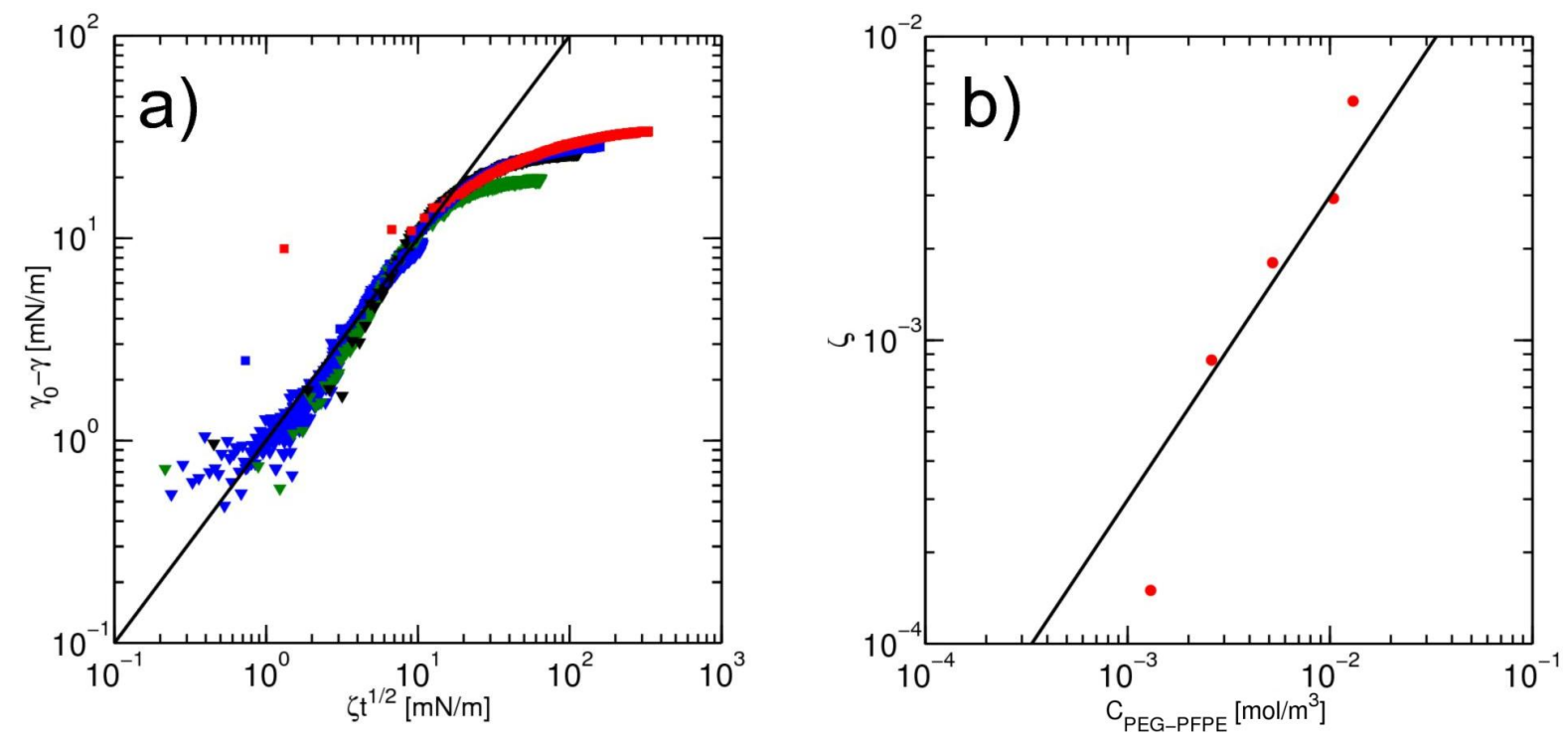

Figure 6.4 (a) Dynamic surface tension rescaled by the prefactor $\zeta$ for the following surfactant concentrations: $C_{\text {PEG-PFPE }}=1.3 \times 10^{-3} \mathrm{~mol} \cdot \mathrm{m}^{-3}(\mathbf{\nabla}) ; C_{\mathrm{PEG}-\mathrm{PFPE}}=5.2 \times 10^{-3} \mathrm{~mol} \cdot \mathrm{m}^{-3}$, $(\boldsymbol{\nabla}) ; C_{\mathrm{PEG}-\mathrm{PFPE}}=10.4 \times 10^{-3} \mathrm{~mol} \cdot \mathrm{m}^{-3},(\mathbf{v}) ; C_{\mathrm{PEG}-\mathrm{PFPE}}=1.3 \times 10^{-2} \mathrm{~mol} \cdot \mathrm{m}^{-3},(\mathbf{\square})$; $C_{\mathrm{PEG}-\mathrm{PFPE}}=2.6 \times 10^{-2} \mathrm{~mol} \cdot \mathrm{m}^{-3},(\mathbf{\square})$. All kinetics collapse on a slope 1 power predicted from equation 6.2. (b) Values of the prefactor $\zeta$ in function of the surfactant concentration. The solid black line is a fit of equation 6.2 with parameter $D_{\text {monomer }}=1 \times 10^{-8} \mathrm{~m}^{2} \cdot \mathrm{s}^{-1}$ 


\subsection{Charged surfactant Krytox-H and Krytox- $-\mathrm{NH}_{4}$}

In the previous section we have seen how the main thermodynamical parameters could be extracted for one macromolecule. In this section we carry the same analysis for the two molecules that serve as basis for the synthesis of the PEG-PFPE molecules. With this study we would like to unveil the possible effects of the charge dissociation at the interface.

The Krytox-H and Krytox- $\mathrm{NH}_{4}$ molecules are built on the same model: a PFPE chain is linked to a carboxylate group $\left(\mathrm{COO}^{-}\right)$. The overall neutrality is ensured by the presence of a counter ion, $\left(\mathrm{H}^{+}\right)$for Krytox-H molecule and an ammonium ion $\left(\mathrm{NH}_{4}^{+}\right)$for Krytox- $\mathrm{NH}_{4}$. These molecules are overall neutral in the oil phase. Therefore we expect that their adsorption kinetics should be similar to PEG-PFPE molecule, with different diffusion coefficients given the difference in the molecule size. However, we can already predict that a charge dissociation will occur upon the adsorption at the water interface. We are interested to study the role that may be played by this additional phenomenon, and compare the influence that the different ions $\mathrm{H}^{+}$and $\mathrm{NH}_{4}^{+}$can have on the adsorption.

\subsubsection{General form of the dynamics}

In order to better understand these dissociation mechanisms and the consequences on the surface tension dynamics, we investigate the adsorption at the oil / millipore water interface, and at oil / brine interface.

Krytox-H: The figure 6.5 resumes the dynamics extracted for surfactant concentration between $C_{\mathrm{K}-\mathrm{H}}=6.5 \times 10^{-4} \mathrm{~mol} \cdot \mathrm{m}^{-3}$ to $C_{\mathrm{K}-\mathrm{H}}=3.2 \mathrm{~mol} \cdot \mathrm{m}^{-3}$, in pure water (figure $6.5(\mathrm{~b})$ ), and in presence of $C_{\mathrm{NaCl}}=10^{2} \mathrm{~mol} \cdot \mathrm{m}^{-3}$ of $\mathrm{NaCl}$ salt dissolved in the water phase, see figure 6.5 (a). Depending on the surfactant concentration the equilibrium is reached for both experimental cases within a time range $t=10^{3}-10^{4} \mathrm{~s}$. In the presence of additional charges in the water phase, the overall values of the equilibrium surface tension are decreased. Therefore the higher range of concentration, above $C_{\mathrm{K}-\mathrm{H}}=1.3 \times 10^{-1} \mathrm{~mol} \cdot \mathrm{m}^{-3}$, results in equilibrium surface tensions which are too low to be reliably measurable with pendant drop method.

Krytox- $\mathrm{NH}_{4}$ : The surface tension dynamics, for millipore water, and in presence of salt linked to Krytox- $\mathrm{NH}_{4}$ experiments, are presented on figure 6.6 (a) and (b). The same general comments about the decreasing values of the equilibrium surface tension and the range of concentration accessible in the experiment done with in presence of salt can be made. In addition, a noticeable difference on the dynamics of surface tension can be made. First, the dynamics of 
$\mathrm{H}_{2} \mathrm{O}+\mathrm{NaCl} 0.1 \mathrm{~mol} / \mathrm{L}$

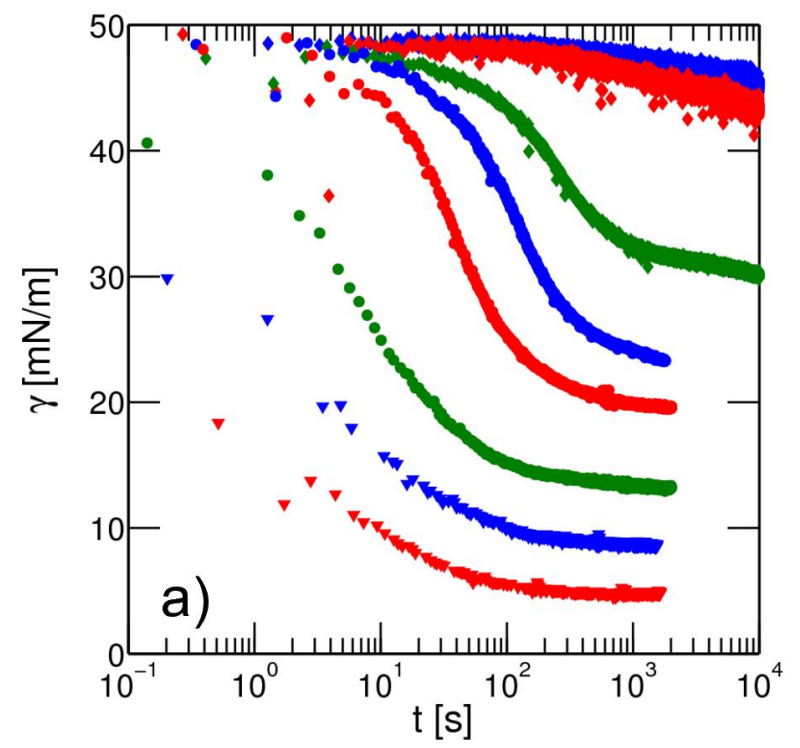

$\mathrm{H}_{2} \mathrm{O}$

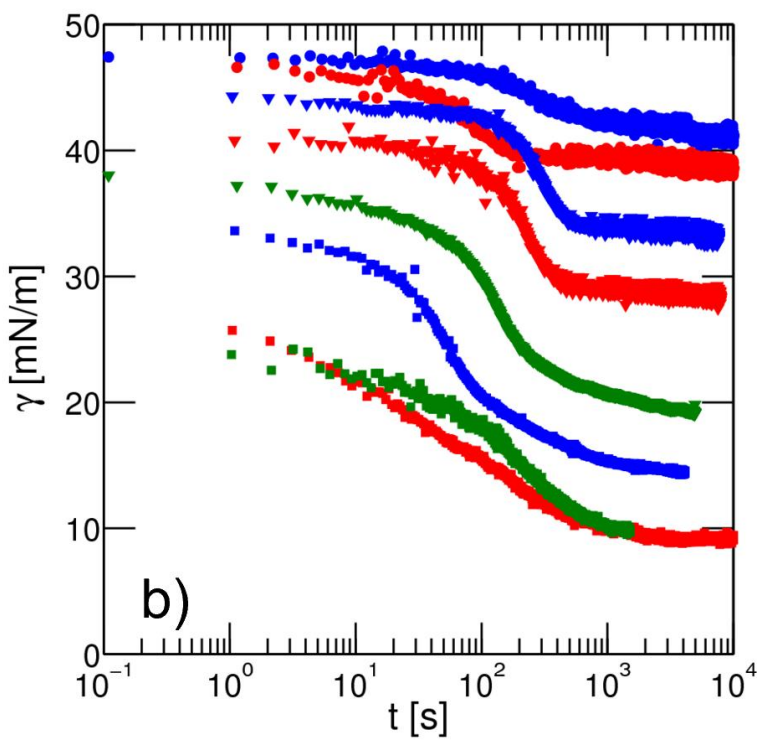

Figure 6.5 Dynamic surface tension extracted from pendant drop method for concentrations: $\quad C_{\mathrm{K}-\mathrm{H}}=6.5 \times 10^{-4} \mathrm{~mol} \cdot \mathrm{m}^{-3}(\diamond) ; \quad C_{\mathrm{K}-\mathrm{H}}=1.3 \times 10^{-3} \mathrm{~mol} \cdot \mathrm{m}^{-3}(\diamond) ;$ $C_{\mathrm{K}-\mathrm{H}}=3.2 \times 10^{-3} \mathrm{~mol} \cdot \mathrm{m}^{-3}(\diamond) ; \quad C_{\mathrm{K}-\mathrm{H}} \quad=\quad 6.5 \times 10^{-3} \mathrm{~mol} \cdot \mathrm{m}^{-3}(\bullet) ;$ $C_{\mathrm{K}-\mathrm{H}}=1.3 \times 10^{-2} \mathrm{~mol} \cdot \mathrm{m}^{-3}(\bullet) ; \quad C_{\mathrm{K}-\mathrm{H}}=3.2 \times 10^{-2} \mathrm{~mol} \cdot \mathrm{m}^{-3}(\bullet) ;$ $C_{\mathrm{K}-\mathrm{H}}=6.5 \times 10^{-2} \mathrm{~mol} \cdot \mathrm{m}^{-3}(\mathbf{v}) ; \quad C_{\mathrm{K}-\mathrm{H}}=1.3 \times 10^{-1} \mathrm{~mol} \cdot \mathrm{m}^{-3}(\mathbf{\nabla}) ;$ $C_{\mathrm{K}-\mathrm{H}}=3.2 \times 10^{-1} \mathrm{~mol} \cdot \mathrm{m}^{-3}(\mathbf{\nabla}) ; \quad C_{\mathrm{K}-\mathrm{H}}=6.5 \times 10^{-1} \mathrm{~mol} \cdot \mathrm{m}^{-3}(\boldsymbol{\nabla}) ; \quad C_{\mathrm{K}-\mathrm{H}}=$ $1.3 \mathrm{~mol} \cdot \mathrm{m}^{-3}(\square) ; C_{\mathrm{K}-\mathrm{H}}=3.2 \mathrm{~mol} \cdot \mathrm{m}^{-3}(\square)$. The measurement are processed with: (a) a solution of $C_{\mathrm{NaCl}}=10^{2} \mathrm{~mol} \cdot \mathrm{m}^{-3}$ as aqueous phase and (b) millipore water.

the surface tension present a reproducible non monotonic feature. A reproducible "bounce" is visible around $t \sim 100 \mathrm{~s}$, in the range of concentration $C_{\mathrm{K}}=1.3 \times 10^{-1}-5.2 \times 10^{-1} \mathrm{~mol} \cdot \mathrm{m}^{-3}$. This bounce seems to disappear when additional charges are added in the water phase.

From this graph we can qualitatively draw two main conclusions:

- First, the difference in equilibrium surface tensions $\gamma_{\mathrm{eq}}$ for the millipore water phase and in presence of salt indicates that the equilibrium state of the interface depends on the ion concentration in water phase.

- Second, the presence of the "bounce" in the case of Krytox- $\mathrm{NH}_{4}$ is the sign that an additional effect other than the molecular diffusion is influencing the transport of matter to the interface.

In the following part we will quantitatively analyze the equilibrium state and the kinetics of the adsorption for both molecules in order to draw conclusions about the overall adsorption 

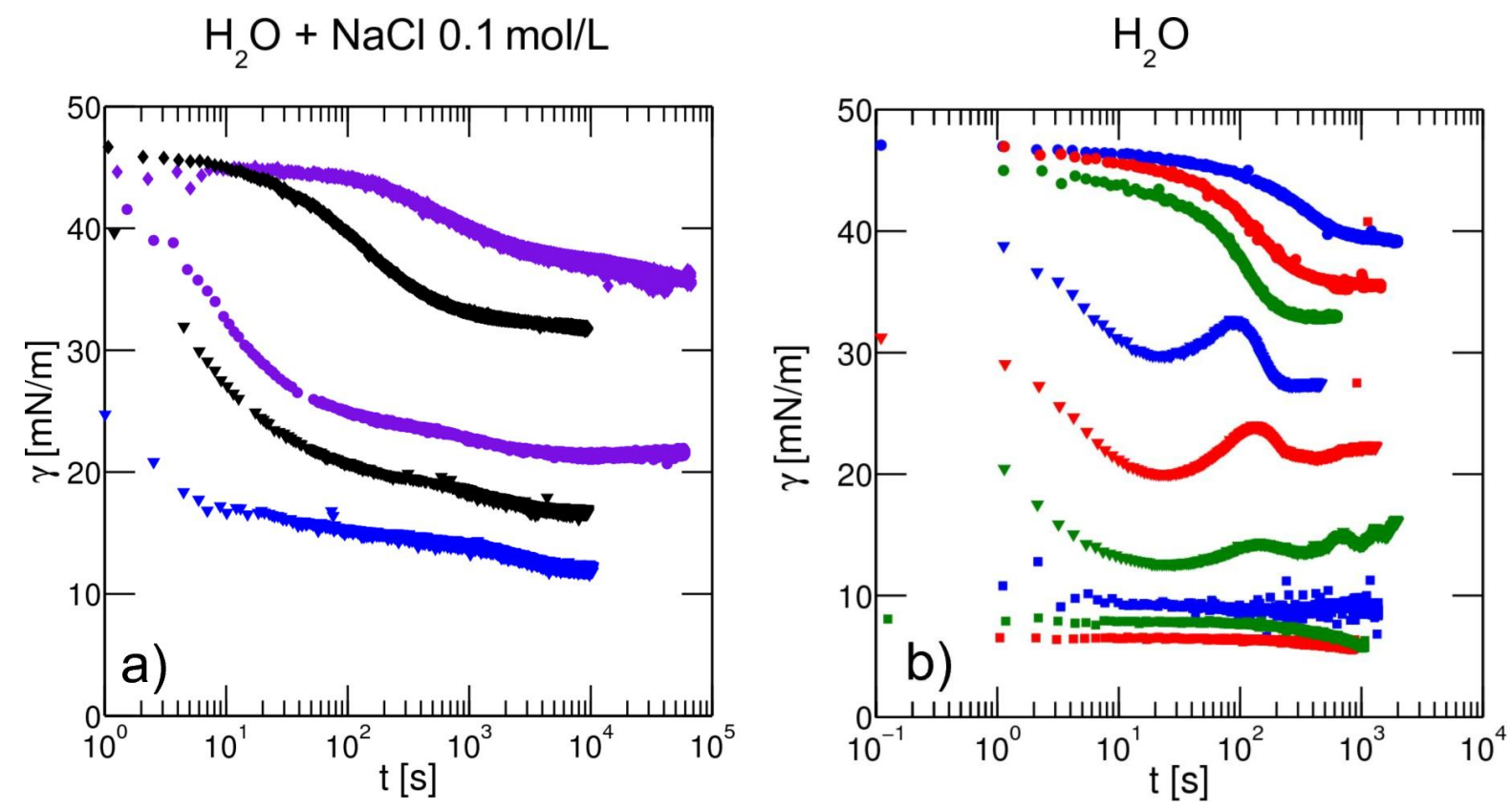

Figure 6.6 Dynamic surface tension for concentrations: $C_{\mathrm{K}-\mathrm{NH}_{4}}=3.2 \times 10^{-3} \mathrm{~mol} \cdot \mathrm{m}^{-3}(\diamond)$; $C_{\mathrm{K}-\mathrm{NH}_{4}}=6.5 \times 10^{-3} \mathrm{~mol} \cdot \mathrm{m}^{-3}(\bullet) ; \quad C_{\mathrm{K}-\mathrm{NH}_{4}}=1.3 \times 10^{-2} \mathrm{~mol} \cdot \mathrm{m}^{-3}(\bullet) ;$ $C_{\mathrm{K}-\mathrm{NH}_{4}}=2.6 \times 10^{-2} \mathrm{~mol} \cdot \mathrm{m}^{-3}(\bullet) ; \quad C_{\mathrm{K}-\mathrm{NH}_{4}}=3.2 \times 10^{-2} \mathrm{~mol} \cdot \mathrm{m}^{-3}(\bullet) ;$ $C_{\mathrm{K}-\mathrm{NH}_{4}}=5.2 \times 10^{-2} \mathrm{~mol} \cdot \mathrm{m}^{-3}(\bullet) ; \quad C_{\mathrm{K}-\mathrm{NH}_{4}}=6.5 \times 10^{-2} \mathrm{~mol} \cdot \mathrm{m}^{-3}(\mathbf{\nabla}) ;$ $C_{\mathrm{K}-\mathrm{NH}_{4}}=1.3 \times 10^{-1} \mathrm{~mol} \cdot \mathrm{m}^{-3}(\mathbf{v}) ; C_{\mathrm{K}-\mathrm{NH}_{4}}=2.6 \times 10^{-1} \mathrm{~mol} \cdot \mathrm{m}^{-3}(\mathbf{\nabla}) ; C_{\mathrm{K}-\mathrm{NH}_{4}}=$ $5.2 \times 10^{-1} \mathrm{~mol} \cdot \mathrm{m}^{-3}(\mathbf{v}) ; C_{\mathrm{K}-\mathrm{NH}_{4}}=1.3 \mathrm{~mol} \cdot \mathrm{m}^{-3}(\boldsymbol{\square}) ; C_{\mathrm{K}-\mathrm{NH}_{4}}=2.6 \mathrm{~mol} \cdot \mathrm{m}^{-3}(\mathbf{\square}) ;$ $C_{\mathrm{K}-\mathrm{NH}_{4}}=5.2 \mathrm{~mol} \cdot \mathrm{m}^{-3}(\square)$. The measurement are processed with: (a) a solution of $C_{\mathrm{NaCl}}=10^{2} \mathrm{~mol} \cdot \mathrm{m}^{-3}$ as aqueous phase and (b) millipore water.

mechanisms for both molecules.

\subsubsection{Equilibrium}

In this part, we focus on the properties of the dynamic equilibrium of the surfactant adsorption / desorption upon the oil / water interface. We apply the same methods as described for the PEG-PFPE molecules in order to extract the equilibrium surface tensions $\gamma_{\mathrm{eq}}$. The figure 6.7 shows the four Langmuir isotherms corresponding to the four different experimental conditions (two surfactants and two different water phases). We choose to fit these isotherms with the Langmuir equation (equation 6.6) in order to obtain the parameters of the equilibrium state equation $\kappa$ and $\Gamma_{\infty}$. Table 6.2 gathers the corresponding fitting parameters. 
$\mathrm{H}_{2} \mathrm{O}+\mathrm{NaCl} 0.1 \mathrm{~mol} / \mathrm{L}$
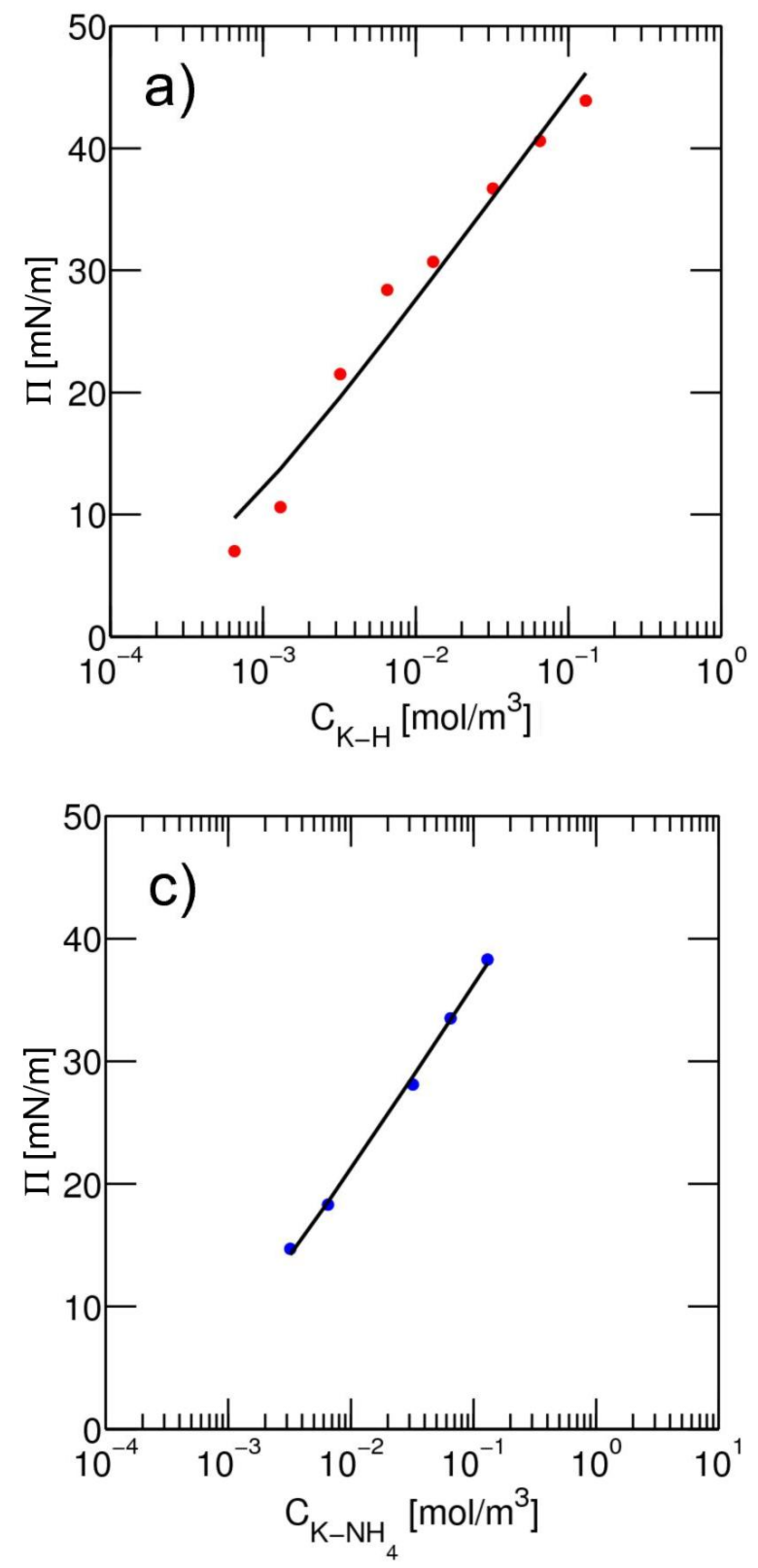

$\mathrm{H}_{2} \mathrm{O}$
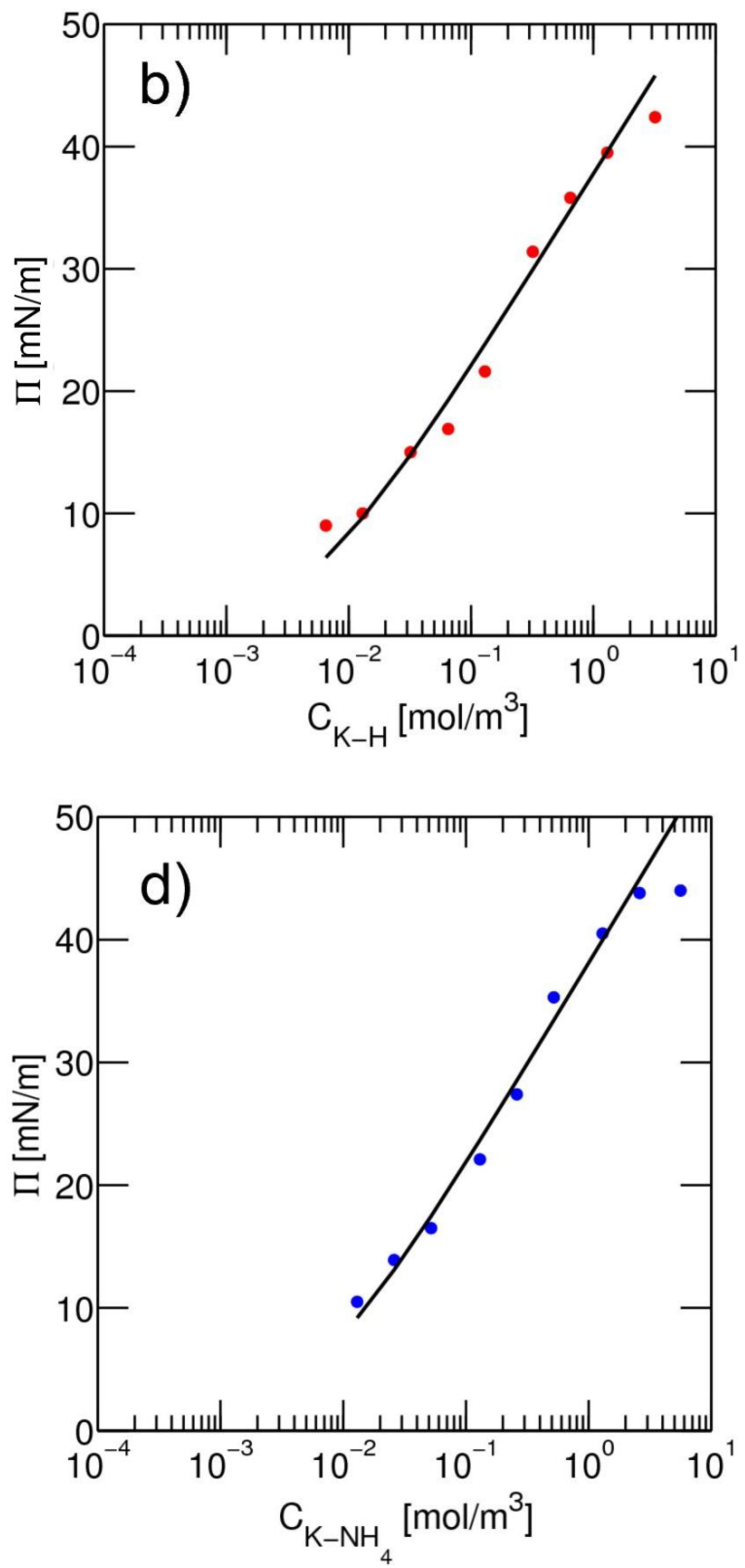

Figure 6.7 Equilibrium interfacial pressure in function of the surfactant concentrations for Krytox-H $(\bullet)$, and Krytox- $\mathrm{NH}_{4}(\bullet)$ both for two different aqueous salt concentration. A fitting of the Langmuir-von Szyszkowsky equation (equation 6.6) allows us to obtain the theoretical interface maximum coverage $\Gamma_{\infty}$, and the adsorption reaction constant, or the interface affinity K 


\begin{tabular}{cccccc}
\hline Molecule & & $\mathrm{n}$ & $\kappa\left[\mathrm{m}^{3} \cdot \mathrm{mol}^{-1}\right]$ & $\Gamma_{\infty}\left[\mathrm{mol} \cdot \mathrm{m}^{-2}\right]$ & $\mathrm{C}_{\mathrm{CMC}}\left[\mathrm{mol} \cdot \mathrm{m}^{-3}\right]$ \\
\hline \hline \multirow{2}{*}{ Krytox-H } & $\mathrm{H}_{2} \mathrm{O}$ & 2 & 230 & $1.4 \times 10^{-6}$ & \multirow{2}{*}{2} \\
\cline { 2 - 5 } & $\mathrm{NaCl}$ & 1 & 4290 & $3 \times 10^{-6}$ & \\
\hline \multirow{2}{*}{ Krytox-NH$_{4}$} & $\mathrm{H}_{2} \mathrm{O}$ & 2 & 190 & $1.4 \times 10^{-6}$ & \multirow{2}{*}{2} \\
\cline { 2 - 5 } & $\mathrm{NaCl}$ & 1 & 2370 & $2.6 \times 10^{-6}$ & 2 \\
\hline
\end{tabular}

Table 6.2 Parameters of the surface tension equilibrium for both perfluorosurfactants Krytox-H and Krytox- $\mathrm{NH}_{4}$, obtained by fitting of the Langmuir-von Szyszkowsky (equation 6.6. The values of $n$ are chosen according to the conditions described in chapter 2] and to reference[14].

Krytox-H: In both cases the description of the equilibrium is fitted by the Langmuir isotherm 6.6. We recover from the case with millipore water the parameters $\Gamma_{\infty}=1.4 \times 10^{-6} \mathrm{~mol} \cdot \mathrm{m}^{-2}$ and $\kappa=230 \mathrm{~m}^{3} \cdot \mathrm{mol}^{-1}$

Then we extract the parameters $\Gamma_{\infty}=3 \times 10^{-6} \mathrm{~mol} \cdot \mathrm{m}^{-2} \kappa=4290 \mathrm{~m}^{3} \cdot \mathrm{mol}^{-1}$ for the case with added salt.

Krytox- $\mathrm{NH}_{4}$ : The same analysis is carried for the equilibrium of molecule Krytox- $\mathrm{NH}_{4}$. Comparable values are found for the maximum surfactant coverage with $\Gamma_{\infty}=3 \times 10^{-6} \mathrm{~mol} \cdot \mathrm{m}^{-2}$ in presence of millipore water and $\Gamma_{\infty}=2.6 \times 10^{-6} \mathrm{~mol} \cdot \mathrm{m}^{-2}$ in presence of salt in the aqueous phase. Once more the presence of charges is found to affect the affinity of the molecule to the interface. We found the values $\kappa=190 \mathrm{~m}^{3} \cdot \mathrm{mol}^{-1}$ for the case without salt and $\kappa=2370 \mathrm{~m}^{3} \cdot \mathrm{mol}^{-1}$ in presence of salt. Here as well the values are consistent with the one found for Krytox-H.

The values of the equilibrium present a deviation from the Langmuir model for concentrations above $C_{\mathrm{CMC}}=2 \mathrm{~mol} \cdot \mathrm{m}^{-3}$, probably due to the presence of micellar aggregates for concentrations above the CMC.

For the same experimental conditions, the difference of counter ions does not contribute to a change in the equilibrium. The process of charge dissociation is extremely short compared to the time scale of the adsorption (electronic processes occur usually in time scale on the order of $10^{-9} \mathrm{~s}$ ). Therefore no effects on the kinetic of surfactant adsorption, or the equilibrium, can be attributed to the process of charges dissociation. Once the charges dissociation has occurred, an identical charged PFPE chain for both surfactants remains at the interface. Here only effect coming from the presence of additional electrolytes in the water phase can be seen. 


\section{Screening of electrostatic interactions between surfactant molecules at the interface}

The electrostatic forces between two surfactant molecules are large enough to ensure an electrostatic repulsion between neighboring molecules. At the scale of the interface, the presence of charges in the water phase and the resulting screening of the electrostatic forces can change dramatically the global repartition of the surfactant at the interface. The distance between two neighboring molecules $d_{\mathrm{m}}$ at the interface can be estimated by: $d_{\mathrm{m}}=\mathcal{N}_{\mathrm{a}} / \Gamma_{\infty}$ We find the values $d_{\mathrm{m}}=1.2 \mathrm{~nm}$ for the case without salt, and $d_{\mathrm{m}}=0.5 \mathrm{~nm}$ for the case with salt. This result can be understood considering the electrostatic properties of the PFPE chains when adsorbed at the interface. With increasing electrolyte concentration the electrostatic interactions between two neighboring molecules is progressively screened. In the approximation of point like particles, one can estimate the Debye length which characterizes the distance over which one charge feels the electric field produced by a neighboring molecules. The Debye length $\lambda_{\mathrm{D}}$ is defined as:

$$
\lambda_{\mathrm{D}}=\left[\frac{\epsilon_{0} \epsilon_{\mathrm{r}} k_{\mathrm{b}} T}{C_{\mathrm{x}}-\mathcal{N}_{\mathrm{a}} q^{2}}\right]^{1 / 2}
$$

with $\epsilon_{0}$ the vacuum permittivity, $\epsilon_{\mathrm{r}}$ the water relative permittivity, $C_{\mathrm{x}^{-}}$the concentration of negative electrolytes, $\mathcal{N}_{\mathrm{a}}$ the Avogadro number and $q$ the elementary charge.

In our range of electrolyte concentrations the Debye length is ranging from $\sim 100 \mathrm{~nm}$ in presence of millipore water with a $\mathrm{pH} \sim 5-6$, and $\lambda_{\mathrm{D}}=1 \mathrm{~nm}$ for a $\mathrm{NaCl}$ concentration $C_{\text {ions }}=10^{2} \mathrm{~mol} \cdot \mathrm{m}^{-3}$. These values confirm that the effective screening occurs between neighbor molecules at the interface in presence of salt. Therefore in the case where no additional electrolytes are present in the continuous phase the surfactant molecules organize themselves according to the electrostatic interactions. The value of $\Gamma_{\infty}$ is then smaller than in the presence of electrolytes. The sharp rise around the concentration $C_{\text {ion }}=10 \mathrm{~mol} \cdot \mathrm{m}^{-3}$ in figure 6.8 corresponds to the region for which the Debye length (here $\lambda_{\mathrm{D}} \sim 3 \mathrm{~nm}$ ) is approaching the intermolecular distance.

Interestingly, in this case the density of molecule per unit of surface, is still close to the one of the macromolecule PEG-PFPE. Therefore the presence of some residual interactions, possibly of steric nature, are still existing between neighboring molecules.

We study in more detail the effect of salt concentration on the surface affinity of the PFPE chain. We show on figure 6.8 the dynamic surface tension for a fixed surfactant concentration $C_{\mathrm{K}-\mathrm{H}}=1.3 \times 10^{-1} \mathrm{~mol} \cdot \mathrm{m}^{-3}$, for different salt concentrations in the water phase. We see that the equilibrium surface tension is being lowered by the presence of electrolytes in the water 
phase.
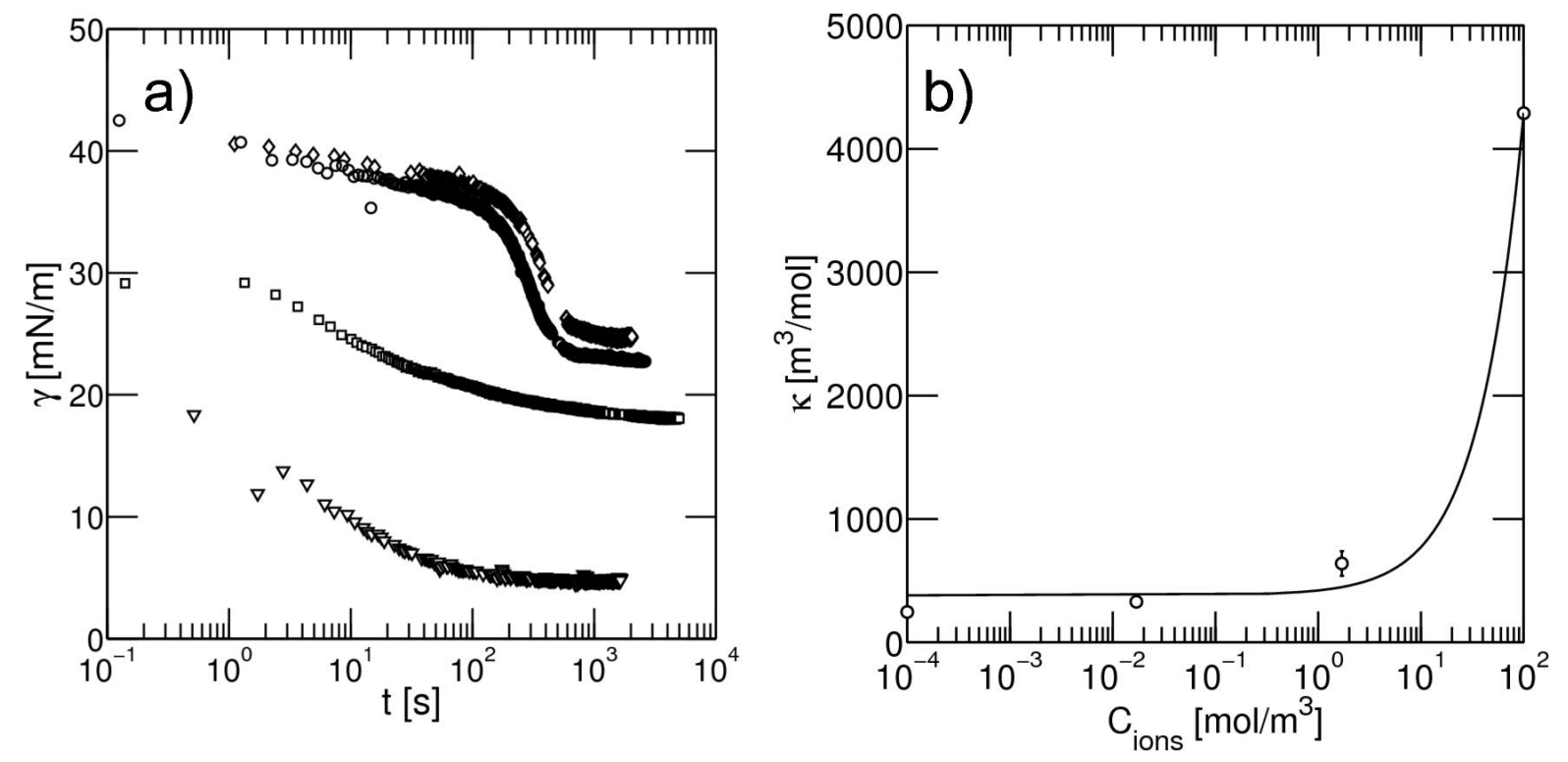

Figure 6.8 (a) Dependence of the equilibrium surface tension of molecule Krytox-H $\left(C_{\mathrm{K}-\mathrm{H}}=\right.$ $1.3 \times 10^{-1} \mathrm{~mol} \cdot \mathrm{m}^{-3}$ ) with ions concentration: $C_{\text {ion }}=10^{-4} \mathrm{~mol} \cdot \mathrm{m}^{-3}(\diamond) ; C_{\text {ion }}=$ $1.7 \times 10^{-2} \mathrm{~mol} \cdot \mathrm{m}^{-3}\left(\right.$ ( ) $; C_{\text {ion }}=1.7 \mathrm{~mol} \cdot \mathrm{m}^{-3}(\square) ; C_{\text {ion }}=100 \mathrm{~mol} \cdot \mathrm{m}^{-3}(\nabla)$. (b) Values of the surface affinity extracted from the Langmuir-von Szsyszkowsky equation (equation 6.6), using the values of $\kappa$. The difference of result found for the two values of $\kappa$ in comprised in the errorbar. The solid line is a guide for the eyes for the changes of $\kappa$ with $\mathrm{C}_{\text {ion }}$

We have defined in the previous chapter $\kappa=k_{\mathrm{ads}} / k_{\mathrm{des}}$, as the ratio of surfactant adsorption events to desorption events occurring at the interface. We know that a PFPE chain can not desorb from the interface to the oil phase without re-associating with a positive charge from the water phase. In this case two regions should be visible. One region is where the small concentration of electrolytes in the water phase makes the event of re-association dependent on the electrolyte concentration. Consequently, the values of $k_{\text {des }}$ should decrease and the value of $\kappa$ should increase with increasing electrolyte concentration. A second region should exist, above a certain threshold concentration, where the concentration of salt is large enough to ensure a high probability of re-association between the electrolytes and the surfactant at the interface at any concentration. In this case the behavior should tend to the one of a neutral molecule where the desorption is not dependent on the electrolyte concentration. Here a plateau of $\kappa$ should be visible.

We use the Langmuir-von Szyszkowszky equation, with fixed parameter $C_{\mathrm{K}-\mathrm{H}}$ to extract the variations of the interfacial affinity of the molecule as a function of the ion concentration. 
The two different values of $\Gamma_{\infty}$ are used for the determination of $\kappa$. The divergence between the two results is indicated in the errorbar. See figure 6.8 (b).

In our range of concentrations a jump for the value of $\kappa$ is visible between the concentrations $\mathrm{C}_{\mathrm{ion}}=1-100 \mathrm{~mol} \cdot \mathrm{m}^{-3}$ This region is the one for which the value of the Debye length $\lambda_{\mathrm{D}} \sim 3 \mathrm{~nm}$ is approaching the value of the intermolecular distance given by $\Gamma_{\infty}$. Therefore this transition corresponds tho the re-organization of the interface under the screening of the electrostatic forces between neighboring molecules.

Here the lack of experimental data do not allow us to conclude on the presence of a threshold concentration after which $\kappa$ is a constant, linking the scope of this analysis. The collection of more data points is currently in progress.

Interestingly the value of $\kappa$ in the case of salt concentration $C_{\text {ion }}=100 \mathrm{~mol} \cdot \mathrm{m}^{-3}$ is close to the one found for the neutral macromolecule PEG-PFPE. These two equilibrium also share the same values of $\Gamma_{\infty}$. PEG-PFPE is neutral molecule, and therefore no dependence on the electrolyte concentration is expected. Thus the comparable values of the $\kappa$ might be an indication that the PFPE chain starts behaves as a neutral molecule, due to the screening of the electrostatic interactions, and that the plateau region described above is already reached.

The following section will be focused on the study of dynamic of adsorption for Krytox-H and $\mathrm{Krytox}-\mathrm{NH}_{4}$.

\subsubsection{Kinetics of adsorption.}

In this part we want to characterize the mode of adsorption of the two molecules. We have already mentioned in chapter 3 that the potential barrier created by the presence of charges at the interface provokes a transition from diffusion-limited adsorption to transfer-limited adsorption [26]. With this analysis we would like to address the possible interactions of the counter ion with this electrostatic barrier. For this we consider the kinetics of adsorption of the two molecules Krytox-H and Krytox- $\mathrm{NH}_{4}$ at the interface. The method used is identical to the one applied for PEG-PFPE molecule in the previous section.

\section{Krytox-H: effect of the dimerization}

We start the study with the molecule Krytox-H. In figure 6.9 are presented the extracted characteristic time for the early kinetics (panels (a) et (b)) and for the late kinetics (panels (c) et (d)). We compare the two cases of millipore water and brine as the aqueous phase. For each 
$\mathrm{H}_{2} \mathrm{O}+\mathrm{NaCl} 10^{-4} \mathrm{~mol} \cdot \mathrm{m}^{-3}$
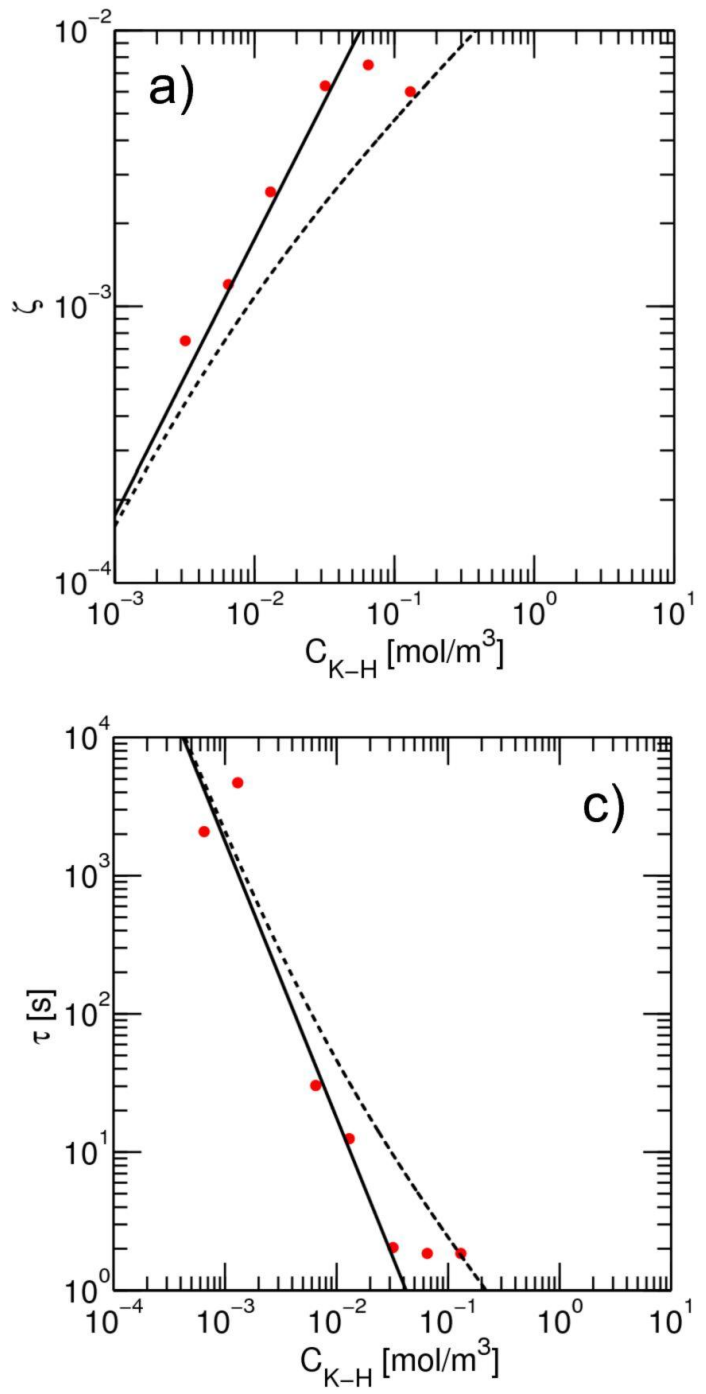

$\mathrm{H}_{2} \mathrm{O}$
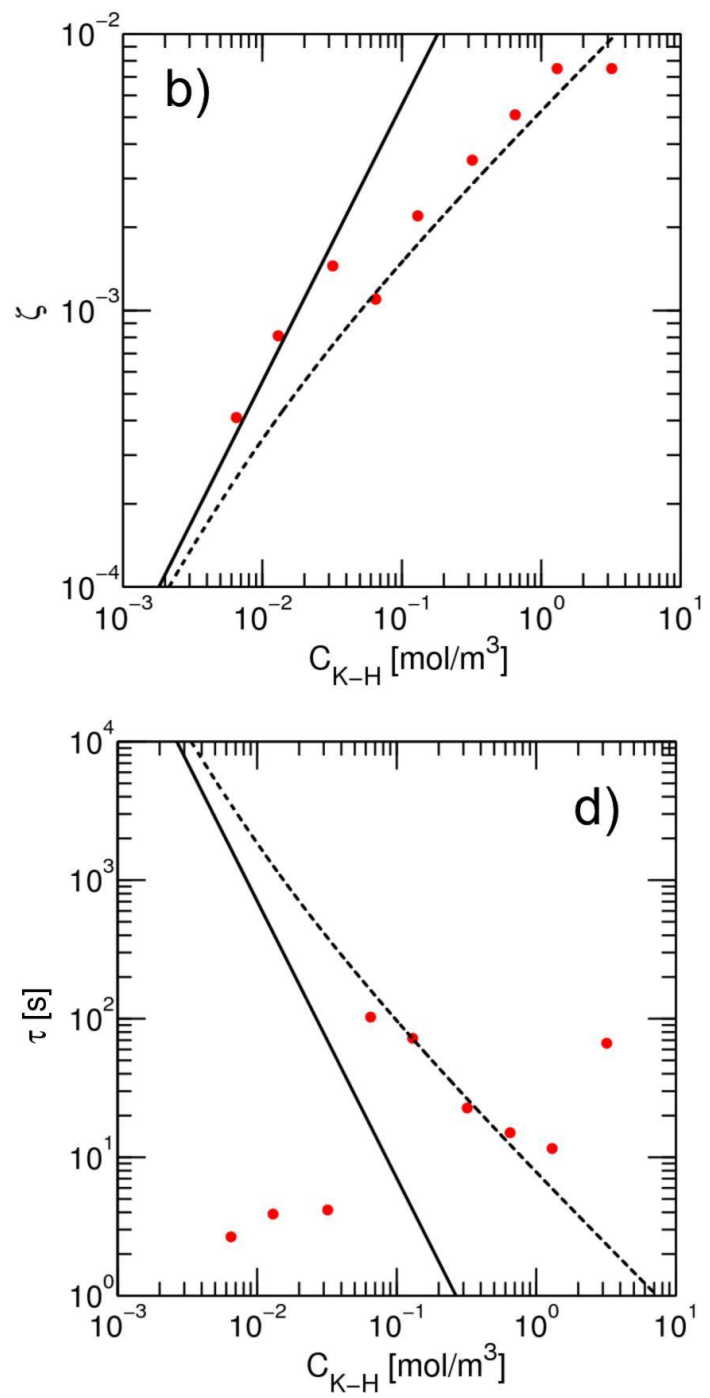

Figure 6.9 Characteristic time scale of the adsorption kinetics of the molecule Krytox-H to the interface. For the early kinetics; $(\zeta)$ for systems: (a) oil / millipore water and (b) oil/ Salty water. For the late kinetics; $(\tau)$ for system (c) oil / millipore water and (d) oil / salty water. The solid line and the dashed line represent the scalings for respectively: the diffusion in absence of dimers (equations 6.4 and 6.2), and in presence of dimers (equations 6.12). The range of concentration is situated below the CMC. From these different cases we extract a diffusion constant for PFPE chain $D \sim 10^{-10} \mathrm{~m}^{2} \cdot \mathrm{s}^{-1}$ and a reaction constant for the dimerization process equal to $K_{\mathrm{d}}=50 \mathrm{~m}^{3} \cdot \mathrm{mol}^{-1}$.

cases we compare the characteristic times with the the scalings for the diffusion of monomers, with the equations 6.3 and 6.2 .

We first notice that in the range of concentration below $C_{\mathrm{K}-\mathrm{H}} \sim 3 \times 10^{-2} \mathrm{~mol} \cdot \mathrm{m}^{-3}$, the 
characteristic time extracted are consistent with a pure diffusion model. See figure 6.9 (a) and (c). Above this concentration, the characteristic times are deviating from this model as shown in figure 6.9 (b) and (d).

It has been demonstrated that the presence of the $\mathrm{H}^{+}$ion allow the formation of a hydrogen bond between $\mathrm{OH}$ groups on two different molecules[76, 77]. See figure 6.10. Therefore at equilibrium in the bulk phase a certain part of the Krytox-H monomer population will react and form dimers. The aggregation processes is described as a reversible reaction of dimerization / de-dimerization. The proportion of dimers is likely to have an effect on the adsorption kinetics of surfactant at the interface.

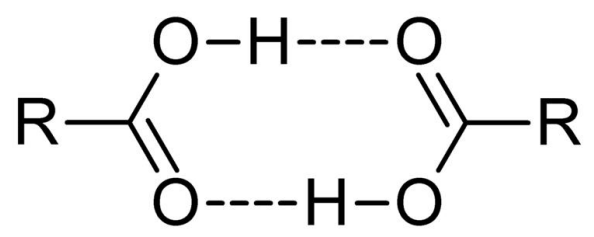

Figure 6.10 Example of a dimer stabilized by H-bonds between $\mathrm{OH}$ group. In the case of Krytox$\mathrm{H}$, the $\mathrm{R}$ corresponds to the PFPE chain. This aggregates is found to coexists in solvent with monomers, and to change significantly the dynamic of adsorption of Krytox-H to the interface[76].

We derive here the scaling laws relative to the diffusion of dimer to the interface. We propose a model where the kinetics of dimerization is described from the aggregation theory. For a dimer, the dynamic equilibrium is written as:

$$
X_{1}+X_{1} \rightleftharpoons X_{2}
$$

where $X_{1}$ is a monomer and $X_{2}$ a dimer. The equilibrium of this reaction is determined by the reaction constant $K_{\mathrm{d}}$. The initial amount of monomer surfactant $C_{\mathrm{K}-\mathrm{H}}$ dissolved into the solvent is partitioned in between these two states, with respective concentrations of monomer $C_{1}$ and concentration of dimers $C_{2}$. The the mass conservation predicts:

$$
C_{\mathrm{K}-\mathrm{H}}=C_{1}+2 C_{2}
$$

And the equilibrium concentrations are given by:

$$
C_{1}^{2} K_{\mathrm{d}}=C_{2}
$$


The solution of these two equation gives the following scaling.

$$
C_{1}=-\frac{1}{4 K_{\mathrm{d}}}+\frac{\sqrt{8 C_{\mathrm{K}-\mathrm{H}} K_{\mathrm{d}}+1}}{4 K_{\mathrm{d}}}
$$

We assume in our model that the dimers do not adsorb directly to the interface, but first decay into monomers that will adsorb to the interface. This reasoning follows the one exposed in chapter 3 about the two mechanisms in which of micelles can directly adsorb to the interface. Based on this assumption, we derive a model able to describe the evolution of the typical time scales of adsorption for the early and the late kinetics, based on the scaling derived from the Ward and Tordai equation, and taking into account the proportion of monomers that are not used to form dimers. The early characteristic time $\zeta$ and the late characteristic time $\tau$ should then respectively take the following polynomials form in $\mathrm{C}_{\mathrm{K}-\mathrm{H}}$ :

$$
\begin{aligned}
& \zeta^{\prime}=2 n R T \sqrt{\frac{D}{\pi}}\left[-\frac{1}{4 K_{\mathrm{d}}}+\frac{\sqrt{8 C_{\mathrm{K}-\mathrm{H}} K_{\mathrm{d}}+1}}{4 K_{\mathrm{d}}}\right] \\
& \text { and } \\
& \tau^{\prime}=\frac{\pi}{4 D}\left[\frac{4 \Gamma_{\infty} K_{\mathrm{d}}}{\sqrt{8 K_{\mathrm{d}} C_{\mathrm{K}-\mathrm{H}}}-1}\right]^{2}
\end{aligned}
$$

This model does not takes into account the de-dimerization kinetics of the dimers that could occur during the depletion of the monomers in the subsurface. Therefore the two scalings of diffusion with and without dimerization represent the limiting cases of the processes that we study.

Two variables have to be determined in the model: the diffusion constant of the molecule $D$, and the reaction constant of the dimerization reaction $K_{\mathrm{d}}$. On figure 6.9, the scaling predicted by the equation 6.12 is materialized by dashed lines. The deviation noticed above $C_{\mathrm{K}-\mathrm{H}} \sim 3 \times 10^{-2} \mathrm{~mol} \cdot \mathrm{m}^{-3}$ is well fitted with our model

Quantitatively, we are able to discriminate a critical concentration where the cross-over between the monomer and the dimer regime occurs. This cross-over occurs for a initial surfactant concentration of $C_{\text {crit }} \sim 3 \times 10^{-2} \mathrm{~mol} \cdot \mathrm{m}^{-3}$.

For the concentration below $C_{\text {crit }}$ we find a diffusion coefficient of approximatively $D \sim$ $10^{-10} \mathrm{~m}^{2} \cdot \mathrm{s}^{-1}$. This value is in good agreements with other measurements made using fluorescence confocal spectroscopy which measured values around $D \sim 10^{-10} \mathrm{~m}^{2} \cdot \mathrm{s}^{-1}[78]$. Applying 


\begin{tabular}{ccccc}
\hline Parameter & \multicolumn{2}{c}{ Krytox-H } & \multicolumn{2}{c}{ Krytox-NH${ }_{4}$} \\
\hline \hline & $\mathrm{H}_{2} 0$ & $\mathrm{NaCl}$ & $\mathrm{H}_{2} 0$ & $\mathrm{NaCl}$ \\
\hline $\mathrm{D}\left[\mathrm{m}^{2} \cdot \mathrm{s}^{-1}\right]($ Early $)$ & $4 \times 10^{-9}$ & $1 \times 10^{-10}$ & $4 \times 10^{-9}$ & $5 \times 10^{-10}$ \\
\hline $\mathrm{D}\left[\mathrm{m}^{2} \cdot \mathrm{s}^{-1}\right]($ Late $)$ & $10^{-9}$ & $1 \times 10^{-10}$ & $\emptyset$ & $\emptyset$ \\
\hline$K_{\mathrm{d}}\left[\mathrm{m}^{3} \cdot \mathrm{mol}^{-1}\right]$ & 50 & $\emptyset$ & $\emptyset$ & $\emptyset$ \\
\hline
\end{tabular}

Table 6.3 This table gathers the kinetic properties of the surfactants Krytox-H and Krytox- $\mathrm{NH}_{4}$. The diffusion coefficients $D$ of the monomer R-X, are extracted from the fitting of the kinetic model for monomers (equations 6.4 and 6.2), and dimers diffusion (equation 6.12). If applicable we also use the dimer model to estimate the reaction constant of the dimerization process $K_{\mathrm{d}}$. The values found form a consistent panel, in good agreement with other measurements performed using fluorescence microscopy[78].

the Stokes-Einstein relation (equation 6.13) to this value we find a typical molecular dimension of the order of:

$$
R=\frac{k_{\mathrm{b}} T}{6 \pi \eta D} \sim 2 \mathrm{~nm}
$$

with $k_{\mathrm{b}}$ being the Boltzmann's constant. This size is acceptable for a simple PFPE chain like Krytox-H.

Above the $C_{\text {crit }}$ the mode 1 developed above allows us to estimate a value of $K_{\mathrm{d}}$. To fit the model, first the diffusion coefficient is set to $D \sim 10^{-10} \mathrm{~m}^{2} \cdot \mathrm{s}^{-1}$, which allows us to retrieve a reaction constant $K_{\mathrm{d}}=50 \mathrm{~m}^{3} \cdot \mathrm{mol}^{-1}$. To estimate the values of this parameter we can calculate the corresponding dissociation enthalpy given by the Gibbs relationship:

$$
\Delta G_{\mathrm{d}}=-R T \ln K_{\mathrm{d}} \sim-10 \mathrm{~kJ} \cdot \mathrm{mol}^{-1}
$$

This value is consistent with the strength of hydrogen bonds predicted for dimers of carboxylic acids[79]. We can then estimate the relative proportion of dimers to bulk concentration present in the continuous phase necessary to have a noticeable effect on the adsorption, $\left.C_{2}\right|_{\mathrm{C}_{\text {crit }}} \sim 25 \%$. The values extracted for the different cases are gathered in table 6.3 , for easy comparison.

\section{Krytox-NH}

We have seen that the adsorption of Krytox-H molecule can not be understood without taking into account the process of dimerization. We are now interested to compare the kinetics of Krytox-H with the case of Krytox- $\mathrm{NH}_{4}$ that does not form such aggregates. The presence 

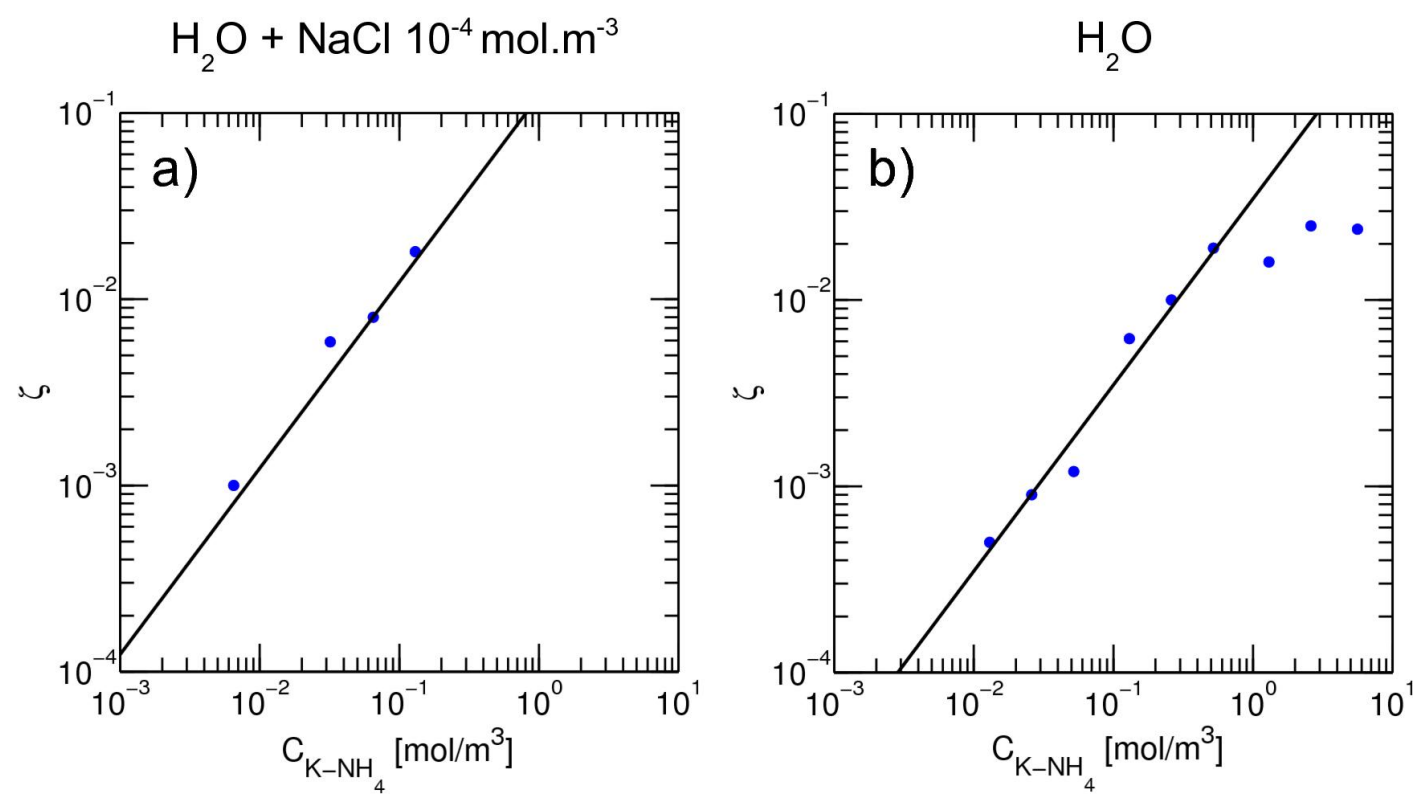

Figure 6.11 Characteristic time scale of the adsorption kinetics of the molecule Krytox-H to the interface. For the early kinetics; ( $\zeta$ ) for systems: (a) oil / millipore water and (b) oil/ Salty water. The best fit where found for a diffusion constant of the PFPE chain around $D \sim 10^{-10}-10^{-9} \mathrm{~m}^{2} \cdot \mathrm{s}^{-1}$. The presence of the bounce in the dynamic of surface tension does not allow us to conclude on the late kinetics for this surfactant.

of the $\mathrm{NH}_{4}^{+}$ion in the Krytox- $\mathrm{NH}_{4}$ molecules prevents the formation of dimers in the bulk. Therefore, the kinetics of this molecule correspond to a case where the diffusion alone of the monomer can be analyzed. The results of this kinetic analysis are presented on figure 6.11. In this figure the solid line represents the diffusion model corresponding to the equation 6.2 , We see that the model describes well the characteristic time extracted from a large range of surfactant concentrations. The only deviation from the model occurs for concentrations above the $\mathrm{CMC}$, and can be attributed, similarly to the PEG-PFPE molecule, to the the presence of micelles. The fit allows us to extract a diffusive constant $D \sim 10^{-10} \mathrm{~m}^{2} \cdot \mathrm{s}^{-1}$. This value is in very good agreement with the values extracted for the Krytox-H monomers, and corresponds to a realistic size of molecules, as previously calculated with equation 6.13. The study of this additional case confirms that the deviations observed for Krytox-H can be attributed to the dimerization process.

The presence of the "bounce" in the dynamic surface tension, prevents us from making a satisfying analysis of the late kinetics. However, we would like to discuss here an additional effect that may be responsible for this feature. As described above the molecules at the interface are in dynamic equilibrium, which implies that constant adsorption / desorption events 
are occurring, even when the equilibrium surface tension is reached. The desorption can not occur without the association between a PFPE chain at the interface and an ion from the bulk. We demonstrated that this process is closely linked to the electrolyte concentration. In the case of millipore water, generally with a $\mathrm{pH} \sim 5-6$ (due to the rapid dissolution of carbon dioxide from the air), the concentration of $\mathrm{H}^{+}$ions can be estimated to $C_{\mathrm{H}^{+}} \sim 10^{-3}-10^{-2} \mathrm{~mol} \cdot \mathrm{m}^{-3}$. In comparison, the concentration of $\mathrm{NH}_{4}^{+}$ion in the water phase can be estimated at the early times of the kinetics by the maximum surfactant coverage of the interface. The number of molecules at maximum coverage, in absence of salt, the theoretical maximum concentration of ion $\mathrm{NH}_{4}^{+}$is given by:

$$
\left.C_{\mathrm{NH}_{4}^{+}}\right|_{\max }=\frac{\Gamma_{\infty} S}{V} \sim 10^{-3} \mathrm{~mol} \cdot \mathrm{m}^{-3}
$$

Where is $S$ the surface area of the droplet extracted from the fit of the pendant drop shape and $V$ is the droplet volume. This theoretical concentration is slightly smaller than $\mathrm{C}_{\mathrm{H}^{+}}$. Therefore in the process of desorption, the PFPE chain will be more likely to associate with $\mathrm{a}^{+}$ion. This event is equivalent to the desorption of a Krytox-H molecule into the subsurface. Depending on the characteristic time of desorption, the exchange of ions might be rapid enough to induce a local concentration of Krytox-H in the subsurface high enough to allow the production of dimers. This complex kinetics could well explain the presence of the bounce. In addition, in the presence of a salt concentration of $\mathrm{C}_{\mathrm{Na}^{+}}=10^{2} \mathrm{~mol} \cdot \mathrm{m}^{-3}$ far above $\mathrm{C}_{\mathrm{H}^{+}}$and $\mathrm{C}_{\mathrm{NH}_{4}^{+}}$the bounce is not visible anymore. Following the same reasoning, it is very likely that the molecules desorbed from the interface are a new type of molecule PFPE $+\mathrm{Na}^{+}$, that is not subject to dimerization, as $\mathrm{Na}^{+}$being monovalent.

To full answer this question, an extensive study is required in order to eliminate other effects such as the reaching of a mesoequilibrium described by Hua et al. that is also found to depend on the electrolyte concentration [80].

It is interesting to note that the chemically close structure of Krytox-H and Krytox- $\mathrm{NH}_{4}$ have global adsorption behaviors that are completely different. We have seen that one reason is the ability of the Krytox-H to form dimers in the solution.

To sum up the analysis of the kinetics in its essential features:

- As expected in pendant drop method the kinetic of adsorption of overall neutral particles can be described a diffusion-limited in the asymptotic case $t \rightarrow 0$, and the for late kinetics $t \rightarrow \infty$. 
- In the specific case of the dimerization of the surfactant molecules in the bulk phase the diffusion-limited regime is also recovered both for the early and late kinetics. A model depending on the properties of the dimerization reaction describes quantitatively the adsorption of the remaining monomers.

- From the point of view of the dynamics of adsorption, the presence of electrolytes in the water phase does not have a noticeable effect on the diffusion of monomers to the interface. However, they do influence the equilibrium repartition of the PFPE charges at the interface by the screening of the electrostatic interactions.

\subsection{Dynamic Light Scattering: Micellarization processes}

The characterization of dispersed colloidal particles in a solution can be characterized by the Dynamic Light Scattering (DLS) method, as described in the chapter 5. Applied to surfactant molecules in different concentrations, the DLS method allows for the determination of the number and the size of the micelles present in solution. Usually the signal is extracted from a range of different concentrations. When the surfactant exists in solution as a monomer only, the DLS signal is too weak to detect the molecule. Above the CMC the scattering signal coming from the micelles can be detected from the surrounding noise, if the micelles exist in a sufficient number, and are large enough to be detected. The scattering signal usually allows for the quantitative measurement of the size and amount of the micelles.

\subsubsection{Macromolecule PEG-PFPE}

The figure 6.12 shows the result of DLS analysis of the surfactant solutions in HFE. Above the $\mathrm{CMC} C=1.5 \times 10^{-1} \mathrm{~mol} \cdot \mathrm{m}^{-3}$ the scattering signal is increasing and micelle sizes of $\sim 100 \mathrm{~nm}$ is found. This measurement is consistent with the pendant drop determination of the CMC, and other measurement performed with similar molecules[71].

\subsubsection{PFPE chains}

The DLS signals for Krytox-H and Krytox $-\mathrm{NH}_{4}$ are too small to detect the CMC, even for concentration above $2 \mathrm{~mol} \cdot \mathrm{m}^{-3}$ where the $\mathrm{CMC}$ is detected from the Langmuir isotherm. The smallest limit resolution in size of our apparatus is of the order of $\sim 5 \mathrm{~nm}$. This indicates 


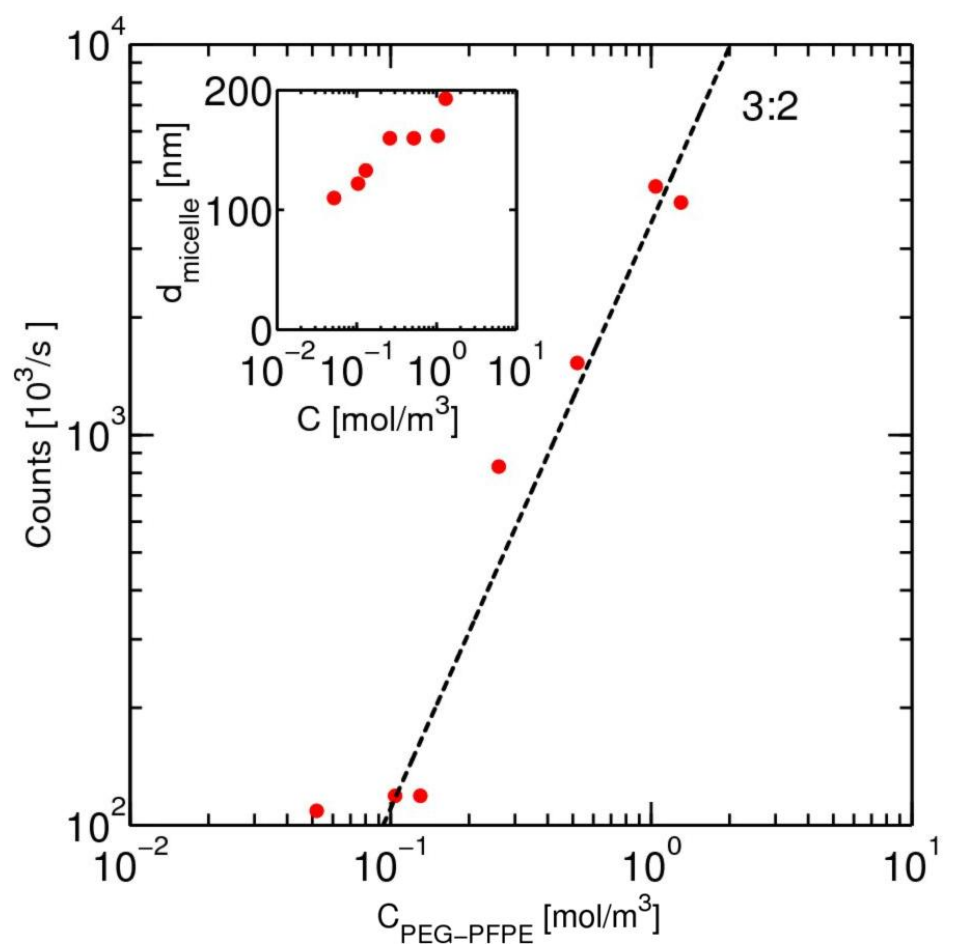

Figure 6.12 DLS measurements for the surfactant between concentrations $C=5.2 \times 10^{-3}$ and $1.3 \mathrm{~mol} \cdot \mathrm{m}^{-3}$. The solid black line provides a CMC measurement consistent with the pendant drop method. Above the CMC, the scattering signal increases non-linearly. Inset: The micellar aggregates present in the solution above the CMC have a typical size of 100 $\mathrm{nm}$. The small variation of the micelle size with the surfactant concentration accounts for the non linear behavior observed on the scattering intensity beyond the CMC.

that the micelles size for both neutralized PFPE chains are smaller than this value, and in sufficiently small amount not to be conveniently detected with this method. 


\subsection{Conclusion}

The three molecules analyzed in this chapter, allowed us to browse a large spectrum of surfactant properties that can be featured in dynamic tensiometry: the determination of the adsorption mode to the interface and the associated kinetics parameters. This analysis allowed us also to characterize the properties of the adsorption equilibrium. We could then conclude on the repartition of the surfactant at the interface, based upon the interactions between the molecules.

We compared the case of a macromolecule PEG-PFPE to the cases of two smaller molecules Krytox-H and Krytox- $\mathrm{NH}_{4}$ of the same family. We have seen that the mode of adsorption at the interface is mainly diffusion limited. We determined from the diffusion coefficients $D$ of the molecules.

We have shown from the dynamics of Krytox-H that the presence of a dimerization in the bulk phase can be elucidated by both the early and the late surfactant adsorption kinetics. The measurement were in good agreement with the simple model that has been derived in this chapter, taking into account the partition of the initial concentration of the surfactant molecules in the bulk into a monomer and a dimer population. Using this model we were able to deduce the value of the hydrogen bond energy within the dimer, consistent with the one predicted in the literature. The purely diffusive behavior measured for the molecule Krytox- $\mathrm{NH}_{4}$ that does not produce dimers, confirmed this analysis.

The molecule Krytox-H has an interesting other feature which is that the $\mathrm{H}^{+}$ion release in the aqueous phase contributes to the acidification of the aqueous phase. Given the number of species adsorbed at the interface, this effect is small for millimetric droplets. The influence becomes more important however when the adsorption occurs for micrometric droplets where the surface area to volume ratio is higher. Such as the case for droplets in microchannels. Therefore the presence of a $\mathrm{pH}$ sensitive marker, such as a fluorescent dye[81] or a precipitating species allows the direct reading of the amount of surfactant molecules that have released a $\mathrm{H}^{+}$into the volume of the droplet.

The panel of surfactants used in this study is representative of the degree of complexity possible for the kinetics of surfactants adsorption to the interface. We have seen that in the case of the PEG-PFPE surfactant, the measurement is extremely sensitive to the presence of uncontrolled convective transport and the curvature of the interface. This effects can profoundly influence the dynamics of the adsorption. This constitutes one the main reasons motivating us to develop a new microfluidic method. Here the emulsification method allow us 
to produce a large amount of droplets with identical sizes and compositions. In addition, the flow profile around the droplet can be measured precisely and therefore its influence on the adsorption can be predicted.

From a practical point of view, the PEG-PFPE surfactant presents a general behavior that is well described by the diffusive model over a large range of concentrations. Furthermore the DLS analysis has allowed us to perform quantitative measurements of the structure of the micelles. The knowledge of the is surfactant is a solid reference point for our analysis in microfluidics. In the following chapter we describe a method for the dynamic surface tension measurement on chip. 


\section{Microfluidic Dynamic Interfacial Tensiometry: $\mu$ DIT}

Parts of this work have been published in the article "Microfluidic Dynamic Interfacial Tensiometry: $\mu D I T ”$, Soft Matter, 2014

\subsection{Introduction}

In the previous chapter we have seen that a precise dynamic tensiometry measurement of one surfactant, allows a direct quantification of the molecule kinetic parameters. However measurements using pendant drop method can be subject to uncontrolled convective flows that make the measurement imprecise. We have seen that the early kinetics of the surfactant adsorption is particularly sensitive to this disturbances. Performing dynamic tensiometry measurements in a different environment where the flux around the interface are controlled would be a solution to circumvent these side effects. This is possible in microfluidics where the flow conditions can be measured with great precision and the production of calibrated emulsions is well-established. In this chapter we present a new microfluidic method for the measurement of surfactant adsorption kinetics via interfacial tensiometry on a microfluidic chip. The principle of the measurement is based on the deformability of droplets as a response to hy- 
drodynamic forcing through a series of microfluidic expansions. We focus our analysis on one perfluorosurfactant molecules, of practical interest for droplet-based microfluidic applications. We show that although the adsorption kinetics is much faster than the kinetics of the corresponding pendant drop experiment, our droplet-based microfluidic system has a sufficient time resolution to obtain quantitative measurements at the sub-second time-scale on nanoliter droplet volumes, leading to both a gain in a factor of $\sim 10$ in time resolution and a downscaling of the measurement volumes by a factor $\sim 1000$ compared to standard techniques. Our approach provides new insights in the adsorption of surfactant molecules at liquid-liquid interfaces in confined environment, relevant to emulsification, encapsulation and foaming, and the ability to measure adsorption and desorption rate constants.

Amphiphilic molecules are ubiquitous in our daily lives, widely represented in Nature [82, 83] and intensively used in the pharmaceutical, cosmetic, food and petroleum industries, [84, 85]. Surfactant molecules are used as detergents and dispersing materials, coating and foaming agents, emulsifiers and biocides, spanning a wide range of functions [50, 86, 87].

More than two hundred years after the first observations reported by Benjamin Franklin of the damping of waves on a lake by fatty acids [88], understanding the dynamics of surfactant films remains a challenge, both theoretically and experimentally. Interfacial rheology and adsorption dynamics are however key concepts in understanding foaming, emulsification and encapsulation processes [89, 90] as well as dynamic properties of biological membranes [91]. Theoretically, understanding the dynamics of the surfactant requires an accurate description of the bulk molecular self-assembly, of the transport towards the interface and of the bulk - interface equilibrium [14, 29, 34, 92]. The latter point relies on proper modeling of the surfactant interactions, especially in the case of ionic species [26, 93] or of the interfacial reorganisation for oligomers and polymers [94, 95]. Experimentally, only a few methods enable a sensitive and direct measurement of the organization of molecular monolayers at time-scales where the properties of the interface are influenced by the molecular self-assembly [34, 96]. Tensiometry is widely used for both equilibrium and dynamic measurements but provides limited information on adsorption-desorption processes due to the predominance of diffusion at large time scales [29, 75, 97, 98]. Deviations of the experimental data from the minimal diffusive-limited model introduced by Ward and Tordai are almost systematically observed, and attributed to curvature effects [99--102], convective currents [75, 103], or adsorption barriers [14, 26]. These adsorption barriers are revealed in the transfer-limited regimes which dominate at small dimensions [25, 97] and in convective systems [104, 105], namely in the conditions relevant to emulsification or foaming. We address here the dynamics of surfactant 
adsorption in the context of microfluidic emulsification.

Microfluidics offers controls for the manipulation of multiphase systems in confined environments. The possibility to measure transient states at short timescales $(\sim \mathrm{ms})$ makes microfluidics especially appealing as a new tool to quantitatively study the physics and physicalchemistry of interfaces and emulsions [42, 51, 52, 71, 74, 106-108]. Microfluidic systems have been designed for interfacial tension measurements, for example making use of the deformation of a droplet entering a constriction [109]. In the limit of the spheroid shapes, the surface tension is obtained through the analysis of the rate of deformation and the velocity variation in the constriction [109-111]. This type of analysis has further been used to obtain dynamic information on the interfacial properties of butanol with a time resolution of the order of one second [104]. The dynamic processes of adsorption can influence emulsification at a much smaller time-scale [31, 74, 112, 113], down to the limit of capillary breakup [114]. To date, a direct quantitative measurement of dynamic interfacial properties at the subsecond time-scale in presence of surfactants is still lacking. In this chapter we present a method usable to measure dynamic surface tension of droplet, with a resolution down to the millisecond. This method is not restricted to small droplet deformation and does not rely on assumption of specific droplet shapes during deformations. It is applied for the first time to the characterization of the adsorption properties of a block copolymer perfluorosurfactant of practical interest for biotechnology applications in microfluidics [73, 115]. We show for this surfactant that in flow conditions the adsorption is limited by the equilibrium between the bulk phase and the interface. In addition the dynamics of adsorption in the microfluidic case, exposed to convection are transfer-limited. This regime differs from the pendant drop regime where convective processes are largely dominated by diffusion of the perfluorosurfactant molecule from the bulk. Our microfluidic approach provides a new tool to determine the kinetic properties such as the adsorption and desorption rate constants from the interface. It provides a new tool to determine the kinetic properties such as the adsorption and desorption rate constants from the interface.

This chapter will be divided as follow. In a first section we will introduce and motivate the choice of the microfluidics geometry used for the analysis. In a second section we will calibrate the surface tension of an interface to the deformation of a droplet entering a sudden planar expansion. Thereafter we will apply the microfluidic method to the description of the PEG-PFPE molecule, characterized in the previous chapter. We will compare the kinetic properties and equilibrium state of the molecule to the results obtained by pendant drop method, 
before a discussion of the results.

\subsection{Microfluidic geometry}

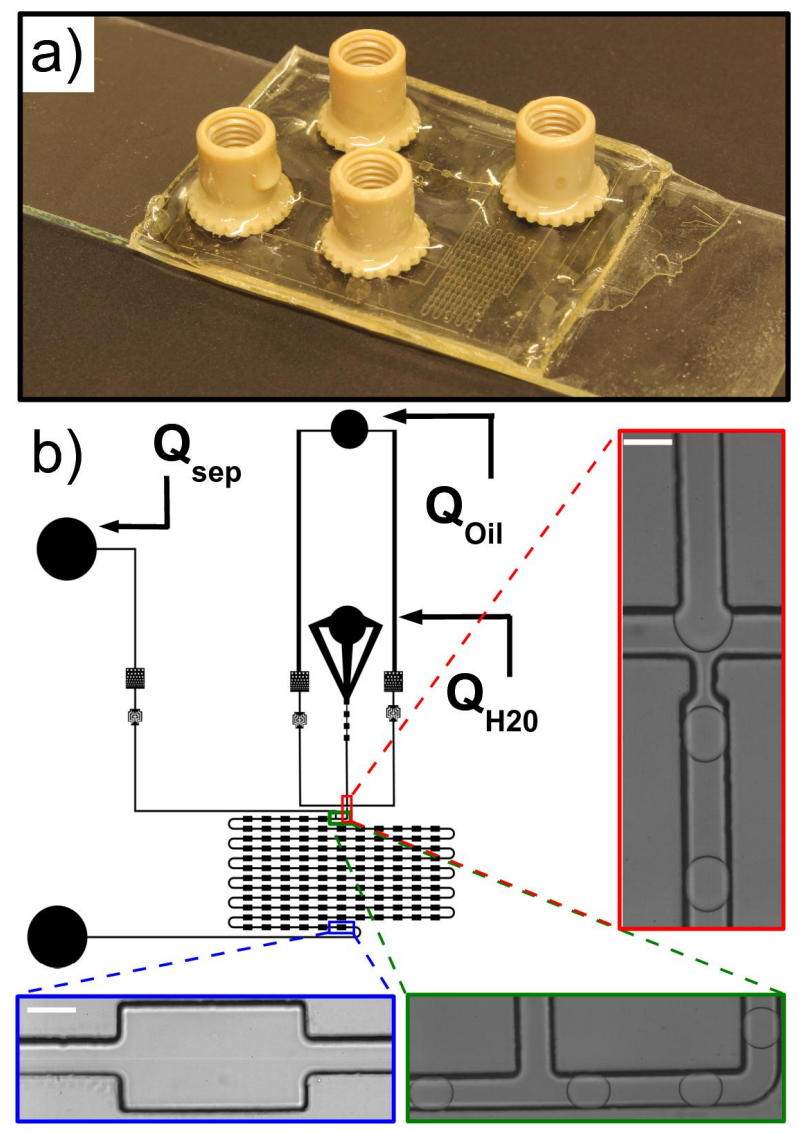

Figure 7.1 (a) Photography of the macroscopic aspect of the NOA chip. The typical base-diameter of the connectors is $0.85 \mathrm{~cm}$. (b) Geometry of the microfluidic channel used for on-chip Dynamic Interfacial Tensiometry $(\mu \mathrm{DIT})$. Droplets are produced by focusing a stream of water $Q_{\mathrm{H} 2 \mathrm{O}}$ in a stream of fluorinated oil / surfactant solution $Q_{\mathrm{oil}}$ (red enlarged area) and spaced by a side stream of the same oil solution $Q_{\text {sep }}$ (green enlarged area). The droplets are flowed through in series of expansions (blue enlarged area) where the droplet deformation profile is recorded and interfacial properties analyzed. A series of expansions regularly distributed along a delay line is used to access the dynamics of the adsorption process during flow. The white scale bar has a length of $100 \mu \mathrm{m}$.

The present study is based on the simple idea, that an interface deformation can be directly quantified only by one parameter that is the surface tension. Monodisperse droplets are produced at a standard flow focusing junction [43]. We designed an additional control on droplet 
velocity and spacing independent of the droplet production using a side oil flow rate $Q_{\text {sep }}$. displayed in figure 9.2 (a). In our experiment the deformation is induced by the flowing of a droplet through a sudden planar expansion with ratio 3:1. Afterwards the droplet is allowed to relax through a chamber. This unit is designed in a such a way that distributed along a delay line allows the study of a droplet deformation and relaxation, as well as retaining high time resolution. In accordance to our experimental constraints, we designed the functional part of the device, depicted in figure 9.2 (b) consists in a delay line with 121 successive chambers $W_{2} 300 \mu \mathrm{m}$ wide, $L=500 \mu \mathrm{m}$ long, each of them connected by a channel $W_{1} 100 \mu \mathrm{m}$ wide with length $L$. The length $L$ allows us to monitor the droplet a droplet free relaxation, that is to say that the deformed droplet can relax to a spherical shape without feeling the influence of the constriction. 

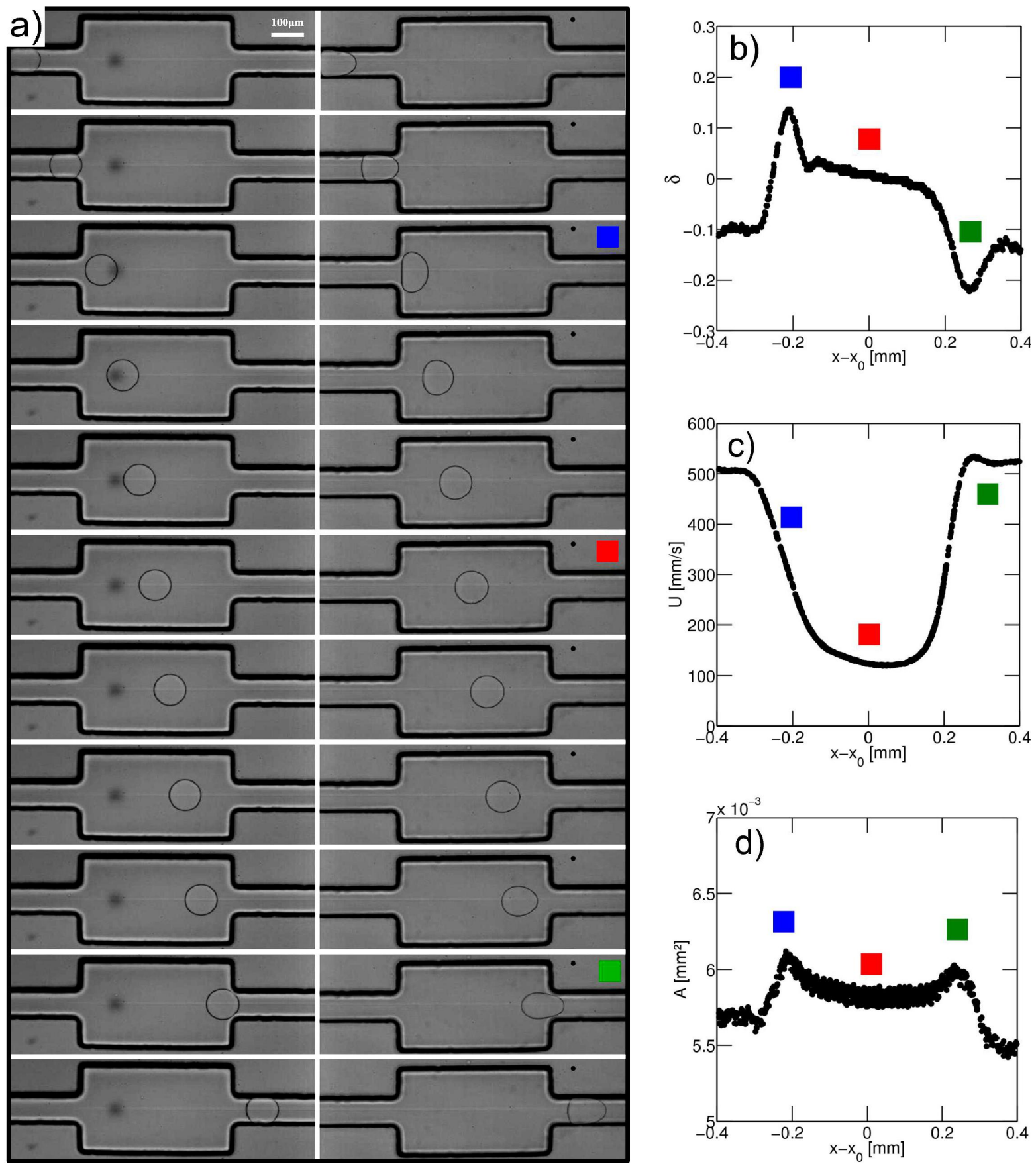

Figure 7.2 (a) Successive micrographs of a droplet deformation while passing through our geometry. The time between two successive image is $0.4 \mathrm{~ms}$. Two surface tensions are used: $\gamma=$ $49.5 \mathrm{mN} \cdot \mathrm{m}^{-1}$ on the left column, and $\gamma \sim 2 \mathrm{mN} \cdot \mathrm{m}^{-1}$ on the right column. Three important positions for our study are indicated: the droplet maximum deformation ( $\mathbf{\square})$; in the middle of the chamber when relaxed to a sphere $(\square)$; entering the constriction $(\square)$. These three points are reported with the same color code on the deformation profile (b), the speed profile (c), and the area profile (d). 


\subsection{Droplet deformation at a microfluidic expansion}

In a first series of experiments, we consider the case of solutions without surfactant. We produce droplets at a frequency of $\sim 100 \mathrm{~Hz}$. For each expansion, the droplet is deformed in the direction perpendicular to the flow, with a maximum deformation $\delta_{\max }$ close to the entrance of the expansion (figure 7.2), as previously observed [109]. For a fixed channel geometry, $\delta_{\max }$ is a function of the physical quantities (i) the droplet velocity $U_{\text {drop }}$, (ii) the interfacial tension $\gamma$ and (iii) the droplet radius $R$. The viscosities of both phases also control the deformation. In all experiments, the viscosity of the continuous phase is kept constant to $1.24 \mathrm{mPa} \cdot \mathrm{s}^{-1}$ and it we found that the viscosity of the dispersed phase is not a relevant parameter, at least for the viscosities ranging from 1 to $10.8 \mathrm{mPa} \cdot \mathrm{s}^{-1}$, see figure 7.3 .

We use a mixture of glycerol/water to achieve a range of viscosity of the disperse phase from $\eta_{\text {in }}=1 \mathrm{mPa} \cdot \mathrm{s}$ to $\eta_{\text {in }}=22.5 \mathrm{mPa} \cdot \mathrm{s}$ at $20{ }^{\circ} \mathrm{C}$. The kinetics of the droplet deformation with a fixed amount of surfactant, show no dependence on dispersed phase viscosity ranging up to $\eta_{\text {in }}=10.8 \mathrm{mPa} \cdot \mathrm{s}$. Beyond this viscosity a weak variation is observed, as shown in figure 7.3 .

This result is consistent with the one obtained by Taylor showing that the deformation of sheared droplets is mainly controlled by the viscosity of the external fluid [110].

From the set of remaining physical parameters, two dimensionless numbers are expected to control the problem, (i) the Capillary Number calculated using the viscosity of the continuous phase $C a=\eta U_{\text {drop }} / \gamma$ and (ii) a geometrical number $R^{*}=2 R / W_{1}$ that relates the droplet radius $R$ to a typical length, taken here as the channel width $W_{1}$. Looking for a scaling relationship based on these two numbers, we first calibrate the dependence of the deformation on the speed and interfacial tension of the droplet. For a fixed droplet size, we vary the oil flow rate $Q_{\text {sep }}$ in the side injection channel. The maximal droplet deformation is measured as a function of the flow velocity. For water in oil $\left(\gamma=50 \mathrm{mN} \cdot \mathrm{m}^{-1}\right)$, we observe that the deformation increases for increasing velocities, in qualitative agreement with the previous experiments and theoretical expectations [109,111]. The surface tension is then varied by adding ethanol in the dispersed aqueous phase that does not modify significantly the viscosity ratio between the continuous and the dispersed phase (figure 7.6). All data collapse on a master curve when using the Capillary number. This indicates that the Capillary Number is the relevant dimensionless number of the problem, and a power law relationship $\delta_{\max } \sim C a^{2 / 3}$ describes the scaling. 


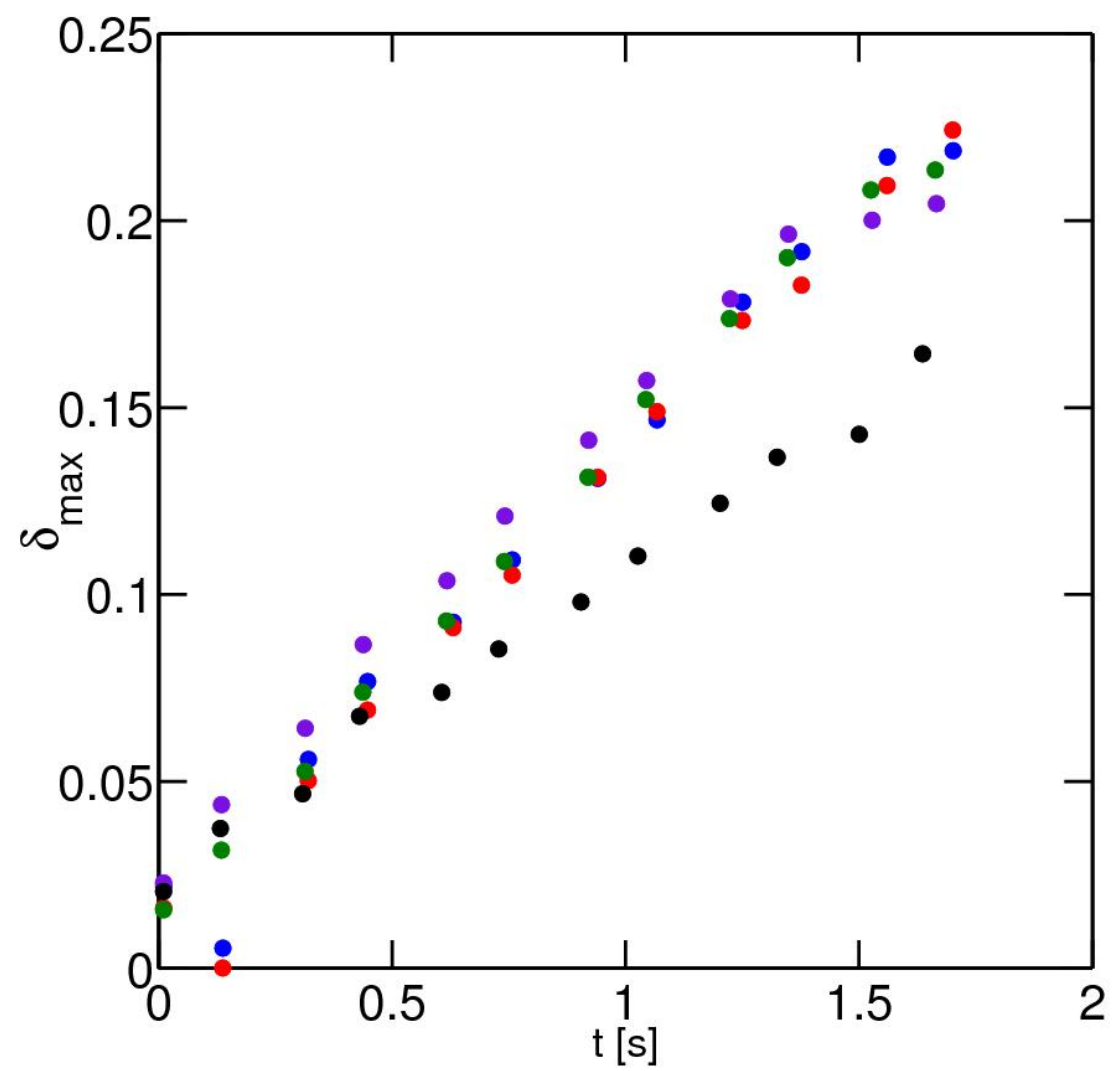

Figure 7.3 Dependence of the droplet maximum deformation on disperse phase viscosity: $\eta_{\text {in }}=$ $1.00 \mathrm{mPa} \cdot \mathrm{s},(\bullet) ; \eta_{\text {in }}=1.76 \mathrm{mPa} \cdot \mathrm{s},(\bullet) ; \eta_{\text {in }}=3.72 \mathrm{mPa} \cdot \mathrm{s},(\bullet) ; \eta_{\text {in }}=10.8 \mathrm{mPa} \cdot \mathrm{s},(\bullet)$; $\eta_{\text {in }}=22.5 \mathrm{mPa} \cdot \mathrm{s},(\bullet)$.

Next, we varied the droplet size for a set of different surface tensions, under fixed flow velocity. We then rescaled the maximum deformation by $C a^{2 / 3}$ and obtained a collapse of the data on a master curve (figure 7.6(b)). The power law is a good approximation of the master curve:

$$
\delta_{\max }=0.8 C a^{2 / 3} R^{* 3.7}
$$

The resulting deformation is therefore a function of both Capillary number and a geometric dimensionless parameter. For Taylor sheared droplets, the power law exponent in $C a$ is 1 . However when a droplet moves in a elongational flow field, the flow profile also contributes to the deformation [110, 111, 116] a feature not captured in the Taylor sheared droplet experiment. In addition, effects arising from the confinement of the droplets in the channel might also contribute to the dissipation. It is interesting to note that although the exponent of the 

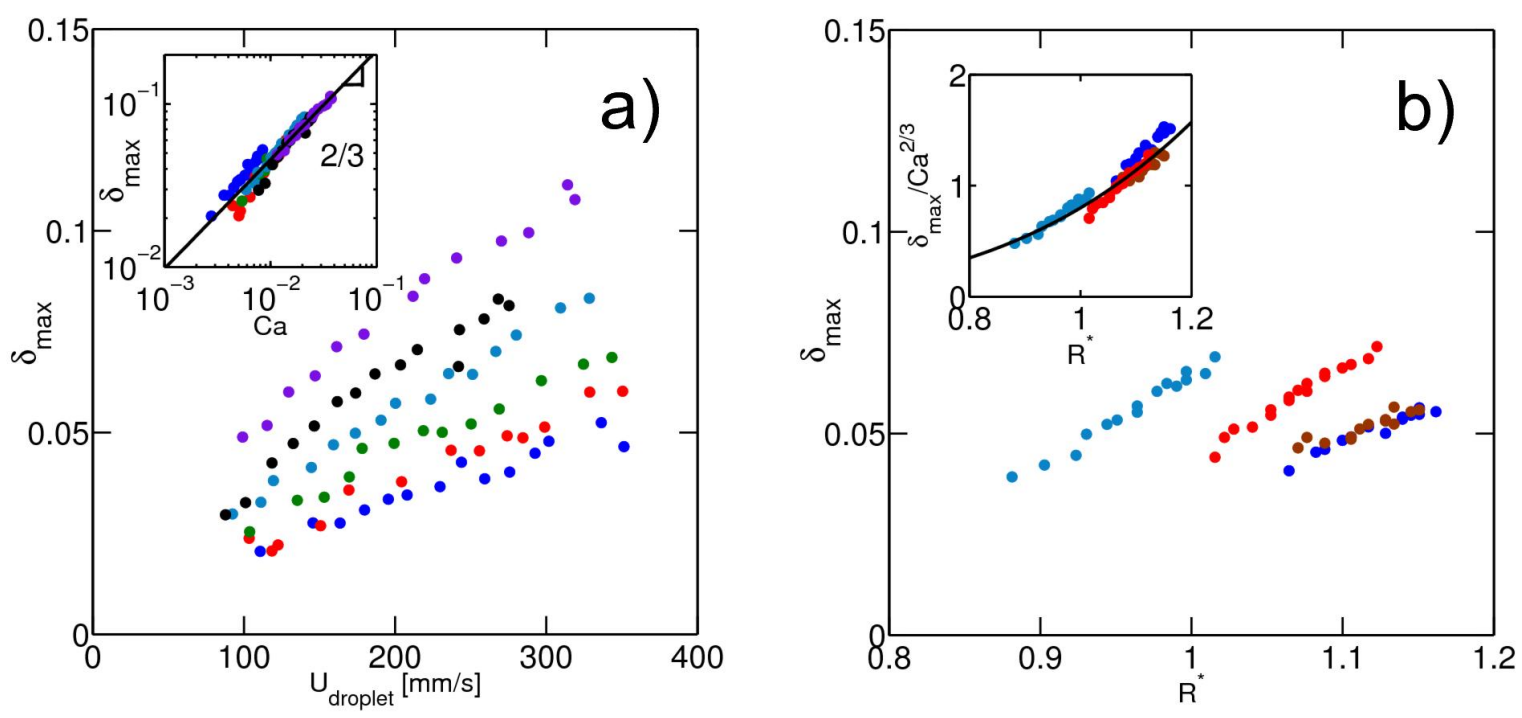

Figure 7.4 (a) Dependence of the droplet maximum deformation $\left(\delta_{\max }\right)$ on the Capillary number $(\mathrm{Ca})$ in conditions of fixed droplet radius $R=54 \mu \mathrm{m} \pm 8 \%$. Inset: Collapse of the droplet maximum deformation on a master curve $\delta_{\max } \propto \mathrm{Ca}^{2 / 3}$ (black line). (b) Dependence of the droplet maximum deformation on the size of the droplet $\left(R^{*}\right)$ for droplet speed $U_{\text {droplet }}=$ $330 \mathrm{~mm} \cdot \mathrm{s}^{-1} \pm 15 \%$. Inset: Collapse of the deformation data on a curve of equation $\delta_{\max }=$ $0.8 \mathrm{Ca}^{2 / 3} R^{* 3.7}$. Different colors are attributed to different surface tensions, according to the color code: $\gamma=49.5 \mathrm{mN} \cdot \mathrm{m}^{-1},(\bullet) ; \gamma=43.5 \mathrm{mN} \cdot \mathrm{m}^{-1},(\bullet) ; \gamma=29.2 \mathrm{mN} \cdot \mathrm{m}^{-1},(\bullet) ; \gamma=$ $23.9 \mathrm{mN} \cdot \mathrm{m}^{-1},(\bullet) ; \gamma=19.5 \mathrm{mN} \cdot \mathrm{m}^{-1},(\bullet) ; \gamma=14.5 \mathrm{mN} \cdot \mathrm{m}^{-1},(\bullet) ; \gamma=10.6 \mathrm{mN} \cdot \mathrm{m}^{-1}$, $(\bullet)$.

Capillary number can probably be derived from theoretical argument, there is no specific reason to obtain a power law relationship for the size dependence. The droplet size probably has a strong influence on the flow around the droplet due to the confinement, explaining the strong dependence of the droplet deformation on its size. Further hydrodynamic analysis including the effect of the confinement on droplet deformation is of interest and will be detailed in the next chapter.

In the following section, we will use the data relating deformation and surface tension (Eq. 7.1) as a phenomenological calibration of the system. The interfacial tension is expressed as a function of the droplet parameters $\delta_{\max }, U_{\text {defo }}, R$ and a constant $k=0.8$, by the following expression:

$$
\gamma=\eta_{\text {out }} U_{\text {defo }}\left(\frac{k}{\delta_{\text {max }}}\right)^{1.5} R^{* 5.5}
$$


It can be determined from the expression that a size fluctuation of $2 \%$ in droplet size (as usually obtained in microfluidic flow focussing) leads to a measurement error of $\sim 10 \%$ which sets the maximum resolution for the measurement (figure 7.5). Using all our experimental data, we can determine the surface tension $\gamma_{\mathrm{c}}$ obtained from the model equation as a function of the fluid-fluid interfacial tension $\gamma_{\mathrm{m}}$ (figure 7.5), inset.

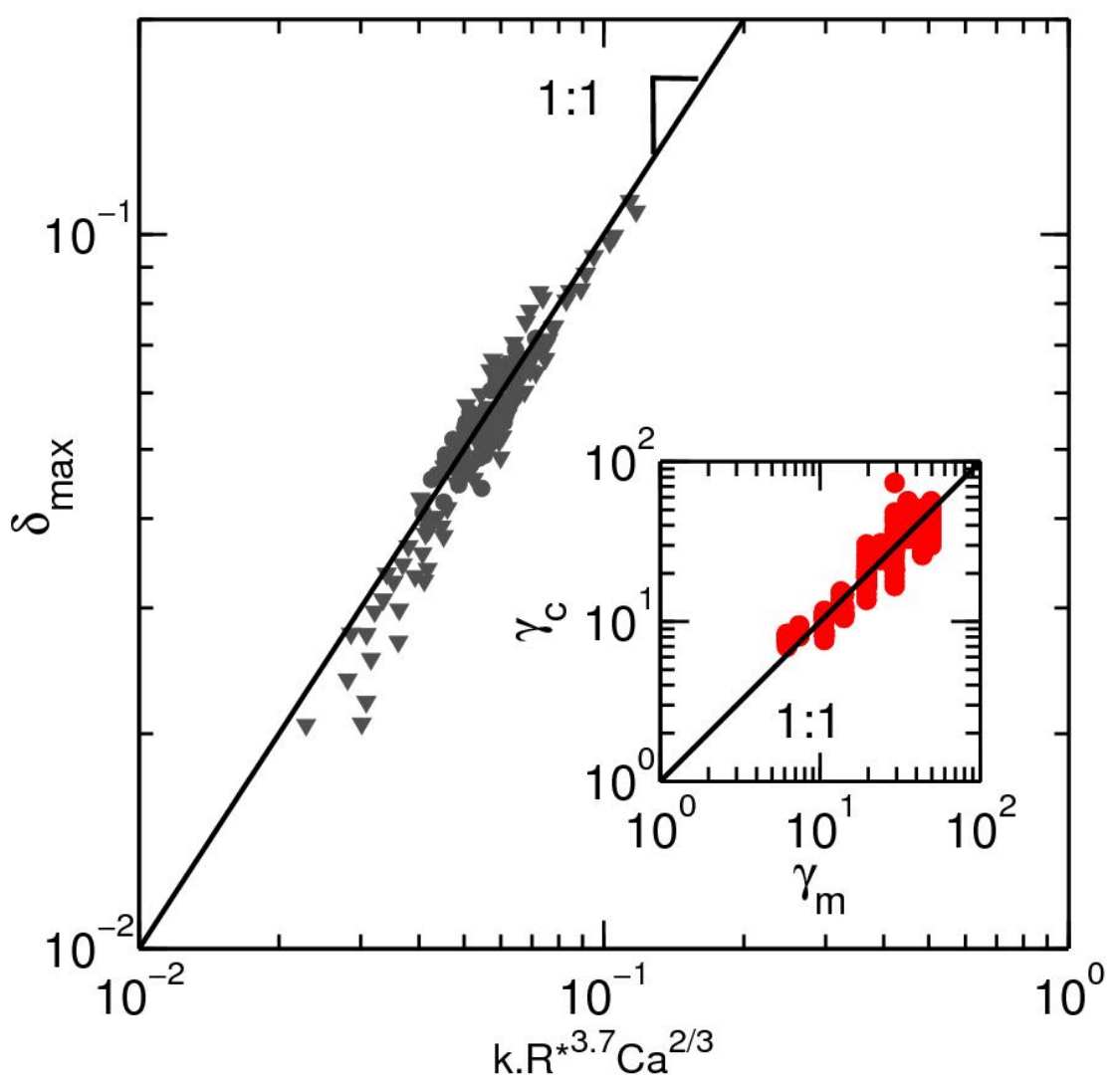

Figure 7.5 Collapse of all experimental values on a master curve using the empirical scaling equation for the surface tension (equation 7.2). Both experimental data are represented, where the speed of the droplet $(\bullet)$, and the size $(\Delta)$ are varied. The black line is a slope one as a guide for the eyes. The inset depicts the values of the surface tension for solution of ethanol in water and HFE-7500, as they are measured in pendant drop method, $\gamma_{\mathrm{m}}$, against the value calculated from the deformation profile in microfluidic channel $\gamma_{\mathrm{c}}$. The black line has a slope 1 corresponding to an ideal match between the 2 measurements. The dispersion of the data from this match due to the error made on the deformation is visible here. 


\subsection{Dynamics of surfactant adsorption: Microfluidics}

\subsubsection{Droplet deformation in presence of surfactants}

The surfactant adsorption is then measured in our microfluidic chip. Fixing the flow conditions to $Q_{\text {tot }}=72 \mu \mathrm{L} \cdot \mathrm{min}^{-1}\left(Q_{H 2 O}=2 \mu \mathrm{L} \cdot \mathrm{min}^{-1}, Q_{\text {oil }}=50 \mu \mathrm{L} \cdot \min ^{-1}, Q_{\text {sep }}=20 \mu \mathrm{L} \cdot \mathrm{min}^{-1}\right)$, we measure the deformation of the droplet in a series of expansions distributed along a delay line.
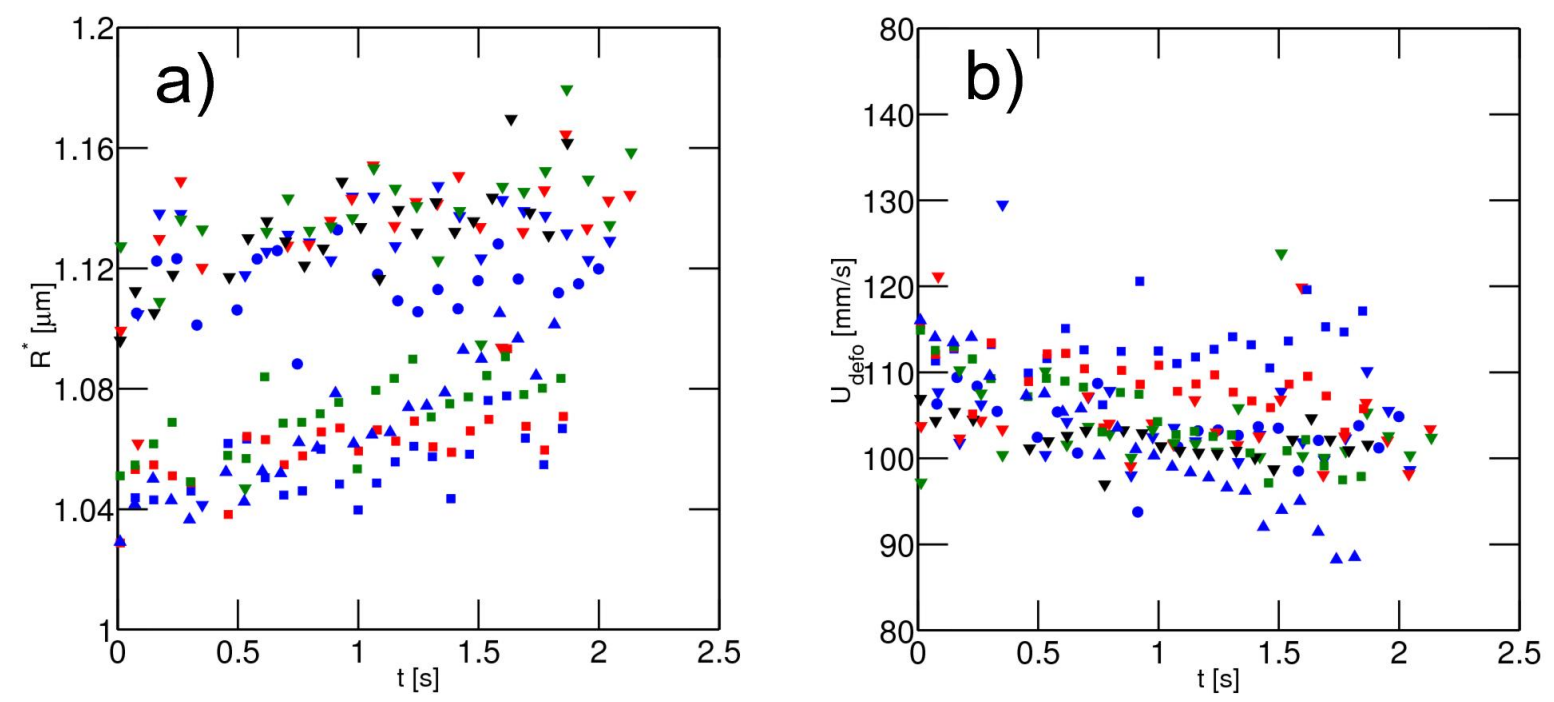

Figure 7.6 Size (a) and speed (b) of the droplet along a channel during the dynamic measurement of the surface tension, for $C_{\mathrm{PEG}-\mathrm{PFPE}}=1.3 \times 10^{-3} \mathrm{~mol} \cdot \mathrm{m}^{-3}(\bullet) ; C_{\mathrm{PEG}-\mathrm{PFPE}}=$ $1.3 \times 10^{-2} \mathrm{~mol} \cdot \mathrm{m}^{-3},(\mathbf{\nabla}) ; \quad C_{\text {PEG-PFPE }}=2.6 \times 10^{-2} \mathrm{~mol} \cdot \mathrm{m}^{-3},(\boldsymbol{\nabla}) ; C_{\mathrm{PEG}-\mathrm{PFPE}}=$ $5.2 \times 10^{-2} \mathrm{~mol} \cdot \mathrm{m}^{-3},(\mathbf{v}) ; C_{\mathrm{PEG}-\mathrm{PFPE}}=10.4 \times 10^{-2} \mathrm{~mol} \cdot \mathrm{m}^{-3},(\mathbf{v}) ; C_{\mathrm{PEG}-\mathrm{PFPE}}=$ $1.3 \times 10^{-1} \mathrm{~mol} \cdot \mathrm{m}^{-3},(\boldsymbol{\square}) ; C_{\mathrm{PEG}-\mathrm{PFPE}}=2.6 \times 10^{-1} \mathrm{~mol} \cdot \mathrm{m}^{-3},(\mathbf{\square}) ; C_{\mathrm{PEG}-\mathrm{PFPE}}=$ $5.2 \times 10^{-1} \mathrm{~mol} \cdot \mathrm{m}^{-3},(\mathbf{\square}) ; C_{\mathrm{PEG}-\mathrm{PFPE}}=1.3 \mathrm{~mol} \cdot \mathrm{m}^{-3},(\boldsymbol{\Lambda})$.

Along the whole channel, we observe a weak but measurable variation of the droplet size and speed as shown in figure 7.6. The apparent size of the droplet $R^{*}$ is varying at most by $4 \%$, velocities at most by $10 \%$. These small variations integrate microfabrication uncertainties on the channel depth and the change of boundary conditions at the interface[117], and the change in the thickness of the lubrication layer thickness around a confined droplet. In the case of large droplets, the lubrication layer thickness given by Bretherton[118] is of order $\sim \mathrm{HCa}^{2 / 3}$. The order of magnitude for this layer in our case is at most $6 \mu \mathrm{m}$. Therefore it is not excluded that the change of surface tension upon adsorption would modulate the layer thickness leading to a 
change of the apparent droplet area. We believe that these variations are linked to the effect of the surfactant adsorption, by modification of Bretheron film or interface rigidification, as they are more pronounced at higher surfactant concentrations. In the following we will account for these variations by adjusting for the velocity and droplet size measured in each succesive expantsion considered.

In the first expansion, the droplet remains circular with non measurable deformation while larger deformations are observed at the end of the microfluidic channel, indicating that the surface tension has decreased (Figure 7.7(a)). Using our calibration (equation 7.2) we derive the time evolution of the surface tension (Figure 7.7 (b)). Accounting for the apparent size and velocity of the droplet, we obtain plateau values of the surface tension for the highest surfactant concentrations, even in regions where the maximum deformation $\delta_{\max }$ still increases. As expected the values of the plateau depends on surfactant concentration and are consistent with the pendant drop measurements (Figure 7(c)).

We compute the interfacial age, by taking into account the different variations of the droplet speed discussed above. First, at the expansion, the mass conservation induce a general decrease of fluid speed in the x-direction by roughly a factor of three. For each measured velocity profile, we define two additional velocity, $U_{\max }$ and $U_{\min }$, respectively corresponding to the velocity of the droplet in the channel, and in the center of the deformation chamber as shown in figure 7.2. The velocities are extracted in the subset of expansion regularly displayed along the delay line. The age of interface is then computed iteratively from expansion $(\mathrm{N}-\mathrm{n})$ to expansion $(\mathrm{N})$, separated by a distance $L n$, where $\mathrm{n}$ is the number of expansions separating two measurement points, and $L$ the length of the chamber and of the channel. We use generally $n=5$. To refine our time precision, the speed in between two points of measurement is estimated by taking the average of $U_{\min }$ and $U_{\max }$, resulting in the following equation:

$$
t_{\mathrm{N}}=t_{\mathrm{N}-\mathrm{n}}+\frac{L n}{\frac{1}{2}\left[U_{\max }(N)+U_{\max }(N-n)\right]+\left[U_{\min }(N)+U_{\min }(N-1)\right]}
$$

This series starts with the initial condition $t_{1}$ which is the time to travel from the droplet production to the first expansion. A higher order polynomial is fitted through the time line in order to extract the equation linking position at any point of the channel to the age of the interface. 


\begin{tabular}{ccc}
\hline Parameter & Value & Units \\
\hline \hline$\kappa$ & $3 \times 10^{3}$ & $\mathrm{~m}^{3} \cdot \mathrm{mol}^{-1}$ \\
$\Gamma_{\infty}$ & $3.4 \times 10^{-6}$ & $\mathrm{~mol} \cdot \mathrm{m}^{-2}$ \\
$k_{\mathrm{des}}$ & $6 \times 10^{-3}$ & $\mathrm{~s}^{-1}$ \\
$k_{\mathrm{ads}}=k_{\mathrm{des}} \kappa$ & 18 & $\mathrm{~m}^{3} \cdot \mathrm{mol}^{-1} \cdot \mathrm{s}^{-1}$ \\
$\beta^{*}=k_{\mathrm{ads}} \Gamma_{\infty}$ & $6 \times 10^{-3}$ & $\mathrm{~cm} \cdot \mathrm{s}^{-1}$ \\
\hline
\end{tabular}

Table 7.1 PEG-PFPE surfactant parameters obtained from microfluidic tensiometry. These parameters have to be compared to the one extracted in pendant drop method, see table 6.1

\subsubsection{Dynamic microfluidic tensiometry}

At high surfactant concentrations, the interfacial tension reaches an equilibrium value in less than one second. At lower surfactant concentrations, the relaxation to equilibrium is slower and the equilibrium value larger, as expected. We observe an increase in the experimental error at high surface tension because deformation of order $1 \%$ is at the limit of our optical resolution. The accuracy of the measurement is maximal at low surface tension $\left(\sim 1 \mathrm{mN} \cdot \mathrm{m}^{-1}\right)$. This makes the system interesting for highly efficient surfactant, possibly used for microemulsion production. This accuracy at low surface tension constitutes a significant improvement to the pendant drop method where low surface tension $\gamma<5 \mathrm{mN} \cdot \mathrm{m}^{-1}$, is at the limit of the method, see figure 7.8. In addition, the deformation experiments can be performed even for $\Delta \rho=0$, for which pendant drop tensiometry would fail.

As an example, the interface PBS (Phosphate Buffer Saline) 1X and HFE-7500 with a concentration of Krytox-H, a charged perfluorosurfactant, around $C=0.65 \mathrm{~mol} \cdot \mathrm{m}^{-3}$, is measured to have an equilibrium surface tension. $\gamma_{\mathrm{eq}}<5 \mathrm{mN} \cdot \mathrm{m}^{-1}$ with the pendant drop method, but could not be determined precisely. In microfluidics the deformation for such system is large. $\delta_{\max }=0.32$ for values of $R^{*}=1$ and $U_{\text {defo }}=70 \mathrm{~mm} \cdot \mathrm{s}^{-1}$. This leads to a surface tension of $\gamma_{\mathrm{eq}}=0.5 \mathrm{mN} \cdot \mathrm{m}^{-1}$ based on our calibration.

We are now interested to determine the mode of surfactant adsorption to the interface from the dynamic surface tension measured on chip. We first analyzed the asymptotic behaviors of the dynamics. Contrary to the pendant drop experiments, a fit with a power law in $t^{-1 / 2}$ was not possible. However, the data could be fitted by an exponential relaxation, observed for example in transfer limited kinetics. We extracted from the fit the value of $\gamma_{e q}$ and of the 
characteristic time $\tau$ as a function of surfactant concentration according to (equation 7.4):

$$
\gamma-\gamma_{e q} \sim \exp (-t / \tau)
$$

The equilibrium values obtained as a function of surfactant concentration are in full agreement with the pendant drop measurements showing the reliability of the method, see figure 7.7 (c). In the transfer limited regime, the adsorption - desorption process is treated as a binding reaction involving a fixed number of binding sites at the interface. The definition of the adsorption and desorption rate constant $k_{\text {ads }}$ and $k_{\text {des }}$, as well as the surface coverage as a function of time is obtained with:

$$
\frac{\partial}{\partial t}\left(\frac{\Gamma(t)}{\Gamma_{\infty}}\right)=k_{\mathrm{ads}} C\left(1-\frac{\Gamma}{\Gamma_{\infty}}\right)-k_{\mathrm{des}} \frac{\Gamma}{\Gamma_{\infty}}
$$

leading to

$$
\frac{\Gamma(t)}{\Gamma_{\infty}}=\frac{\Gamma_{\mathrm{eq}}}{\Gamma_{\infty}}(1-\exp (-t / \tau))
$$

where

$$
\tau=\frac{1}{k_{\mathrm{des}}} \frac{1}{1+\kappa C}
$$

The Langmuir isotherm $\Gamma_{\text {eq }}(C)$ is recovered with the Langmuir equilibrium constant expressed as $\kappa=k_{\text {ads }} / k_{\text {des }}$. The expression of $\tau$ is similar to the one given in Li et al. [25]. Following the assumption that the surface tension changes are proportional to the changes in the droplet surface coverage, we obtain the expression for the relaxation time scale as a function of desorption rate and the Langmuir parameter $\kappa$. Unfortunately, the accuracy of our experimental data is not satisfactory enough in the region of the CMC and therefore does not provide a convenient range of concentration to test this scaling. If the micelles contribute to the kinetics, assuming that neither the dissociation nor the diffusion are the limiting step, then the expression of $\tau$ is correct even above the CMC. In contrast if the dissociation is infinitely slow, the time scale is expected to level to a constant value above the CMC. The experimental data lie within these two asymptotic limits:

$$
\frac{1}{k_{\mathrm{des}}} \frac{1}{1+\kappa C}<\tau<\frac{1}{k_{\mathrm{des}}} \frac{1}{1+\kappa C}+\frac{1}{k_{\mathrm{des}}} \frac{1}{1+\kappa C_{\mathrm{CMC}}}
$$

A value of $k_{\mathrm{des}}=6 \times 10^{-3} \mathrm{~s}^{-1}$ can be extracted from the behavior of $\tau$ (Figure 7.7 (d)). We use then the knowledge of $\kappa$ to extract the adsorption rate $k_{\mathrm{ads}}=k_{\mathrm{des}} \kappa=18 \mathrm{~m}^{3} \cdot \mathrm{mol}^{-1} \cdot \mathrm{s}^{-1}$. The rate constants can also be expressed in the form [98] $\beta^{*}=k_{\mathrm{ads}} \Gamma_{\infty}=6 \times 10^{-3} \mathrm{~cm} \cdot \mathrm{s}^{-1}$. For 
clarity, all the data are provided in table 7.1 .

As a further evidence that the PEG-PFPE micelles can directly adsorb to the interface we note that a jumps occurs in the droplet size while crossing the $\mathrm{CMC}$, see figure 7.6, in condition of constant flow rate. This is an indication that the surfactant adsorption time is small enough to have an effect already at the droplet production. The frequency gives us a typical interface age of the order of $t_{\text {prod }}=16 \mathrm{~ms}$ before detachment.

We prove that this method is suitable for a quantitative analysis of the surfactant kinetic properties. Although the accuracy of the measurement value of $k_{\mathrm{des}}$ is limited by the large error bars due to the determination of the time-scale and the crude approximations of the model, we provide here the first estimate of the rate constants for the PEG-PFPE surfactant using our microfluidic chip. 

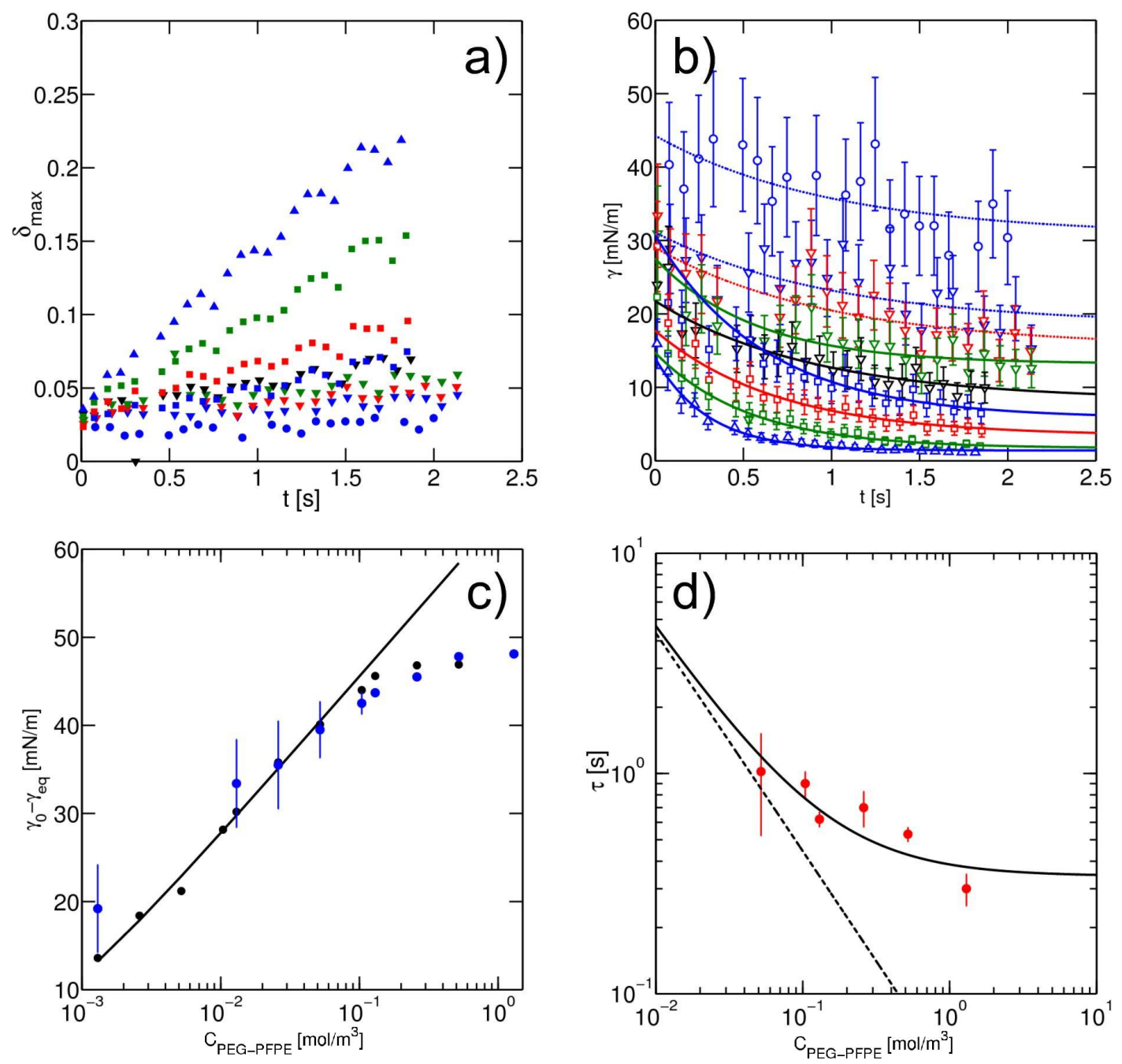

Figure 7.7 (a) Maximum deformation of the droplet as a function of the time as recorded on-chip. Here the measured points correspond to a subset where one chamber out of five is analyzed. The concentrations of surfactant used are: $C={ }_{\text {PEG-PFPE }} 1.3 \times 10^{-3} \mathrm{~mol} \cdot \mathrm{m}^{-3}$ $(\bullet) ; C_{\text {PEG-PFPE }}=1.3 \times 10^{-2} \mathrm{~mol} \cdot \mathrm{m}^{-3}, \quad(\boldsymbol{\nabla}) ; C_{\mathrm{PEG}-\mathrm{PFPE}}=2.6 \times 10^{-2} \mathrm{~mol} \cdot \mathrm{m}^{-3}$, $(\boldsymbol{\nabla}) ; C_{\text {PEG-PFPE }}=5.2 \times 10^{-2} \mathrm{~mol} \cdot \mathrm{m}^{-3},(\boldsymbol{\nabla}) ; C_{\text {PEG-PFPE }}=10.4 \times 10^{-2} \mathrm{~mol} \cdot \mathrm{m}^{-3}$, ( $\mathbf{v}) ; C_{\mathrm{PEG}-\mathrm{PFPE}}=1.3 \times 10^{-1} \mathrm{~mol} \cdot \mathrm{m}^{-3},(\boldsymbol{\square}) ; C_{\mathrm{PEG}-\mathrm{PFPE}}=2.6 \times 10^{-1} \mathrm{~mol} \cdot \mathrm{m}^{-3},(\mathbf{\square})$; $C_{\mathrm{PEG}-\mathrm{PFPE}}=5.2 \times 10^{-1} \mathrm{~mol} \cdot \mathrm{m}^{-3},(\square) ; C_{\mathrm{PEG}-\mathrm{PFPE}}=1.3 \mathrm{~mol} \cdot \mathrm{m}^{-3},(\boldsymbol{\Delta})$. (b) Surface tension derived from the maximum droplet deformation with the empirical scaling, (equation 7.2). The open symbols correspond to concentration in (a). The solid lines are the best fits found for each concentration, using an exponential fitting of the late kinetics, based on (equation 6.3). For the dotted line the fit has been done with values of $\tau$ fixed to one in order to extract the equilibrium surface tension. (c) Comparison between the equilibrium values of the surface tension as a function of the surfactant concentration from pendant drop method $(\bullet)$, and from microfluidic experiments $(\bullet)$. The solid line is the fitting of the Langmuir-von Szyszkowsky equation, (equation 6.6). Dashed line represents the CMC. (d) Characteristic time scales $\tau$ extracted from late on-chip kinetics, (equation 7.6). Both lines represent the asymptotic expressions of $\tau$ in transfer limited, where micellar adsorption is allowed (solid line), and not allowed (dashed line), (equation 7.7), for a value of $k_{\mathrm{des}}=6.5 \times 10^{-3} \mathrm{~s}^{-1}$. 


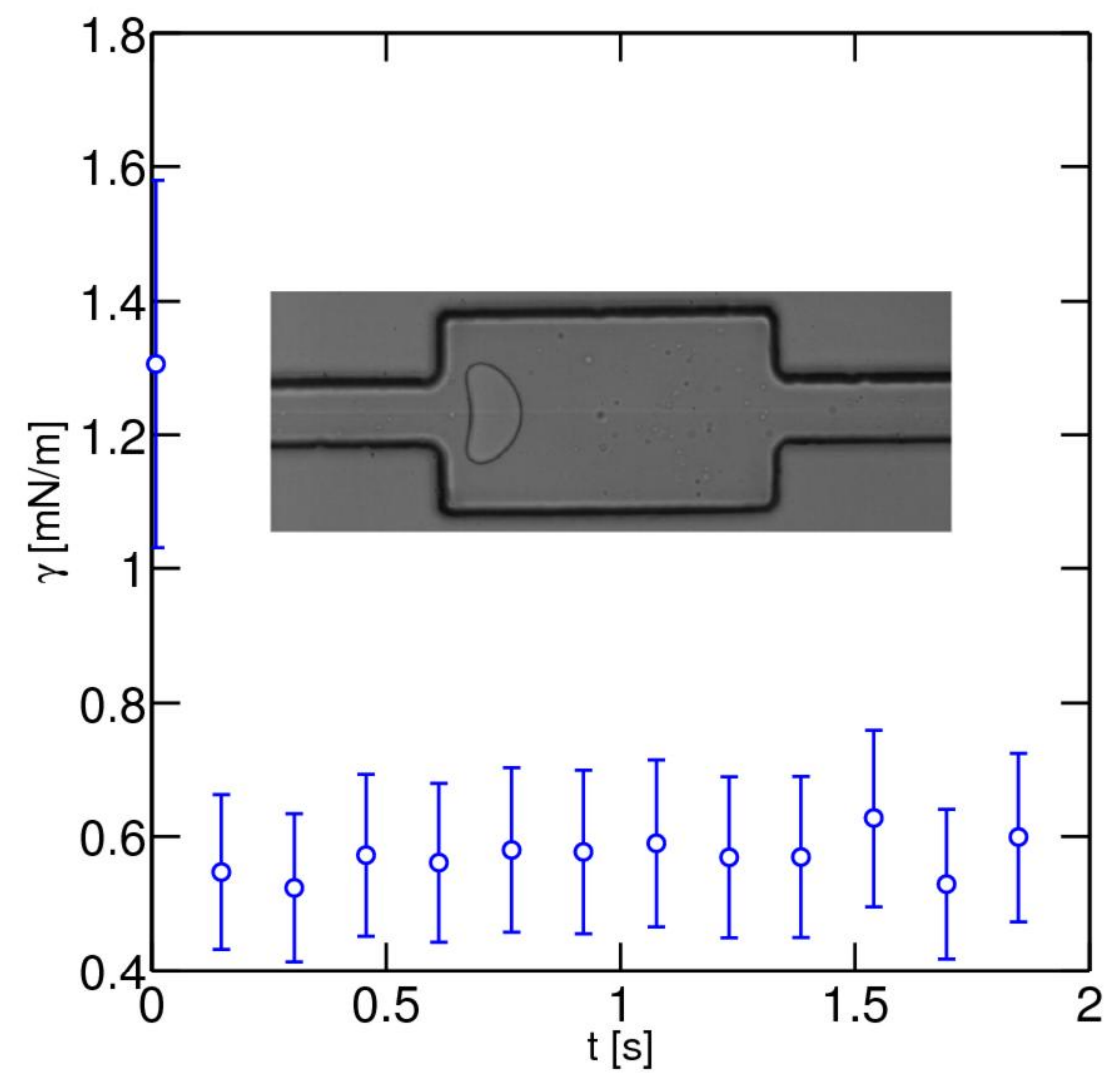

Figure 7.8 On chip dynamic tensiometry applied to the system Krytox-H $C_{\mathrm{K}-\mathrm{H}}=0.65 \mathrm{~mol} \cdot \mathrm{m}^{-3}$ in HFE-7500 and PBS 1X. The surface tensions measured are out of the range of the pendant drop method. Inset Micrograph of a droplet at its maximum deformation. 


\subsection{Discussion}

We have designed a microfluidic system to measure the surfactant adsorption in the conditions relevant to emulsification processes and provide a measurement of the rates of adsorption and desorption. The adsorption on chip is shown to be transfer-limited as one might expect in the presence of convective currents and at small dimensions. Quantitatively, the values we obtain for the adsorption rates provide estimates of the concentration cutoff above which the adsorption is changing from diffusion limited to transfer limited [98]. This concentration is expressed as $C_{\mathrm{d}}^{\max }=0.01 \beta^{*} \Gamma_{\infty} / D=4 \times 10^{-3} \mathrm{~mol} \cdot \mathrm{m}^{-3}$ in our case. A mixed-kinetic regime can be expected in the pendant drop experiments even in the absence of convection below the CMC. We hypothesize that the occurrence of the Plateau in the pendant drop measurement between the two asymptotic regimes relates to this mixed-kinetic behavior which suggests that a mixed-kinetic model has to be used to quantitatively analyses pendant drop experiments. In microfluidics, all experiments have been performed with concentrations above $C_{\mathrm{d}}^{\max }$ which confirms the influence of the transfer kinetics on the surfactant adsorption to the interface.

The cutoff length $R_{\mathrm{DK}}=D /\left(\Gamma_{\infty} k_{\mathrm{ads}}\right)$ [97] below which the adsorption processes are transfer limited is also estimated to be $R_{\mathrm{DK}} \sim 10 \mu \mathrm{m}$. In the pendant drop experiments, $R \gg R_{\mathrm{DK}}$ which is consistent with the diffusion limited regime observed at very small concentrations. In microfluidics, the adsorption is transfer limited even for droplet sizes around $100 \mu \mathrm{m}$ as a result of the convective currents present in the system. Indeed the Péclet Number $P e=U_{\text {droplet }} L / D$ is of order $10^{5}$ using the typical droplet velocity $U_{\text {droplet }} \sim 0.2 \mathrm{~m} / \mathrm{s}$ and the droplet size as typical $L$. Another cutoff length can be defined to account for the convective currents by replacing the diffusive effects by convective effects. Comparing the typical speed of adsorption $k_{\text {ads }} \Gamma_{\infty}$ describing the adsorption of molecules with the typical speed of the droplet relative to the oil (related to the amount of surfactant molecules available to the interface), we obtain a dimensionless number $k_{\mathrm{ads}} \Gamma_{\infty} / U \sim 3.10^{-4}$ indicating that the reaction kinetics is by far limiting the adsorption reaction in the presence of convection. Hence we do not expect significant change in the kinetics when the flow rates are varied in the experiments.

In general, the values of $\kappa, \Gamma_{\mathrm{eq}}$ and the rate constants $k_{\mathrm{ads}}$ and $k_{\mathrm{des}}$ are all consistent with values obtained for other systems. For example, $\Gamma_{\infty}$ is within the same order of magnitude compared to surfactant of the series $C_{\mathrm{n}} E_{\mathrm{m}}$ which have similar head group compared to our molecule [86, 97]. The long chain length of the perfluorosurfactant is therefore expected to be 
mainly oriented perpendicular to the interface without significantly folding onto the interface. A similar statement can be made based on the value of $k_{\text {ads }}$ which is only about three times larger than the one of $C_{14} E_{8}$ [86, 97]. Our method provides a measurement of the surface tension of a liquid-liquid interface during flow. We show here that we reach a transfer limited regime and this regime will dominate for droplet speeds even three order of magnitude slower (i.e. around $200 \mu \mathrm{m} \cdot \mathrm{s}^{-1}$ ) for $100 \mu \mathrm{m}$ droplets and for all speeds when the droplets are smaller than $10 \mu \mathrm{m}$.

Our results can be compared to the previous analysis of coalescence in microfluidic channels [74]. The coverage required to stabilize the emulsion (estimated to $\sim 10 \%$ [74] with a related surfactant) is reached in the early time kinetics $(\sim 10 \mathrm{~ms})$ where our current method is the least sensitive. Therefore making a direct link between dynamic tensiometry and the dynamics of emulsion stabilisation is not straightforward. In addition, emulsions are stabilised by Marangoni effects [119]. The good agreement between the equilibrium values in pendant drop tensiometry and the microfluidic experiments shows that Marangoni stresses do not significantely influence the interfacial measurement in our range of parameters [120]. However expanding the analysis to the data generated during the expansion and relaxation of the droplet could probably be used to measure interfacial rheology properties relevant to understand coalescence [90, 104, 119].

\subsection{Conclusions}

In summary, we developed a method to measure surfactant adsorption in microfluidics through the deformability of droplets in shear flow. We exemplify its use on the analysis of the adsorption of a perfluorinated molecule of practical interest for microfluidic applications. Standard droplet tensiometry experiments provide estimates of the surfactant properties in a diffusionlimited case while the microfluidic platform show that the kinetics of adsorption is transfer limited. The microfluidic experiments enable us to estimate the adsorption and desorption constant and provide equilibrium values compatible with the pendant drop experiments. Our microfluidic system can further be used to analyses the behavior of other surfactant molecules, for example ionic molecules or more generally to analyzes the transient states of active and reactive interfaces.

The study of the droplet deformation in microchannels also elucidated that a contribution 
of the droplet confinement $(2 \mathrm{R} / \mathrm{W})$ should be taken into account for the description of the droplet maximum deformation $\delta_{\max }$ in the microchannels. Such contribution is not present in the standard two dimensional and three dimensional model used to date. In the following chapter we will present the study of the droplet deformation and relaxation in our geometry in order to better quantify the influence of the confinement on the droplet deformation. 


\section{8}

\section{Deformation of droplet interface in microfluidics environments}

\subsection{Introduction}

In the previous chapter we have presented a microfluidic method able to measure the dynamic surface tension of a droplet in the presence of a surfactant. We established an empirical dependence of the droplet deformation, on the droplet size and the Capillary number. In this chapter we present the study of the droplet deformation and relaxation in a planar sudden expansion. The aim of this chapter is to provide better understanding of the influence of the microfluidic confinement on the droplet deformation. We will compare the experimental droplet deformation to existing two dimensional and three dimensional models. In addition, we compare the behavior of fresh interfaces and surfactant-laden interfaces, in order to measure the possible influence of the Marangoni effects during the interface deformation and relaxation.

In the first part, we will describe the deformation of the droplet entering the planar expansion. We show how the droplet maximum deformation $\delta_{\max }$ is influenced by the droplet confinement, and define two different regimes of deformation. In this study we will also compare our experimental results with a numerical two dimensional model based on the Darcy equation. The differences between the experiment and this model will be discussed. 
The second part of this chapter will be dedicated to the droplet deformation when reentering the channel. This part of the deformation profile can be described by the general model of a spheroid deformation in an elongational flow profile derived from work of Taylor[110]. We used this description to estimate the deviations of the deformation in a microfluidic channel compared to the three dimensional model.

The third part of this chapter will be focused on the relaxation of the droplet from the maximum deformation to its rest state, which takes place in the chamber following the expansion. We will discuss the appearance of an inertial regime at high droplet speed. This regime is characterized by periodic oscillations of the droplet. We will apply a model of a damped harmonic oscillator to fit these oscillations and show that the frequency can be described by the droplet surface tension and volume. In presence of surfactant we will present a further way to measure dynamic surface tension based on the measurement of the oscillation frequency of the droplets. We acknowledge Dr. Erfan Khadivar for the two dimensional numerical simulations that have been performed in the present chapter.

\subsection{Droplet deformation in a planar sudden expansion}

The flow of soft objects in microfluidics is of great importance in the study of biological material such as red blood cells [121-123], capsules and vesicles, as well as emulsion and foams in confined environments. In microfluidics the study of droplet flow can be considered as a simplified model to describe the behavior of complex interfaces. The omnipresence of walls in confined environments, imposes strong constraints on the flow profile. For example small channel dimensions amplify the importance of boundary effects relative to the bulk effects, and cancel the inertial forces (at low Reynolds numbers). Furthermore the size of the droplet will influence the mode of transport in the channel. Advantageously, microfluidics techniques provide a high throughput generation of controlled droplet sizes, that enable the production of monodisperse emulsions [43, 124], and allow an easy fine tuning of the droplet confinement. To date a large number of works has investigated droplet flow in microchannels [41, 115, 125], as well as phenomena like clogging[126] and coalescence[51, 127].

On its own the deformation of a droplet in a flow field constitutes an interesting question in soft matter. The analysis of the interface deformation under shear stress gives information on the rheological and mechanical properties of the interface[128, 129], particularly in the presence of an adsorbed layer. Direct applications are the methods employed to determine 
the surface tension by hydrodynamic deformation of the interface[13]. A further motivation is the prediction of the stability of foams and emulsions, via the analysis of droplet break up conditions [116, 130]. From the pioneering work of Taylor[110, 131], the deformation of droplets has been studied experimentally and numerically with various flow profiles, viscosity ratios and Capillary numbers[132]. The expression of the deformation is particularly well described in the case of small deformations of a spherical shape.

\subsubsection{Experimental method}

The experimental method is analogous to the one used for the determination of the surface tension in the previous chapter. The droplet deformation is provoked by a sudden divergence of the flow at the opening of a channel on a 3:1 expansion. It is calculated by the ratio $\delta=$ $(L-l) /(L+l)$, where $L$ is the axis of the droplet in x-direction and $l$ its axis in y-direction. The droplet confinement is estimated by the ratio of the droplet radius $R$ to the channel height $H$ and width $W$, see figure 8.1 . The profile of deformation is recorded at high speed and the maximum deformation $\delta_{\max }$ is extracted from the profile, see figure 8.2 . In this work $W$ is kept constant, while $R$ and $H$ are varied in a controlled way. We modify the droplet size $R$ by changing the relative oil / water flow rate at a flow focusing junction. The range of droplet sizes accessible at the production is directly limited by the channel biggest dimension at the production [124]. We change the channel height $H$ using standard soft-lithography methods in order to produce NOA microfluidics chips[64] with three different height.

We define the Capillary number as: $C a=\eta_{\mathrm{c}} U_{\text {defo }} / \gamma$, where $\eta_{\mathrm{c}}$ is the viscosity of the continuous phase, $U_{\text {defo }}$ is the droplet speed at the maximum deformation, and $\gamma$ the surface tension. The dependency of the droplet maximum deformation upon $C a$, is obtained by changing the surface tension, and the droplet speed. We vary the surface tension by using different water ethanol mixtures as aqueous phase[133], $\gamma=49.5-10.6 \mathrm{mN} \cdot \mathrm{m}^{-1}$ for ethanol proportions between $C_{\text {eth }}=0-40 \%(\mathrm{w} / \mathrm{w})$. The speed of the droplet can also be varied with little effects of the droplet size by tuning the flow rate of a supplementary oil stream, situated after the production.

The oil phase consists in pure Novec-7500 fluorinated oil (3M) used as received. 


\begin{tabular}{lll}
\hline$C_{\text {eth wt. } \%}$ & $\gamma \mathrm{mN} \cdot \mathrm{m}^{-1}$ & $\rho_{\text {in }} \mathrm{kg} \cdot \mathrm{m}^{-3}$ at $20^{\circ} \mathrm{C}$ \\
\hline \hline 0 & 49.6 & 998.23 \\
2 & 43.5 & 994.53 \\
5 & 36.7 & 989.38 \\
10 & 29.2 & 981.87 \\
15 & 23.9 & 975.14 \\
20 & 19.5 & 968.64 \\
30 & 14.1 & 953.82 \\
40 & 10.6 & 935.18 \\
\hline
\end{tabular}

Table 8.1 Equilibrium surface tensions of water/ethanol mixture and HFE-7500, and their corresponding densities.

\subsubsection{Numerical two-dimensional model}

Numerically we consider two-dimensional droplets in the Hele-Shaw limit which is confined between the top and bottom walls of the microchannel. The height of the channel, $H$, is assumed to be much smaller than the radius $R$ of a flat circular droplet and the width of the channel $W$. Both the dispersed phase (d) and the continuous phase (c) are incompressible liquids with a dynamic viscosity $\eta_{\mathrm{i}}, \in\{\mathrm{c}, \mathrm{d}\}$. In the Hele-Shaw limit, the flow velocities locally follow a Poiseuille flow profile. Deviations from the Poiseuille profile will become apparent at distance to the walls of the order of the cell height $H$ or smaller. The depth averaged velocity in the droplet and the continuous phase in-plane coordinates $x$ and $y$ is governed by the two-dimensional Darcy equation

$$
\mathbf{U}_{\mathrm{i}}=-\alpha_{\mathrm{i}} \nabla P_{\mathrm{i}} \quad \text { on } \quad \Omega_{\mathrm{i}} \quad \text { with } \quad \mathrm{i} \in\{\mathrm{d}, \mathrm{c}\},
$$

where $\alpha_{\mathrm{i}}=H^{2} / 12 \eta_{\mathrm{i}}$ is the mobility, $P_{\mathrm{i}}(x, y)$ the pressure, and $\Omega_{\mathrm{i}} \subset \mathbb{R}^{2}$ the two dimensional domain occupied by phases (c) and (d). Employing the continuity equation $\boldsymbol{\nabla} \cdot \mathbf{U}=0$, the pressure in the dispersed and the continuous phase satisfies the Laplace equation, for the boundary conditions: non slip condition and no flux through the channel walls, constant velocity of the continuous phase through the inlet and outlet and continuity of the velocity profile through the droplet interface[134, 135].

$$
\nabla^{2} P_{\mathrm{i}}=0 \quad i \in\{d, c\}
$$


These conditions are used together with the Darcy equation (equation 8.1) to describe the velocity fields in both phases. We obtained the relevant boundary data of the velocity field solving the self-consistent integral equation which links the pressure field $P_{\mathrm{c}}$ of the continuous phase on the droplet contour $\Gamma_{\mathrm{cd}}$, the walls $\Gamma_{\mathrm{cw}}$, and the inlet and outlet of the channel $\Gamma_{0}$ to its normal derivative $\nabla_{\mathbf{n}} P_{\mathrm{c}}$. The pressure field of the continuous phase has to satisfy an integral equation of the explicit form:

$$
\begin{array}{r}
P_{\mathrm{c}}\left(\mathbf{r}_{0}\right)=\int_{\Gamma_{\mathrm{cw}}, \Gamma_{0}} P_{\mathrm{c}} \partial_{\mathbf{n}} \mathcal{G}\left(\mathbf{r}, \mathbf{r}_{0}\right) \mathrm{d} \ell-\int_{\Gamma_{0}} \mathcal{G}\left(\mathbf{r}, \mathbf{r}_{0}\right) \partial_{\mathbf{n}} P_{\mathrm{c}} \mathrm{d} \ell \\
-\int_{\Gamma_{\mathrm{cd}}}\left\{\alpha \gamma K \partial_{\mathbf{n}} \mathcal{G}\left(\mathbf{r}, \mathbf{r}_{0}\right)-(1-\alpha) P_{\mathrm{c}} \partial_{\mathbf{n}} \mathcal{G}\left(\mathbf{r}, \mathbf{r}_{0}\right)\right\} \mathrm{d} \ell,
\end{array}
$$

where $\alpha \equiv \alpha_{\mathrm{d}} / \alpha_{\mathrm{c}}, \gamma$ is the interfacial tension, $K$ is the local curvature, and $\mathbf{r}_{0}$ is defined as the position of the boundary of the continuous phase. The function $\mathcal{G}\left(\mathbf{r}, \mathbf{r}_{\mathbf{0}}\right)=-\frac{1}{2 \pi} \ln \left|\mathbf{r}-\mathbf{r}_{\mathbf{0}}\right|$ represents the free Green function of the two-dimensional Laplace equation, where $\mathbf{r}=(x, y)$ and $\mathbf{r}_{0}=\left(x_{0}, y_{0}\right)$ are respectively the fields and singular points. In order to numerically solve the self-consistent integral equation we have to introduce non-dimensional physical quantities. To this end, we chose a suitable length scale $L_{0} \equiv W$, time scale $T_{0} \equiv L_{0}^{3} / \alpha_{\mathrm{c}} \gamma$, and pressure scale $P_{0} \equiv \gamma / L_{0}$, and express any physical quantity in terms of these three basic units [135]. We define a modified Capillary number with $\mathrm{Ca}^{*} \equiv Q T_{0} / L_{0}^{2}$. According to this definition the modified Capillary number is linked to the Capillary number defined above by a geometrical prefactor $(W / H)^{2} \mathrm{Ca}[136]$. For the evaluation of the integrals in equation 8.4, we discretized the interface according to boundary elements methods [134], where each element is represented by cubic splines with periodic boundary conditions. We follow the temporal evolution of the interface $\Gamma_{\mathrm{cw}}$ by remeshing at each time step. This remeshing is carried out by cubic interpolation[135].

\subsubsection{Results}

First, we are interested in the influence of the droplet confinement on its deformation, in a sudden expansion geometry. For all experiments the droplet radius is situated at a value around $R \sim 50 \mu \mathrm{m} \pm 30 \%$. Each experiment is performed in a different channel height, respectively $H=34 \mu \mathrm{m}, H=50 \mu \mathrm{m}$, and $H=113 \mu \mathrm{m}$, and is composed of different runs, obtained by changing the surface tension of the system. See figure 8.1 .

As expected the maximum deformation, for a fixed droplet size and channel height, varies 


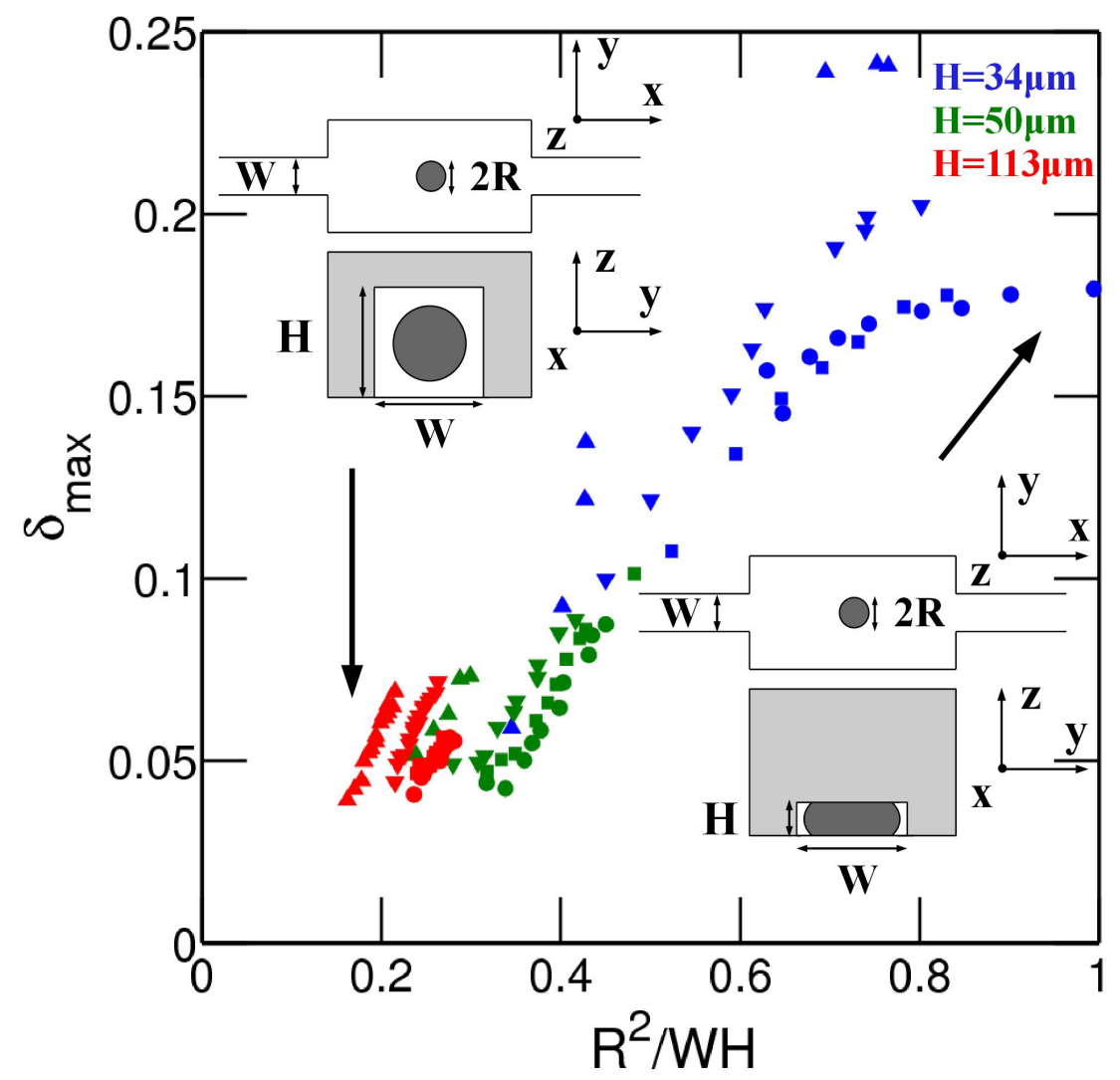

Figure 8.1 Dependency of the droplet maximum deformation on its size, in three channel depth: $H=34,50$, and $113 \mu \mathrm{m}$. Symbols are related to different surface tensions: $(\bullet) \gamma=$

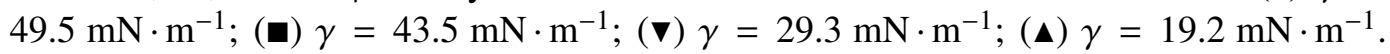
The insets are schematic views to appreciate the droplet morphology respective to the confinement in the microfluidic channel, and in the chamber. Both inset present a top view (top), and a cross-sectional view (bottom) of the channel. Two cases are displayed here. On the left, the case of a non-confined droplet with a diameter $2 R<W<H$. On the right, the case of a droplet confined in height with $H<2 R<W$.

with the inverse of the surface tension e.g. the Capillary number. More surprisingly, the channel height itself is found to affect the magnitude of the deformation, with an average value of the deformation for $H=34 \mu \mathrm{m}$ being on average three time higher than for $H=113 \mu \mathrm{m}$. In addition, for strongly confined droplets e.g. $H=34 \mu \mathrm{m}$, the values of the deformation reach a plateau around $R^{2} / W H=1$. The wingspan of the droplet at its maximum deformation, here $\delta_{\max } \sim 0.2$, is too small to reach the walls of the expansion. We can conclude that the droplet transversal deformation is not limited by the geometry of the expansion, and that the on-set of the plateau depends as expected on the geometrical quantities $R, W$ and $H$. 
We consider in the following part the experimental case of strongly confined droplets $H=$ $34 \mu \mathrm{m}$, and compare it to the numerical two dimensional simulation. Here, the three numeric control parameters: the droplet area, the total flux through the channel and the surface tension of the interface, are varied.

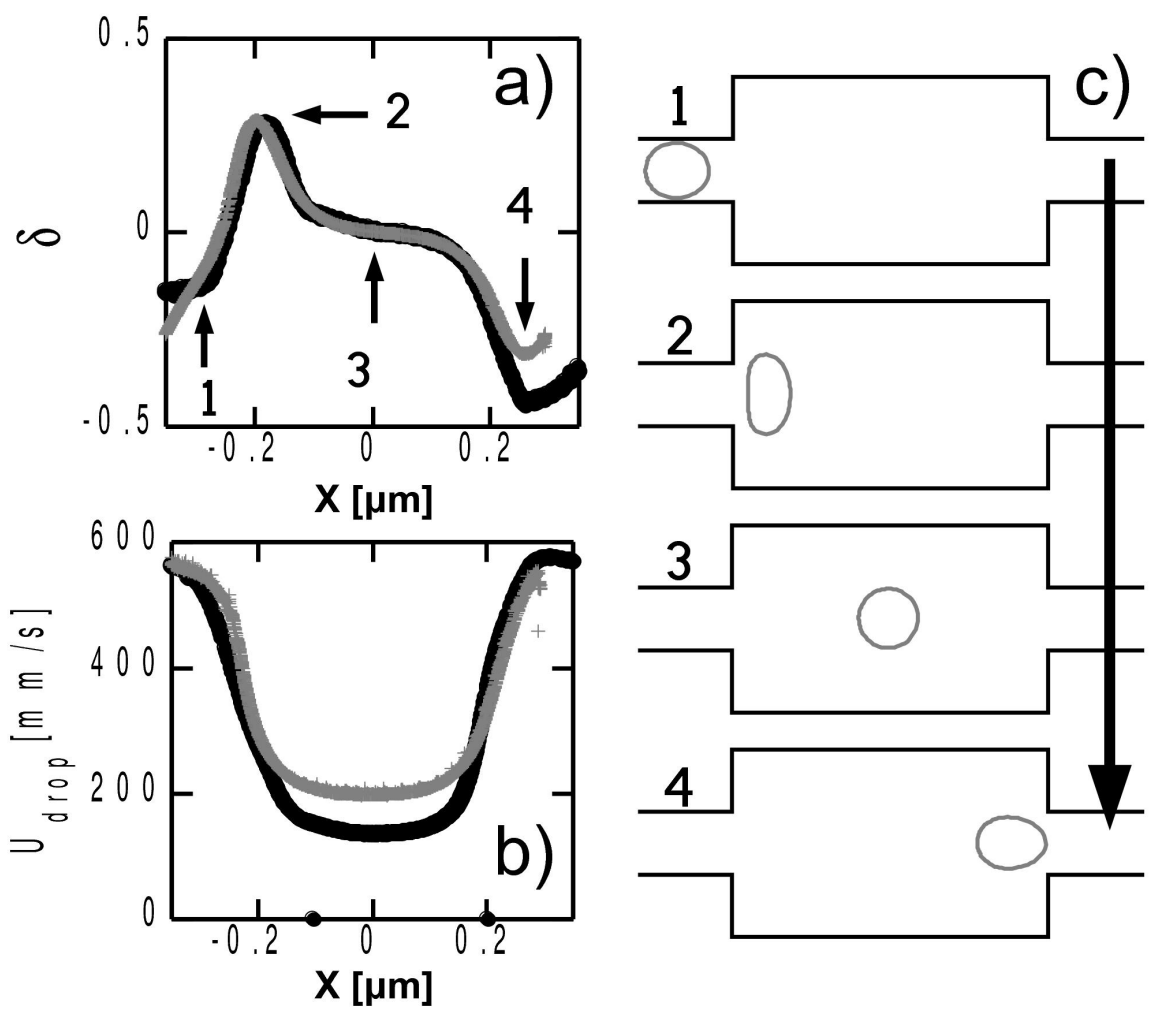

Figure 8.2 (a) Droplet deformation profile $\delta(x)$ extracted experimentally (black), and numerically (gray). Numbers corresponds to the position depicted in panel(c). (b) Speed profile $U(x)$ of the droplet as a function of its position in the channel, same color code applies. (c) Geometrical conformation of the droplet in function of its position in the channel. These contours are created using our numerical model, for a droplet radius $R=47.2 \mu \mathrm{m}$ and a Capillary number $\mathrm{Ca}=3.5 \times 10^{-2}$.

To validate the consistency of the numerical model, we compare the deformation and speed profiles of droplets obtained on-chip to the simulated ones which were obtained using the same conditions of the Capillary number, and the droplet size. In figure 8.2 (a), (b), we show these profiles through the expansion geometry. Experiments and simulations seem to be in good agreement in the region of the droplet maximum deformation (point 2 in figure 8.2 (b)). Divergences between the model and the experiment are however noticeable in the position of 
the maximum deformation. They can be attributed to the error in the resolution of the experimental channel geometry, for instance the smoothing of the corners at the opening, or small variations in the channel width from chip to chip. Larger deviations are seen on the velocity profile that can be attributed to deviations in the channel height originating from the chip fabrication. Despite these variations, the quantitative agreement is satisfactory enough to validate the comparison in between the experiment and the numerical simulations.
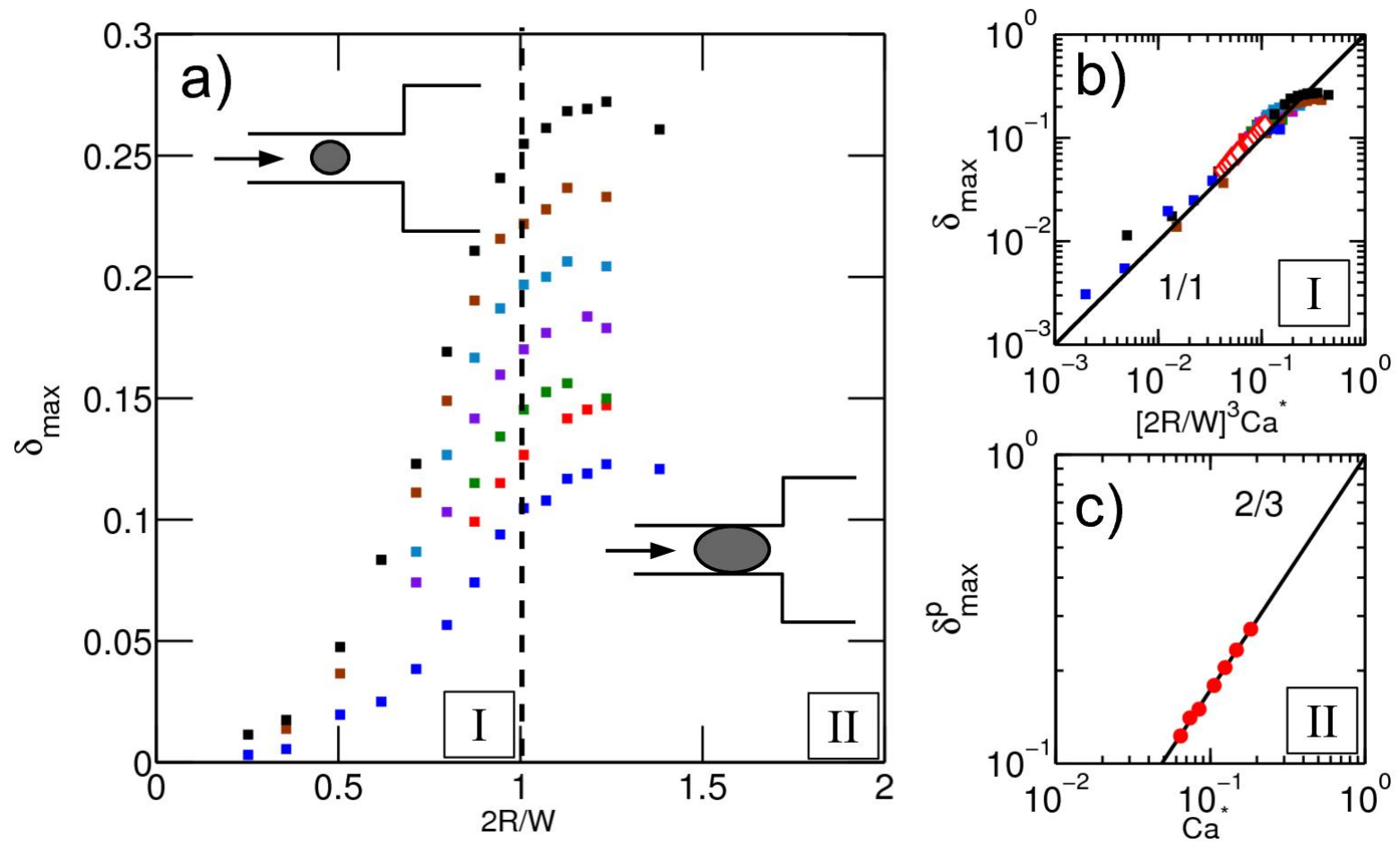

Figure 8.3 (a) The results of the numerical simulation. The values of $\delta_{\max }$ are simulated for droplet with radius $R=10 \mu \mathrm{m}$ to $70 \mu \mathrm{m}$. The change in color shows the different surface tensions used: $\gamma=50 \mathrm{mN} \cdot \mathrm{m}^{-1}(\mathbf{\square}), \gamma=43.5 \mathrm{mN} \cdot \mathrm{m}^{-1}(\square), \gamma=36.7 \mathrm{mN} \cdot \mathrm{m}^{-1}(\square), \gamma=29.2 \mathrm{mN} \cdot \mathrm{m}^{-1}$ (घ), $\gamma=23.9 \mathrm{mN} \cdot \mathrm{m}^{-1}(\varpi), \gamma=19.5 \mathrm{mN} \cdot \mathrm{m}^{-1}(\mathbf{\square}), \gamma=15 \mathrm{mN} \cdot \mathrm{m}^{-1}(\mathbf{\square})$. Two region appears: I the droplet maximum deformation depends on parameters $R$ and $\mathrm{Ca}$; II where $\delta_{\max }$ is constant with $R$. The limit between these two regime is $2 R / W=1$. (b) The rescaling of the data of the region $\mathbf{I}$ according to equation 8.4 . We added here a simulation keeping a constant droplet size $\gamma=35.7 \mu \mathrm{m}(\diamond)$. (c) Droplet maximum deformation at the plateau $\delta_{\max }^{\mathrm{p}}$ (region II) in function of the Capillary number, at constant droplet size $R=61.8 \mu \mathrm{m}$. The black solid line gives the best fit for this trend. 


\section{Numerical results}

The figure 8.3 (a) shows the data obtained with this numerical simulation, by varying the droplet radius and the surface tension, while keeping the droplet speed constant. A plateau region for which the maximum deformation is constant appears for a droplet radius $2 R / W=$ $50 \mu \mathrm{m}$. This size corresponds to a droplet diameter equal to the channel width. From here we define two regions which seems to obey to different scaling. The region (I) that corresponds to the the increase of $\delta_{\max }$ with the droplet size, and a region (II) where $\delta_{\max }$ is a constant in $R$. The deformation in the region (I) can be rescaled (figure 8.3 (b)) according to the modified Capillary number $\mathrm{Ca}^{*}$ and the non-dimensional droplet radius $2 R / W$ in the following way:

$$
\delta_{\max }^{\mathrm{I}}=\left(\frac{2 R}{W}\right)^{3} \mathrm{Ca}^{*}
$$

The value reached by the plateau is different for different surface tensions. We can then extract a scaling of $\delta_{\max }^{\mathrm{p}}$ that only depends on the Capillary number: $\delta_{\max }^{\mathrm{II}} \sim \mathrm{Ca}^{2 / 3}$. See figure 8.3 (c)

\section{Experimental results}

The scalings found above are then tested in various confinement conditions in microfluidic environment. The results are shown on figure 8.4. For each channel height, we gather the deformations obtained for different Capillary number varying from $\mathrm{Ca}=2 \times 10^{-3}$ to $2 \times 10^{-1}$. Here the experimental conditions do limit us in the discussion of the region $\mathbf{I}$. We notice that the experimental droplet deformations are not following the same scaling as found numerically (equation 8.4) as the exponent on the modified Capillary number $C a^{*}$ is found to be smaller than one. Taken individually and without the contribution of the geometrical prefactor, the exponents on the modified Capillary number vary as function of the channel height. For strong confinement where the channel is the closer to the two dimensional model, the exponent is $\sim \mathrm{Ca}^{* 0.9}$, which is close to one. On the other side for non confined droplets, the exponent is reduced to $\sim \mathrm{Ca}^{* 0.67}$, where we find the same exponent as in the previous chapter. See figure 8.4. We can deduce that despite the small channel heights, the droplet deformations in our geometries can not be described by a two dimensional model, and that the increase of the channel height drives us away from this model. 


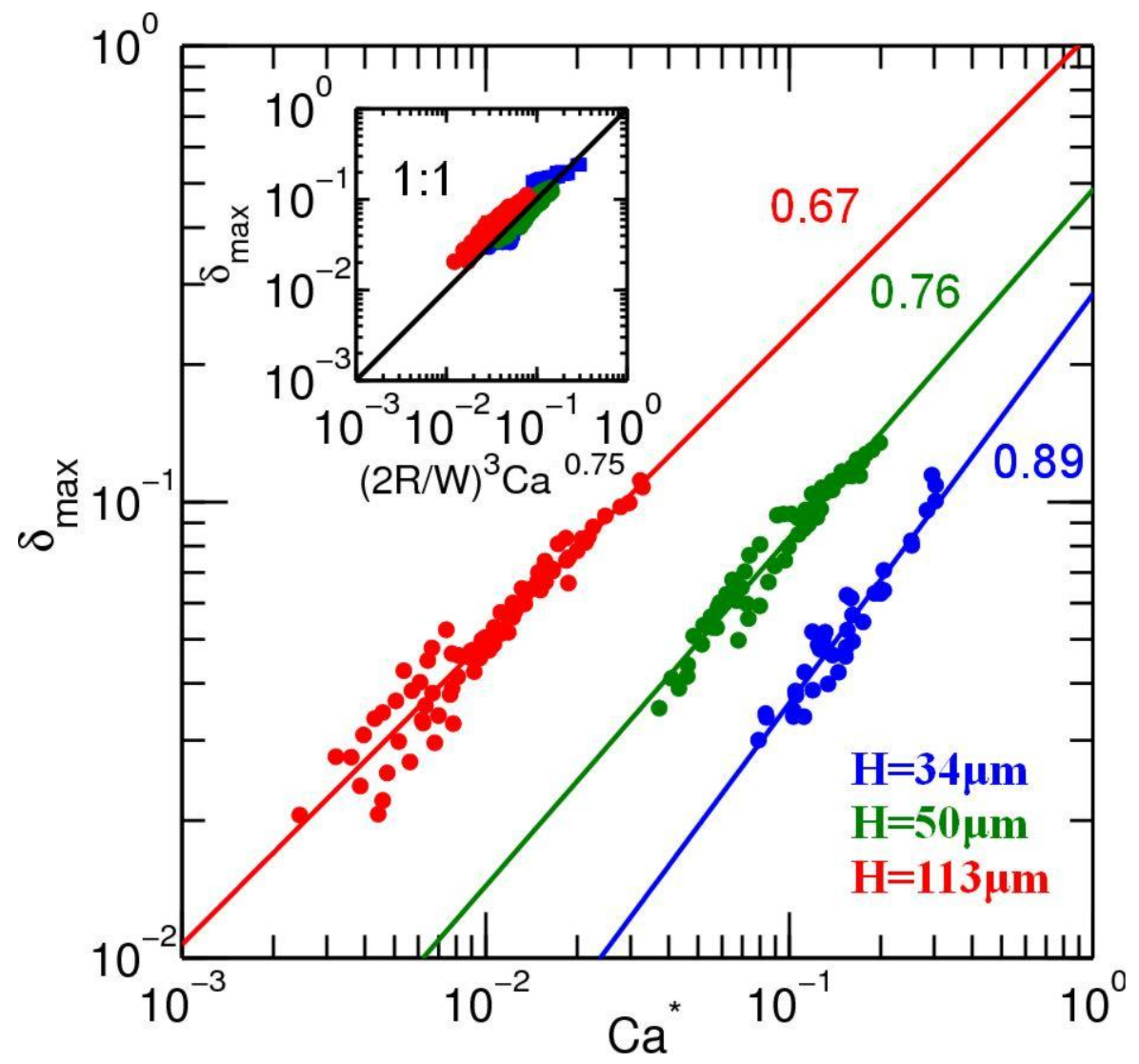

Figure 8.4 The droplet maximum deformation $\delta_{\max }$ obtained experimentally, for the three different channel heights $H=34 \mu \mathrm{m}$ (blue), $H=50 \mu \mathrm{m}$ (green) and $H=113 \mu \mathrm{m}$ (red). The best fit on the modified Capillary number $\mathrm{Ca}^{*}$ of the experimental data that are situated in the region $\mathbf{I}$ is found to vary with the channel height, from $\sim \mathrm{Ca}^{* 0.89}$ for a channel close to the two dimensional condition to $\sim \mathrm{Ca}^{* 0.67}$ for a channel that is completely three dimensional. Inset The same data set rescaled with the geometrical prefactor found for the region $\mathbf{I}$, and for the exponent close to the one found by Cubaud[137]. Note that this is only valid as an approximation.

\subsubsection{Surfactant covered droplets}

We are now interested to test the validity of the scalings found previously in the case of droplet interfaces covered with surfactant molecules. When the droplets are produced with surfactant, one can achieve a stable production of sufficiently large droplets to enter the region II. The experiments are performed in a channel $H=50 \mu \mathrm{m}$ high. The interface is equilibrated along the delay line to a surface tension close to the equilibrium $\gamma e q$. The surface tension is measured on-chip using the method described in the previous chapter. We use the PEG-PFPE molecules and Krytox-NH4 to test the possible effects that differences in the physico-chemical proper- 
ties of the molecules could induce on the scaling of the region $\mathbf{I}$. The figure 8.5, shows the comparison between the data obtained with surfactant (colored triangles), to the data obtained without surfactant (gray symbols) in the same condition of confinement $(\mathrm{H}=50 \mu \mathrm{m})$. The case with the surfactant seem to follow the same scaling law was found without surfactant in the region I. The plateau in the region II is visible for larger droplet sizes. Unfortunately the large uncertainty on the surface tension measured for surfactant-laden interfaces, and the relatively low range of surface tensions available, makes it difficult to confirm the scaling which was found numerically.

The shape and position of the droplets that can be extracted at $\delta_{\max }$ give us a further distinction between the two regimes. The numerical simulation provides comparison between the position and the shape of the two-dimensional interface. We show in figure 8.6, the droplet contour for a set of droplet areas, for surface tensions $\gamma=50 \mathrm{mN} \cdot \mathrm{m}^{-1}$ (figure 8.6 (a)) and $\gamma=15 \mathrm{mN} \cdot \mathrm{m}^{-1}$ figure 8.6 (b)). These profiles show that the deformation occurs at a fixed point close to the opening for droplet size below $2 R / W<1$ (red profiles). This is in accordance with the strong influence of the geometrical prefactor, on the droplet deformation in region I. In contrary for droplet $2 R / W>1$ (blue profiles) the position of droplet maximum deformation $\delta_{\max }$ varies significantly with the droplet size. Namely, the droplet has to completely exit the channel in order to get fully deformed.

\subsubsection{Discussion}

The geometric contribution to the scaling, as well as the appearance of a plateau for the deformation of large droplets, points to a strong influence of the flow profile that arises from the droplet confinement. We compare two types of droplet transport in a microfluidic channel. The first transport described in region $\mathbf{I}$, is a dispersed flow where the continuous phase is free to circulate around the droplet. In this case, when the droplet reaches the expansion, the flow profile of the continuous phase is modified by the droplet size and changes the viscous stress at the interface. In the region II, the circulation of the droplet is better described by a plug flow, where only a lubrication layer between the wall and the interface exists [41, 118]. In this case the obstruction of the channel by the droplet imposes a fixed condition on the speed of the continuous phase which has a speed larger than the droplet. As a consequence the droplet maximum deformation occurs much later in the chamber. Here the spacing between the droplet and the wall is large. The size of the droplet has less influence on the flow profile of the continuous phase around the droplet. The flow conditions being fixed, the surface tension 


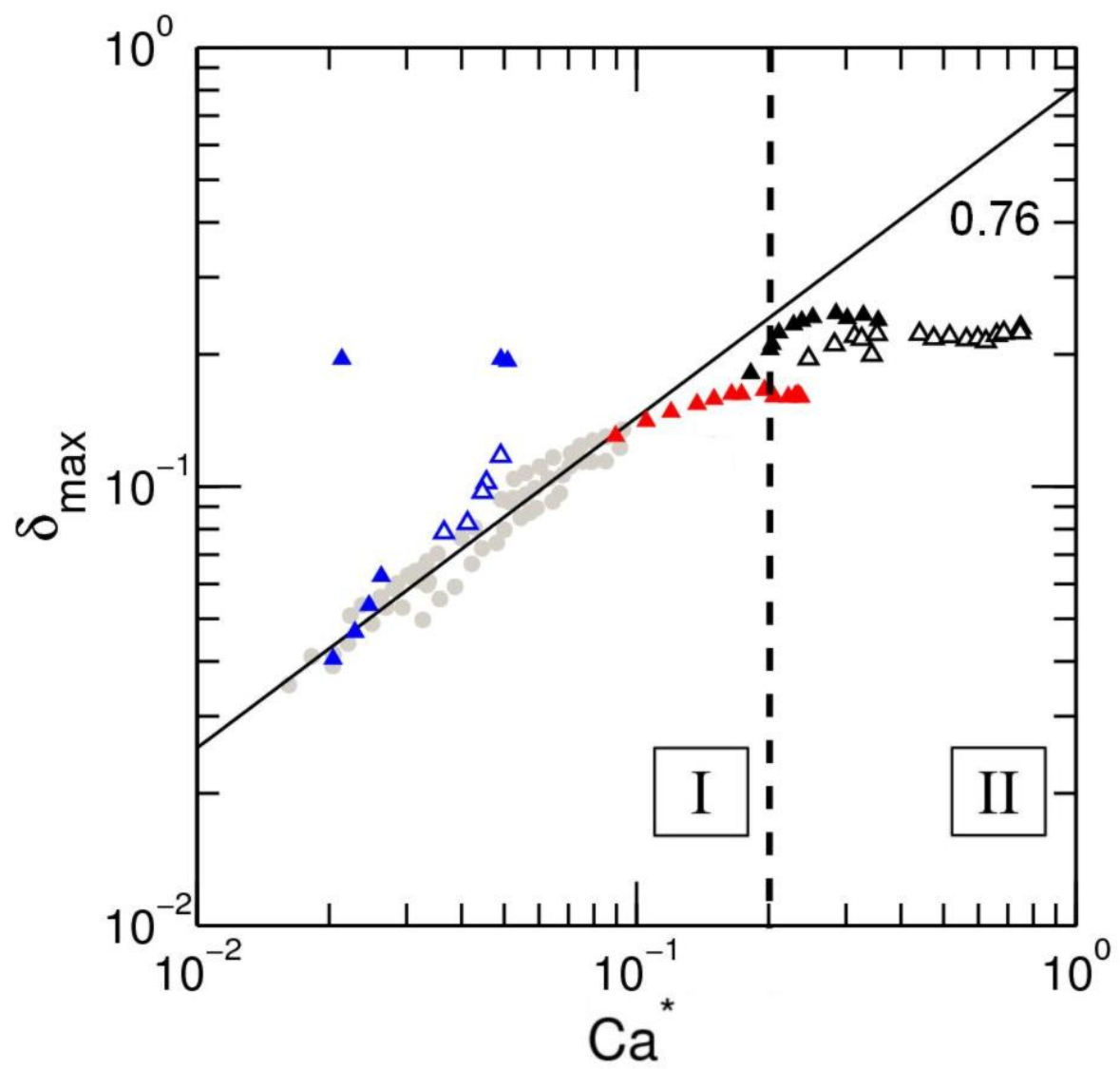

Figure 8.5 The droplet maximum deformation $\delta_{\max }$ in presence of surfactant, for the two different molecules PEG-PFPE $(\triangle)$ and Krytox-NH4 (ム). The different concentrations with respective surface tension at equilibrium are; $C_{\mathrm{PEG}-\mathrm{PFPE}}=1.3 \times 10^{-2} \mathrm{~mol} \cdot \mathrm{m}^{-3}: \gamma_{\mathrm{eq}}=$ $19 \mathrm{mN} \cdot \mathrm{m}^{-1}(\triangle), C_{\mathrm{PEG}-\mathrm{PFPE}}=1.3 \mathrm{~mol} \cdot \mathrm{m}^{-3}: \gamma_{\mathrm{eq}}=1.32 \mathrm{mN} \cdot \mathrm{m}^{-1}(\triangle), C_{\mathrm{K}-\mathrm{NH}_{4}}=$ $2.6 \times 10^{-1} \mathrm{~mol} \cdot \mathrm{m}^{-3}: \gamma_{\mathrm{eq}}=23 \mathrm{mN} \cdot \mathrm{m}^{-1}(\Delta), C_{\mathrm{K}-\mathrm{NH}_{4}}=1.3 \mathrm{~mol} \cdot \mathrm{m}^{-3} \gamma_{\mathrm{eq}}=5 \mathrm{mN} \cdot \mathrm{m}^{-1}$ $(\boldsymbol{\Lambda}), C_{\mathrm{K}-\mathrm{NH}_{4}}=6.5 \mathrm{~mol} \cdot \mathrm{m}^{-3} \gamma_{\mathrm{eq}}=4 \mathrm{mN} \cdot \mathrm{m}^{-1}(\mathbf{\Lambda})$. The grey symbols represent the data obtained without surfactant, for the same channel height $H=50 \mu \mathrm{m}$, in background for better comparison. The results obtained with the surfactant are consistent to the geometrical scaling found before. The slope of the black line is the best fit found for the Capillary number. We represent the separation between the regions I and II by a dashed line.

becomes the only parameter influencing the deformation, explaining the sole dependence on the Capillary number.

It is to be noted that a power law $\left(\mathrm{Ca}^{0.74}\right)$ was found by Cubaud [137] in his description of the elongation of a viscous droplet in a three dimensional square straight channel. Experimentally a good collapse of the droplet maximum deformation is found for all the channel heights data when the data are rescaled by the expression $\delta_{\max } \sim(2 R / W)^{3} \mathrm{Ca}^{0.75}$. See the inset of the figure 8.4 . This behavior is probably not universal but the two cases possibly share common 

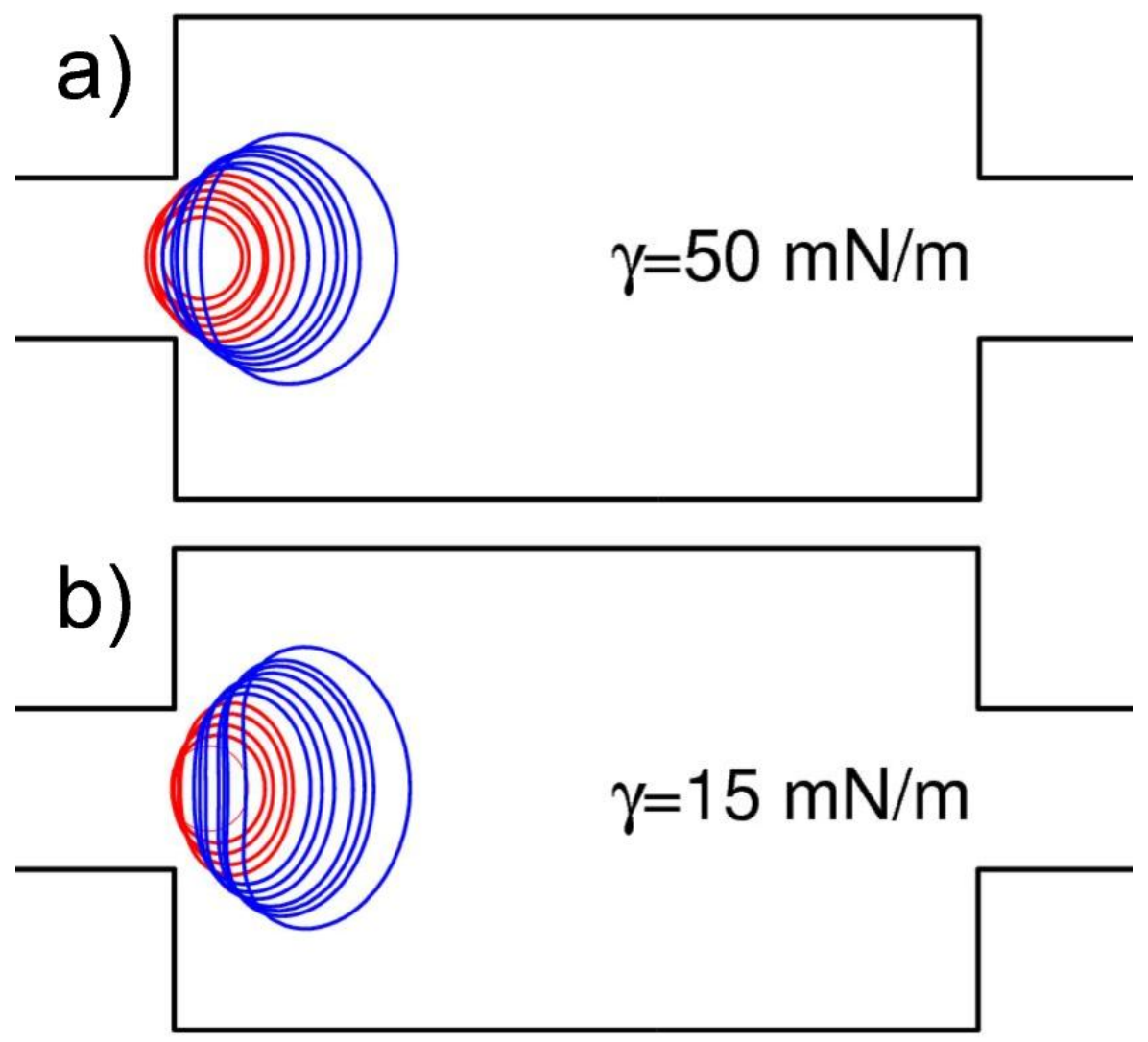

Figure 8.6 Simulated droplet contour, and position at its maximum deformation. The red contour represents the droplet maximum deformation in the first regime $2 R / W<1$. The blue contour represent the deformation in the second regime $2 R / W>1$ where the deformation is independent from the droplet size. Two surface tension are simulated: $\gamma=50 \mathrm{mN} \cdot \mathrm{m}^{-1}$ (a) and $\gamma=15 \mathrm{mN} \cdot \mathrm{m}^{-1}$ (b).

features that lead to close dependency of the Capillary number.

The two scalings obtained in our analysis clearly express the dependence on the interfacial conditions, by the means of the modified Capillary number $\mathrm{Ca}^{*}$, and the influence of the geometry with the prefactor $[2 R / W]^{3}$. The modification of the dependence on the modified capillary number is interpreted as the result of further deformations of the interface that are occurring in the z-direction. In our experimental conditions, the interface can not be reduced to a planar circular contour, and this is particularly true for channel heights that are of the order of the droplet diameter. As a consequence there is a non negligible part of the deformation that occurs out of plane. This deformation is not accessible with our experimental method, and can not be quantified. Further full three dimensional simulation are being performed to 
address this question.

In summary of this part, we have seen that the droplet deformation in microchannels strongly depends on the droplet confinement in the channel before the expansion. We show that in the geometry of a planar sudden expansion the droplet maximum deformation $\delta_{\max }$ follows two regimes linked to its confinement in the microfluidic channel. A first regime occurs when the droplet diameter is smaller than the channel and is characterized by a dependence of the maximum deformation on the Capillary number and the size of the droplet. A second regime occurs for droplets larger than the channel size where the maximum deformation depends on the Capillary number. A qualitative agreement could be found between the implementation of two dimensional numerical simulations and the experiments, for the description of these regimes. However quantitatively the experimental values are found to deviate from a two dimensional description of the flow with Darcy equation, as the height of the channel considered is getting larger. In the following section we compare the analysis of the droplet deformation with a three dimensional model.

\subsection{Deformation of spheroidal droplets in elongational flow profile}

We have seen in the previous part that the droplet deformation in a sudden planar expansion can not be quantitatively described by a two dimensional simulation. In this part we study the droplet deformation in the elongational flow profile while re-entering the channel after the deformation chamber. In these flow conditions, we compare our results with a previously established model[109] that links the deformation profile to the gradient of the flow velocity and the surface tension. This model is applicable for droplets which shapes that can be approximated by a spheroid, making this analysis essentially three dimensional. We want by applying this model to our experimental conditions, to estimate the deviation from a three dimensional description.

In the limit of small deformations, the droplet deformation can be described by the solution of the Stokes equation, for nearly spherical geometries. The flow profile inside and outside the droplet is approximated to be purely extensional, and the deformation from the original shape is small, typically below $15 \%[111]$. From early works of Taylor [110] the relaxation of 
a spheroidal droplet in a non-inertial flow of a newtonian fluid is described as follows:

$$
\frac{\partial \delta}{\partial t}=\frac{5}{2 \dot{\eta}+3} \dot{\epsilon} \tau_{\text {visc }}^{2}-\delta \tau_{\text {visc }}
$$

where $\delta$ is the droplet deformation, $\dot{\eta}=\eta_{\text {in }} / \eta_{\text {out }}$ is the ratio between the disperse phase viscosity $\eta_{\text {in }}$ to the continuous phase viscosity $\eta_{\text {out }}, \dot{\epsilon}=d u / d x$ is the velocity gradient in which the droplet flows. The viscous characteristic time $\tau_{\text {visc }}$ depends on the viscosity of both the fluid and on the surface tension, and is defined as:

$$
\begin{aligned}
\tau_{\text {visc }} & =\frac{\alpha \eta_{\text {in }} R_{0}}{\gamma} \\
\text { with } \quad \alpha & =\left[\frac{(2 \dot{\eta}+3)(19 \dot{\eta}+16)}{40(\dot{\eta}+1)}\right]
\end{aligned}
$$

$\alpha$ is an effective viscosity derived by Taylor for the study of the viscosity of a fluid containing deformable spheres[131]. $R_{0}$ is the radius of the droplet at rest.

In the case of stationary boundary conditions at the interface, the relaxation of the droplet is described by the second term of the right side of the equation 8.5 . The relaxation to an equilibrium shape is an exponential decay:

$$
\delta(t)=\delta(0) \exp -t / \tau_{\text {visc }}
$$

where $\delta(0)$ is the deformation of the droplet at initial time $t=0$. In our geometry we recover the conditions of an uniaxial elongational in the second part of our deformation chamber. In this channel part, the convergence of the streamlines due to the sudden constriction makes our situation adequate to apply the analytical description developed above. We expect to retrieve from the different channel heights, a qualitative deviation from this model due to the deviation of the droplet shape from an overall spherical shape.

In our geometry, the presence of a background flow requires the analysis through the full equation 8.5 . This is particularly true in the presence of rapid changes in the velocity, where the quantity $d u / d x$ becomes non negligible. We can here refer to the velocity profiles presented in figure 8.2 (b) to estimate the magnitude of this term. In the study of Cabral et al.[109] the expression for the droplet deformation in an extensive flow is derived in a more usable form:

$$
\alpha \eta_{\text {in }}\left(\frac{5}{2 \dot{\eta}+3} \frac{d u}{d x}-u \frac{\partial \delta}{\partial t}\right)=\gamma\left(\frac{\delta}{R_{0}}\right)
$$


The above quantities can be extracted from our experimental microfluidics setup. This form allows us to establish a linear relation between the left side of the equation and the right side based on the surface tension only. In our experiments we approximate the value of the velocity profile by the velocity profile of the droplet: $u \sim U_{\text {drop. }}$ The velocity and deformation profiles are fitted with higher order polynomials, respectively $\mathrm{O}(6)$ and $\mathrm{O}(5)$, in order to smooth the experimental data. The gradients $d u / d x$ and $\partial \delta / \partial x$ are the derivatives of these polynomial expressions. The fits and the resulting gradients can be seen in figure 8.7 (a)-(d). In our definition of $\delta$ the deformation prolate in the x-direction results in a negative value of $\delta$. In order to perform the treatment, the absolute value of $\delta$ is used.

In figure 8.7 (e) is shown one example of a the so-called Taylor plot that represents the graphical resolution of the equation 8.9 . We superpose on this plot a line with a slope 49.5, which is the surface tension in this case. We see that the Taylor plot can be approximated by a linear behavior for the small values of $\delta$ which corresponds to the regions where the droplet is approaching the channel. See figure 8.7 (b). For larger deformations, where the droplet actually starts entering the channel, the velocity gradient of the droplet starts to decrease, see figure 8.7 (c). Therefore the conditions of an elongational flow conditions at higher values of $\delta / R_{0}$ are not respected anymore, which explains the deviation from the linear behavior. In the following study, we will use the linear part of this plot to test the validity of this model. The region where the flow can be considered as elongational is marked by the vertical red lines on the figure 8.7 (c) and (d).

We proceed in the following way. For each channel depth $H=34,50$ and $113 \mu \mathrm{m}$, three droplet deformation profiles corresponding to three different surface tensions, in a range from $\gamma=49.5$ to $10.6 \mathrm{mN} \cdot \mathrm{m}^{-1}$, are used.

Figure 8.8 presents the same graphical resolution of the equation 8.9 for the three channel depths available. We draw the Taylor plot for three different surface tensions, each for two deformation profiles. The surface tension values, $\gamma$, measured previously on chip are used to draw the linear dependence between the two sides of the equation 8.9 , represented as a colored solid line. The comparison between the resulting straight line and the Taylor plot allows us to estimate the precision of the measurement. When discrepancies are found between these two values, a fitting of the curve is made to determine the range of surface tensions that would be predicted by the Taylor analysis. The results of these comparisons are displayed in table 8.2

In Table 8.2, are presented the surface tensions $\gamma$ as measured with pendent drop method. In addition we compare these results with the graphical resolution of the equation 8.9. We use the notation $\gamma_{\mathrm{H}}$ with $H$ the channel depth to represent the best fit found in the linear region of 

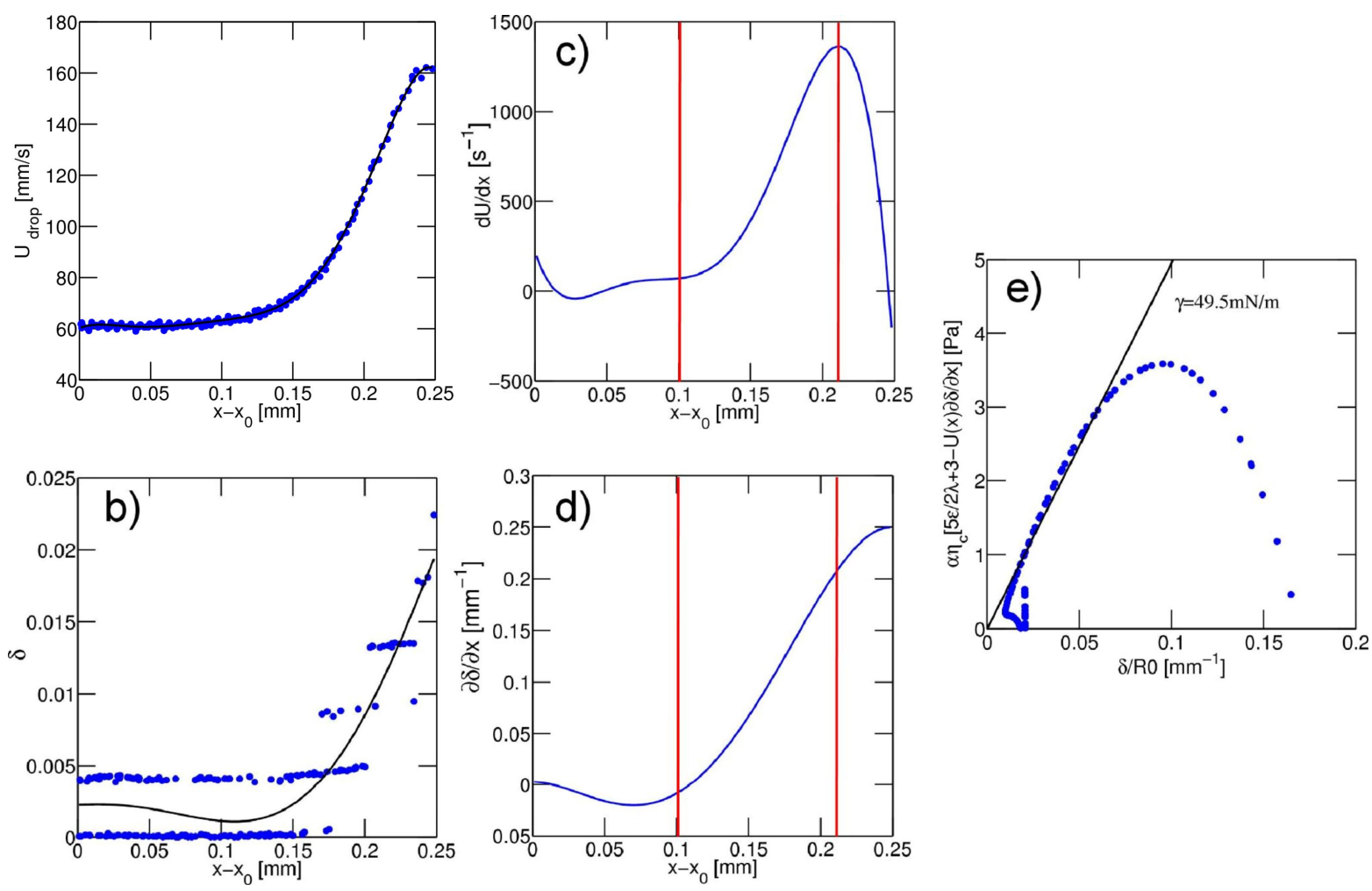

Figure 8.7 Data set obtained for the deformation of a millipore droplet in oil and used for the computation of the Taylor plot. (a) Velocity profile of the droplet re-entering the channel at the end of the deformation chamber. (b) Deformation profile of the droplet in the same region. (c) First derivative of the velocity profile $d u / d x$. (d) First derivative of the deformation relative to the position $\partial \delta / \partial x$. (e) The so-called Taylor plot, obtained in this region. The black line represents the linear relationship between the two members of the equation 8.9 . Here for the surface tension $\gamma=49.5 \mathrm{mN} \cdot \mathrm{m}^{-1}$. The red line limits the region where the flow profile is considered as elongational, and where the linear relation on figure (e) is defined.

the Taylor plot. Three surface tensions are presented for each channel depth.

As expected the graphical resolution of the equation 8.9 gives the most consistent results for an unconfined droplet $(\mathrm{H}=113 \mu \mathrm{m})$. In this case the channel top and bottom walls are sufficiently far away to approximate the droplet shape as a spheroid. In more confined case, ( $H=50 \mu \mathrm{m}$ and $H=34 \mu \mathrm{m})$ the droplet shape is closer to a cylinder or a "pancake shape" However, we notice that the precision on the surface tension value extracted is strongly linked to the magnitude of the droplet deformation. Hence a better agreement is found for the low surface tensions.

This part shows that the application of a three dimensional model to the droplet deformation in 

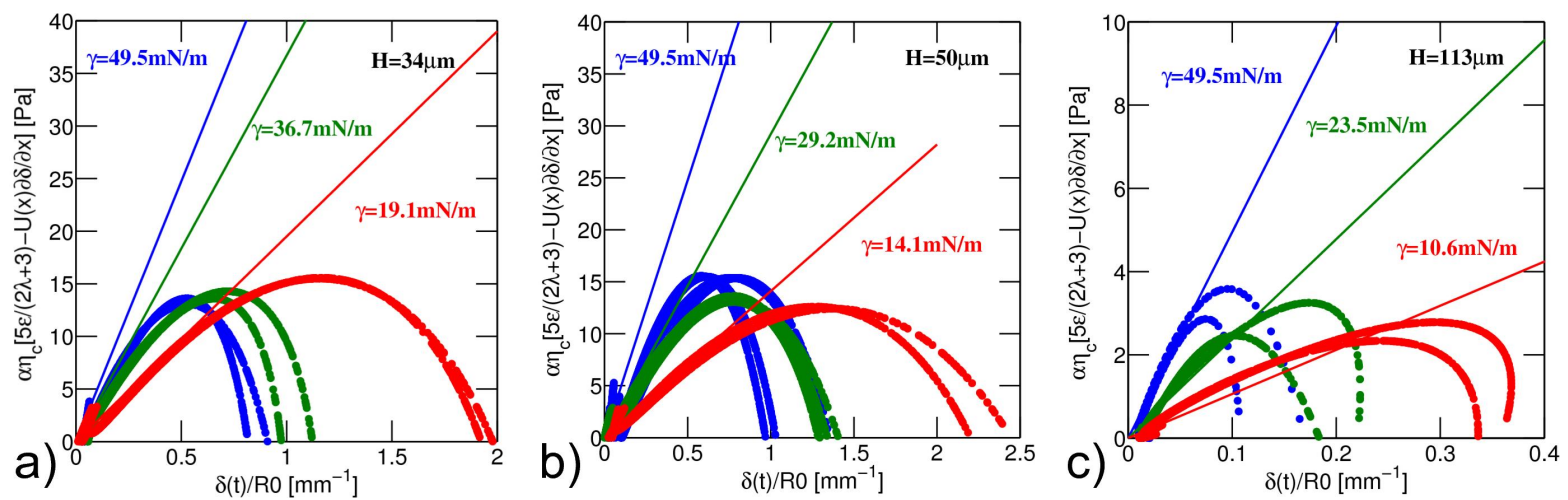

Figure 8.8 Taylor plots calculated from the deformation of droplets with different water/ethanol mixtures. The three figures corresponds to three different channel depths: (a) $H=34 \mu \mathrm{m}$; (b) $H=50 \mu \mathrm{m}$; (c) $H=113 \mu \mathrm{m}$, each color corresponds to a surface tension $\gamma$ that has been measured in pendant drop method. This value is also used to draw the linear relation, that is compared to the linear part of the Taylor plot.

\begin{tabular}{cccc}
\hline$\gamma \mathrm{mN} \cdot \mathrm{m}^{-1}$ & $\gamma_{113} \mathrm{mN} \cdot \mathrm{m}^{-1}$ & $\gamma_{50} \mathrm{mN} \cdot \mathrm{m}^{-1}$ & $\gamma_{34} \mathrm{mN} \cdot \mathrm{m}^{-1}$ \\
\hline \hline 49.6 & $50 \pm 2$ & $28 \pm 8$ & $32 \pm 3$ \\
\hline 36.7 & \multicolumn{3}{c}{$30 \pm 5$} \\
\hline 29.2 & $25 \pm 5$ & \\
\hline 23.9 & $28 \pm 3$ & $17 \pm 3$ \\
\hline 19.5 & \multicolumn{3}{c}{$13 \pm 2$} \\
\hline 14.1 & & \\
\hline 10.6 & $15 \pm 5$ &
\end{tabular}

Table 8.2 Comparisons between the surface tensions measured in pendant drop method $(\gamma)$ and the surface tension extracted from the the graphical resolution of the equation 8.9 in figure 8.8 . The surface tension reads as follows $\left(\gamma_{\mathrm{H}}\right)$ where $H$ is the channel depth considered.

our microfluidics channel is valid, in the limit of detectable droplet deformations. In addition, the region where the conditions of elongational flow is satisfied is small in our channel. Thus, the results extracted for small channel heights lack of reliability due to the large deviation of the droplet shape from a spheroid in this case. The reliability can be compared to a previous studies that show deviation in the measured surface tension of the order of $10 \%$ for droplet size $20 \%$ larger than the channel[109]. 


\subsection{Droplet relaxation in inertial regime}

In this part we focus our attention on the relaxation of the droplet which occurs between the points defined previously as the droplet maximum deformation $\delta_{\max }$, and the rest circular shape of the droplet resting state approximatively in the center of the chamber. We show that under certain flow conditions the relaxation profile of the droplet exhibits a non-monotonic decay, which is interpreted as presence of inertial effects. Although being uncommon at these length scales, inertial effects have already been studied in microfluidics[138]. In this case, the maximum of deformation is followed by one or two visible oscillations, see figure 8.9. We test a model of deformation which describes the free oscillation of an interface, and the damping of the oscillation by dispersion of the energy in the fluid surrounding the interface. The first mention of the relaxation of an interface through inertial process is made by Rayleigh[139]. He establishes the theoretical formulation on an oscillating jet interface through its surface tension and the viscosity of the surrounding phases. Since then this model has been used to describe different kinds of periodic deformations occurring at interfaces [140-142]

\subsubsection{Model}

In order to describe the oscillations of the interface, we consider the system being completely described by one variable which is the droplet deformation $\delta$. The low amplitude of the droplet deformations allows the description of the process in the range of small perturbations. Therefore our interface is assumed to behave like an elastic material and can be described by a harmonic oscillator. The presence of viscous dissipation from the surrounding liquids, gives the damping component. The equation of damped oscillation is described classically as following:

$$
\frac{d^{2} \delta}{d t^{2}}+\zeta \frac{d \delta}{d t}+\delta \omega_{0}^{2}=0
$$

where $\omega_{0}$ is the frequency of the system and $\zeta$ its damping ratio.

It is possible to recover this differential equation from the momentum equation of the Navier-stokes equations (equation 4.5), and was demonstrated in the study of the self excited oscillation of a droplet in electrowetting [142]. Here the characteristic velocity is given by the amplitude $\delta$ of the deformation of the droplet $U \sim \delta / \tau$ where $\tau$ a characteristic time, leading to $\partial U / \partial t \sim \delta / \tau^{2}$ and $U \vec{\nabla} U \sim \delta^{2} /\left(R \tau^{2}\right)$. This last term goes to 0 if the deformation $\delta$ is small in comparison to the droplet size $R$. In addition the pressure term can be described by the Laplace 
equation linearized for the small deformations $\nabla P \sim \gamma / \delta \cdot \delta / R^{2}$, and the viscous dissipation term is given as $\eta \nabla^{2} \mathbf{U}$. The equation 8.10 can be rewritten as:

$$
\frac{d^{2} \delta}{d t^{2}}+\frac{1}{O h} \frac{d \delta}{d t}+\delta \omega_{0}^{2}=0
$$

Where the term $O h$ is the Ohnesorge number defined as following:

$$
O h=\frac{\eta_{\text {out }}}{\sqrt{\rho_{\text {in }} \gamma R}}
$$

where $\eta_{\text {out }}$ is the viscosity of the continuous phase and $\rho_{\text {in }}$ the density of the droplet phase. $\omega_{0}$ is the frequency of the free oscillations, that is written:

$$
\omega_{0}=\sqrt{\frac{\gamma}{\rho R^{3}}}
$$

The general solution of the such an equation can be found as:

$$
\delta(t)=A e^{-\frac{t}{\tau_{1}}} \cos \left(t / \tau_{2}+\phi\right)+\epsilon
$$

where $A$ is the amplitude of the oscillations, $\tau_{1}$ is the envelope of the decay in amplitude of the oscillations, $\tau_{2}$ is the period of the oscillations, $\phi$ is a phase shift, and $\epsilon$ an offset from the zero value. The presence of a flow in the deformation chamber imposes a residual stress onto the interface, which depends on the Capillary number which imposes $\epsilon>0$. We use the characteristic times $\tau_{1}$ and $\tau_{2}$ to characterize the decay of the oscillations, see figure 8.9 (a), and the droplet frequency. Usually the value of $\tau_{2}$ is averaged over two or three periods visible during the relaxation process. From the period $\tau_{2}$ we extract the frequency of the oscillations.

$$
\omega=\frac{1}{\tau_{2}}
$$

A typical droplet oscillation profile can be seen in figure 8.9. This figure shows an experimental relaxation profile (•), performed at typical Reynolds numbers $R e>10$ and the best fit obtained by applying the equation 8.14 (black solid line). The additional dashed lines represent the amplitude decay, given by the parameter $\tau_{1}$, and the doted line is the equilibrium to which the droplet relaxation converges. The model shows a good agreement with the experimental data, which proves the reliability of the extracted parameters. 

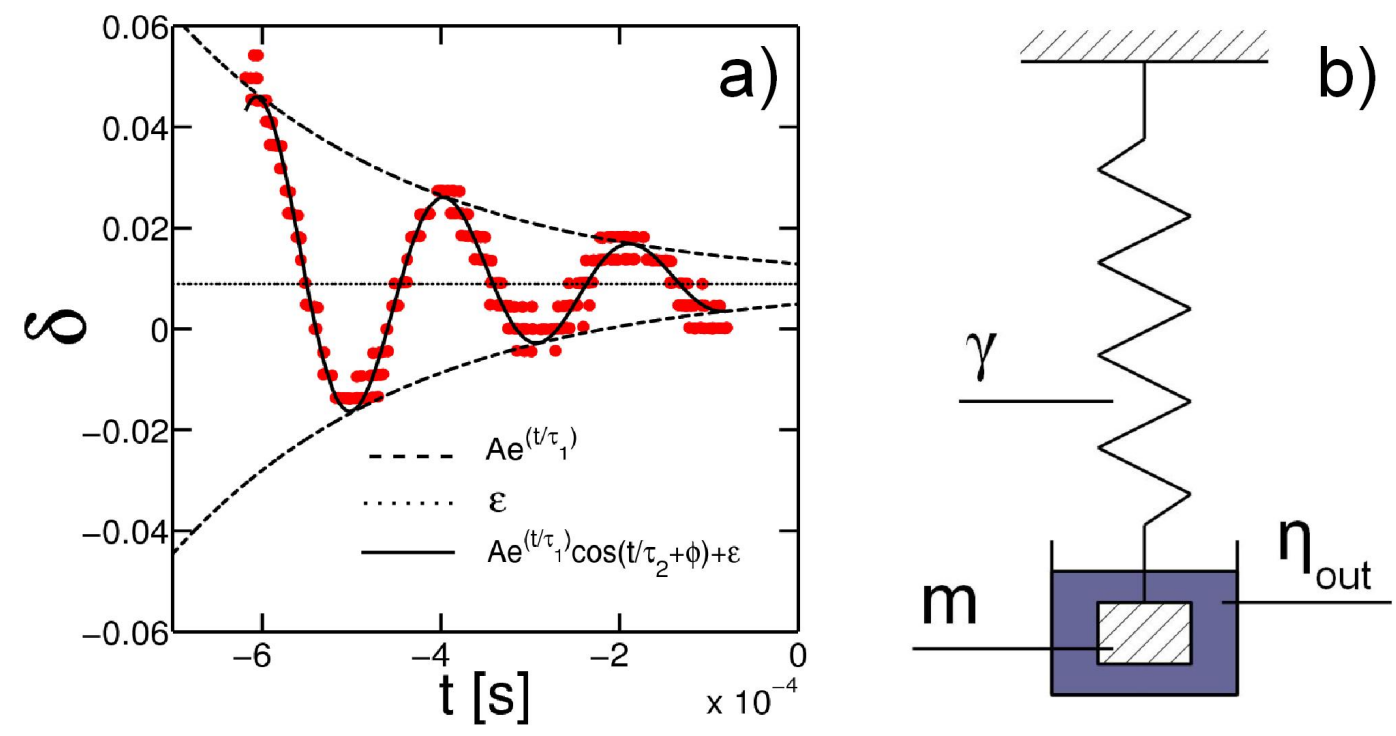

Figure 8.9 (a) The non-monotonic relaxation of the droplet in inertial regime. The experimental data are acquired for a system Water/Oil + Krytox-H concentration $1.3 \times 10^{-2} \mathrm{~mol} \cdot \mathrm{m}^{-3}$. The experimental data can be seen in red. The black solid curve is the result of the fitting of experimental data with the model described in equation 8.14. The dashed line depicts the dissipative term of the model only, and the doted line the offset of the relaxation. (b) Analogy of the oscillation of a droplet with a mass $m=\rho_{\text {in }} V$ and a surface tension $\gamma$ with a damped oscillator, where the viscous dissipation is proportional to the viscosity of the external fluid $\eta_{\text {out }}$.

\subsection{Experimental results}

\subsubsection{Dissipative component of the oscillations}

The value of $\tau_{1}$ is the time scale linked to dissipation of the energy brought to the interface during the deformation. For purely viscous dissipations this quantity has to be related to the typical viscous time scale $\tau_{\text {visc }}$ defined above. The values of $\tau_{\text {visc }}$ can be computed for a typical droplet size $R=50 \mu \mathrm{m}$, and surface tensions $\gamma$ ranging from $\gamma=5-50 \mathrm{mN} \cdot \mathrm{m}^{-1}$, leading to a range of values of $\tau_{\text {visc }}=10^{-5}-10^{-6} \mathrm{~s}$. The values of $\tau_{1}$ extracted from the experiments are situated roughly one order of magnitude higher $\tau_{\text {visc }}$, see figure 8.10. In relaxation processes one can expect the dissipation to be at least dominated by the viscous relaxation $\tau_{\text {visc }}$. Therefore, in presence of an additional dissipative term, the damping characteristic time should be smaller than $\tau_{\text {visc }}$.

As demonstrated previously in presence of a non-stationary flow around the droplet, it is 


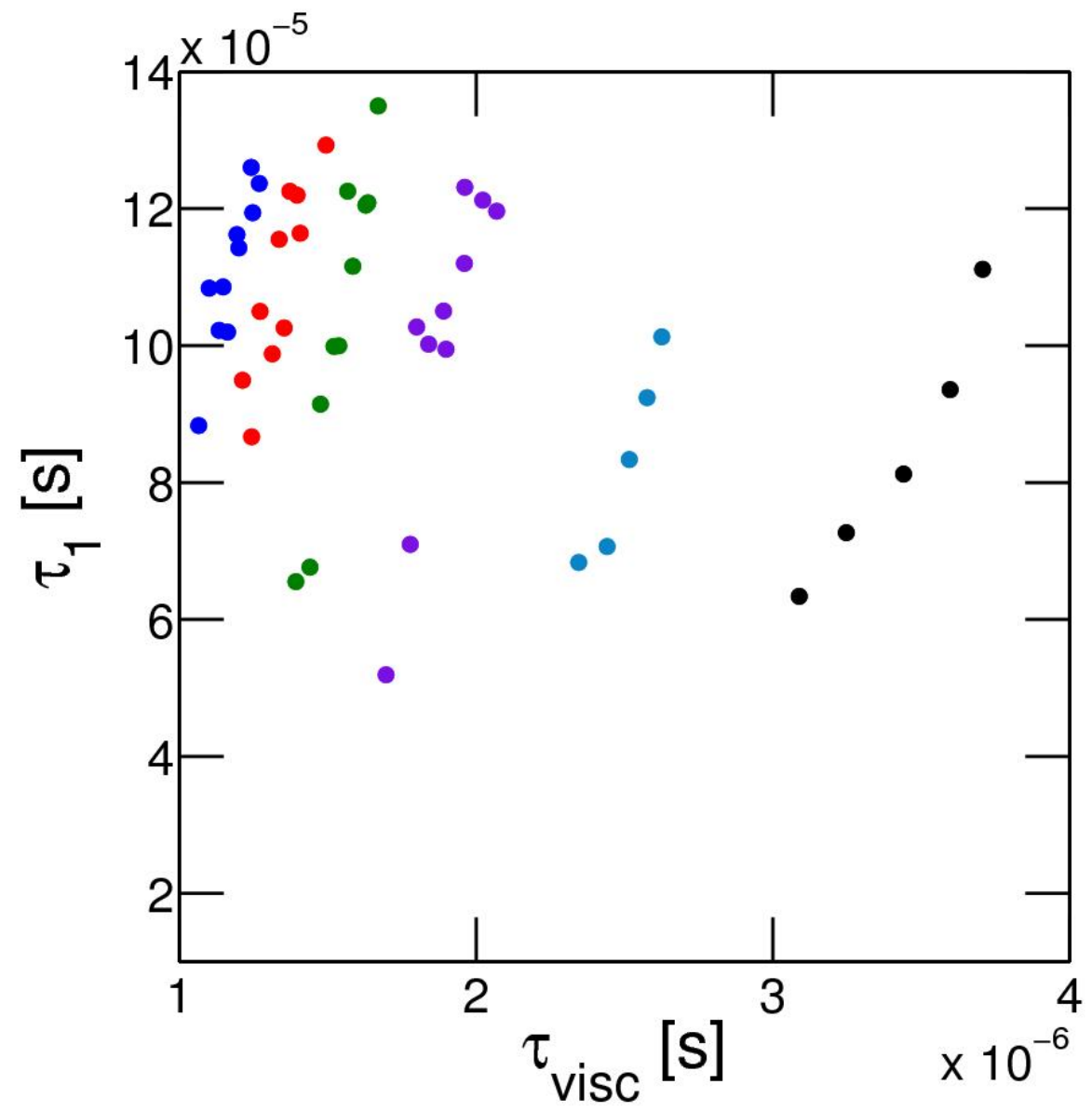

Figure 8.10 Extracted relaxation time $\tau_{1}$ from the experiments compared to the typical viscous dissipations time scale $\tau_{\text {visc. }}$. The fact that $\tau_{1}>\tau_{\text {visc }}$ could be interpreted as the presence of external hydrodynamic forces due to the confinement. The color code corresponds to different surface tensions: $\gamma=49.5 \mathrm{mN} \cdot \mathrm{m}^{-1},(\bullet) ; \gamma=43.5 \mathrm{mN} \cdot \mathrm{m}^{-1},(\bullet) ; \gamma=36.7 \mathrm{mN} \cdot \mathrm{m}^{-1},(\bullet)$; $\gamma=29.2 \mathrm{mN} \cdot \mathrm{m}^{-1},(\bullet) ; \gamma=19.5 \mathrm{mN} \cdot \mathrm{m}^{-1},(\bullet) ; \gamma=14.5 \mathrm{mN} \cdot \mathrm{m}^{-1},(\bullet)$.

necessary to consider the full equation 8.5 for the resolution of the deformation dynamics. Therefore, the droplet relaxation is subject to the influence from the presence of additional hydrodynamic forces that are induced by the confinement. Studies of the droplet circulation in a cylindrical tube have shown that despite the presence of a thin lubrication layer, interactions with the wall can arise in the form a drag forces[143, 144]. The direct consequence on the droplet relaxation is that the dissipation of energy is not anymore limited to viscous dissipation in the droplet phase but also in the surrounding fluids. Theses forces have been found to be the cause of lags in the droplet relaxation[109, 145]. This interpretation is consistent with the results of the studies of the droplet deformation above, that show a strong influence of the 
droplet confinement on the deformation.

\subsubsection{Droplet oscillations}

We use the analogy between the mechanics of a Hookian spring described above and the equation 8.11 derived previously to describe our system. The behavior of the interface can be treated in an analogous way in microfluidics by adapting the droplet volume term $R^{3}$. In microfluidics the projected droplet diameter is large compared to the channel height $2 R \sim$ $100 \mu \mathrm{m}$. To calculate the mass of the droplet we approximate the geometry of the confined droplet inside the channel as a cylinder with a volume $V=A H$, where $A$ is the projected area, and $H$ the depth of the channel. In our experiments the channel depth $H$ is fixed to $H=50 \mu \mathrm{m}$.
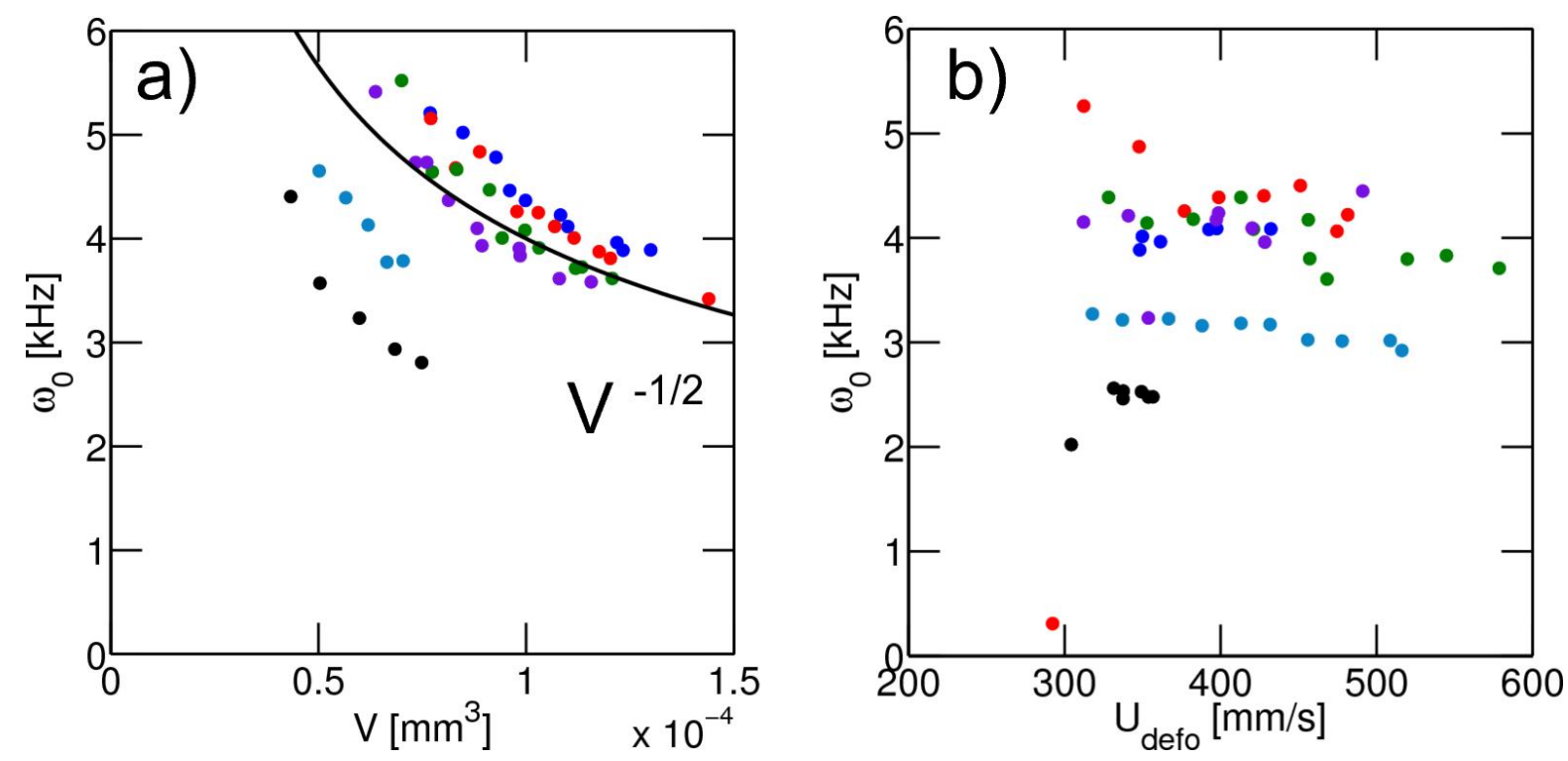

Figure 8.11 Experimental data showing the dependence of the oscillation frequency on the surface tension and the size of the droplet. (a) Dependence of the frequency on surface tension and (b) dependence of the frequency on the speed of the droplet. For the three parameters which are tested, we see a net dependence on the droplet size and the surface tension, which is consistent with the model described by the equation 8.13 . The color code corresponds to different surface tensions: $\gamma=49.5 \mathrm{mN} \cdot \mathrm{m}^{-1},(\bullet) ; \gamma=43.5 \mathrm{mN} \cdot \mathrm{m}^{-1},(\bullet) ; \gamma=$ $36.7 \mathrm{mN} \cdot \mathrm{m}^{-1},(\bullet) ; \gamma=29.2 \mathrm{mN} \cdot \mathrm{m}^{-1},(\bullet) ; \gamma=19.5 \mathrm{mN} \cdot \mathrm{m}^{-1},(\bullet) ; \gamma=14.5 \mathrm{mN} \cdot \mathrm{m}^{-1}$, $(\bullet)$. The black solid line represent a power law $V^{-1 / 2}$ expected from equation 8.13

In figures 8.11, we present the measured droplet frequencies, in function of the two parameters of the droplet that are the droplet size (a) and speed (b). From this experiment we 
can see that $\omega$ is not a function of the speed of the droplet, but depends on its size and surface tension, in accordance with the model defined above.

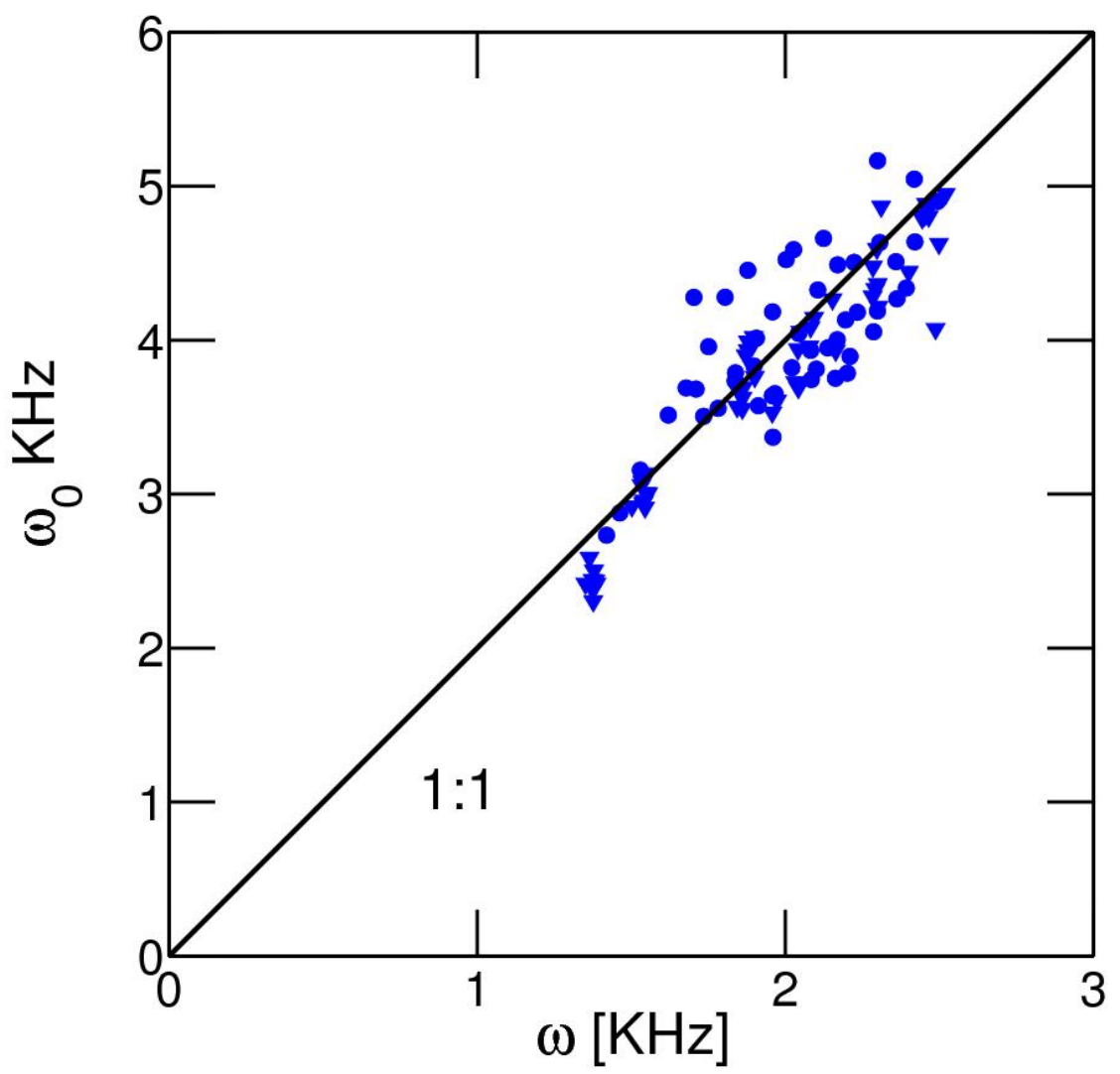

Figure 8.12 Calculated frequency $\omega=\omega_{0} \sqrt{1-\zeta^{2}}$ in function of the free oscillation frequency $\omega_{0}$. Here $\omega_{0} \sqrt{1-\zeta^{2}}=0.4$. The dispersed phase is a mixture of ethanol and water in different proportions are not distinguished here. Two different methods are tested to vary the frequency: first the change of the droplet volume $(\bullet)$, and the change of the droplet speed $(\boldsymbol{\nabla})$. The change in droplet speed has no influence on the frequency, one can notice the group of triangles that share a common surface tension.

The ratio of the oscillation period and the characteristic time of relaxation $\hat{\tau}=\tau_{1} / \tau_{2}$ differentiates two regimes. First an inertial regime in which $\hat{\tau}<1$ and the frequency of oscillations are equal to the frequency of free oscillations $\omega_{0}$. Second, a viscous regime $\hat{\tau}>1$ for which the oscillation frequency $\omega$ differs from the free oscillation frequency $\omega_{0}[146]$. In this second case, a prefactor in the frequency depending on the characteristic time is added 
and the frequency becomes:

$$
\omega=\sqrt{\frac{\gamma}{A H \rho_{\text {in }}}\left(1-\zeta^{2}\right)}=\omega_{0} \sqrt{1-\frac{1}{\omega_{0}^{2} \tau_{1}^{2}}}
$$

From the value of $\zeta^{2}$ we can determine the mode of decay of the oscillations.

- $\zeta^{2}=0$, the regime is periodic without energy dissipation.

- $0<\zeta^{2}<1$, the regime is underdamped with a frequency defined by equation 8.16

- $\zeta^{2}>1$, the regime is overdamped, no oscillations are visible.

In the case of free droplet oscillations the ratio is equal to the Ohnesorge number $\hat{\tau}=O h$. In our ensemble of experiments we calculate the value $O h \sim 10^{-2}$. The corresponding ratio in our experimental conditions differ due to $\tau_{1} \neq \tau_{\text {visc }}$ presented above on figure 8.10 . As a consequence, in our experimental condition we consider the value of $\hat{\tau} \sim 0.2-0.9$. This indicates that our system is situated close to the limit between the viscous regime and the inertial regime.

We show in figure 8.12 the frequencies $\omega$ obtained from the experiments, in comparison to the free oscillation frequency $\omega_{0}$. The droplet surface tension, and droplet fluid densities are presented in the table 8.1 . In this figure we see a good agreement for $\omega \sim 0.4 \omega_{0}$, which corresponds to a values of $\zeta^{2} \sim 0.85$. This values are also in quantitative agreement with the ratio $1 / \tau_{1}^{2} \omega_{0}^{2}$ and corresponds to an underdamped regime close to the critical values $\left(\zeta^{2}=1\right)$. This value correlates well to the observation of a small amount of period, in general 2 or 3 , before the droplet becomes definitively relaxed.

We remark in this study that the dissipation time $\tau_{1}$ is one order of magnitude smaller than the typical period of the droplet oscillation $\tau_{2}$. From the comparison of these two quantities we should not be able to see oscillations. Possible additional effects, arising from the droplet advection in the external flow profile or the droplet confinement, that are not taken into account here, might have an influence the droplet oscillations.

\subsubsection{Oscillations in presence of surfactant}

In the first part of this thesis (chapter 4), we have seen that one consequence of the tangential shear on a laden-interface, results in the reorganization of the adsorbed monolayer, and the emergence of Marangoni forces[112, 116, 147]. In the presence of adsorbed layer, the 
development of interfacial viscoelasticity introduces a lag in the typical relaxation time of the interface[61].

We perform the analysis of the droplet deformation and relaxation kinetics in one chamber in presence of an adsorbed monolayer of surfactant. The aim of this part is to study the possible effect of an additional term coming from interfacial viscoelasticity, that would contribute to changes in the oscillations.

In this part we test the model of a damped oscillator described by the equation 8.14 , in presence of surfactant, where surfactant molecules are dissolved in oil and diffuse to the interface. One series of experiments is performed with macromolecules PEG-PFPE in various concentrations, the second series is performed with Krytox-NH4 molecules. The size of the droplet is kept constant for all the series, but the speed of the droplet is actively modified. The droplet interface is created free from surfactant and spontaneous adsorption occurs along the delay line, as described in chapter 7. All the experiments are performed at a fixed point in the channel. The age of the interface at the measurement point, is then reduced as the droplet speed is increased. Due to the diffusion of the surfactant to the interface the surface tension of the droplet decreases with time. We measure different surfactant concentrations in order to determine if a threshold in the interface coverage can be found for which the presence of Marangoni effect would modify the oscillations. A different experiment follows a droplet along the delay line, in the same way as in chapter 7 in order to see if the dynamics of surfactant adsorption are measurable. The obtained oscillation frequencies are presented on figure 8.13 (a). For reference the experiment performed with a water/ethanol mixture are displayed in gray.

First, the overall oscillations frequencies for systems with and without surfactant follow the same scaling law. From this we can conclude that the Marangoni effects present at the interface during the droplet deformation, have too limited influence to be detected. A large deviation is visible for frequencies at high surfactant concentrations. From the equation 8.16 we know that the droplet oscillation period $1 / \tau_{2}$ is inversely proportional to the surface tension. In addition from the geometry of the deformation chamber we defines a residence time of the droplet $\tau_{\text {res }}$ based on the droplet speed inside the channel $U_{\text {defo }}$ and half the length of the chamber $L_{\mathrm{c}}$, which is where the droplet relaxation is observed $L_{\mathrm{c}}=250 \mu \mathrm{m}$.

$$
\tau_{\text {res }}=\frac{L_{\mathrm{c}}}{U_{\text {defo }}}
$$

For a typical droplet speed $U_{\text {defo }}=400 \mathrm{~mm} \cdot \mathrm{s}^{-1}$ (from figure 8.11). The residence time in the 


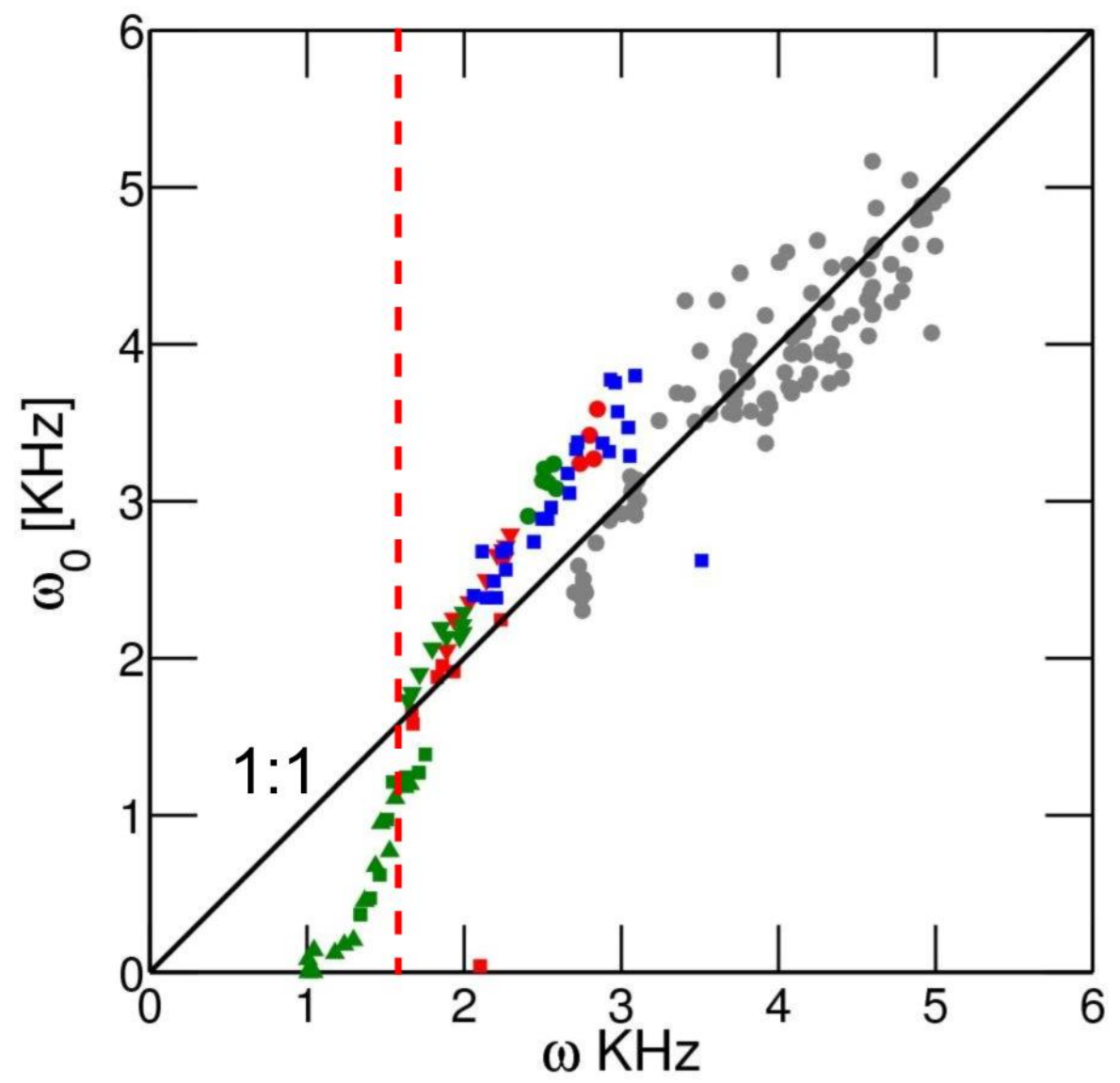

Figure 8.13 Frequency $\omega$ extracted from the oscillation pattern of the droplet inside the channel. Compared to the free oscillation frequency $\omega_{0}$. The data obtained without surfactant is represented by $(\bullet)$. The colored points correspond to the experiments performed with two different molecules PEG-PFPE and Krytox-NH4. The concentrations used are for PEG-PFPE: $C_{\mathrm{PEG}-\mathrm{PFPE}}=1.3 \times 10^{-2} \mathrm{~mol} \cdot \mathrm{m}^{-3},(\bullet) ; C_{\mathrm{PEG}-\mathrm{PFPE}}=$ $2.6 \times 10^{-1} \mathrm{~mol} \cdot \mathrm{m}^{-3},(\nabla) ; C_{\text {PEG-PFPE }}=1.04 \mathrm{~mol} \cdot \mathrm{m}^{-3},(\square) ; C_{\text {PEG-PFPE }}=1.3 \mathrm{~mol} \cdot \mathrm{m}^{-3}$, (^). For Krytox-NH4: $\quad C_{\text {Krytox-NH4 }}=2.6 \times 10^{-1} \mathrm{~mol} \cdot \mathrm{m}^{-3}(\bullet) ; \quad C_{\text {Krytox-NH4 }}=$ $1.3 \mathrm{~mol} \cdot \mathrm{m}^{-3}(\mathbf{\nabla}) ; C_{\mathrm{Krytox}-\mathrm{NH} 4}=6.5 \mathrm{~mol} \cdot \mathrm{m}^{-3}(\mathbf{\square})$. The oscillation of an additional concentration $C_{\mathrm{Krytox}-\mathrm{NH} 4}=2.6 \mathrm{~mol} \cdot \mathrm{m}^{-3}(\boldsymbol{\square})$ have been extracted not only in the last expansion, but all along the delay line. The frequencies on the left of the red dashed line could not be resolved efficiently due to the limited residence time $\tau_{\text {res }}$ of the droplet in the chamber.

channel is $\tau_{\text {res }}=6 \times 10^{-4} \mathrm{~s}$. These value indicates that the period of one oscillation becomes larger than the residence time for values of the frequency below $\omega=1.6 \mathrm{kHz}$. This value is consistent with the shift observed in the determination of the droplet oscillation frequency, visible in figure 8.13 , left off the red dashed line.

We can from this plot, measure the dynamic surface tension of the droplet. It is possible 
to follow the progressive changes in the oscillation frequencies as the droplet interface gets older. It is demonstrated by two methods. One is keeping the position of the measurement fixed, and changing the speed (green and red symbols). The other one is measuring the droplet oscillations as the droplet travels along the delay line (blue symbols). This technique has the advantage to depend only on one parameter other than the surface tension, namely the droplet volume, which is easily measurable in microfluidics.

In summary, we observe inertial relaxation of the droplet after entering the relaxation chamber. The relaxation features a oscillatory behavior, clear enough to be extracted. The oscillation could be characterized in a satisfying fashion with a simple model of damped harmonic oscillator. In presence of the perfluorosurfactant characterized in the chapter 6 , no significant effects due to the presence of Marangoni force at the interface was measured. Therefore the direct extraction of the dynamics of surface tension by the measure of the frequency of the droplet oscillations is possible. The measurement is however limited for low surface tension where the frequency of oscillation become too long to be extracted in our geometry.

\subsection{Conclusion}

In this chapter, we have first evidenced significant discrepancies between a two dimensional numerical simulation and the droplet deformation experiment performed in three dimensional channels, questioning the validity of the Darcy description in this case. We interpreted these deviations, particularly in a non confined cases, by our lack of means to detect the deformation of the droplet interface in the z-direction i.e. out-of-plane deformations.

In the second part, we tested the validity of predictions made for the deformation of three dimensional spheroids droplet in an elongational flow. The agreement of the model with our experimental data was found satisfying in the limit of measurable droplet deformation. However the deviation of the droplet shape from a spheroid and the reduced part of our geometry where a elongational flow make the result imprecise. In a third part we studied the appearance of an inertial regime where the droplet deformation is characterized by oscillations measurable for $R e>10$. The frequency of the oscillations could be compared to a model of damped oscillations derived from the Navier-Stokes equations. The measured frequencies where in good agreement with this model. However the dissipation processes is influenced by the flow profile around the droplet (advection) and the presence of the channel wall (confinement).

The same experimental conditions where also tested with a partial to complete adsorbed 
surfactant layer on the droplet interface. The viscoelastic contribution present due to the compression/decompression pattern of the interfacial deformation, was not found to have a noticeable influence on the droplet deformation. We can conclude that these processes are either not able to develop due to the short time scales of the experiments $\sim \mathrm{ms}$, or they are too limited to compete with the viscous stresses at the interface coming from the surrounding fluids. In addition the measurement of the frequency allows us to follow dynamically the surface tension along for $\gamma>5 \mathrm{mN} \cdot \mathrm{m}^{-1}$. Below the residence time of the droplet in our channel becomes too small for an efficient detection of the oscillation period.

In conclusion this chapter confirms that the dynamic processes of droplet deformation, and relaxation at a planar sudden expansion are suitable for the study of dynamic effects related to simple interfaces. However this study shows the difficulty of describing the flow in geometries which are to large to be compared to a two dimensional model, but where interactions with the channel walls are not negligible. In the particular case of the droplet oscillations the inertial part of the relaxation, could be well described by the model of an interface free relaxation. However the viscous relaxation of the droplet was altered by the presence of the wall and the flow profile around the droplet.

We note that the description of the interfacial properties through its maximum deformation $\delta_{\max }$, as in chapter 7 allow an access to the droplet interface properties that are free of constrains on the droplet shape. Therefore this analysis is applicable in a more general context than the models discussed in this chapter. Naturally the next step would be to test this technique in the context of more complex interfaces, where viscoelastic effects are likely to appear. For instance in the case of an adsorbed layer of colloids, or the case of a polymer network present at the interface. The following chapter will be dedicated to the preliminary study of droplet deformations applied to the monitoring in-situ of interfacial polymerization, where the contribution of viscoelasticity of the layer will be dominant. 



\section{Monitoring polymerization at the droplet interface using microfluidics}

\subsection{Introduction}

The development of the droplets as a microreactor[39], and the implementation of microfluidics fast screening and sorting techniques[74], has allowed for a whole branch of microfluidics to focus towards the development of biomedical applications. The fast and easy control of droplet production allows for the production of large single droplet assays for drug screening[148]. One step further consists in the domains of drug targeted delivery[149]. In this paradigm, the droplet is used as an original type of drug carrier. Droplet-based capsules or liposphere are produced on-chip, where the conditions of production allow the precise control of the droplet composition. This application also carries the idea that the recognition of the site of delivery is programmed in the droplet with, for example with the help of specific markers at the interface. An example of such interface is presented in figure 9.1. Fundamentally, the droplet is now seen as a possibility to design minimum biomimetic systems [150], where the droplet environment is tuned towards the reproduction of the basic functions of a living system.

In all of these applications the interface plays an decisive role as a place of exchange and 


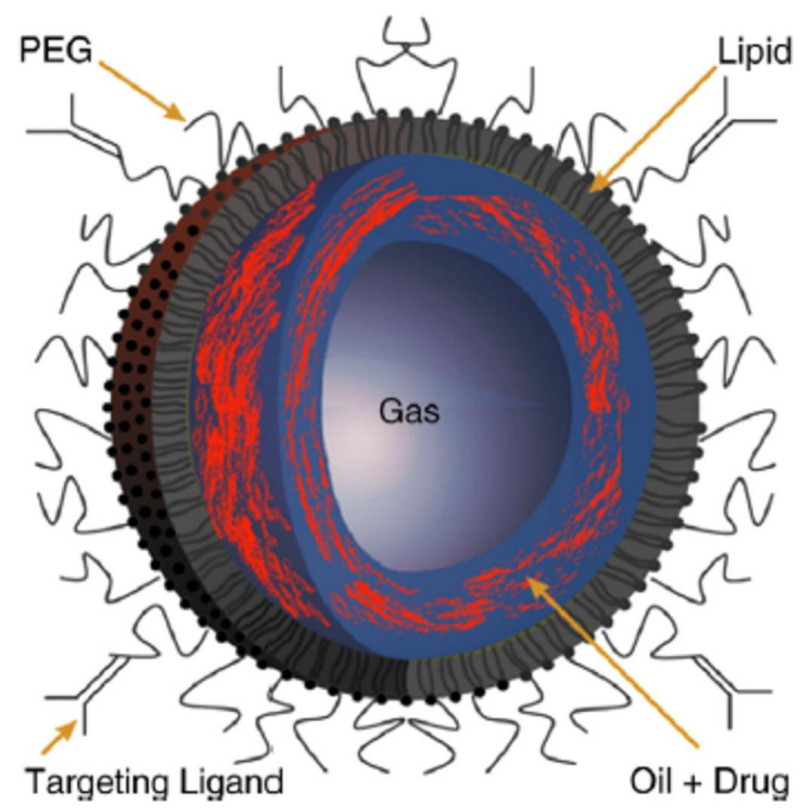

Figure 9.1 Example of a complex interface designed for the purpose of a target delivery. Here the stability of the ensemble is ensured by a lipid membrane. A targeting ligand is also present at the interface for recognition of the site. Reprint from[149]

interaction with the external environment and also as protective barrier confining the droplet volume. The aforementioned applications require that the interfaces can be adapted to the need of the applications. Standard droplet production which uses a surfactant adsorbed layer for the droplet interface stabilization are in certain cases permeable to chemicals[71]. Therefore the suitability of droplets to these new applications requires the fabrication of micrometric compartments with controlled complex interfaces that can be functionalized in order to fit the function of the droplet and provide mechanical stability, biocompatibility, and ensure the controlled permeability of the interface. The production of microcapsules, based on polymer networks or lipid bilayers in microfluidic has already been demonstrated[151, 152].

The characterization of the production of such capsules is also necessary to predict the future properties of the compartment. The microfluidic environment constitutes here as well a convenient platform to monitor processes such as the growth of a polymer network at the interface, or the formation of the colloidal capsule.

This chapter presents the application of the microfluidics techniques developed in chapter 7 to the monitoring of a polymerization reaction occurring at water/oil interface. In this analysis, although preliminary, we show that it is possible to follow the kinetics the formation of a 
polymer network at the droplet interface, by the analysis of the droplet maximum deformation. In addition, we will show that with the formation of the polymer network at the interface, the viscoelastic effects at the interface are progressively overcoming the surface tension effects. Therefore the idea developed in the previous chapters that the interface can be described by the surface tension finds its limits in the study of more complex interfaces.

The chapter is divided as follows: part one describes our experimental systems and the polymerization reaction that is occurring at the droplet interface, and part two the general shape of the reaction kinetics that can be obtained by measuring the deformation of the droplet is presented. We will comment on the general changes that can be seen in the droplet deformation profile as it passes through the deformation chamber. In the last part, we discuss the dynamics of the droplet maximum deformation $\delta_{\max }$ along the channel. We study this dynamics for different reagent concentrations, and in presence of an additional surfactant molecules in the oil phase. We acknowledge the collaboration of Dr. Ingmar Polenz for the following study.

\subsection{Experimental section}

\subsubsection{Geometry}

We use the same microfluidics planar expansion design than described in the previous chapters to study the deformation of the droplet interface where a polymerization reaction between a diisocyanate (contained in the oil phase) and a polyethyleneamine (contained in the water phase) occurs. We extract the droplet maximum deformation $\delta_{\max }$ from the droplet deformation profile in the deformation chamber.

The main changes in the design structure are localized in the production area. The interfacial polyurea formation starts occurring quasi-instantaneously after contact of the two phases containing the reagents. The immediate rigidification of the shell would lead to a clogging of the device in the droplet production region. In order to overcome these difficulties, an additional solvent flow stream is inserted, containing no reagent in order to delay the reaction from the production site. This geometry allows us to control the triggering of the reaction. See figure 9.2. The PDMS channel has a height $H=93 \mu \mathrm{m}$ and is shorter (only thirty expansions). This length is the best compromise between the speed of the reaction and the pressure drop that arise from flowing at high speed a viscous oil in confined environment. The typical flow rates used in this study are: $Q_{\text {oil }}=40.8 \mu \mathrm{L} \cdot \min ^{-1}$ for the oil phase; $Q_{\mathrm{H} 2 \mathrm{O}}=1.6 \mu \mathrm{L} \cdot \mathrm{min}^{-1}$ 


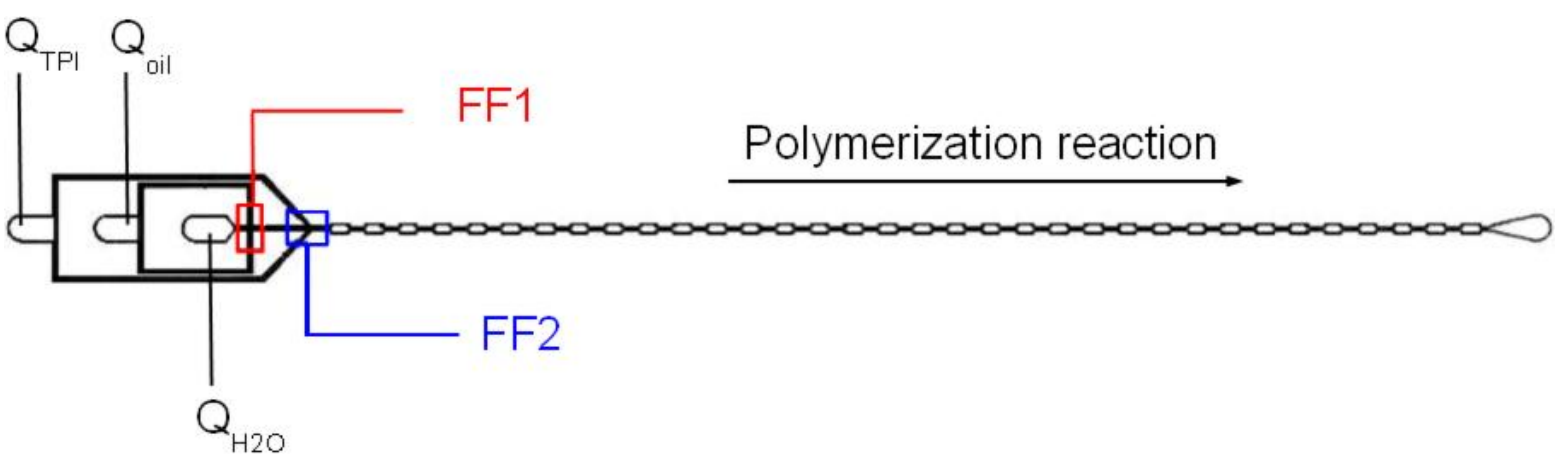

Figure 9.2 Microfluidic design used for monitoring the interfacial polymerization kinetics. The droplet of water + HMDA are created at the first flow focusing geometry (FF1 red square). The reaction is then triggered by the injection of the oil + TDI mixture at the second flow focusing region (FF2 blue square). The height of the channels is $H=93 \mu \mathrm{m}$. The deformation analysis is made in the expansions following FF2. The typical flow rate used in this study are: $Q_{\mathrm{oil}}=40.8 \mu \mathrm{L} \cdot \min ^{-1}$ for the oil phase; $Q_{\mathrm{H} 2 \mathrm{O}}=1.6 \mu \mathrm{L} \cdot \min ^{-1}$ for the water phase, and $Q_{\mathrm{TDI}}=17.5 \mu \mathrm{L} \cdot \mathrm{min}^{-1}$ for the additional oil phase containing the diisoocynate.

for the water phase, and $Q_{\mathrm{TDI}}=17.5 \mu \mathrm{L} \cdot \mathrm{min}^{-1}$ for the additional oil phase containing the isocynate

\subsubsection{Polymerization reaction and chemicals}

For our measurements we used a polyaddition reaction to form polyurea network at the interface. The reaction occurs between a solution of 1,6-hexamethylenediamine (HMDA) in the droplet water phase, and a 2,4-toluene diisocyanate (TDI) in the oil phase. The reaction is depicted in Figure 9.3. The main advantage of this reaction type is, besides the rapidity and robustness, the absence of any side products formation that would influence the reaction course. Such reaction are known to occur at polycondensation reactions such polyamide and polysiloxane interfacial polymerization[152]. The reaction can be then represented as:

$$
A+B \rightarrow C
$$

It is to be noted that the polyurea ( $\mathrm{C}$ compound in this case) chains that are produced have free reactive sites and reactions of types $(\mathrm{A}+\mathrm{C})$ and $(\mathrm{B}+\mathrm{C})$ are possible. The overall cross linking of the interface occurs via $(\mathrm{C}+\mathrm{C})$ reactions. The isocyanate, 2,4-Toluene diisocyanate (TDI) as well as the amines 1,6-Hexamethylenediamine (HMDA), were purchased in the highest available purity grade from Sigma Aldrich and used without further purification. The surfac- 


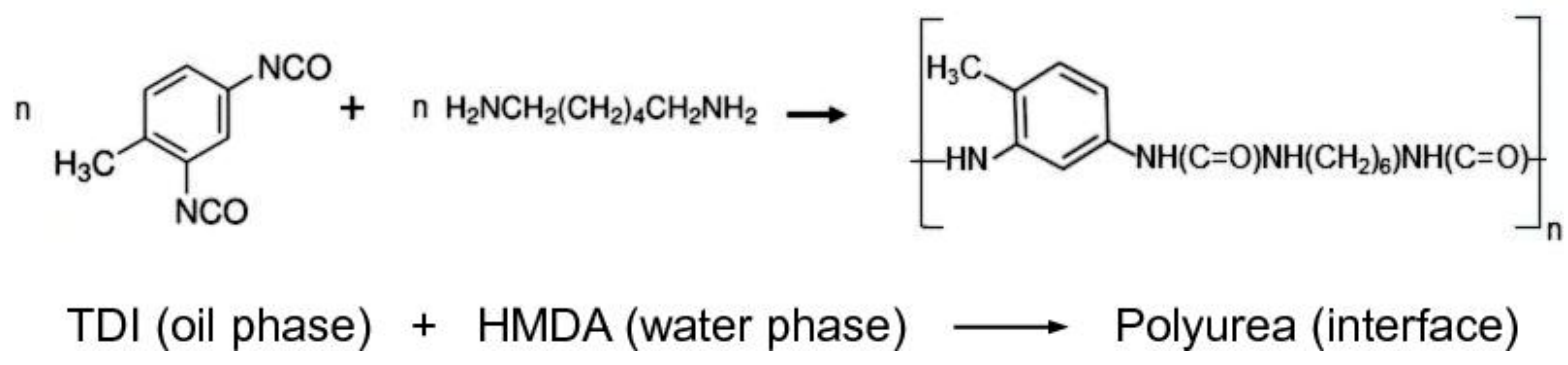

Figure 9.3 Polymerization reaction between TDI and HMDA occurring at the droplet interface

tant Abil EM 90 from Evonik Industries was also used as received. We used KMC oil 113 (a mixture of 2,6- and 1,5-diisopropylnaphthalene) as the oil is filtered on a alumina bead column before use. The viscosity of the oil was measured using a cone-plane rheometer. A stable value of $\eta_{\mathrm{KMC}}=8 \mathrm{mPa} \cdot \mathrm{s}$. The oil density is $d_{\mathrm{KMC}}=$ We determine the oil $/$ millipore water interfacial tension using Wilhelmy plate methods, described in chapter 2 . The interfacial tension was measured to $\gamma=35 \mathrm{mN} \cdot \mathrm{m}^{-1} \pm 2 \mathrm{mN} \cdot \mathrm{m}^{-1}$.

\subsection{Dynamics of interfacial polymerization monitored on- chip}

\subsubsection{Profile of deformation of the interface}

The droplet deformation is followed on two different time scales. First the profile of the droplet is recorded in one chamber. Second, the droplet maximum deformation $\delta_{\max }$ is followed and along the whole delay line.

First we focus on the short time scale analysis, i.e. the comparison of the droplet deformation profile within one microfluidic expansion, at different reaction stages of the polymerization which allows us to conclude on the mechanical properties of the interface. Figure 9.4 shows the droplet deformation profile as it enters the planar sudden expansion, the interface undergoes a well-known transversal deformation and relaxation. The comparison of the droplet deformation profiles in the figure 9.4 (c) allow us to compare these profile for a non cross-linked interface (black points) at the beginning of the channel and an interface where the reaction has taken place (gray points) at the end of the delay line. We notice that in the last case the droplet maximum deformation $\delta_{\max }$ although visible remains negative, which corresponds 

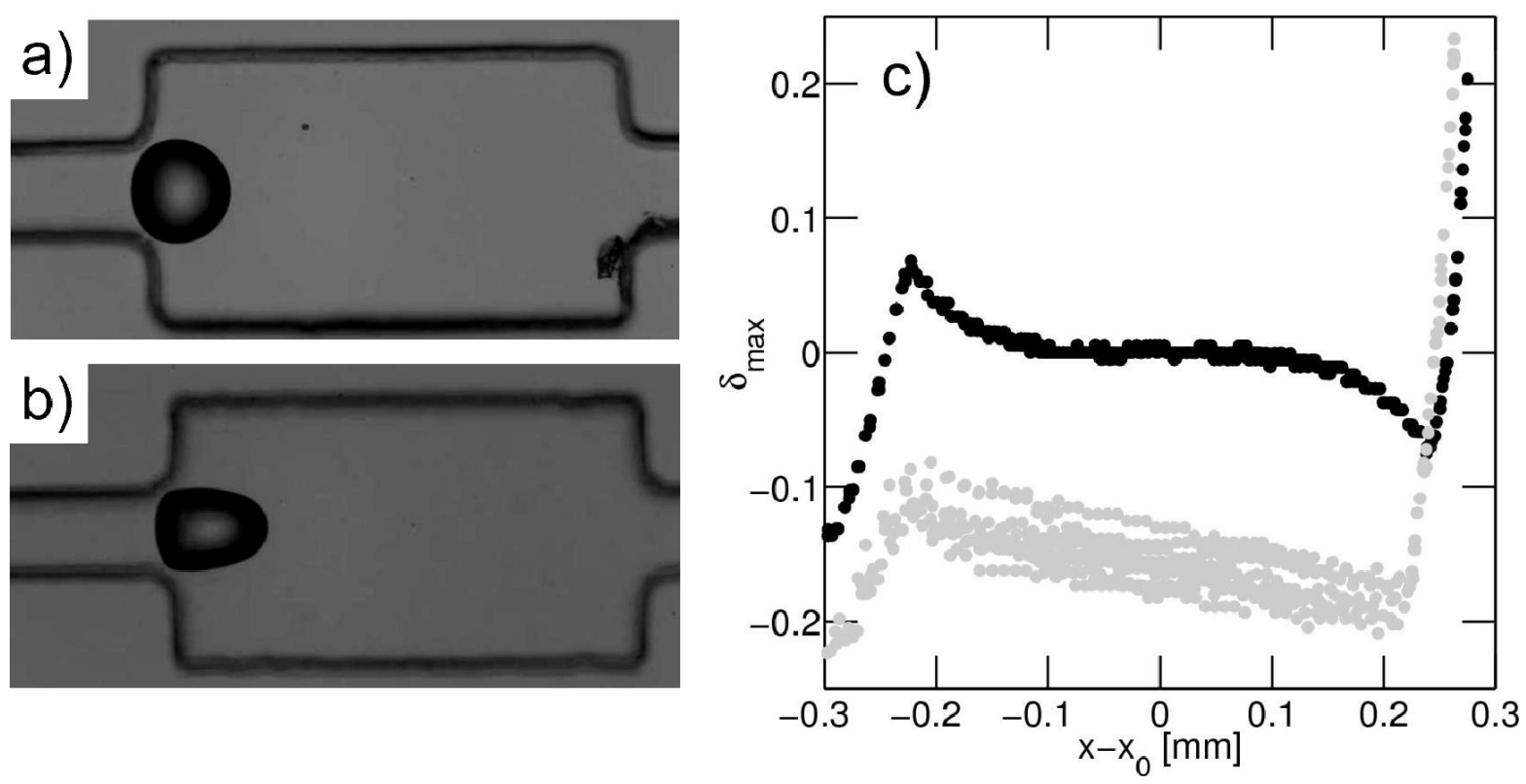

Figure 9.4 Micrograph of a droplet at its position of maximum deformation (a) after production, when the reaction has not been triggered and (b) at the end of the channel for high HMDA concentration $\left(C_{\mathrm{HMDA}}=43 \mathrm{~mol} \cdot \mathrm{m}^{-3}\right.$ and $C_{\mathrm{TDI}}=128 \mathrm{~mol} \cdot \mathrm{m}^{-3}$. (c) The corresponding deformation profiles when droplet is passing through the channel. The gray point corresponds to an advanced stage of the polymerization, the values are negative as the droplet remains prolate in the expansion.

to a prolate shape.

In that case we can deduce that the hydrodynamic viscous stress at the interface, is to small to provoke the transversal deformation of to the flow. The droplet interfacial energy is then increasing with the presence of the polymer network, and a larger viscous forces at the interface are needed for the deformation.

In addition, we note that the droplet relaxation in the chamber does not converge to a sphere while passing thought the deformation chamber. These behaviors are resolutely different from the non cross-linked interface, and lead us to the conclusion that in this case, the mechanical properties of the interface can not be described as done previously, i.e. taking the surface tension as the unique parameter describing the interface. The polymer network that is formed at the interface is more complex than the adsorbed surfactant monolayer described in the previous chapter of this work. The important numbers of interactions between the molecules and the thickness of the polymer layer that is formed[153, 154] can not be described in terms of surface tension only. A more rigorous description is required, such as a membrane 
mechanically described in terms of elastic and viscous moduli. In that case the characteristic time of interface relaxation is strongly linked to the interfacial viscosity.

From this observation we are able to determine a point in the kinetics of the reaction where the droplet relaxation in the chamber do not reach a plateau corresponding to an equilibrium deformation anymore. This point allow us to determine in a qualitative way the relative value of the viscous time related to the interfacial viscosity, and the residence time of the droplet in the chamber.

On a larger time scale the dynamics of the droplet maximum deformation $\delta_{\max }$ gives us information on the kinetics of the polymerization reaction present at the interface. In the following part of this study we are interested in the parameter that can be explored to better understand the reaction. We will present the droplet maximum deformation dynamics as a function of both reagent concentrations, and the presence of surfactant.

\subsubsection{Dynamics of the droplet maximum deformation}

\section{Variation of the HMDA concentration}

In order to understand the effect on the kinetics of the reagent concentration, we start by varying the aqueous droplet composition. In figure 9.5 we present the dynamics of the droplet maximum deformation using a fixed concentration of TDI $C_{\mathrm{TDI}}=128 \mathrm{~mol} \cdot \mathrm{m}^{-3}$, and for concentrations of HMDA ranging from $C_{\mathrm{HMDA}}=0 \mathrm{~mol} \cdot \mathrm{m}^{-3}$ to $C_{\mathrm{HMDA}}=45 \mathrm{~mol} \cdot \mathrm{m}^{-3}$.

From figure 9.5 we notice a difference in the dynamics of the droplet maximum $\delta_{\max }$, between the case were one reagent is missing and when HMDA is present in the droplet phase. In absence of HMDA, the droplet deformation, is constant and positive. In this case there are no reactions changing the properties of the interface occurs in the time scale of our measurement. The TDI alone in the oil does not present surface active properties that would influence the dynamics of droplet maximum deformation.

In presence of the HMDA reagent, the dynamics of the maximum deformation starts with a small plateau around a value of $\delta_{\max -\mathrm{i}}=0.05$. This is followed by a decrease until reaching a final negative value $\delta_{\max -\mathrm{f}}$ which is also a plateau for the high concentrations of HMDA, for interface ages around $\sim 1 \mathrm{~s}$.

The presence of the small plateau in the beginning of the dynamics can be explained the times it takes for the HMDA to reach the interface and start the reaction. From the previous study of the surfactant in chapter 7 kinetics we know that the transport of matter to 

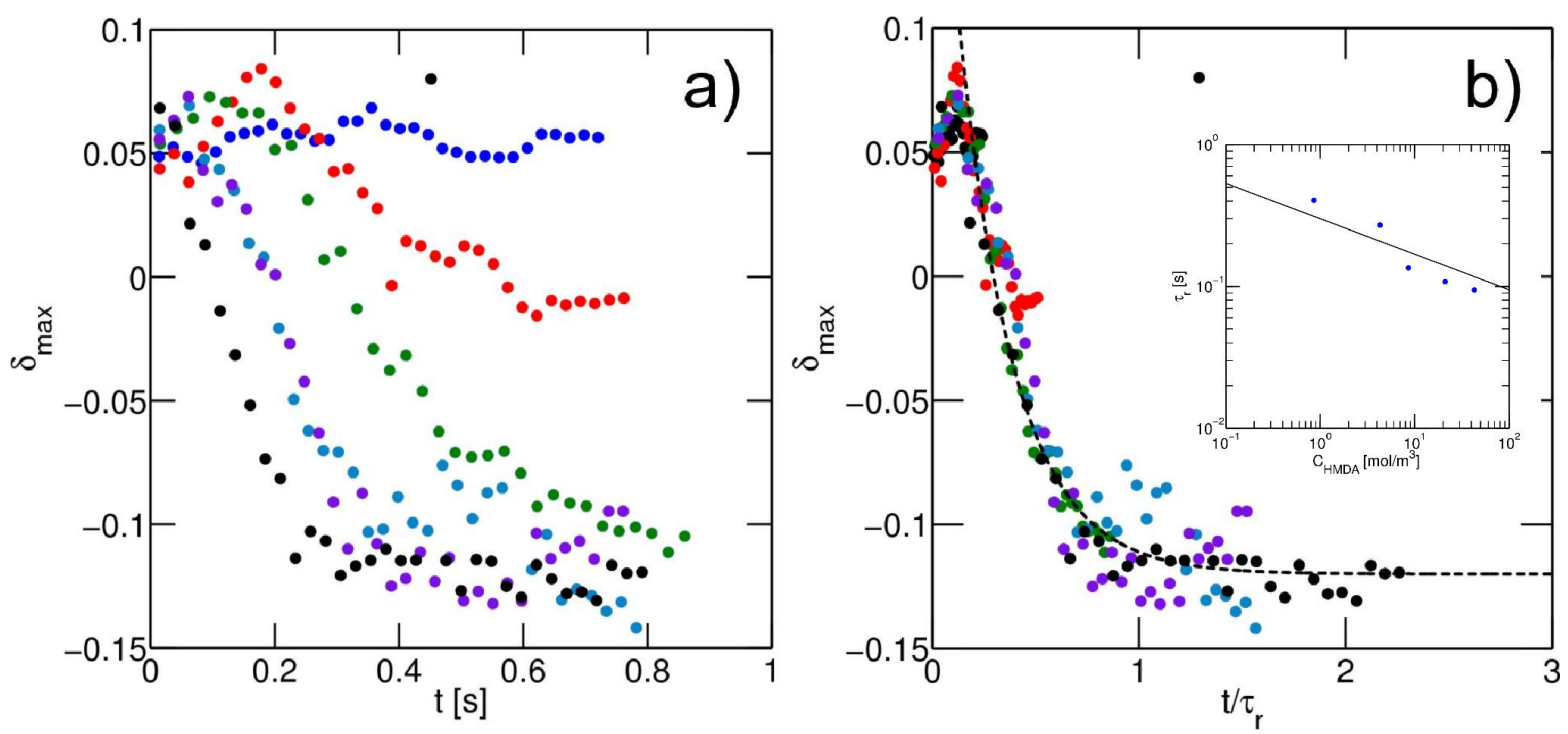

Figure 9.5 (a) Monitoring of the maximum deformation of the droplet entering the expansion for different HMDA concentration: $C_{\mathrm{HMDA}}=0 \mathrm{~mol} \cdot \mathrm{m}^{-3}(\bullet) ; C_{\mathrm{HMDA}}=0.86 \mathrm{~mol} \cdot \mathrm{m}^{-3}(\bullet)$; $C_{\mathrm{HMDA}}=4.3 \mathrm{~mol} \cdot \mathrm{m}^{-3}(\bullet) ; C_{\mathrm{HMDA}}=8.6 \mathrm{~mol} \cdot \mathrm{m}^{-3}(\bullet) ; C_{\mathrm{HMDA}}=21 \mathrm{~mol} \cdot \mathrm{m}^{-3}(\bullet) ;$ $C_{\mathrm{HMDA}}=45 \mathrm{~mol} \cdot \mathrm{m}^{-3}(\bullet)$, for a constant TDI concentration of $C_{\mathrm{TDI}}=128 \mathrm{~mol} \cdot \mathrm{m}^{-3}$. (b) Rescaling of the deformation kinetics in on an exponential decay $\sim \exp ^{\left(-\mathrm{t} / \tau_{\mathrm{r}}\right)}$, resulting in a dependence $\tau_{\mathrm{r}} \sim C_{\mathrm{HMDA}}^{-1 / 4}$ visible in the inset.

the interface is dominated by convective effects. Here we see that the length of the plateau is only slightly affected by the changes in reagent concentrations for concentration under $C_{\mathrm{HMDA}}=21 \mathrm{~mol} \cdot \mathrm{m}^{-3}$, which is consistent with this model. Above this concentration, the plateau is hardly measurable, which indicates that the concentrations are high enough for HMDA to be immediately present at the interface. This could be the sign of the change of the regime of the transport of the HMDA to the interface.

The dynamic of the droplet maximum deformation is visibly dependent on the HMDA concentration. See figure 9.5 (a). The form of the dynamics is well fitted by an exponential decay. We extract the typical time scale of the reaction, $\tau_{\mathrm{r}}$ by fitting the series of points corresponding to one experiment with the following expression.

$$
\delta_{\max }=\left(\delta_{\max -\mathrm{i}}-\delta_{\max -\mathrm{f}}\right)+\exp ^{\left(-\left(\mathrm{t}-\mathrm{t}_{\mathrm{i}}\right) / \tau_{\mathrm{r}}\right)}-\delta_{\max -\mathrm{f}}
$$

Where $\delta_{\max -\mathrm{i}}$ and $\delta_{\max -\mathrm{f}}$ are respectively the average values of the initial and the final plateaus of the droplet maximum deformation and $t_{\mathrm{i}}$ is a time offset that account for the duration of the 
initial plateau. For each of the dynamics we are able to extract a typical time scale $\tau_{\mathrm{r}}$ and then rescale the time axis in to obtain a collapse of the data. This is shown in figure 9.5(b). We find the dependence of the reaction time on the HMDA concentration to be $\tau_{\mathrm{r}} \sim C_{\mathrm{HMDA}}^{-1 / 4}$.

\section{Variation of the TDI concentration}
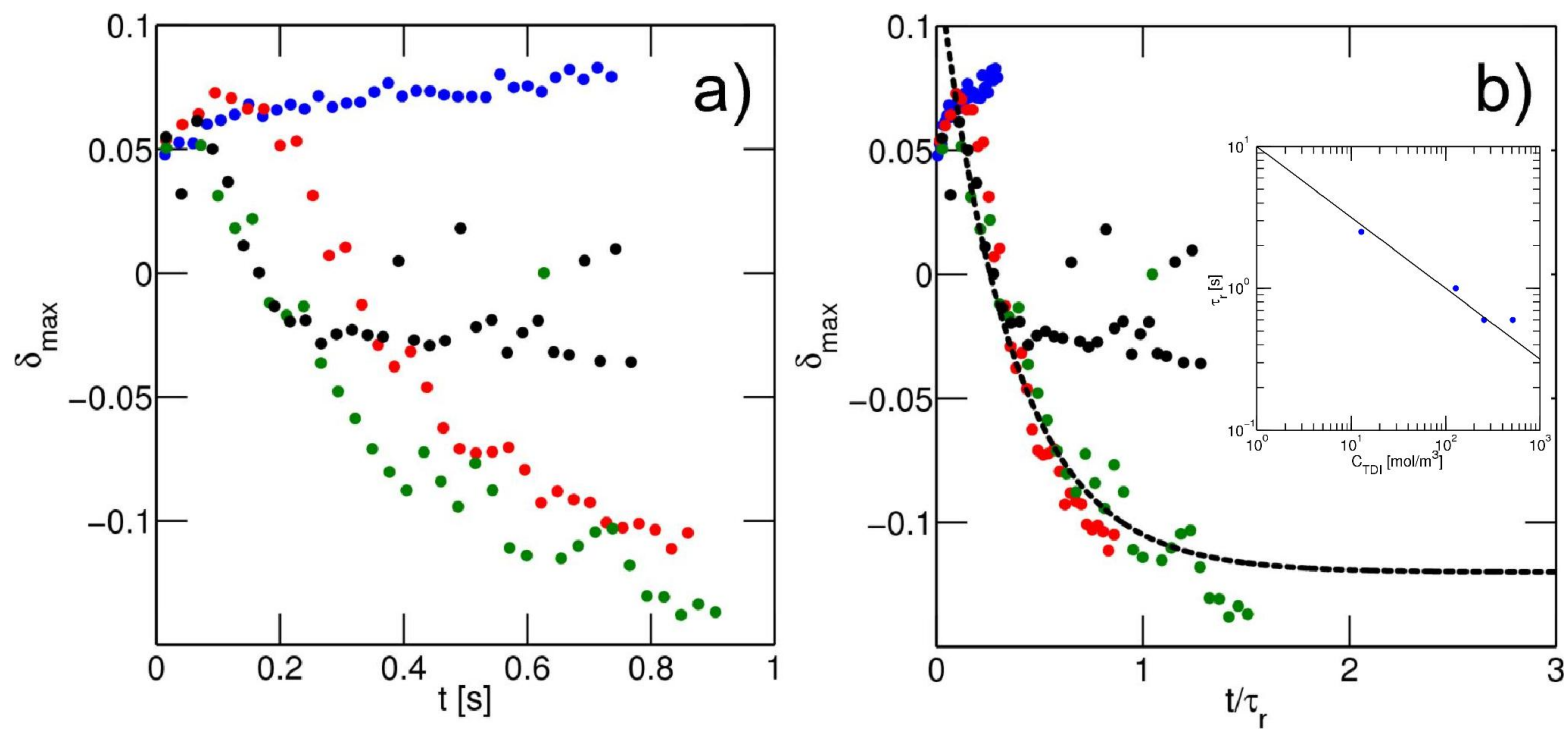

Figure 9.6 Maximum deformation of the droplet entering the expansion for different TDI concentration: $C_{\mathrm{TDI}}=12.8 \mathrm{~mol} \cdot \mathrm{m}^{-3}(\bullet) ; C_{\mathrm{TDI}}=128 \mathrm{~mol} \cdot \mathrm{m}^{-3}(\bullet) ; C_{\mathrm{TDI}}=256 \mathrm{~mol} \cdot \mathrm{m}^{-3}$ $(\bullet) ; C_{\mathrm{TDI}}=513 \mathrm{~mol} \cdot \mathrm{m}^{-3}(\bullet)$ and for a constant $\mathrm{HMDA}$ concentration of $C_{\mathrm{HMDA}}=$ $4.3 \mathrm{~mol} \cdot \mathrm{m}^{-3}$ (b) Rescaling of the deformation kinetics in on an exponential decay $\exp ^{\left(-\mathrm{t} / \tau_{\mathrm{r}}\right)}$, resulting in a dependence $\tau_{\mathrm{r}} \sim C_{\mathrm{TDI}}^{-1 / 2}$.

We perform the same experiment keeping a constant concentration of HMDA to $C_{\mathrm{HMDA}}=$ $4.3 \mathrm{~mol} \cdot \mathrm{m}^{-3}$ and varying the TDI concentration between $C_{\mathrm{TDI}}=12.8 \mathrm{~mol} \cdot \mathrm{m}^{-3}$ to $C_{\mathrm{TDI}}=$ $513 \mathrm{~mol} \cdot \mathrm{m}^{-3}$.

The same general remarks on the general aspect of the dynamics of the maximum deformation can be made. The length of the initial plateau is consistent with the one found in the previous part, and only slightly differs over the large range of TDI concentrations tested.

Here the fit of the same exponential decay as equation 9.2, gives satisfactory results, leading to the same dependence of the reaction time on the TDI concentration to be $\tau_{\mathrm{r}} \sim C_{\mathrm{TDI}}^{-1 / 2}$. We notice however that range of TDI concentrations in which the changes in droplet maximum occurs in our range of is much larger than the HMDA concentration. 


\section{Chapter 9. Monitoring polymerization at the droplet interface using microfluidics}

The particular position of the final plateau for the concentration $C_{\mathrm{TDI}}=513 \mathrm{~mol} \cdot \mathrm{m}^{-3}$ arises from the fact that the droplet interface was very irregular, and thus the deformtation $\delta_{\max }$ could not be extracted properly and therefore these where points not taken into account for the rescaling of the dynamics.

We have seen that the dynamics of the droplet maximum deformation is dependent on the concentration of HMDA and TDI in the two phases. The characteristic time scales that were extracted from the dynamics can not be compared to the assumption a diffusive model, that should exhibit at first order a dependence on the interfacial concentration $C^{-2}$, or a transferlimited model that dependence on the interfacial concentration scales as $C^{-1}$. These two models are based on the fact that the surface tension can linearly be linked to the amount of surfactant at the interface. However the reaction speed seems to be more dependent on the TDI $\left(\sim C_{\mathrm{TDI}}^{1 / 2}\right)$ than concentration than on the HMDA $\left(\sim C_{\mathrm{HMDA}}^{1 / 4}\right)$. This is information is to be linked to the fact that the molar concentration of TDI need to be around ten times larger than the HMDA concentration to monitor a reaction in our time scales. A model of reaction that takes into account such a dependence would be the following. First the interfacial polylmerization in its early stage occurs through the reaction between the amine and the diisocyanate. Later, as the polymer network growth, the polymerization is dominated by the reactions between the diisocyanate molecule and the polyurea molecules at the interface resulting in an asymmetric growth of the polymer network. More experiments are to be performed to confirm this scenario.

In the case of a polymer network, the presence of a general viscoelastic behavior make unlikely a linear dependence of the mechanical properties of the interface to the density of the polymer network. Therefore we are currently limited to a qualitative description of the droplet deformation kinetics that can not be linked to a reaction model at the interface. Here we are lacking of a proper quantification of the deformation on the both the elastic and viscous moduli of the interface to properly address the kinetics of polymer formation at the interface.

\section{Dynamics in the presence of surfactant}

The formation of oil in water capsules is possible using the same microfluidic geometry, by simply reversing the phases and the channel wetting properties. The continuous phases are now aqueous solutions of HMDA and the droplet phase is a solution of oil and TDI. However for wetting reasons, even in presence of a surface treatment of the channel wall, the droplet are highly unstable in the microfluidic confined environments. The addition of surfactant can 

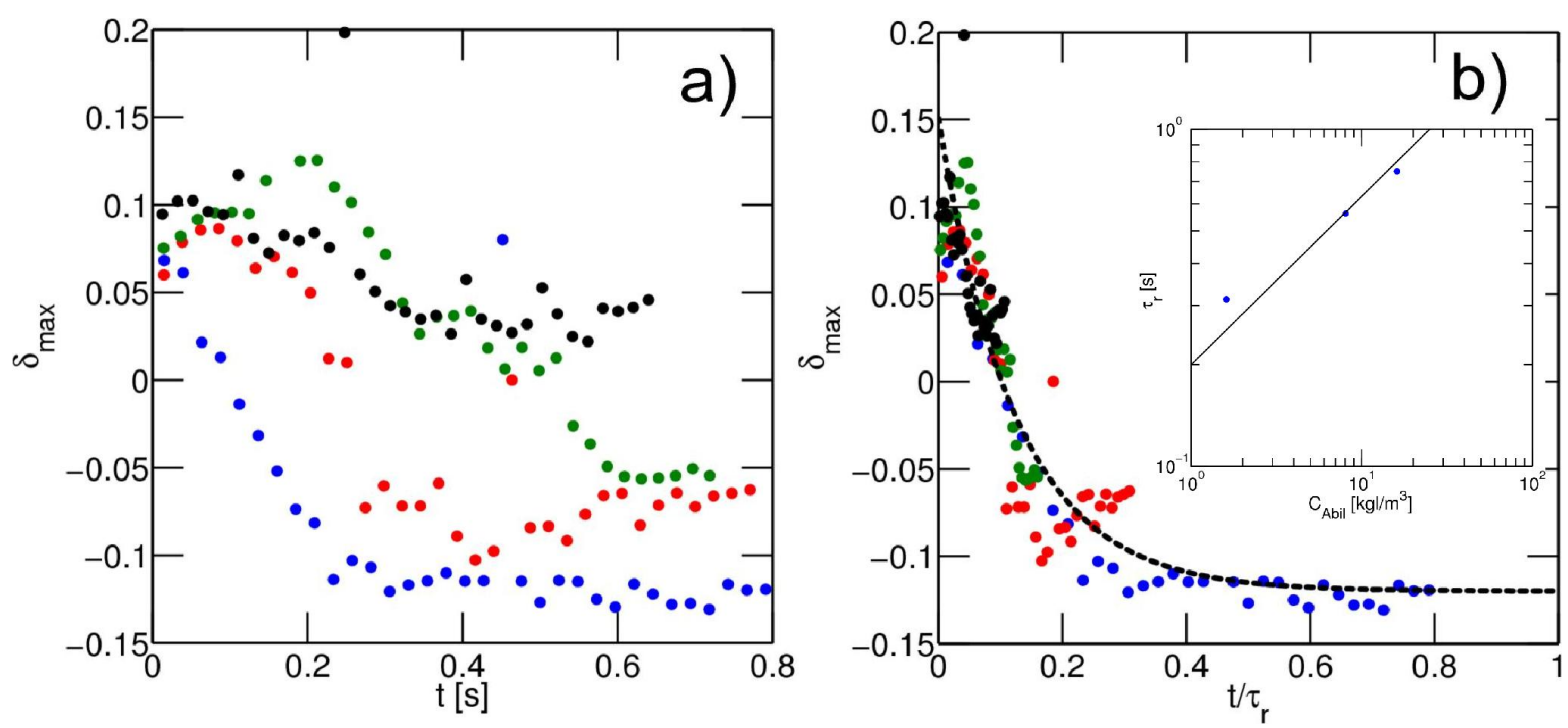

Figure 9.7 Kinetics for a fix concentration of reagents, $C_{\mathrm{HMDA}}=43 \mathrm{~mol} \cdot \mathrm{m}^{-3}$ and $C_{\mathrm{TDI}}=$ $128 \mathrm{~mol} \cdot \mathrm{m}^{-3}$. And different concentrations of the Abil surfactant. $C_{\text {Abil }}=0 \mathrm{~kg} \cdot \mathrm{m}^{-3}$ $(\bullet) ; C_{\mathrm{Abil}}=1.61 \mathrm{~kg} \cdot \mathrm{m}^{-3}(\bullet) ; C_{\mathrm{Abil}}=8.05 \mathrm{~kg} \cdot \mathrm{m}^{-3}(\bullet) ; C_{\mathrm{Abil}}=16.1 \mathrm{~kg} \cdot \mathrm{m}^{-3}(\bullet)$

prevent the wetting effects, but this impacts the dynamics of the reaction, and consequently on the dynamical mechanical properties of the capsule. From the polymerization dynamics presented above we can see that the reaction on chip is taking place in the same time scale as the surfactant transport to the interface, evidenced in the chapter 6. We are interested here to study the possible influence between the two processes occurring at the same time at the interface.

In the present experiment, (figure 9.7) the concentrations of HMDA and TDI are fixed, and the surfactant concentrations varied between $C_{\mathrm{Abil}}=0 \mathrm{~kg} \cdot \mathrm{m}^{-3}$ and $C_{\mathrm{Abil}}=16.1 \mathrm{~kg} \cdot \mathrm{m}^{-3}$. The overall shape of dynamics of the droplet maximum deformation $\delta_{\max }$ is not modified by the presence of surfactant, and the dynamics are still well fitted by an exponential decay.

However, two features differs from the reaction studied above. First, the characteristic time of the reaction (extracted as above) is increasing with the surfactant concentration. Second the dynamics seems to reach plateau in all the cases, but the values of the plateau is different for each surfactants concentrations.

These two features indicate that the adsorption of the surfactant which is occurring has a strong influence on the mechanical properties of the interface. In this case the difference in the values of the final plateau reveals that the equilibrium of the process results in a competition 


\section{Chapter 9. Monitoring polymerization at the droplet interface using microfluidics}

between the surfactant and the polymer. Here, the surfactant promotes the droplet deformation, by the decrease of the surface tension and the growth of the polymer network rigidifies the interface.

Furthermore, the presence of the surfactant at the interface also reduces the sites where the HMDA and the TDI can "meet" to form polyurea. Therefore it is possible that the overall structure of the polymer network is influenced by the presence of the surfactant. Complementary structural analyses are required to address this hypothesis.

\subsection{Conclusion}

In this chapter we have presented an on-going work aimed at proving the possibility to monitor in-situ the formation of a viscoelastic layer on the droplet interface, via interfacial polymerization .

The present studies have shown the limitations of our approach of the droplet deformation for such a complex interface. The deformation of rigid interface can no longer be described by simple models where surface tension dominates. Here the viscoelastic properties arising with the growth of the polymer network at the interface can no longer be neglected in comparison to the surface tension.

However, this study does proves that the dynamics of polymerization at the interfaces can be resolved in microfluidics with time resolution of less than one second. Although the proper models that link the droplet deformation to the viscoelastic properties of the interface are still lacking, this study proposes an original method to monitor the formation of capsule in microfluidic environments.

To improve this work, a quantitative link between the droplet deformation and the mechanical properties of the interface needs to be established. Several models of thin incompressible capsules have been successfully implemented for the description of the deformation in a square microfluidic channel[154], which make us confident in the fact that the mechanical properties of the droplet could be extracted. In addition kinetic models that describe polymerization reactions occurring at the interface between simple molecules as well as for complex polymers have been developed by O'Shaughnessy et al.[155]. The comparison of our dynamics of droplet deformation and these models would allow a characterization of the reaction kinetics taking place at the interface. To complete this study, a structural analysis of the capsule microstructure with SEM, could provide more information on the auto-organization patterns 
resulting from polymerization processes in presence of surfactants.

Finally, we have seen that the shape of the profiles of the droplet relaxation after the deformation in a planar sudden expansion were strongly modified by the growth of the polymer network at the interface. Such differences could not be resolved in the case of a simple surfactant-laden interface. Therefore the characteristic time linked to the interfacial viscosity of the interface can probably be extracted from the study of the droplet relaxation in microfluidics.

We are confident that this principle could be generalized to the characterization of the majority of the complex interfaces such as biological membranes, or colloidal structure at the interface, and the microfluidics could provide original methods for the interfacial rheology of complex interfaces. 



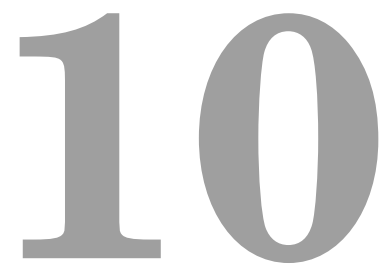

\section{Summary and Outlook}

\subsection{Summary}

We characterized dynamic processes at interfaces, probed through the deformation of a droplet in a microfluidic environment. Three different processes where studied: the adsorption dynamics of surfactant at the interface, the deformation of the droplet interface in confined environment and the kinetics of a polymerization reaction at the interface.

\section{Adsorption of surfactant molecules to the interface}

This work has been first dedicated to the characterization of three perfluorosurfactants with close chemical structures, using the classical pendant drop method. We have studied the dynamics of adsorption of PFPE chains that undergo a dissociation upon adsorption to the interface. We have seen that the concentration of electrolytes in the droplet phase has a strong impact on the surfactant organization at the interface via the screening of the electrostatic interactions between adsorbed molecules at the interface. This changes of organization have an effect on the overall dynamics of adsorption of surfactant, that however remained well described by the scalings of a diffusion-limited model. In most of the cases, the diffusion coefficient of the PFPE chain in the bulk could then be extracted precisely using this measurement. We show that differences in the adsorption kinetics of the PFPE chain can be found for 
two different counterion used to neutralize the PFPE chain. With $\mathrm{H}^{+}$ion the chain dimerization is possible in the bulk phase via the formation of a hydrogen bond. The characteristic time of adsorption were in good agreement with a model taking into account the partition of the surfactant molecules in monomer and dimers in the bulk phase. The implementation of this model allowed us to estimate the energy of the hydrogen bond of the dimer. To our knowledge this is the first measurement of a dimer bonding energy via the pendant drop method.

We designed a dynamic microfluidic tensiometer based on the measurement of the maximum deformation of a droplet $\delta_{\max }$ entering a planar sudden expansion. We formulated an empirical calibration equation that linked the droplet maximum deformation $\delta_{\max }$ its size $R$, and its surface tension $\gamma$. We show that this approach allowed us to perform dynamic tensiometry with a resolution down to the millisecond for a PEG-PFPE molecule of industrial interest. See figure 10.1. Dynamic surface tensions of this systems were consistent with a transferlimited model of adsorption. The comparison to this models allowed determination of the time scale of adsorption - desorption from the interface, which are in a range comparable to the values of measured for other surfactant molecules.

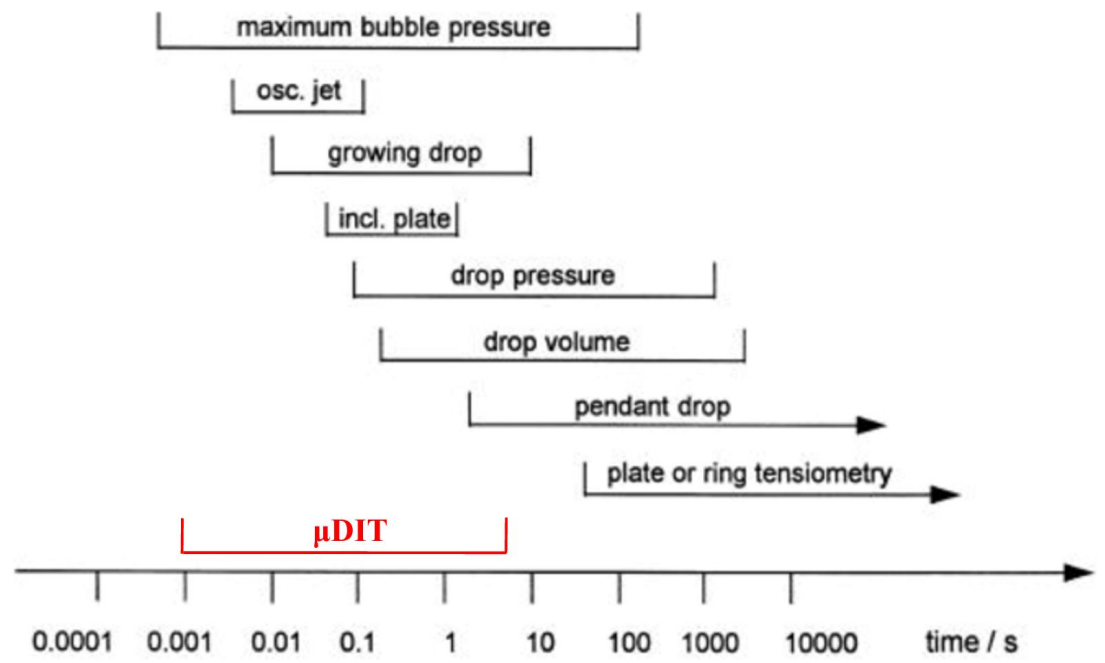

Figure 10.1 Comparison of the time resolution of our on chip tensiometer to other macroscopic techniques.

\section{Interface deformation in microfluidic confinement}

Then, we presented the analysis of the deformation of a droplet in our sudden planar expansion geometry, in order to extract the scalings of the droplet deformation. Experiments were compared to a two dimensional numerical simulation of the droplet contour based on the Darcy 
equation. The overall behavior in both cases were qualitatively following the same trend and two regimes of deformation could be extracted. First, one regime where the droplet deformation depends on its confinement in the channel and on the Capillary number. Second, a regime where the droplet deformation at the expansion is determined by the Capillary number only. The separation between these two regimes was given by the condition $2 R=W$ where the droplet diameter is matching the width of the channel.

However, we found a quantitative discrepancy of the influence of the Capillary number on the deformation. The exponent that is one in the case of the numerical simulation is found to be below one for the experimental case. This discrepancy increases with the the channel height i.e. as the flow conditions deviated from the two dimensional case. We drew the conclusion that our geometry could not be described by the implementation of a two dimensional flow profile based on Darcy law. This conclusion was confirmed with the study of the droplet deformation in an elongational flow profile, with a three dimensional model, that describes the deformation of spheroids in such hydrodynamic conditions. The experimental deformation were in quantitatively satisfying agreement with the three dimensional model in the range of deformation. However the deviation of the droplet shape from a spheroid make this study quantitatively unreliable

A second part of this study was dedicated to the analysis of the droplet deformation in the inertial case featured by the oscillation of the interface during the relaxation in the microfluidic chamber. We show that the oscillations of the droplet in microfluidic conditions can be described by a model derived from the Navier-Stokes equations. In this model, the frequency of oscillations of the interface is inversely proportional to the surface tension and the damping linked to the viscosities of the fluids. The extracted frequencies from the experimental result were in quantitative agreement with this model and could lead to an additional measurement of the surface tension in the microfluidic channel. We show that here also, both confinement and advection of the droplet should be taken into account to fully model the viscous effects at the droplet interface.

\section{Interfacial Polymerization}

Finally, we described an attempt to study the kinetics of a polymerization reaction between a diisocyanate molecule in the oil phase and an amine in the water phase that occurs at the droplet interface. We apply the method developed for the on-chip tensiometry and followed in this case the decrease of the droplet maximum deformation along the delay line linked to 
the stiffening of the interface with the growth of the polymer network. From the profile of the droplet relaxation, we show that the growth of a polymer network at the interface implies the appearance of an interfacial viscoelasticity. Therefore, even if a kinetics of the droplet deformation is extracted, a quantitative relation is still needed to establish a relation between the droplet deformation and the state of the cross-linking at the interface. We could determine the dependence of the deformation kinetics on the reagent concentration, and on the presence of surfactant at the interface. The kinetics are found to be strongly dependent on the concentration of diisocyanate. In the presence of surfactant, the kinetics is slowed down, suggesting a competition between the surfactant adsorption at the interface and the chemical reaction for reaction site at the interface. More experiment are currently performed in order to establish a link between the mechanical properties of the interface, the deformation and the density of the polymer network at the interface.

\subsection{Outlook}

The development of complex interfaces in microfluidics is pushed by the development of methods for compartmentalization and biochemical application that use the presence of polymer networks or lipid layer at interfaces. In addition, alternative methods of emulsion stabilization have been developed using colloidal particles (Pickering emulsions) or the adsorption of large polymer molecules [156]. For complex interfaces, the next step for the microfluidic characterization seems to be the characterizations of interfacial rheological properties originating from the complex interplay between these adsorbed material on the interface.

We have seen that in our geometry and emulsification system, the presence of an adsorbed surfactant layer do not change fundamentally the mechanisms of the interface deformation. Therefore, we concluded that our time scale of deformation were too short to see any influence coming from the reorganization of the adsorbed surfactant layer originating from the Marangoni forces, or the adsorption of surfactant during the deformation. In the contrary the last chapter of this thesis have revealed the non negligible effects of the polymer network formation on the viscoelastic properties of our interface.

Therefore the access to the rheological properties of the interfaces is linked to the time scale of the applied stress on the interface and depends on the nature of the adsorbed layer. Microfluidics is a tool that provides a large range of geometries and flow profiles to fine tune the flow at interface. Here are two examples of experiments that could be implemented in 
microfluidics to give further insight in the mechanical properties of complex interfaces.

First, the hydrodynamic forcing of the interfaces could be easily modulated to apply a periodic stress on the interface with fine tuned frequencies, and amplitudes. Then techniques known as oscillating droplet[61] could be implemented on the micrometer scale in order to extract the viscous and elastic moduli of the interface in conditions of two dimensional dilational rheology.

In addition, Marangoni effects, when developed on a sufficiently long time scale have also a non negligible influence in two phase flows in the presence of surfactant. A good example is the flow in Plateau borders, with a typical size of the order of micrometer, that occurs during the drainage of an emulsion[157]. Here the implementation of a concentric two phase flow in microchannels with the appropriate cross-section, and longer characteristic time scales could serve as a model environment for this type of flow in conditions of elongational rheology.

In summary microfluidics has a true potential to study the dynamics of soft-interfaces. We hope that our approach will be pursued in the future to extract quantitative information on the dynamics of out-of-equilibrium soft interfaces. 



\section{A \\ Measurement of PDMS channel deformation under flow conditions.}

In this appendix we present the study of the PDMS channel deformation linked to the pressure drop that occurs in flow conditions. Although of common use in microfluidics engineering techniques, the PDMS presents a major constraint which is its deformability at high pressure. In this part we show two methods that characterize this deformation under flow, in our microfluidics geometries. In a first part we use the change in droplet projected area to demonstrate the change in the confinement resulting from the pressure drop along the channel. In the second part we compare the wall deformation directly from the inside of the channel using confocal microscopy in presence and absence of flow. We would like to acknowledge the contribution of Dr. Anupam Sengupta for the confocal microscopy measurements.

\section{A.1 Droplet area change}

It is possible to follow the changes in channel deformation along the delay line by measuring the projected area of a confined droplet. We have seen in chapters 7 and 8 that the deformation $\delta$ of a confined droplet in a sudden planar expansion scales like the projected area $A \delta \sim A^{(2 / 3)}$. Therefore a good control of the droplet size all along our microfluidics chip is necessary. We 
notice that the PDMS techniques do not provide the required stability due to the deformation of the walls under pressure. In this part we show how is it possible to estimate the deformations of the top and bottom walls of the channel by following the changes in droplet area along the channel.

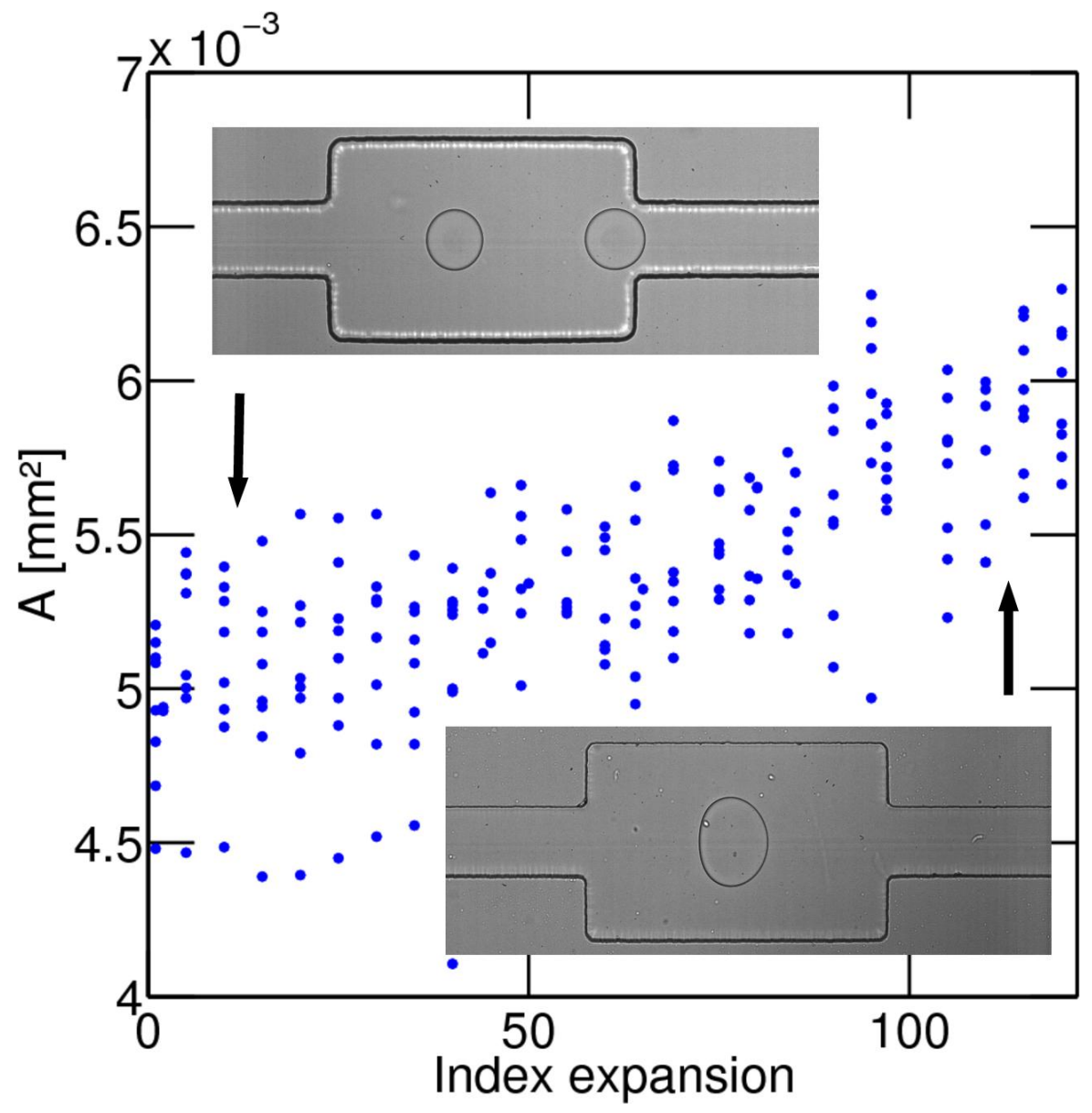

Figure A.1 Increase of the droplet projected area $A$ as a function of its position in the channel delay line (given by the number of the expansion considered). We can see a monotonous trend as the droplet progresses through the channel. The pressure drop along the delay line has a direct consequence on the channel height. As the pressure decreases, the channel becomes thinner, the droplet confinement increases. Inset Micrograph of the droplet at the entrance of the delay line, and its end. The change in the droplet projected area is noticeable without any difficulties. Furthermore, differences in the morphology of the channel contour are visible.

In figure A.1 the evolution of the droplet projected area along the delay line is presented, for a series of experiments involving the production of droplets in a $40 \mu \mathrm{m}$ deep channel. Each experiment is performed with a different surfactant concentration in the oil phase. The 
change in droplet size can be estimated to be around 30\%. In absolute value it corresponds to a change of roughly $15 \mu \mathrm{m}$, which is here too large to be explained by the enlargement of the lubrication layer following the decrease in surface tension. For a square channel, we derived in the first part the relation between the pressure drop, the channel geometry, and the flow properties, refer to the equation of a Poiseuille flow in a squared channel 4.11 derived in chapter 4. We made the following numerical application.

$$
\Delta P=\frac{Q \eta L}{C H^{3} W}
$$

with the flow rate $Q=10^{-9} \mathrm{~m}^{3} \cdot \mathrm{s}^{-1}$, the continuous fluid viscosity $\eta=1.24 \times 10^{-3} \mathrm{~Pa} \cdot \mathrm{s}$, the length of the delay line $L=121 \mathrm{~mm}$, the width of the channel (averaged) $W=200 \mu \mathrm{m}$, and the channel height $H=40 \mu \mathrm{m}$, and we take an average values of the friction coefficient $C=0.06$.

The resulting pressure drop is $\Delta P=200 \mathrm{kPa}$. In comparison the Young modulus of the PDMS is found to be of the order $Y_{\mathrm{PDMS}} \sim 600 \mathrm{kPa}$ The relative deformation $\epsilon$ of a material undergoing a pressure $P$ is:

$$
\epsilon=\frac{P}{Y}
$$

which takes the value $\epsilon=0.3$ for the pressure difference between the beginning and the end of the delay line. This value is in accordance with the changes measured in our example.

\section{A.2 Confocal Fluorescence microscopy}

The confocal microscopy has been developed by Minsky in the 1950's. The idea is based on the imaging of a focal plane with a precise field depth with the help of a pinhole. From there the imaging of a three dimensional object is reconstructed from a stack of images scanning the whole depth of the object. In the particular case of fluorescent confocal microscopy, laser light is used additionally to excite a fluorescent dye. The light emitted from the sample is then separated from the other radiations with a filter. In our case we used a solution of Resorufin $115 \mu \mathrm{mol} \cdot \mathrm{L}^{-1}$ excited with a laser light of $543 \mathrm{~nm}$. The light emitted from the sample is lead trough an Acousto Optical Beam Splitter (AOBS) where the source wavelength is filtered out. The emitted light beam is cut by the dynamic pinhole in order to select the light from the focal plane of interest. Before detection by a photomultiplier (PMT), the region of the spectrum corresponding to the emission spectrum is selected as $565 \mathrm{~nm}$ to $680 \mathrm{~nm}$. The whole setup is 


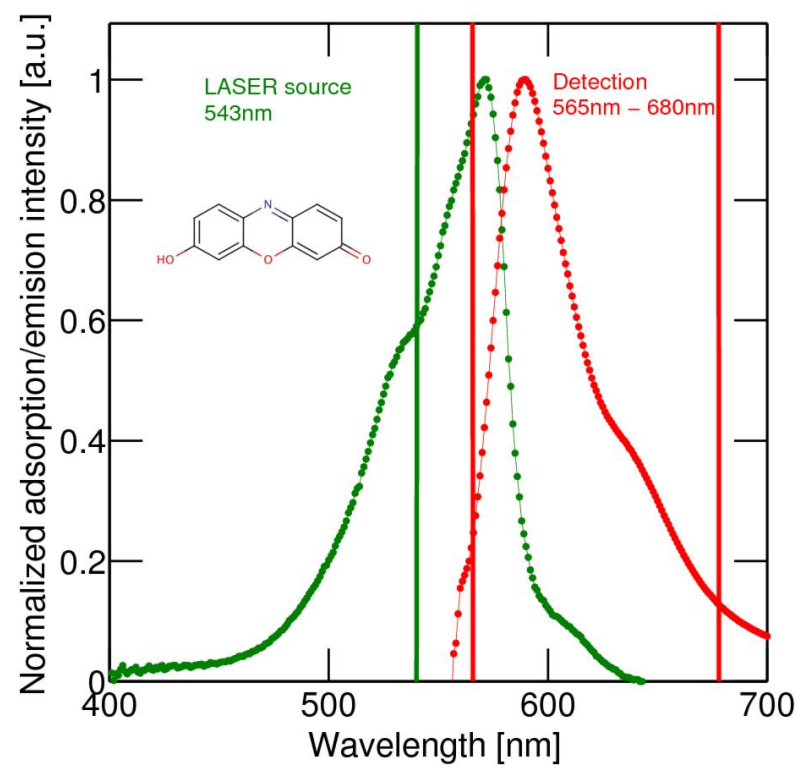

Figure A.2 Adsorption and Emission spectra for the Resorufin dye. The dye molecule is presented in the inset.

shown in figure A.3 (a).
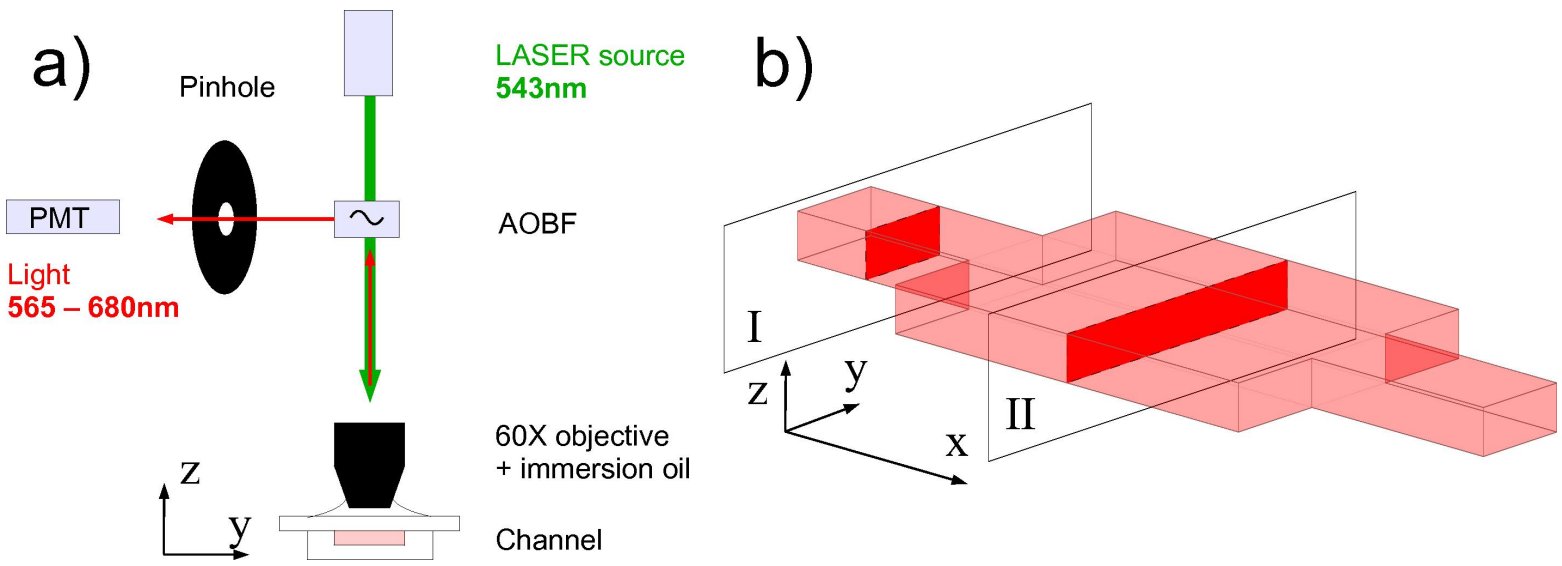

Figure A.3 (a) Schematic of the confocal fluorescence microscopy used for the determination of the channel height. (b) Detail of the planes investigated under confocal microscopy. I is situated in the channel linking two chambers. II is in the middle of the chamber.

We scan the last expansion of our channel in two different sections I and II. See figure A.3. and compare the thickness of the channel in absence of flow, $\dot{Q}=0 \mu \mathrm{L} \cdot \mathrm{min}^{-1}$, typical flow used in our experiment of droplet deformations, $\dot{Q}=60 \mu \mathrm{L} \cdot \mathrm{min}^{-1}$. For this measurement a PDMS chip with a channel height $H=45 \mu \mathrm{m}$ is bounded to a thin glass plate of thickness $e=130 \mu \mathrm{m}$, to a carry out a visualization with a X60 objective working under immersion oil in 
the near region. The resulting resolution, gives a conversion factor: 1 pixel equals $0.244 \mu \mathrm{m}$. We used the following protocol. First the channel is slowly filled with the fluorescent dye solution, in order to remove all the air bubbles potentially present. Then the flow rate is slowly brought up to $\dot{Q}=60 \mu \mathrm{L} \cdot \mathrm{min}^{-1}$ and the in flow measurement is performed. The flow rate is stopped, and equilibrated for some minutes. The measurement at rest conditions is then performed. The operation is repeated at different sections of the channel.

The final data consists of a reconstructed section of the channel in the zy-plane. Two of these planes (I) and (II) are presented on figure A.4. The light intensity is measured along a segment cutting each plane in its middle, along the z-direction. The corresponding graph allows the distinction between the glass and PDMS region, without fluorescent emission, and the inside of the channel, where light is emitted.

The intensity profile reveals a sharp transition from the glass region to the inside of the channel. We use this point as a reference for the measurement of the thickness. The interface with PDMS is less precisely defined which could be due to the migration of died solution in the porous PDMS. As predicted by the values of glass Young modulus $Y_{\text {glass }}=69 \mathrm{GPa}$ the difference in the intensity profile shows that the glass slide although very thin undergoes no deformation. We measure a channel height $H=47 \pm 2 \mu \mathrm{m}$ and $H=46 \pm 4 \mu \mathrm{m}$ for respectively planes I and II in absence of flow. These measurements are consistent with the channel height expected. In presence of flow the measured height are $H=67 \pm 2 \mu \mathrm{m}$ and $H=60 \pm 2 \mu \mathrm{m}$ respectively for planes I and II. The average deformation is then of $H=18 \mu \mathrm{m}$, which correspond to an increase of the channel height of roughly $40 \%$. 


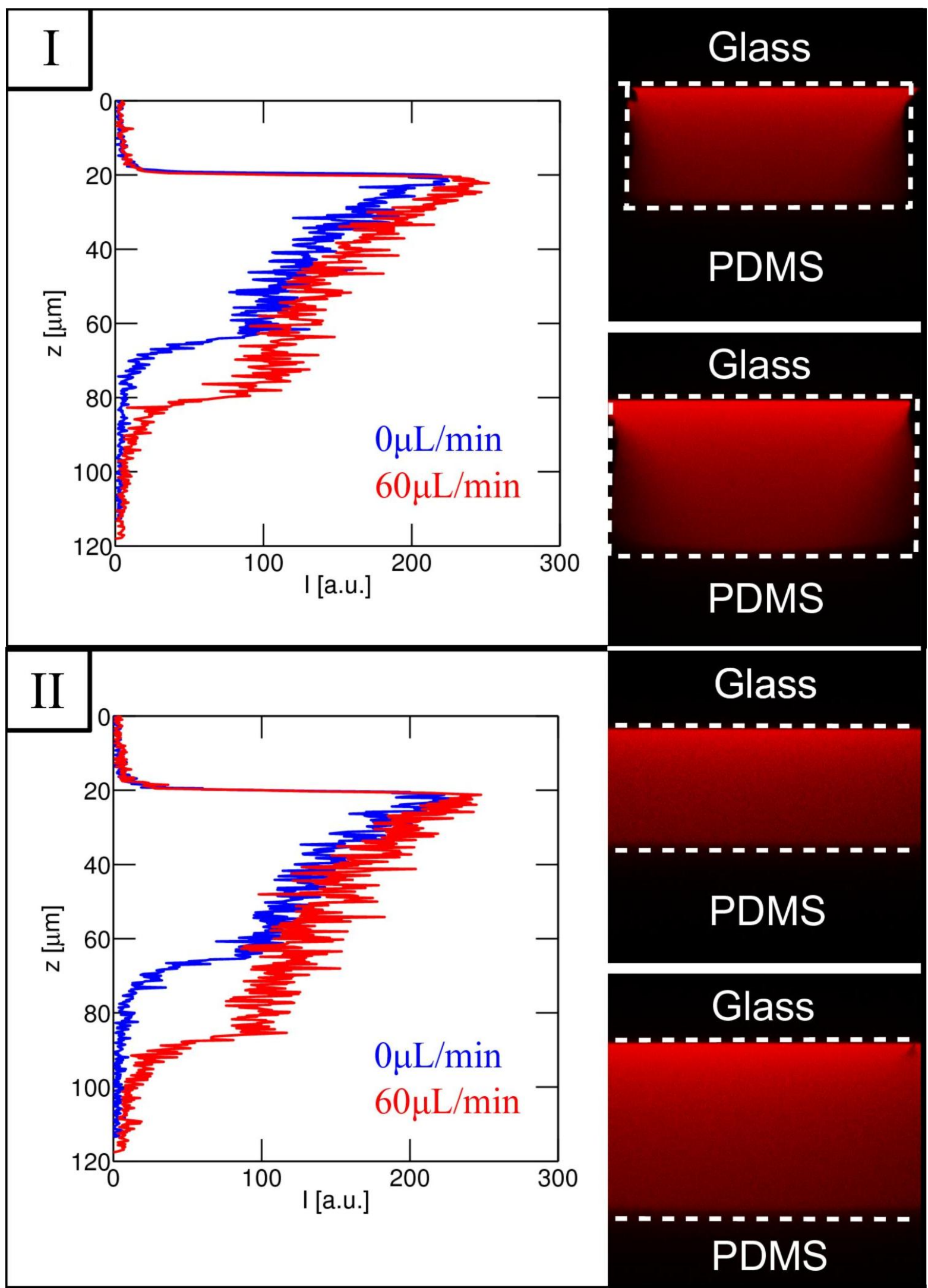

Figure A.4 Confocal fluorescence microscopy applied to the thickness measurement of a PDMS channel. The two planes I and II are investigated in condition of no flow, and for a flow rate of $\dot{Q}=60 \mu \mathrm{L} \cdot \min ^{-1}$. The graph represents the intensity measured along $\mathrm{z}$-axis in the middle of the plane I and II. 


\section{References}

\section{Bibliography}

[1] M. K. H.J. Butt, K. Graf, Physics and chemistry of interface. Wiley-VCH, 2003.

[2] S. Thutupalli, J.-B. Fleury, A. Steinberger, S. Herminghaus, and R. Seemann, "Why can artificial membranes be fabricated so rapidly in microfluidics?," Chem. Commun., vol. 49, pp. 1443-1445, 2013.

[3] M. T. Guo, A. Rotem, J. A. Heyman, and D. A. Weitz, "Droplet microfluidics for highthroughput biological assays," Lab Chip, vol. 12, pp. 2146-2155, 2012.

[4] A. Colin, T. Squires, and L. Bocquet, "Soft matter principles of microfluidics," Soft matter, vol. 8, no. 41, pp. 10527-10529, 2012.

[5] F. Franks, Water a comprehensive treatises, vol. 5. Plenum Press, 1975.

[6] S. Ip and J. Toguri, "The equivalency of surface tension, energy and surface free energy surface," Journal of material science, vol. 29, pp. 688-692, 1994.

[7] Y. Levin, "Thermodynamics of surface tension: Application to electrolyte solutions," Journal of Statistical Physics, vol. 110, no. 3-6, 2003.

[8] L. P. E. Guyon, J.P. Hulin, Hydrodynamique Physique. CNRS Editions, 2012.

[9] P. de Gennes, F. Brochard-Wyart, and D. Quéré, Gouttes, bulles, perles et ondes. Belin, 2002.

[10] T. Young, "An essay on the cohesion of fluid," Philosophical Transactions of the Royal Society of London, vol. 95, pp. 65-87, 1805. 
[11] D. Bonn, "Wetting and spreading," Review of Modern physics, vol. 81, 2009.

[12] A. Javadi, N. Moradi, H. M. ohwald, and R. Miller, "Adsorption of alkanes from the vapour phase on water drops measured by drop profile analysis tensiometry," Soft Matter, vol. 6, pp. 4710-4714, 2010.

[13] E. Franses, O. Basaran, and C. Chang, "Techniques to measure dynamic surface tension," Current opinion in colloid and interface science, vol. 1, no. 2, pp. 296-303, 1996.

[14] J. Eastoe and J. S. Dalton, "Dynamic surface tension and adsorption mechanisms of surfactants at the air-water interface.," Adv Colloid Interface Sci, vol. 85, pp. 103-144, Mar 2000.

[15] L. L. R. Miller, Progress in colloids and interface Science, vol. 2. Brill, 2011.

[16] I. G. G.T. Barnes, Interfacial Science. Oxford university press, 2011.

[17] O. Carrier, De la formation de gouttelettes à l'émulsification: approche a l'échelle experimentale. $\mathrm{PhD}$ thesis, université de Lorraine, 2012.

[18] P. Tabeling, Introduction à la microfluidique. Belin, 2003.

[19] J. Beaumont, N. Louvet, T. Divoux, M.-A. Fardin, H. Bodiguel, S. Lerouge, S. Manneville, and A. Colin, "Turbulent flows in highly elastic wormlike micelles," Soft matter, vol. 9, pp. 735-749, 2013.

[20] P. K. Vinson, J. R. Bellare, H. T. Davis, W. G. Miller, and L. E. Scriven, “Direct imaging of surfactant micelles, vesicles, discs, and ripple phase structures by cryo-transmission electron microscopy," Journal of Colloid and Interface Science, vol. 142, pp. 74-91, 1991.

[21] J. Israelachvili, Intermolecular and Surface forces. Academic press London, 1991.

[22] I. Langmuir, "A theory of adsorption," Proceedings of the American physical society, vol. 2, pp. 79-80, 1915.

[23] I. Langmuir, "The adsorption of gases on plane surface of glass, mica and platinum," Proceedings of the American physical society, pp. 1361-1402, 1918. 
[24] H. Song, D. L. Chen, and R. F. Ismagilov, "Reactions in droplets in microfluidic channels," Angew. Chem., Int. Ed., vol. 45, pp. 7336-7356, Nov 2006.

[25] B. Li, G. Geeraerts, and P. Joos, "Kinetic equation for transfer controlled adsorption kinetic," Colloids and Surfaces A: Physicochemical and engineering aspects, vol. 88, pp. 251-266, 1994.

[26] A. Bonfillon, F. Sicoli, and D. Langevin, "Dynamic surface tension of ionic surfactant solutions," Journal of colloids and interface science, vol. 168, pp. 497-504, 1994.

[27] B. Noskov, "Kinetics of adsorption from micellar solutions," Advances in Colloid and Interface Science, vol. 95, pp. 237-293, 2002.

[28] B. von Szyskowski Z. Phys. Chem., vol. 64, p. 385, 1908.

[29] A. Ward and L. Tordai, "Time dependence of boundary tensions of solutions i. the role of diffusion in time effects," Journal of chemical physics, vol. 14, p. 453, 1946.

[30] V. Fainerman, A. Makievski, and R. Miller, "The analysis of dynamic surface tension of sodium alkyl sulphate solutions, based on asymptotic equations of adsorption kinetic theory," Colloids and Surfaces A:, vol. 87, pp. 61-75, 1994.

[31] N. J. Alvarez, L. M. Walker, and S. L. Anna, "A microtensiometer to probe the effect of radius of curvature on surfactant transport to a spherical interface.," Langmuir, vol. 26, pp. 13310-13319, Aug 2010.

[32] P. Joos and G. Serrien, "Adsorption kinetics of lower alkanols at the air / water interface: Effect of structure makers and structure breakers," Journal of Colloid and lnterface Science, vol. 127, pp. 97-103, 1989.

[33] Q. Song and M. Yuan, "Visualization of an adsorption model for surfactant transport from micelle solutions to a clean air/water interface using fluorescence microscopy," Journal of Colloid and Interface Science, vol. 357, pp. 179-188, 2011.

[34] D. M. Colegate and C. D. Bain, "Adsorption kinetics in micellar solutions of nonionic surfactants.," Phys. Rev. Lett., vol. 95, p. 198302, Nov 2005.

[35] S. Köster, J. Leach, B. Struth, T. Pfohl, and J. Wong, "Visualization of flow-aligned type i collagen self-assembly in tunable ph gradients," Langmuir, vol. 23, no. 2, pp. 357-359, 2007. 
[36] A. B. Theberge, F. Courtois, Y. Schaerli, M. Fischlechner, C. Abell, F. Hollfelder, and W. T. S. Huck, "Microdroplets in microfluidics: An evolving platform for discoveries in chemistry and biology.," Angew. Chem., Int. Ed., vol. 49, pp. 5846-5868, Jun 2010.

[37] A. Lenshof, C. Magnusson, and T. Laurell, "Acoustofluidics 8: Applications of acoustophoresis in continuous flow microsystems," Lan on chip, vol. 12, pp. 1210$1223,2012$.

[38] A. Sengupta, Nematic Liquid Crystals and Nematic Colloids in Microfluidic Environment. PhD thesis, Göttingen University, 2012.

[39] J. Clausell-Tormos, D. Lieber, J.-C. Baret, A. El-Harrak, O. J. Miller, L. Frenz, J. Blouwolff, K. J. Humphry, S. K. andHoney Duan, C. Holtze, D. A. Weitz, A. D. Griffiths, and C. A. Merten, "Droplet-based microfluidic platforms for the encapsulation and screening of mammalian cells and multicellular organisms," Chemistry and Biology, vol. 15, pp. 427-437, 2008.

[40] P. Garstecki, M. J. Fuerstman, H. A. Stone, and G. M. Whitesides, "Formation of droplets and bubbles in a microfluidic t-junction scaling and mechanism of break-up," Lab on a chip, vol. 6, pp. 437-446, 2006.

[41] C. N. Baroud, F. Gallaire, and R. Dangla, "Dynamics of microfluidic droplets.," Lab Chip, Jun 2010.

[42] C. Priest, S. Herminghaus, and R. Seemann, "Controlled electrocoalescence in microfluidics: Targeting a single lamella," Appl. Phys. Lett., vol. 89, no. 13, pp. 134101-3, 2006.

[43] S. L. Anna, N. Bontoux, and H. A. Stone, "Formation of dispersions using "flow focusing" in microchannels," Appl. Phys. Lett., vol. 82, pp. 364-366, Jan. 2003.

[44] S. H. Tan, B. Semin, and J.-C. Baret, "Microfluidic flow-focusing in ac electric fields," Lab on a chip, 2014.

[45] F. Maes, S. H. Tan, and J.-C. Baret, "The microfluidic jukebox," Scientific repport, 2014. Accepted february 2014.

[46] A. R. Abate, J. Thiele, and D. A. Weitz, "One-step formation of multiple emulsions in microfluidics.," Lab Chip, vol. 11, pp. 253-258, Jan 2011. 
[47] L.-Y. Chu, A. S. Utada, R. K. Shah, J.-W. Kim, and D. A. Weitz, "Controllable monodisperse multiple emulsions," Angew. Chem. Int. Ed, vol. 46, pp. 8970-8974, 2007.

[48] A. R. Abate, C.-H. Chen, J. J. Agresti, and D. A. Weitz, "Beating poisson encapsulation statistics using close-packed ordering.," Lab Chip, vol. 9, pp. 2628-2631, Sep 2009.

[49] A. R. Abate, J. Thiele, M. Weinhart, and D. A. Weitz, "Patterning microfluidic device wettability using flow confinement.," Lab Chip, vol. 10, pp. 1774-1776, Jul 2010.

[50] J. Bibette, F. Leal-Calderon, and P. Poulin, "Emulsions: basic principles," Rep. Prog. Phys., vol. 62, pp. 696-1033, 1999.

[51] N. Bremond, A. R. Thiam, and J. Bibette, "Decompressing emulsion droplets favors coalescence," Phys. Rev. Lett., vol. 100, p. 024501, Jan. 2008.

[52] N. Bremond, H. Domejean, and J. Bibette, "Propagation of drop coalescence in a twodimensional emulsion: a route towards phase inversion," Phys. Rev. Lett., p. 214502, 2011.

[53] M. Zagnoni, G. L. Lain, and J. M. Cooper, "Electrocoalescence mechanisms of microdroplets using localized electric fields in microfluidic channels.," Langmuir, vol. 26, pp. 14443-14449, Sep 2010.

[54] V. Goust, Fluorescent silica nanoparticles for multidimensional barcoding in droplets: towards high throughput sreenning in two-phase microfluidics. PhD thesis, Université de Strasbourg, 2011.

[55] B. Binks, "Particles as surfactants?similarities and differences," Current Opinion in Colloid and Interface Science, vol. 7, no. 21-41, 2002.

[56] S. Pickering, "Emulsion," Journal of the Chemical Society, Transactions, vol. 91, no. 2001-2021, 1907.

[57] A. Studart and H. C. S. D. A. Weitz, "Arrested coalescence of particle-coated droplets into nonspherical supracolloidal structures," J. Phys. Chem. B, vol. 113, no. 3914-3919, 2009. 
[58] R. Aveyard, B. P. Binks, and J. H. Clin, "Emulsions stabilised solely by colloidal particles," Advances in Colloid and Interface Science, vol. 100-102, no. 503-546, 2003.

[59] F. Durst, Fluid Mechanics: An introduction to the theory of fluid flows. Springer, 2008.

[60] O. Reynolds, "An experimental investigation of the circumstances which determine whether the motion of water shall be direct or sinuous, and of the law of resistance in parallel channels," Philosophical Transactions of the Royal Society of London, 1889.

[61] R. Miller and L. Liggieri, Progress in colloids and interface Science, vol. 1. Brill, 2009.

[62] F. Evans and H. Wennerström, The colloidal Domain. Wiley-VCH, 1999.

[63] R. Dangla, F. Gallaire, and C. N. Baroud, "Microchannel deformations due to solventinduced pdms swelling," Lan on a chip, vol. 10, no. 21, p. 2972, 2010.

[64] E. Sollier, C. Murray, P. Maoddi, and D. Di Carlo, "Rapid prototyping polymers for microfluidic devices and high pressure injections," Lab Chip, vol. 11, pp. 3752-3765, 2011.

[65] R. Dangla, S. C. Kayi, and C. N. Baroud, "Droplet microfluidics driven by gradients of confinement," PNAS, vol. 110, no. 3, pp. 853-858, 2013.

[66] A. M. Andrew, "Another efficient algorithm for convex hulls in two dimensions," Information processing letters, vol. 9, no. 5, pp. 216-219, 1979.

[67] C. H. J. Schmitz, A. C. Rowat, S. Koester, and D. A. Weitz, "Dropspots: a picoliter array in a microfluidic device.," Lab Chip, vol. 9, pp. 44-49, Jan 2009.

[68] N. Campbell, J. Reece, L. Urry, M. Cain, S. Wasserman, P. Minorsky, and R. Jackson, Biology. Pearson international edition, 2008.

[69] D. J. Holt, R. J. Payne, and C. Abell, "Synthesis of novel fluorous surfactants for microdroplet stabilisation in fluorous oil streamsl," Journal of Fluorine Chemistry, vol. 131, pp. 398-407, 2010.

[70] I. Platzman, J.-W. Janiesch, and J. P. Spatz, "Synthesis of nanostructured and biofunctionalized water-in-oil droplets as tools for homing t cellsl," Journal of the american chemical society, vol. 135, pp. 3339-3342, 2013. 
[71] Y. Skhiri, P. Gruner, S. Semin, Q. Brosseau, D. Pekin, L. Mazutis, V. Goust, F. Kleinschmidt, A. E. Harrak, J. Hutchison, E. Mayot, J. Bartolo, A. Griffiths, V. Taly, and J. Baret, "Dynamics of molecular transport by surfactants in emulsions," Soft Matter, vol. 8, pp. 10618-10627, 2012.

[72] D. J. Holt, R. J. Payne, and W. Y. C. an Chris Abell, "Fluorosurfactants for microdroplets: Interfacial tension analysis," Journal of Colloid and Interface Science, vol. 350, pp. 205-211, 2010.

[73] C. Holtze, A. C. Rowat, J. J. Agresti, J. B. Hutchison, F. E. Angile, C. H. J. Schmitz, S. Koester, H. Duan, K. J. Humphry, R. A. Scanga, J. S. Johnson, D. Pisignano, and D. A. Weitz, "Biocompatible surfactants for water-in-fluorocarbon emulsions.," Lab Chip, vol. 8, pp. 1632-1639, Oct 2008.

[74] J.-C. Baret, F. Kleinschmidt, A. E. Harrak, and A. D. Griffiths, "Kinetic aspects of emulsion stabilization by surfactants: a microfluidic analysis.," Langmuir, vol. 25, pp. 60886093, Jun 2009.

[75] S. N. Moorkanikkara and D. Blankschtein, "New methodology to determine equilibrium surfactant adsorption properties from experimental dynamic surface tension data.," Langmuir, vol. 25, pp. 6191-6202, Jun 2009.

[76] V. Doan, R. Köppe, , and P. H. Kasail, "Dimerization of carboxylic acids and salts: An ir study in perfluoropolyether media," J. Am. Chem. Soc., vol. 119, pp. 9810-9815, 1997.

[77] J.-M. Vincent, "Noncovalent associations in fluorous fluids," Journal of Fluorine Chemistry, vol. 129, pp. 903-909, 2008.

[78] P. Gruner and J. C. baret In. Prep.

[79] A. C. Legon and D. J. Mille, "Determination of properties of hydrogen-bonded dimers by rotational spectroscopy and a classfication of dimer geometries," Faraday Discuss. Chern. Soc., vol. 73, pp. 71-87, 1982.

[80] X. Y. Hua and M. J. Rosen, "Dynamic surface tension of aqueous surfactant solutions," Journal of Colloid and Interface Science, vol. 124, no. 2, pp. 652-659, 1988. 
[81] K. J. Buckler and R. D. Vaughan-Jones, "Application of a new ph-sensitive fluoroprobe (carboxy-snarf-1) for intracellular ph measurement in small, isolated cells," European Journal of Physiology, vol. 417, pp. 234-239, 1990.

[82] M. Kjellin and I. Johansson, eds., Surfactans from renewable resources. Wiley, 2010.

[83] K. Holmberg, "Natural surfactants," Current Opinion in colloid and Interface science, vol. 6, pp. 148-159, 2001.

[84] M. J. Lawrence and G. D. Rees, "Microemulsion-based media as novel drug delivery systems.," Adv Drug Deliv Rev, vol. 45, pp. 89-121, Dec 2000.

[85] L. Schramm, ed., Surfactants: Fundamentals and applications in the petroleum industry. Cambridge University Press, 2000.

[86] M. J. Rosen, Surfactants and Interfacial phenomena. John Wiley and Sons, Inc., 2004.

[87] D. Weaire and S. Hutzler, The Physics of Foams. Oxford University Press, 1999.

[88] B. Franklin, "Of the stilling of waves by means of oil (extracted from sundry letters)," Phil. Trans., vol. 64, pp. 445-460, 1774.

[89] D. Langevin, "Dynamics of surfactant layers," Current Opinion in colloid and Interface science, vol. 3, pp. 600-607, 1998.

[90] D. Georgieva, A. Cagna, and D. Langevin, "Link between surface elasticity and foam stability," Soft Matter, vol. 5, pp. 2063-2071, 2009.

[91] G. Enhoring, "Pulsating bubble technique for evaluating pulmonary surfactant," Journal of applied physiology, vol. 43, pp. 198-203, 1977.

[92] I. Langmuir, "The shapes of group molecules forming the surfaces of liquids.," Proc Natl Acad Sci U S A, vol. 3, pp. 251-257, Apr 1917.

[93] E. Lucassen-Reynders, "Surface equation of state for ionized surfactants," Journal of physical chemistry, vol. 70, p. 1777, 1966.

[94] V. B. Fainerman, S. A. Zholob, E. H. Lucassen-Reynders, and R. Miller, "Comparison of various models describing the adsorption of surfactant molecules capable of interfacial reorientation.," J Colloid Interface Sci, vol. 261, pp. 180-183, May 2003. 
[95] F. Millet, P. Perrin, M. Merlange, and J. Benattar, "Logarithmic adsorption of charged polymeric surfactants at the air-water interface," Langmuir, vol. 18, pp. 8824-8828, 2002.

[96] J. Hutchison, D. Klenerman, S. Manning-Benson, and C. Bain, "Measurements of the adsorption kinetics of a cationic surfactant in a liquid jet ellipsometry," Langmuir, vol. 15, pp. 7530-7533, 1999.

[97] F. Jin, R. Balasubramaniam, and K. Stebe, "Surfactant adsorption to spherical particles: the intrinsic length scale governing the shift from diffusion to kinetic-controlled mass transfer," The journal of adhesion, vol. 80, pp. 773-796, 2004.

[98] S. N. Moorkanikkara and D. Blankschtein, "New methodology to determine the ratelimiting adsorption kinetics mechanism from experimental dynamic surface tension data.," J Colloid Interface Sci, vol. 302, pp. 1-19, Oct 2006.

[99] X. Li, R. Shaw, G. Evans, and P. Stevenson, "A simple numerical solution to the ward and tordai equation for the adsorption of non ionic surfactants," Computers and Chemical engineering, vol. 34, pp. 146-153, 2010.

[100] S. Lin, K. McKeigue, and C. Maldarelli, "Diffusion-controlled surfactant adsorption studied by pendant drop digitization," AIChE Journal, vol. 36, p. 1785, 1990.

[101] V. Fainerman, S. Zholob, J. Petkov, and R. Miller, "C14eo8 adsorption characteristics studied by drop and bubble tensiometry," Colloids Surf., A, vol. 323, pp. 56-62, 2008.

[102] A. Makievski, G. Loglio, J. Kraegel, R. Miller, V. Fainerman, and A. Neumann, "Adsorption of protein layers at the water/air interface as studied by axisymmetric drop and bubble shape analysis," Journal of Physical chemistry B, vol. 103, pp. 9557-9561, 1999.

[103] J. K. Ferri, C. Kotsmar, and R. Miller, "From surfactant adsorption kinetics to asymmetric nanomembrane mechanics: pendant drop experiments with subphase exchange.," Adv Colloid Interface Sci, vol. 161, pp. 29-47, Dec 2010.

[104] J. D. Martin and S. D. Hudson, "Mass transfer and interfacial properties in two-phase microchannel flows," New Journal of Physics, vol. 11, p. 115005, 2009. 
[105] N. J. Alvarez, D. R. Vogus, L. M. Walker, and S. L. Anna, "Using bulk convection in a microtensiometer to approach kinetic-limited surfactant dynamics at fluid-fluid interfaces.," J Colloid Interface Sci, vol. 372, pp. 183-191, Apr 2012.

[106] A. R. Thiam, N. Bremond, and J. Bibette, "Breaking of an emulsion under an ac electric field.," Phys. Rev. Lett., vol. 102, p. 188304, May 2009.

[107] A. R. Thiam, N. Bremond, and J. Bibette, "Adhesive emulsion bilayers under an electric field: From unzipping to fusion,” Phys. Rev. Lett., vol. 107, p. 068301, 2011.

[108] N. Bremond and J. Bibette, "Exploring emulsion science with microfluidics," Soft Matter, p. DOI: 10.1039/c2sm25923k, 2012.

[109] J. T. Cabral and S. D. Hudson, "Microfluidic approach for rapid multicomponent interfacial tensiometry.," Lab Chip, vol. 6, pp. 427-436, Mar 2006.

[110] G. Taylor, "The formation of emulsions in definable fields of flow," Proc. R. Soc. London, Ser. A., vol. 146, p. 501, 1934.

[111] J. M. Rallison, "The deformation of small viscous drops and bubbles in shear flows," Annual review of fluid mechanics. Vol.16, 1984. Van Dyke, M. Wehausen, J.V. Lumley, J.L.

[112] S. L. Anna and H. C. Mayer, "Microscale tipstreaming in a microfluidic flow focusing device," Physics of fluids, vol. 18, no. 21, 2006.

[113] M. L. J. Steegmans, A. Warmerdam, K. G. P. H. Schroën, and R. M. Boom, "Dynamic interfacial tension measurements with microfluidic y-junctions.," Langmuir, vol. 25, pp. 9751-9758, Sep 2009.

[114] M. Roché, M. Aytouna, D. Bonn, and H. Kellay, "Effect of surface tension variations on the pinch-off behavior of small fluid drops in the presence of surfactants.," Phys Rev Lett, vol. 103, p. 264501, Dec 2009.

[115] J.-C. Baret, "Surfactants in droplet-based microfluidics.," Lab Chip, vol. 12, pp. 422433, Feb 2012.

[116] H. A. Stone and L. G. Leal, "The effects of surfactants on drop deformation and breakup," Journal of Fluid Mechanics, vol. 220, 1990. Times Cited: 169. 
[117] K. Erk, J. Martin, J. Schwalbe, F. Phelan, and S. Hudson, "Shear and dilational interfacial rheology of surfactant-stabilized droplets.," Journal of colloid and interface science, vol. 377, no. 1, pp. 442-9, 2012.

[118] F. P. Bretherton, “The motion of long bubbles in tubes," Journal of Fluid Mechanics, vol. 10, no. 2, pp. 166-188, 1961.

[119] B. Dai and L. Leal, “The mechanism of surfactant effects on drop coalescence," Physics of fluids, vol. 20, p. 040802, 2008.

[120] K. J. Stebe and D. Barthès-Biesel, "Marangoni effects of adsorption;desorption controlled surfactants on the leading end of an infinitely long bubble in a capillary," Journal of Fluid Mechanics, vol. 286, pp. 25-48, 31995.

[121] S. Handayani, D. T. Chiu, E. Tjitra, J. S. Kuo, D. Lampah, E. Kenangalem, L. Renia, G. Snounou, R. N. Price, N. M. Anstey, and B. Russell, "High deformability of plasmodium vivax-infected red blood cells under microfluidic conditions," Journal of Infectious Diseases, vol. 199, no. 3, pp. 445-450, 2009.

[122] G. Tomaiuolo, M. Barra, V. Preziosi, A. Cassinese, B. Rotoli, and S. Guido, "Microfluidics analysis of red blood cell membrane viscoelasticity," Lab on a Chip, vol. 11, no. 3, pp. 449-454, 2011.

[123] S. Guido and V. Preziosi, "Droplet deformation under confined poiseuille flow," Advances in Colloid and Interface Science, vol. 161, no. 1-2, pp. 89-101, 2010.

[124] G. F. Christopher and S. L. Anna, "Microfluidic methods for generating continuous droplet streams," Journal of Physics D-Applied Physics, vol. 40, no. 19, pp. R319R336, 2007.

[125] S. Guido and G. Tomaiuolo, "Microconfined flow behavior of red blood cells in vitro," Comptes Rendus Physique, vol. 10, no. 8, pp. 751-763, 2009.

[126] N. Champagne, R. Vasseur, A. Montourcy, and D. Bartolo, "Traffic jams and intermittent flows in microfluidic networks," Physical Review Letters, vol. 105, no. 4, p. 4, 2010. 
[127] J.-C. Baret, V. Taly, M. Ryckelynck, C. A. Merten, and A. D. Griffiths, "Droplets and emulsions: very high-throughput screening in biology," Med. Sci., vol. 25, no. 6-7, pp. 627-632, 2009.

[128] Y. Tian, R. Holt, and R. Apfel, "Investigation of liquid surface rheology of surfactant solutions by droplet shape oscillations: Experiments," Journal of colloid and interface science, vol. 187, no. 1, pp. 1-10, 1997.

[129] J. Martin, J. Marhefka, K. Migler, and S. Hudson, "Interfacial rheology through microfluidics," Adv. Mater, vol. 23, pp. 426-432, 2011.

[130] A. Ramachandran, K. Tsigklifis, A. Roy, and G. Leal, "The effect of interfacial slip on the dynamics of a drop in flow: Part i. stretching, relaxation, and breakup," Journal of rheology, vol. 56, no. 1, pp. 45-97, 2012.

[131] G. Taylor, "The viscosity of a fluid containing small drops of another fluid," Proceedings of the Royal Society of London. Series A, Containing papers of a mathematical and physical character, vol. 138, no. 834, pp. 41-48, 1932.

[132] J. M. Rallison, "The deformation of small viscous drops and bubbles in shear flows," Annual review of fluid mechanics. Vol.16, 1984.

[133] L. Mazutis and A. D. Griffiths, "Selective droplet coalescence using microfluidic systems," Lab on a Chip, vol. 12, no. 10, 2012.

[134] C. Pozrikids, "A practical guide to boundary element methods," 2002.

[135] E. Kadivar, S. Herminghaus, and M. Brinkmann, "Droplet sorting in a loop of flat microfluidic channels," Journal of physics. Condensed matter, vol. 25, no. 28, p. 285102, 2013.

[136] C. W. Park and G. M. Homsy, "2-phase displacement in shaw,hele cells - theory," Journal of Fluid Mechanics, vol. 139, no. FEB, pp. 291-308, 1984.

[137] T. Cubaud, "Deformation and breakup of high-viscosity droplets with symmetric microfluidic cross flows," Physical Review E, vol. 80, no. 2, p. 4, 2009.

[138] D. DiCarlo, “Inertial microfluidics,” Lab on chip, vol. 9, pp. 3038-3046, 2009. 
[139] J. W. S. Rayleigh, “On the capillary phenomena of jets," Proceedings of the Royal Society of London., vol. 29, 1889.

[140] O. A. Basaran, "Nonlinear oscillations of viscous liquid drops," Journal of Fluid Me-

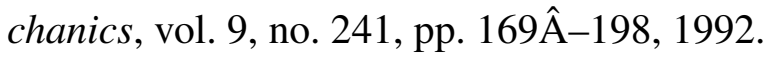

[141] J. Chrispell, R. Cortez, D. Khismatullin, and L. Fauci, "Shape oscillations of a droplet in an oldroyd-b fluid," Physica D, pp. 1593-1601, 2011.

[142] J.-C. Baret, M. M. J. DecrÃ@), and F. Mugele, "Self-excited drop oscillations in electrowetting.," Langmuir, vol. 23, pp. 5173-5179, Apr 2007.

[143] B. Ho and L. Leal, "The creeping motion of liquid drops through a circular tube of comparable diameter," Journal of Fluid Mechanics, vol. 71, no. 2, pp. 361-383, 1975.

[144] G. Hetsroni, S. Haber, and E. Wacholder, "The flow fields in and around a droplet moving axially within a tube," Journal of Fluid Mechanics, vol. 41, no. 4, pp. 689-705, 1970 .

[145] T. Mikami and S. Mason, "The capillary break-up of a binary liquid column inside a tube," The Canadian Journal of Chemical Engineering, vol. 53, pp. 372-377, 1975.

[146] J. Baret, Morphological transitions in simple eletrocapillary systems. PhD thesis, Twente University, 2005.

[147] C. D. Eggleton and K. J. Stebe, "An adsorption-desorption-controlled surfactant on a deforming droplet," Journal of Colloid and Interface Science, vol. 208, no. 1, pp. 6880, 1998.

[148] J. H. Tsui, W. Lee, S. H. Pun, J. Kim, and D.-H. Kim, "Microfluidics-assisted in vitro drug screening and carrier production," Advanced Drug Delivery Reviews, vol. 65, pp. $1575-1588,2013$.

[149] I. U. Khan, C. A. Serra, N. Anton, and T. Vandamme, "Microfluidics: A focus on improved cancer targeted drug delivery systems," Journal of Controlled Release, vol. 172, pp. 1065-1074, 2013.

[150] S. Mann, "The origins of life: Old problems, new chemistries," Angew. Chem. Int. Ed., vol. 52, pp. 155-162, 2013. 
[151] T. X. Chu, A.-V. Salsac, D. Barthès-Biesel, L. Griscom, F. Edwards-Lévy, and E. Leclerc, "Fabrication and in situ characterization of microcapsules in a microfluidic system," Microfluid Nanofluid, vol. 14, pp. 309-317, 2013.

[152] I. Polenz, S. S. Datta, and D. A. Weitz, "Controlling the morphology of polyurea microcapsules using microfluidics," Langmuir, 2014. In preparation.

[153] J. Walter, A.-V. Salsac, and D. Barthès-Biesel, "Ellipsoidal capsules in simple shear flow: prolate versus oblate initial shapes," J. Fluid Mech, vol. 676, pp. 318-347, 2011.

[154] X.-Q. Hu, B. Sévénié, A.-V. Salsac, E. Leclerc, and D. Barthès-Biesel, "Characterizing the membrane properties of capsules flowing in a square-section microfluidic channel: Effects of the membrane constitutive law," Physical Rewiew E, vol. 6, p. 063008, 2013.

[155] B. O. Shaughnessy and D. Vavylonis, "Interfacial reactions: Mixed order kinetics and segregation effects," Physical review letters, vol. 84, no. 14, pp. 3193-3196, 2000.

[156] P. de Gennes, "Polymers at an interface; a simplified view," Advances in Colloid and Interface Science, vol. 27, no. 3â€“4, pp. 189 - 209, 1987.

[157] C. Fritz, Transport de liquide et de particules dans un bord de Plateau. PhD thesis, université de Marne-la-valleé, 2006. 


\section{Acknowledgments}

Such a work can not be achieved alone. Here, I wish to acknowledge all the person that have helped me to make this work possible.

First of all, I would like to thanks Jean-Christophe Baret for giving me the opportunity of making a Ph.D. on a such interesting themes. Thanks for the close and efficient supervision along these four years. Bonne chance pour ton nouveau poste à Bordeaux. Je souhaites continuer cette active collaboration à l'avenir.

I would like also to thank all the collaborators to this thesis. First Dr. Erfan Kadivar and Dr. Martin Brinkmann, for the development and the implementation of the code used for the two dimensional simulations of the droplet deformation. Then Dr. Ingmar Polenz for the gathering the experimental data relative to the monitoring of the interfacial polymerization in microfluidics. Thanks to Dr. Anupam Sengupta and Dr. Kris Hantke for the introduction to the confocal fluorescent microscopy. Thanks to Brian Hutchinson and Estelle Mayot for the synthesis of the molecules used in this study. Finally I would like to acknowledge the contribution of all the DMI team and Dr. Benoît Semin for ideas and discussions.

Then I would like to thank Prof. Dr. Jörg Enderlein for accepting to be Referent of this $\mathrm{Ph} . \mathrm{D}$., thesis and the fruitful exchanges we had about this work. I am also very grateful to Stephan Herminghaus for discussions. Thanks also for maintaining such a high quality department at MPI DS and for being at the origin of an inspiring and productive working atmosphere.

Now, I would like to thanks all the people of Göttingen that with the time I was able to call friends. While being abroad these are the people that without knowing it, becomes your local family. Anupam, my Indian buddy and ambassador to the states, thanks for sharing your culture and tea. I hope to see you soon in New England. Ben, Danke für die grose Hilfe um Deutschland zu versthen, auch vielen danke für alle entdeckungen in die deutsche Kultur dass du hast mich gebracht. Dominika, thanks for sharing passion for good food and showing me cooking tricks from your country, Thanks for organizing this amazing trip on the Brda river. Thanks to you two and Kris for the nice Carcassonne-themed Lunches. Ciro, thanks for all the nice musical discoveries of the Romantic period. I hope to reform our duo soon, and finally nail his Brahms E-minor cello sonata. Birte thanks for bringing such a positive energy in our 
office space. Je te souhaite bonne chance en France où tu pourras profiter d'une meilleure gastronomie et des joies de la côte atlantique.

I also will not forget all the golden moments shared with Martin and Dagmar, Zrinka, Marta, Antoinette, Kris, Jeff, Elias, Eric and Paul.

Thank goes as well to Monika and to Thomas E. for the efficient administrative and technical support. Thanks for your general kindness that is bringing oil in the gears of the institute.

An enormous thank to all the people that had the patience to proof read this work: Thomas H., Julie, Zrinka, Annika, Birte, Renaud and Ciro. Without their good advices this thesis would be even less readable as it is, in the current state. "There were once two scientists...and Knudsen!".

Un grand merci à ma famille pour le support dont elle a fait preuve, bien que géographiquement très éloignée de Göttingen. Merci pour aussi avoir su garder un oeil intéressé sur mes activitées de recherche.

Thanks to Shawn Knol for giving the right to use his artwork "Visiting the meadow" and "Soap bubble 330" to illustrate the introduction of this work, see figure 1.1. I encourage all the interface fans to visit his Flickr account and be amazed:

$$
\text { www.flickr.com/photos/knol/with/9292799701/ }
$$

Finally, I will not forget J.S. Bach, J. Cage, J. Haydn, G.F. Haendel, E. Rautavaara, G. Rossini, C. Rütti and R. Vaughan-Williams for keeping me up during the witting of this work. 


\section{Curriculum Vitae}

\section{Personal Details}

Name : $\quad$ Quentin Brosseau

Date of birth : $\quad 27.02 .1986$

Place of birth : $\quad$ Saint-Nazaire, France

Address : $\quad$ Prinzenstrasse 14, 37073 Göttingen

Nationality : $\quad$ French

\section{Education}

04/2010 - 03/2014 PhD thesis at the Max Planck Institute for Dynamics \& Selforganization - Group Droplet Membrane \& Interface (Göttingen, Germany)

02/2009-09/2009 Master thesis at the University of Twente (The Netherlands) - Group Mesoscale Chemical Systems (Enschede , The Netherlands)

$2008-2009$

- Supervisor: Prof. Han Gardeniers

$2^{\text {nd }}$ Year Master of Physics: Specialisation in Nanotechnologies Toulouse University (Toulouse, France)

$2007-2008$ $1^{\text {st }}$ Year Master in Physics, Lund University (Lund, Sweden)

$2004-2007$

Bachelor in Physics Nantes University (Nantes, France) 


\section{List of Publications}

- Dynamics of molecular transport by surfactants in emulsions

Yousr Skhiri, Philipp Gruner, Benoit Semin, Quentin Brosseau, Deniz Pekin, Linas Mazutis, Victoire Goust, Felix Kleinschmidt, Abdeslam El Harrak, J. Brian Hutchison, Estelle Mayot, Jean-François Bartolo, Andrew D. Griffiths, Valérie Taly and Jean-Christophe Baret Soft Matter 8 (2012) 10618-10627

- Microfluidic Dynamic Interfacial Tensiometry ( $\mu$ DIT)

Quentin Brosseau, Jérémy Vrignon and Jean-Christophe Baret Soft Matter 2014 


\section{Erklärung}

Hiermit erkläre ich, dass ich die vorliegende Arbeit selbstständig angefertigt, nicht anderweitig zu Prüfungszwecken vorgelegt und keine anderen als die angegebenen Hilfsmittel verwendet habe. Sämtliche wissentlich verwendete Textausschnitte, Zitate oder Inhalte anderer Verfasser wurden ausdrücklich als solche gekennzeichnet.

Göttingen, im März 2014

Quentin Brosseau 